INSPECTION AND MAINTENANCE OPTIMISATION OF MULTICOMPONENT SYSTEMS

by

\author{
Vladimir Oleg Babishin \\ Master of Engineering in Mechanical and Industrial Engineering \\ Ryerson University, 2013 \\ Bachelor of Applied Science in Industrial Engineering, Minor in Bioengineering \\ University of Toronto, 2012
}

A dissertation
presented to Ryerson University
in partial fulfillment of the
requirements for the degree of
Doctor of Philosophy
in the Program of
Mechanical and Industrial Engineering.

Toronto, Ontario, Canada, 2017

(C) Vladimir Babishin, 2017 


\section{AUTHOR'S DECLARATION FOR ELECTRONIC SUBMISSION OF A DISSERTATION}

I hereby declare that I am the sole author of this dissertation. This is a true copy of the dissertation, including any required final revisions, as accepted by my examiners.

I authorize Ryerson University to lend this dissertation to other institutions or individuals for the purpose of scholarly research.

I further authorize Ryerson University to reproduce this dissertation by photocopying or by other means, in total or in part, at the request of other institutions or individuals for the purpose of scholarly research.

I understand that my dissertation may be made electronically available to the public. 


\title{
Inspection and Maintenance Optimisation of Multicomponent Systems
}

\author{
Vladimir Oleg Babishin \\ Doctor of Philosophy \\ Department of Mechanical and Industrial Engineering \\ Ryerson University \\ 2017
}

\begin{abstract}
The present research proposes methodology and mathematical models for optimisation of inspection and maintenance in complex multicomponent systems with finite planning horizon. Components are classified by failure types: hard-type and soft-type. The systems analysed are composed of either multiple identical hidden soft-type components in $k$-out-of- $n$ redundant configuration, or a combination of hard-type and hidden soft-type components. Failures of hardtype components cause system failures. Failures of components in $k$-out-of- $n$ systems and softtype component failures are hidden and not discoverable until an inspection, but reduce the system's reliability and performance. The systems are inspected either periodically, or nonperiodically. They are also inspected opportunistically at the times of system failure (occurring at $(k-n+1)^{\mathrm{st}}$ component failures in $k$-out-of- $n$ systems, or at hard failures in the systems composed of hard-type and soft-type components). Inspections have negligible duration. All components may undergo minimal repair, or corrective replacement, with hard-type components also having a possibility of preventive replacement under periodic inspections. We only consider minimal repair and corrective replacement under non-periodic inspections.
\end{abstract}


We propose several models for joint optimisation of inspection and maintenance policies that result in the lowest total expected cost. Since soft failures are hidden, we generate expected values for the number of minimal repairs, number of replacements and downtime recursively. Due to multiple component interactions and system complexity, Monte Carlo simulation and genetic algorithms (GA) are used for optimisation. Using GA for optimisation allows to consider quasicontinuous inspection intervals due to improved computational efficiency compared to Monte Carlo simulation. Some of proposed models feature preventive component replacements and are applicable even for systems with hidden component failures.

For $k$-out-of- $n$ systems, we apply periodic model to series and parallel systems and compare the results. We provide expressions for expected number of system failures in terms of cost ratio and component failure intensity. We also provide a simplified expression for system reliability. In addition, we derive a formula for finding the planning horizon length based on expected number of system failures. It may be useful for planning the system's operating horizon, at the system design stage and when analysing its performance. 


\section{Acknowledgements}

Above all, praise be to God Almighty for His unutterable mercy, abounding grace, gift of peace, true meaning, unending help and constant support! Everything that I have, or ever will accomplish is His gift of Love and is due to Him. Glory be to God and Lord Jesus Christ!

The author would like to thank Dr. Sharareh Taghipour for her guidance, advice, support and assistance. Dr. Taghipour's enthusiasm, patience, understanding and willingness to help have been invaluable and are greatly appreciated in the course and context of this work.

The author would also like to thank Dr. Mohamad Y. Jaber for providing the opportunity to feel the pleasure and satisfaction of teaching, for the interesting and encouraging discussion of learning curves, and for his support.

Many thanks go to Dr. Cory Searcy for posing questions and providing constructive feedback on the improvement of the Research Proposal. Thanks to Dr. Om Yadav from Industrial and Manufacturing Engineering Department of North Dakota State University, Dr. Arnold Yuan, Dr. Mohamad Y. Jaber, Dr. Cory Searcy and Dr. Ahmad Ghasempoor for serving on the examination committee.

Most importantly, the author would like to thank his family and friends for all their love, support, understanding and encouragement. 
ALL THINGS WERE MADE THROUGH HIM, AND WITHOUT HIM NOTHING WAS MADE THAT WAS MADE.

(John 1:3, Holy Bible). 


\section{Table of Contents}

\section{ABSTRACT}

iii

ACKNOWLEDGEMENTS v

TABLE OF CONTENTS vii

LIST OF TABLES $\mathbf{x}$

LIST OF FIGURES $\mathbf{x i}$

LIST OF APPENDICES xii

NOMENCLATURE xiii

1.1. STATEMENT OF AUTHORSHIP

2.1. SySTEM'S LIFE CYCLE LENGTH OR PLANNING HoRIZON

2.2. TYPES OF COMPONENTS IN A SYSTEM

2.3. TYPES OF MAINTENANCE ACTION

2.4. CHOICE OF MAINTENANCE ACTION

2.5. PERIODICITY OF INSPECTION

2.6. TYPES OF INSPECTION

2.7. RESEARCH GAPS

2.8. OBJECTIVES OF THE PROPOSED RESEARCH

3. JOINT OPTIMAL MAINTENANCE AND INSPECTION FOR PERIODIC $K$-OUT-OF- $N$ SYSTEMS

3.1. INTRODUCTION AND BACKGROUND

3.2. PROBLEM DESCRIPTION

3.3. MODEL 1: CORRECTIVE REPLACEMENT OF COMPONENTS AT PERIODIC INSPECTIONS 26

3.3.1. SIMULATION MODEL

3.4. MODEL 2: PREVENTIVE REPLACEMENT OF COMPONENTS AT PERIODIC INSPECTIONS 30

3.4.1. SIMULATION MODEL PROCEDURE

3.5. NUMERICAL EXAMPLES 
3.5.1. MODEL 1: CORRECTIVE REPLACEMENT OF COMPONENTS AT PERIODIC INSPECTIONS IN A K-OUTOF-N SYSTEM

3.5.2. MODEL 2: PREVENTIVE REPLACEMENT OF COMPONENTS AT PERIODIC INSPECTIONS IN A K-OUTOF-N SYSTEM

3.6. DISCUSSION AND SENSITIVITY ANALYSIS $\quad 45$

3.7. CONCLUSION $r 52$

4. OPTIMAL MAINTENANCE POLICY FOR MULTICOMPONENT SYSTEMS WITH PERIODIC AND OPPORTUNISTIC INSPECTIONS AND PREVENTIVE REPLACEMENTS

4.1. INTRODUCTION $\quad 55$

4.2. PROBLEM DESCRIPTION $\quad 62$

4.3. MODEL 3: CORRECTIVE MAINTENANCE OF HARD-TYPE COMPONENTS AND PERIODIC AND OPPORTUNISTIC INSPECTIONS OF SOFT-TYPE COMPONENTS

4.3.1. STAGE 1. OBTAINING THE OPTIMAL REPLACEMENT AGES FOR HARD-TYPE COMPONENTS 66

4.3.2. STAGE 2. OBTAINING THE OPTIMAL NUMBER OF MINIMAL REPAIRS BEFORE REPLACEMENT FOR SOFT-TYPE COMPONENTS

4.3.3. STAGE 3. OBTAINING THE OPTIMAL INSPECTION INTERVAL FOR THE SYSTEM 70

4.4. MODEL 4: PrEVENTIVE REPLACEMENT OF HARD-TyPE COMPONENTS AT PERIODIC

\begin{tabular}{lr} 
4.5. & NUMERICAL EXAMPLE \\
\hline
\end{tabular}

4.6. SENSITIVITY ANALYSIS $\quad 82$

4.7. CONCLUSION $r 6$

\section{NON-PERIODIC INSPECTION AND MAINTENANCE OPTIMISATION OF} MULTICOMPONENT SYSTEMS

5.1. $\quad$ INTRODUCTION AND BACKGROUND $\quad 89$

5.2. PROBLEM DESCRIPTION $\quad 95$

5.3. MODEL 5: JOINT OPTIMISATION OF NON-PERIODIC INSPECTION AND CORRECTIVE MAINTENANCE OF $K$-OUT-OF- $N$ SYSTEM WITH OPPORTUNISTIC INSPECTIONS

5.4. MODEL 6: NON-PERIODIC INSPECTION AND CORRECTIVE MAINTENANCE OF HARD-TYPE AND SOFT-TYPE COMPONENTS WITH OPPORTUNISTIC INSPECTION OF SOFT-TYPE COMPONENTS

$\begin{array}{lr}\text { 5.5. SIMULATION MODEL } & 104\end{array}$

5.5.1. SIMULATION MODEL FOR K-OUT-OF-N SYSTEM (SYSTEM 1) 104

5.5.2. SIMULATION MODEL FOR A SYSTEM WITH HARD-TYPE AND SOFT-TYPE COMPONENTS (SYSTEM 2) 106

5.6. NUMERICAL EXAMPLE 107

5.6.1. MODEL 5: K-OUT-OF-N SYSTEM WITH OPPORTUNISTIC INSPECTIONS 107

5.6.2. MODEL 6: SYSTEM WITH HARD-TYPE AND SOFT-TYPE COMPONENTS AND OPPORTUNISTIC $\begin{array}{ll}\text { INSPECTIONS } & 110\end{array}$

$\begin{array}{lr}\text { 5.7. CONCLUSION } & 115\end{array}$

6. CONCLUSIONS AND FUTURE RESEARCH 116 
6.1. MAIN RESULTS AND CONTRIBUTIONS $\quad 116$

\begin{tabular}{lr} 
6.2. & FUTURE WORK \\
\hline
\end{tabular}

\begin{tabular}{lr} 
APPENDICES & 125 \\
\hline
\end{tabular}

APPENDIX A. CONVEXITY OF THE OBJECTIVE FUNCTION 125

APPENDIX B. RECURSIVE FORMULATIONS FOR THE CASE OF CORRECTIVE MAINTENANCE OF HARD-

APPENDIX C. SIMULATION FLOWCHARTS FOR THE CASE OF CORRECTIVE MAINTENANCE OF HARDTYPE COMPONENTS AND OPPORTUNISTIC INSPECTION OF SOFT-TYPE COMPONENTS 


\section{List of Tables}

Table 1: Contributions of the Present Work to the Multicomponent Systems Inspection and Maintenance Optimisation. ............................................................................... 16

Table 2: Per-Component Parameters of the Power Law Intensity Function and Costs............... 36

Table 3: Upper confidence limit ( $\alpha=90 \%$ ) on the expected number of failures for

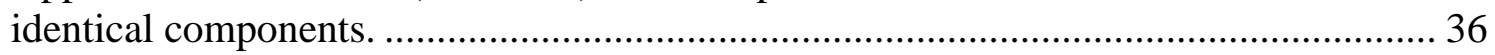

Table 4: Transposed total expected cost matrix (in $\$$ ) for different values of $m=0,1, \ldots, 5$

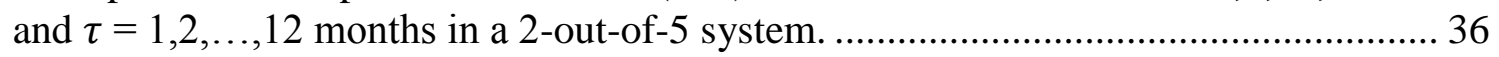

Table 5: Transposed total expected cost matrix (in $\$$ ) for different values of $m=0,1, \ldots, 5$ and $\tau=1,2, \ldots, 12$ months in a 1 -out-of-5 (parallel) system....................................... 38

Table 6: Transposed total expected cost matrix (in $\$$ ) for different values of $m=0,1, \ldots, 5$

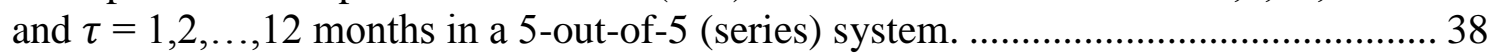

Table 7: Per-Component Parameters of the Power Law Intensity Function and Costs............... 39

Table 8: Transposed Total Expected Cost Matrix (in \$) for Different Values of $m=$ $0,1, \ldots, 5$ and $\tau=1,2, \ldots, 12$ Months in a 3-out-of-5 System.

Table 9: Ranges of system downtime penalty cost and total expected cost with the corresponding values of $\tau *$ and $F(\omega, \boldsymbol{t}, k, n)$ for $m *=5$ and other parameters as in Table 2

Table 10: Total expected costs for Model 1 and Model 2 for each inspection period $\tau=$ $1,2, \ldots, 12$ months.

Table 11: Parameters of the power law intensity function and costs for different

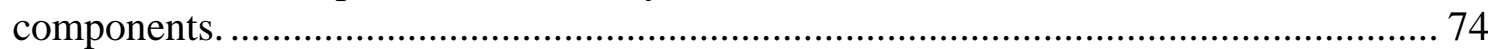

Table 12: Optimal replacement ages for hard-type components. .............................................. 75

Table 13: Confidence interval $(90 \%)$ on the expected number of failures. ............................... 75

Table 14: The optimal number of minimal repairs before replacement and the corresponding minimal expected cost for each soft-type component $s=1,2, \ldots, 5$ for inspection intervals of $\tau=1,2, \ldots, 12$ months............................................. 76

Table 15: Total expected costs for Model 3 and Model 4 for each inspection period $\tau=$ $1,2, \ldots, 12$ months.

Table 16: Distribution parameters for hard-type components at baseline and high failure

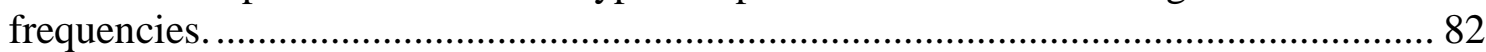

Table 17: Parameters of the Power Law Intensity Function and Costs. ............................... 107

Table 18: Optimal Policies from the Genetic Algorithm..................................................... 109

Table 19: Parameters of the power law intensity function and costs for different components of Case 1 (Baseline) and Case 2 ...................................................... 111

Table 20: Optimal maintenance policies for Case 1 and Case 2 ........................................... 113

Table 21: Optimal policies from the genetic algorithm..................................................... 113

Table 22: Absolute and relative changes in the expected costs for Case 2 compared to

Case 1. 


\section{List of Figures}

Figure 1: Surface Fitted to the Total Expected Cost Matrix for a 2-out-of-5 System.

Figure 2: Continuous graphical representation of total expected cost matrix for a 3-out-of-5

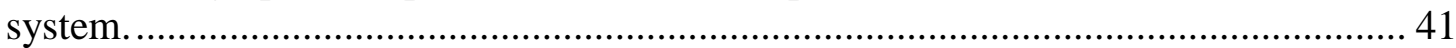

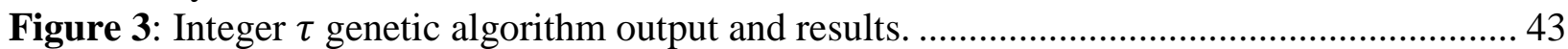

Figure 4: Quasi-continuous $\tau$ genetic algorithm output and results. ...................................... 44

Figure 5: Expected total cost vs. system downtime penalty cost for $m^{*}=5$ and the other parameters as in Table 7....................................................................................... 46

Figure 6: Range of system downtime penalty cost for which expected total cost remains optimal with $m^{*}=5, \tau^{*}=2$ and all other parameters as in Table 7 .

Figure 7: Sample soft and hard failures with periodic (scheduled) and opportunistic (unscheduled) inspections within cycle $\omega$.

Figure 8: Total expected costs for the case of baseline failure frequency of hard-type components for Model 3.

Figure 9: Total expected costs for the case of baseline failure frequency of hard-type components for Model 4.

Figure 10: Total expected costs for baseline Model 3, Model 3 with replacements occurring after LCLs number of component failures, and Model 3 with replacements occurring after UCLs number of component failures (a) and for baseline Model 4, Model 4 with replacements occurring after LCLs number of component failures, and Model 4 with replacements occurring after UCLs number of component failures (b).

Figure 11: Total expected costs for the cases of baseline and high failure frequencies of hardtype components for Model 3

Figure 12: Total expected costs for the cases of baseline and high failure frequencies of hardtype components for Model 4

Figure 13: Ranges (in \$) of the total expected cost values for baseline and high hard failure frequencies for each of Model 3 and Model 4.

Figure 14: Sample hidden failures, scheduled and opportunistic inspections within one life cycle for System 1.

Figure 15: Sample hard and hidden soft failures, scheduled and opportunistic inspections within one life cycle for System 2.

Figure 16: Genetic algorithm's output and results for Case 1 of a 3-out-of-5 system. 108

Figure 17: Genetic algorithm's output and results for Case 1 of a system composed of softand hard-type components. 


\section{List of Appendices}

Appendix A. Convexity of the objective function..... 125

Appendix B. Recursive formulations for the case of corrective maintenance of hard-type components and opportunistic inspections of soft-type components. 128

Appendix C. Simulation flowcharts for the case of corrective maintenance of hard-type components and opportunistic inspection of soft-type components 


\section{Nomenclature}

Typesetting Convention: scalars are in italics; vectors, matrices and arrays are bold.

\section{Latin Symbols}

C Cost (random variable).

E Expectation.

$F \quad$ Expected number of system failures.

$G \quad$ Generator function for the expected values used in simulations.

$I \quad$ Indicator function for the type of expected value.

$M \quad$ Expected number of minimal repairs.

$P \quad$ Probability.

$R \quad$ Expected number of replacements.

$U \quad$ Expected uptime.

$Y \quad$ Random variable for the first failure times of a soft-type component.

$Z \quad$ Random variable for the first failure times of a hard-type component.

$U C L$ Upper confidence limit.

$L C L$ Lower confidence limit.

a Inspection policy index.

$b \quad$ Random number.

c Cost (constant).

$f \quad$ Number of component failures.

$h \quad$ Hard-type component index.

$i \quad$ Scheduled inspection index.

$j \quad$ Component index.

$k \quad$ Number of identical components required to be in working order for a $k$-out-of- $n$ system.

$l \quad$ Total number of inspections within a planning horizon.

$m \quad$ Number of minimal repairs before replacement.

$n \quad$ Total number of components.

$q \quad$ Probability of hard subsystem failure.

$r \quad$ Indicator function for the choice of maintenance action.

$s \quad$ Soft-type component index.

$t \quad$ Age of a component.

$x \quad$ Inspection policy.

$y \quad$ First failure time of a soft-type component.

$z \quad$ First failure time of a hard-type component.

$\mathcal{R}$ Reliability.

\section{Greek Symbols}

$\Gamma \quad$ Gamma function.

$\Phi \quad$ Random variable for the number of component failures.

$\alpha \quad$ Confidence level.

$\beta \quad$ Shape parameter in Weibull distribution.

$\zeta \quad$ Optimal replacement age for hard-type component. 
$\eta \quad$ Scale parameter in Weibull distribution, characteristic life.

$\theta \quad$ Age of a hard-type component.

$\vartheta \quad$ Step function for the initial age of a hard-type component.

$\lambda$ Power-law intensity (hazard) function.

$\sigma \quad$ Time until next inspection.

$\tau \quad$ Minimal time unit.

$\varphi \quad$ Instance number of component failures.

$\chi \quad$ Time-to-failure.

$\psi \quad$ Placeholder function for the expected value of interest.

$\omega \quad$ Length of planning horizon (life cycle).

\section{Superscripts}

* Optimality.

T Matrix transpose.

D Component downtime.

$H \quad$ Subsystem composed of all hard-type components of a system.

I Inspection.

$M \quad$ Minimal repair.

$R \quad$ Replacement (see "corrective replacement").

$S \quad$ Subsystem composed of all soft-type components of a system.

$T$ Total.

$C R \quad$ Corrective replacement.

$H S$ System composed of hard-type and soft-type components.

$P R \quad$ Preventive replacement.

$S D \quad$ System downtime.

a Inspection policy identifier.

$k, n \quad$ System composed of $k$-out-of- $n$ components.

\section{Subscripts}

1 Reference to soft-type components.

2 Reference to hard-type components.

OI Opportunistic inspection.

c Current.

$h \quad$ Hard-type component identifier.

$i \quad$ Scheduled inspection identifier.

j Component identifier.

$l \quad$ Reference to the total number of inspections within a planning horizon.

$m$ Number of minimal repairs before replacement maintenance criterion.

$s \quad$ Soft-type component identifier.

$x \quad$ Inspection policy identifier. 


\section{Introduction}

Systems consisting of multiple parts - components - which may fail and adversely affect the functionality of the entire system, are called multicomponent systems. Such systems are encountered in a wide variety of industries, as well as in every-day life. Some of the most important factors for systems in general are their reliability and availability, since these properties most often directly influence the costs of using or operating the former. Penalties (e.g. costs of downtime and lost work) are usually incurred whenever a system's performance and/or reliability are adversely affected, e.g. a system stops operating completely (fails), or starts manifesting some sort of undesirable behaviour. For this reason, it is usually desirable to keep the system reliability and availability as high as possible. Some examples of such systems are computer network servers, backup power generation systems and medical devices. In order to make the system's reliability and availability as high as possible, while also retaining the economic feasibility, it is necessary to develop and implement optimal inspection and maintenance policies.

Mathematical optimisation models are usually developed for the purpose of generating such policies. These are based on certain assumptions that can be classified by the aspects of the system they relate to, such as:

1. System's life cycle length, or equivalently, planning horizon - time period (range) over which the system's operation is considered and its maintenance and/or inspection are optimised. The usual assumptions pertaining to this aspect are:

a. Infinite planning horizon - a very long period is assumed over which a system operates, or no real consideration is given to the planning horizon's length, since the costs are expressed per unit of time.

b. Finite planning horizon - an operating period of a particular length is chosen, over which the calculations are performed.

2. Types of components composing the system, generally classified by their corresponding types of failure. These may be subdivided into:

a. Non-identical components - components whose parameters and/or pertinent operational assumptions differ from one another. Examples of such include: 
i. Hard-type components - components which fail in a "hard failure" mode, i.e. cause the entire system to fail simultaneously with their failure. Systems composed entirely of hard-type components are identical to systems in series configuration, as failure of one component immediately causes the failure of the entire system.

ii. Soft-type components - components that fail in a "soft failure" mode, i.e. do not cause immediate failure of the system, but reduce its reliability.

iii. Hidden (hidden soft-type) components - components that fail in a "hidden failure" mode, i.e. which are part of protective, reserve, or safety equipment, whose operation is not normally required when the system they protect is in a working order. However, when the system fails, the protective equipment has to start operating, and if it is unable to perform, then its failure is revealed. Similarly to soft failures, hidden (or hidden soft) failures are not revealed until inspection occurs, but unlike soft failures, they do not affect the protected system's operation until it fails and the protective equipment has to perform.

b. Identical components - components which have identical operational assumptions and parameters. Examples of such components are encountered in various redundant systems, such as in $k$-out-of- $n$ system configurations. It can be also noted that the system is still operational under the condition that the current number of failed components in a $k$-out-of- $n$ : $G$ system remains less than or equal to $n-k$. Such systems can be regarded as composed entirely of identical soft-type components.

3. Types of maintenance action - kinds of operations performed on the failed component with the purpose of returning it back into service, i.e. to the functioning state. The following types of maintenance actions are widely used in the literature:

a. Minimal repair - action of restoring the component to the state it was in just prior to failure. In the mathematical sense, minimal repair does not affect the age of the component, i.e. the component's age is unchanged by the minimal repair and remains the same immediately after it. 
b. Replacement - action of restoring the component to the state it was originally in. Mathematically, it is expressed in the component's age's being reset to 0 .

c. Imperfect repair - action of restoring the component to the state intermediate between minimal repair and replacement. Mathematically, it is usually represented as assigning some percentage of the age at failure to the component restored using imperfect repair maintenance.

4. Criterion for choosing maintenance action - choice of how to handle components that are deemed to be in need of intervention from outside of the system (i.e. maintenance). The following criteria are used for choosing the maintenance action for a failed component:

a. Age of component - this is the most common criterion used for maintenance decision when the necessary information about the component parameters is available (e.g. hours of operation, mileage, etc.). This criterion is convenient, as it directly translates into the operational time of the component and, therefore, allows for a practical monitoring of the component's "health".

b. Number of failures - number of times a component has failed in the past. This criterion is most popular when the component's age is not known, which is usually the case for components subject to soft and hidden failures. This number gives rise to another metric - the number of minimal repairs before replacement.

c. Probability of a particular type of maintenance action - this is usually based on the age of a component, which is included as a parameter in the probability function.

5. Periodicity of inspection - frequency with which inspections are performed on the system's components. Two types of (mutually exclusive) periodicity are usually considered in the literature when considering inspection policies:

a. Periodic inspections - inspections occurring at pre-determined regular time intervals (with the possible exception of the last inspection; see 'Methodology' for more details).

b. Non-periodic inspections - inspections occurring at possibly irregular time intervals. 
6. Type of inspection - kind of inspection performed on the system's components. Most often, the following types of inspection are noted in the literature:

a. Scheduled, planned inspection - inspection prescribed by the inspection policy.

b. Opportunistic inspection - inspection not prescribed by the inspection policy, but performed as a result of the opportunity arising from a system event (e.g. system failure, which may be triggered by failure of a hard-type component).

Given such classification of the most widely-used assumptions in the literature, the focus of the present research can be defined. The aspects of the systems most relevant to the present research (and their corresponding assumptions from the list above) are: finite system life cycle and planning horizon (Assumption 1b), systems composed of both non-identical (Assumption 2a) and identical (Assumption 2b) components, subject to minimal repairs (Assumption 3a) and replacements (Assumption 3b), age-based and count-based criteria for choosing maintenance action type (Assumptions 4a and 4b), subject to periodic (Assumption 5a) and non-periodic (Assumption 5b), planned (Assumption 6a) and opportunistic (Assumption 6b) inspections.

Furthermore, it should be noted that, according to the scope of the relevant aspects of the classification scheme presented above, the focus of the present research is on the complex systems. A complex system is defined as a system displaying synergy as a result of interaction of its components, i.e. the effect of such interaction cannot be described simply by superposing the effects of individual components. Thus, the combined effect of interaction of parts or components of a complex system does not obey the properties of superposition. Contrary to the complex systems, in a simple system, the effects of component interactions can be described using superposition, i.e. the combined effect of interaction among the constituent components of a simple system is equivalent to the sum of the individual effects of each component.

The further organisation of the present dissertation is as following.

Chapter 2 contains a summary of the relevant literature.

Chapter 3 focusses on the periodic inspection and maintenance optimisation models for $k$ out-of- $n$ systems. Two models are proposed: the first assumes minimal repair or corrective replacement as the only maintenance actions; the second considers preventive replacement of components in addition to those in the first model. This chapter is based on the paper entitled "Joint Optimal Maintenance and Inspection for a $k$-out-of- $n$ System", published in the 
International Journal of Advanced Manufacturing Technology, as well as the paper entitled "Joint Maintenance and Inspection Optimization of a $k$-out-of- $n$ System", presented at the Annual Reliability and Maintainability Symposium (RAMS) 2016 and published in the Proceedings of the Annual Reliability and Maintainability Symposium 2016.

Chapter 4 deals with periodic inspection and maintenance models for complex systems composed of hard-type and hidden soft-type components. We propose two models: one assuming minimal repair or corrective replacement for the available maintenance actions; the other also considering preventive replacement of hard-type components at the periodic inspection times. This chapter is based on the paper entitled "Optimal Maintenance Policy for Multicomponent System with Periodic and Opportunistic Inspections and Preventive Replacements", published in the Applied Mathematical Modelling journal.

Chapter 5 concentrates on the non-periodic optimisation of inspection and maintenance for multicomponent systems over a finite life cycle. For the $k$-out-of- $n$ system, we find the optimal joint bivariate policy composed of the optimal number of minimal repairs before replacement and the optimal inspection scheme resulting in the minimal total expected cost. For the hardand-soft-type system, we find the optimal joint trivariate policy composed of the optimal replacement ages for the hard-type components, the optimal number of minimal repairs before replacement for the soft-type components and the optimal inspection scheme resulting in the minimal total expected cost. This chapter is based on the paper entitled "Non-Periodic Inspection and Maintenance Optimisation of Multicomponent Systems", submitted to the Computers and Industrial Engineering journal.

Chapter 6 provides a summary of major conclusions and a direction for future research.

References section compiles a list of bibliographical references used throughout this dissertation.

Appendices contains more detailed mathematical formulations and derivations that were not included in the main body of the present dissertation.

\subsection{Statement of Authorship}

Most of the chapters presented in this dissertation are the extensions of the following journal and conference papers, which are published, accepted or submitted for publication: 


\section{$\underline{\text { Peer-Reviewed Journal Articles }}$}

1. Babishin V. and Taghipour S. Joint Optimal Maintenance and Inspection for a $k$-out-of$n$ System. (2016). International Journal of Advanced Manufacturing Technology, vol. 87, no. 5, pp. 1739-1749. Published online at: http://dx.doi.org/10.1007/s00170-0168570-z. [Used in Chapter 3].

Principal author's contribution: developing the mathematical and simulation models, programming the models, running the numerical examples, discussion of the results, sensitivity analysis, and drafting the paper.

Second author's contribution: verification of the results and proofreading the draft paper.

2. Babishin V. and Taghipour S. Optimal Maintenance Policy for Multicomponent System with Periodic and Opportunistic Inspections and Preventive Replacements. (2016). Applied Mathematical Modelling, vol. 40, no. 23-24, pp. 10480-10505.

Published online at: http://dx.doi.org/10.1016/j.apm.2016.07.019. [Used in Chapter 4]. Principal author's contribution: developing the mathematical and simulation models, programming the models, running the numerical examples, discussion of the results, sensitivity analysis, and drafting the paper.

Second author's contribution: verification of the results and proofreading the draft paper.

3. Babishin V., Hajipour Y., Taghipour S. Non-Periodic Inspection and Maintenance Optimisation of Multicomponent Systems. Computers and Industrial Engineering. Under revision following reviewer's comments. [Used in Chapter 5].

Principal author's contribution: developing the mathematical and simulation models, programming the models, running the numerical examples, discussion of the results, sensitivity analysis, and drafting the paper.

Second author's contribution: programming the models.

Third author's contribution: verification of the results and proofreading the draft paper.

\section{$\underline{\text { Peer-Reviewed Conference Articles }}$}

4. Babishin V. and Taghipour S. (2016). Joint Maintenance and Inspection Optimization of a $k$-out-of- $n$ System. Proceedings of the Annual Reliability and Maintainability 
Symposium 2016, pp. 523-528. Published online at:

http://dx.doi.org/10.1109/RAMS.2016.7448039. (This paper received the American Society for Quality (ASQ)-Reliability Division (RD) Best Paper Award of the 2016 Annual Reliability and Maintainability Symposium (RAMS)). [Used in Chapter 3].

Principal author's contribution: developing the mathematical and simulation models, programming the models, running the numerical examples, discussion of the results, sensitivity analysis, and drafting the paper, preparation of the presentation, presenting at the conference.

Second author's contribution: verification of the results and proofreading the draft paper.

5. Babishin V. and Taghipour S. (2015). Maintenance and Inspection Optimization of a System with Hidden Failures. Proceedings of the IIE Annual Conference 2015, pp. 1553-1562.

Principal author's contribution: developing the mathematical and simulation models, programming the models, running the numerical examples, discussion of the results, sensitivity analysis, and drafting the paper, preparation of the presentation.

Second author's contribution: verification of the results and proofreading the draft paper, presenting at the conference. 


\section{Literature Review}

The present section is organised according to the order of assumptions stated in the 'Introduction'. Literature relevant to each of the assumptions is critically reviewed and is organised under relevant subsections.

\subsection{System's Life Cycle Length or Planning Horizon}

The majority of the available maintenance models structure the costs per unit of time, for convenience, and optimise them over an infinite planning horizon. Surveys in this regard include Wang [1], where he considers different inspection and maintenance policies for both single-unit and multicomponent systems. However, his emphasis is on systems consisting of a single component. Cho and Parlar [2] provide a survey of the models that consider infinite time horizon. None of the models in these surveys, however, considers optimisation of both inspection and maintenance over a finite time horizon.

Since the costs over infinite time horizon are not measurable, the objective function to be optimised is typically expressed in terms of the cost rate, i.e. cost per unit of time. For example, Wang and Zhang [3] propose an optimal mixed bivariate policy to minimise the average cost rate, based on a critical reliability level and the number of system failures for a simple system. Zhang and Wang [4] establish an optimal replacement policy based on the number of component failures for a series system with dissimilar components under a geometric process. They optimise their costs per unit of time.

$\mathrm{Wu}$ and Zhang [5] consider an infinite-horizon bivariate maintenance policy that is dependent on the interval length between preventive replacements and the number of component failures for a two-component cold-standby system subject to Poisson shocks. Coria et al. [6] develop an analytical optimisation method based on a new hazard function for imperfect preventive maintenance policy over an infinite planning horizon. Pan et al. [7] consider a preventive maintenance model with an improvement factor for an infinite planning horizon.

As can be seen from the references provided, none of them considers optimisation over a finite time horizon. Only recently, some work has started to appear in this direction. For 
example, Taghipour and Banjevic [8] propose models for finding the optimal inspection interval for a multicomponent system with hidden failures over both finite and infinite planning horizons. Taghipour [9], Kassaei and Taghipour [10], and Taghipour and Kassaei [11] develop models for finding the optimal inspection interval by minimising the total expected cost over a finite-time life cycle for $k$-out-of- $n$ load-sharing systems, where each failure increases the hazard rates for the remaining operational components. These models, however, do not feature maintenance and inspection optimisation.

The present dissertation is aimed at advancing the research knowledge by developing models that consider inspection and maintenance optimisation over finite time horizon for multicomponent systems.

\subsection{Types of Components in a System}

As has been previously stated, the components of a multicomponent system may be conveniently classified by type, or mode of failure into either hard, or soft [10]. With regard to purely hard-type component systems, series systems are considered, for example, by Zhang and Wang [4] and Zhou et al. [12]. Wang and Kuo [13] compare series systems with mixed (cold and warm) standby components using the mean time to failure, long-term availability and the cost/benefit ratio analysis.

Systems consisting of strictly soft-type components may be considered in the context of redundant $k$-out-of- $n$ configurations, although are rarely encountered in the literature. Instead, soft failures are considered in systems, where individual components may fail in both hard and soft failure modes. Thus, soft-type components are typically analysed in conjunction with one or more hard-type components.

Multicomponent systems with hard-type and soft-type components are considered, for example, by Taghipour and Banjevic in [8, 14, 15]. In their first model [14], hard-type components are not inspected periodically, but soft-type components are inspected periodically and opportunistically at hard failure times. In their second model [15], hard-type components are periodically inspected and preventively replaced, depending on their condition at inspection. These models were subsequently extended and modified by these and other authors. 
Systems, such as protective devices, usually contain components whose failures can be classified as hidden. Lienhardt et al. [16] define hidden failures as failures not apparent during the normal operation of a system, but only discoverable upon inspection. They provide a maintenance policy for a repairable system consisting of one component, subject to hidden failures and both corrective action and periodic inspection; they also obtain the optimal maintenance policy by minimizing the maintenance cost rate subject to the risk of corrective maintenance and provide exact analytical formulae for the case of a constant failure rate [16]. Because of the constant failure rate assumption, however, their analytical formulae are applicable only to homogeneous Poisson processes. Hidden failures for single-component systems are also investigated by Sheu et al. [17]. Systems consisting of one component and other assumptions used by Lienhardt et al. [16] and Sheu et al. [17], however, significantly limit the scope of application of their models.

Nakagawa et al. [18] obtain the optimal testing time, the expected number of tests and the expected cost until fault detection, based on the theory of Markov renewal processes for a computer system with intermittent faults. They assume a constant known probability of failure due to hidden faults and obtain a finite testing time from a solution to their equation [18]. The assumption of a known probability of failure is too restrictive for any practical purposes, since it usually demands knowledge of the previous history of failures, which itself requires observing a large number of failures in the first place to draw any statistically-meaningful results.

Bjarnason et al. $[19,20]$ consider hidden failures in the context of a $k$-out-of- $n$ redundant system and develop a joint optimisation model for minimising the total cost of both maintenance and inventory policies. Bjarnason and Taghipour [21] use genetic algorithm to search for a solution of a three-dimensional objective function to jointly find the optimal maintenance and $(\mathrm{s}, \mathrm{S})$ inventory policies for a $k$-out-of- $n$ system with hidden failures. None of these works, however, considers joint optimisation of inspection and maintenance for a $k$-out-of- $n$ system.

Based on the surveyed literature, it can be concluded that there exists a clear gap in the literature concerning the optimisation of both inspection and maintenance policies for multicomponent systems composed of hard-type and hidden soft-type components, as well as for $k$-out-of- $n$ systems with identical components. 


\subsection{Types of Maintenance Action}

The majority of researchers considers either one, or two types of maintenance action. For example, Taghipour and Banjevic [14] assume in their first model minimal repairs. Fard and Zheng [22] consider a non-repairable multicomponent system, which implies that the only type of maintenance available to them is replacement. Makis and Jardine [23] consider a system subject to replacements, which incur a smaller cost if done prior to failure than after it. Such models bear, however, a rather theoretical significance than a practical one, since they only consider one type of maintenance action. Most multicomponent systems are designed to allow for repairs. This is done because it is usually more economical to repair a system than to replace it with the new, or "as good as new" identical system [24]. For this reason, of more interest are those models that consider a variety of maintenance actions, such as both minimal repair and replacement.

Lienhardt et al. [16] and Chien and Sheu [25] consider two types of maintenance action: minimal repairs and replacements (also called "perfect repairs") for one-component systems, while Taghipour and Banjevic $[8,15]$ consider these types of maintenance for multicomponent systems, but they only optimise for periodic inspection policies. Generally, there is a lack of literature that considers both types of maintenance in the context of complex multicomponent systems and that attempts to find the optimal maintenance and inspection policies. The present thesis contributes to this research area.

\subsection{Choice of Maintenance Action}

One approach used to determine the maintenance action is presented by Sheu et al. [26], who propose a model for a single-component system with the probability of type II (catastrophic) failure that depends on the number of repairs previously performed. In their article, a maintenance decision is assumed to depend on the age at failure and the number of overhauls (similar to minimal repairs) [26]. However, although this model is a generalisation of several previous models, it considers only simple one-component systems.

Many of the surveyed models assign some probability to each type of maintenance action. Makis and Jardine [23], for example, consider minimal (imperfect) repairs for a one-component system, and in their model the probability of perfect maintenance depends on the number and 
time of imperfect maintenance actions in a cycle. Sheu and Griffith [27] consider age-dependent probabilities for the type of maintenance (replacement, or minimal (imperfect) repair). Chien and Sheu [25] assume that the probability of replacement depends on the system's age and the number of shocks tolerated by a system since last replacement. All of these models are developed for one-component systems. The scope of the present research is, however, on multicomponent systems.

Problems in multicomponent system maintenance models are surveyed by Nowakowski and Werbinka [28]. Among other policies, they consider an opportunistic (L-u, L) policy proposed by Fard and Zheng [22], in which whenever a component is replaced on failure or its failure rate reaches a certain limit $\mathrm{L}$, all other components with their failure rates falling in $(\mathrm{L}-\mathrm{u}, \mathrm{L})$ are also replaced. The method of Fard and Zheng [22] is an approximation, since the differential equation formulation they propose is generally difficult to solve due to a complicated boundary condition. The criterion they employ is related to age-based replacement, since it relies on the failure rates of the units.

With regard to multicomponent system maintenance, Zhang and $\mathrm{Wu}$ [29] consider a $k$-outof- $n$ system with perfect component repairs and imperfectly-repairable maintenance equipment (called "repair-equipment" in the article); they propose a model that minimises the expected cost rate of the repair-equipment, which is based on the optimal replacement policy for the number of repair-equipment failures. In this policy, however, only replacements of main components (perfect repairs) are considered, while minimal repairs are applied to the maintenance equipment. The present research, however, is concerned with relaxing the restrictive assumption of only one type of available maintenance for a multicomponent system.

In a second inspection optimisation model developed by Taghipour and Banjevic [14], they assume that soft-type components are either minimally repaired, or replaced with age-dependent probabilities. Instead of basing maintenance action on an age-dependent probability, a different approach is taken in the present research. Park [30] proposes the concept of the optimal number of minimal repairs before replacements where he establishes an optimal maintenance policy for one-component systems. A similar approach is adapted for multicomponent systems in the present research. It is assumed that the optimal maintenance action is directly dependent on either the age, or the number of maintenance actions (minimal repairs) previously performed, 
depending on the type of component. In addition, the choice of maintenance action is also subject to optimisation in the present research.

\subsection{Periodicity of Inspection}

Another aspect that is essential to the analysis of any inspection policy is the periodicity of the latter. A general multicomponent periodic inspection framework was proposed by Ozekici [31]. Periodic inspection policies for complex multicomponent systems have been extensively studied by Taghipour et al. [24], Taghipour and Banjevic [8, 14, 15]. Taghipour and Kassaei [11] study periodic inspection optimisation for $k$-out-of- $n$ systems.

Non-periodic inspection optimisation has also been covered in the literature. For example, Zhao et al. [32] consider a one-component system subject to replacement maintenance under Gamma deterioration process over infinite planning horizon. They develop a model similar to proportional hazards model to optimise the system's non-periodic inspection policy [32]. Su [33] essentially develops a model for optimising non-periodic inspections for a one-component multi-mode system with a combination of hidden and self-announcing operating modes, since his inspection "period" is a random variable, which renders it non-periodic according to the definitions and terminology adopted in the present dissertation. He uses the supplementary variable technique to find the optimal inspection period that maximises profit per unit time.

Multicomponent non-periodic inspections have also been considered in the literature. Castanier et al. [34, 35] propose a condition-based maintenance policy for optimal inspection and replacement of a two-component system under non-periodic inspections. They develop separate policies for each component by assuming component independence, admitting that extending their approach to larger systems makes the numerical solution intractable. In this regard, it is worth mentioning that Vaurio notes in [36] that it is not generally possible to obtain an analytical solution for the optimal inspection interval even for the simple case of optimising only for system availability. This explains the interest in and the value of numerical and simulation methods for the analysis of multicomponent systems.

Golmakani and Moakedi [37] develop a model for non-periodic inspection optimisation using dynamic programming and branch-and-bound technique. They introduce the $A^{*}$ search algorithm, which improves on the efficiency of branch-and-bound technique by branching only 
on the most promising nodes at each step of the search [37]. However, the A* algorithm is at a disadvantage for generating a large number of nodes at each iteration. Some researchers, e.g. Lapa et al. [38], demonstrated the applicability and usefulness of genetic algorithms to the optimisation of system availability. The present research also uses genetic algorithms for the purpose of improving the efficiency of optimisation calculations.

Based on a review of the literature, there appears to be a gap in the research dealing with multicomponent inspection and maintenance optimisation models for both periodic and nonperiodic inspections. There is a lack of models that would be general enough to include several types of components and maintenance actions and would at the same time have efficient implementations for practical analysis and calculations. The development of models and techniques satisfying these conditions is another goal of the current research.

\subsection{Types of Inspection}

Naturally, the innate characteristic features of any inspection policy are planned (scheduled) inspections. These inspections are generally encountered in any literature concerned with inspection or maintenance optimisation. For this reason, planned inspections are not dwelled on in the present section.

Instead, the focus in the present section is placed on opportunistic inspections, which have received an extensive coverage in the literature.

For example, Zhou et al. [12] consider opportunistic preventive maintenance optimisation for a multicomponent system with series configuration (i.e. a system consisting only of hardtype components). Owing to only one type of components, such model has limited practical applications.

In Dagpunar's model of a multicomponent system [39], if the failed component's age exceeds a specified control limit, it is opportunistically replaced. He, however, makes no distinction between the types of components, and a maintenance decision is based on the component's age rather than its maintenance history.

Zhu et al. [40] propose an opportunistic maintenance policy for multicomponent system with hard-type and soft-type components, for the case of maintaining offshore wind turbines. In 
their model, however, maintenance action for soft-type components is again dependent on the ages of the latter.

Cui and $\mathrm{Li}$ [41] consider a multicomponent system with opportunistic maintenance and stochastically-dependent components and use a cumulative damage shock model to stochastically compare maintenance policies. However, all of the components in their model are assumed to fail when exceeding some predefined threshold, and the authors assume only opportunistic maintenance [41].

Preventive replacement opportunities following a Poisson process for block and age-based replacement models are considered by Aven and Dekker [42]. Taghipour and Banjevic [14] consider minimal repairs of hard-type and soft-type components over a finite planning horizon with several opportunistic inspections in one of their models. In another paper, Taghipour and Banjevic [43] consider optimal inspection policy for complex multicomponent systems with hard and hidden soft failures under both periodic and opportunistic inspections and minimal repair or replacement maintenance options. In these models, however, the component's maintenance action is determined by the age-dependent probabilities and is not subject to optimisation.

Opportunistic inspections for $k$-out-of- $n$ systems have also received some coverage in the literature. Huynh et al. [44] provide an opportunistic condition-based maintenance model for maintenance optimisation at the component level. Huynh et al. [45] also propose a multi-level decision-making model. These, however, do not cover the optimisation of inspection frequency.

\subsection{Research Gaps}

Based on the preceding discussion, it can be seen that there are gaps in the literature related

to determining the optimal maintenance action to be taken when a component fails in a multicomponent system under finite planning horizon, periodic or non-periodic, scheduled and opportunistic inspections, where components are subject to minimal repairs and preventive or corrective replacements. A typical analysis involves the optimisation of either inspection, or maintenance policies, but not both. With optimisation of inspection policies, many authors tend to use rather strong assumptions on the type of maintenance policy to be taken, e.g. its 
dependence on the age. Although not an unreasonable assumption, it limits the scope and the practical application of such models. The proposed research is aimed at addressing these gaps.

Relevant papers from the literature are summarised and compared in Table 1, where checkmarks in the relevant columns denote certain aspects of multicomponent systems.

Table 1: Contributions of the Present Work to the Multicomponent Systems Inspection and Maintenance Optimisation.

\begin{tabular}{|c|c|c|c|c|c|c|c|c|c|c|c|c|c|}
\hline $\begin{array}{r}\text { Area of } \\
\text { Research }\end{array}$ & 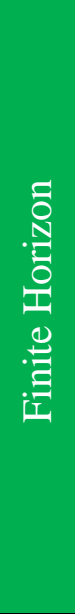 & 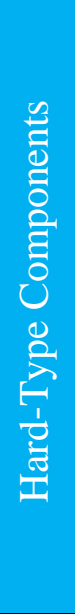 & 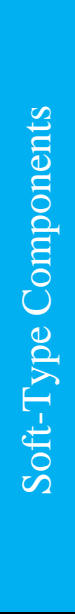 & 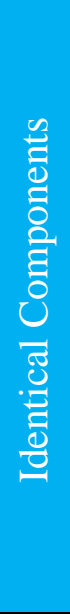 & 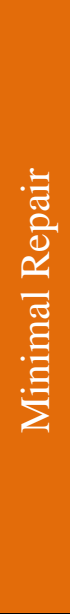 & 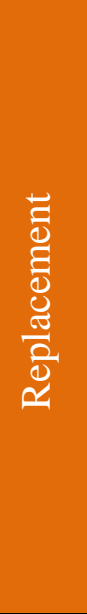 & 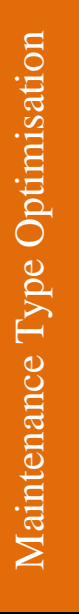 & 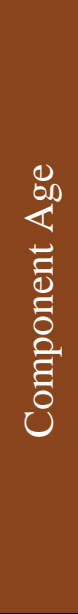 & 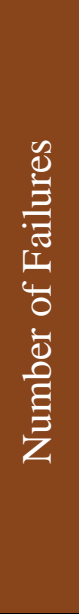 & $\begin{array}{l}0 \\
0 \\
0 \\
0 \\
0 \\
0 \\
0 \\
0 \\
0 \\
0 \\
0 \\
0 \\
0\end{array}$ & $\begin{array}{l}0 \\
0 \\
0 \\
0 \\
0 \\
0 \\
0 \\
0 \\
0 \\
0 \\
0 \\
0 \\
0 \\
0 \\
0 \\
0\end{array}$ & 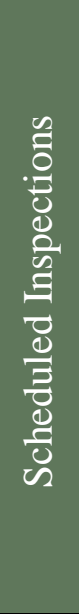 & 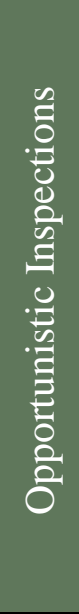 \\
\hline $\begin{array}{c}\text { Aven \& } \\
\text { Dekker } \\
\text { (1997) }\end{array}$ & $\checkmark$ & & & & $\checkmark$ & $V$ & & $\checkmark$ & & & & $\checkmark$ & $\checkmark$ \\
\hline $\begin{array}{l}\text { Anisimov \& } \\
\text { Gürler } \\
(2003)\end{array}$ & $\checkmark$ & & & $\checkmark$ & & $V$ & & & & & & & \\
\hline $\begin{array}{c}\text { Wang \& } \\
\text { Christer } \\
\text { (2003) }\end{array}$ & & & & & & $\checkmark$ & & $\checkmark$ & & & & $\checkmark$ & $\checkmark$ \\
\hline $\begin{array}{c}\text { Castanier et } \\
\text { al. } \\
(2005)\end{array}$ & & & & & & $\checkmark$ & & & & & $\checkmark$ & & $\checkmark$ \\
\hline $\begin{array}{l}\text { Nakagawa } \\
\& \text { Mizutani } \\
(2007)\end{array}$ & $\checkmark$ & & & & $\checkmark$ & $\checkmark$ & & & & $\checkmark$ & & & \\
\hline $\begin{array}{c}\text { Hartman \& } \\
\text { Murphy } \\
\text { (2006) }\end{array}$ & $\checkmark$ & & & & & $V$ & & $\checkmark$ & & & & & \\
\hline
\end{tabular}




\begin{tabular}{|c|c|c|c|c|c|c|c|c|c|c|c|c|c|}
\hline $\begin{array}{l}\text { Author(s) \& } \\
\text { Year of } \\
\text { Publication }\end{array}$ & 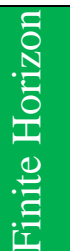 & 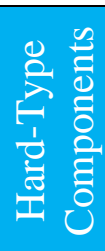 & 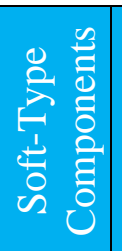 & 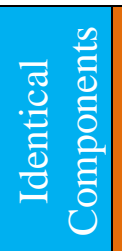 & 垔 & 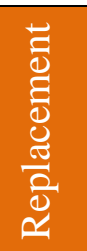 & 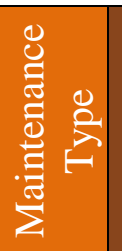 & 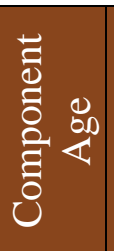 & 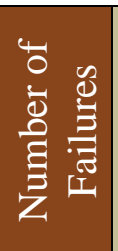 & $\begin{array}{ll}0 & 0 \\
0 & 0 \\
0 & 0 \\
0 \\
0 \\
0 \\
0 \\
0 \\
0 \\
0\end{array}$ & 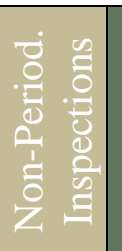 & 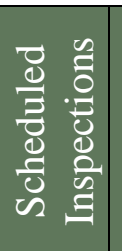 & 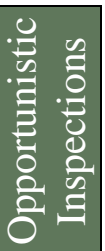 \\
\hline $\begin{array}{l}\text { Laggoune et } \\
\text { al. } \\
(2009)\end{array}$ & & & & $\checkmark$ & & $\checkmark$ & & $\checkmark$ & & $\checkmark$ & & $\checkmark$ & $\checkmark$ \\
\hline $\begin{array}{l}\text { Taghipour } \\
\text { et al. } \\
\text { (2010) }\end{array}$ & $\checkmark$ & $\checkmark$ & $\checkmark$ & & $\checkmark$ & & & $\checkmark$ & & $\checkmark$ & & $\checkmark$ & \\
\hline $\begin{array}{l}\text { Taghipour } \\
\& \text { Banjevic } \\
(2011)\end{array}$ & $\checkmark$ & & $\checkmark$ & & $\checkmark$ & $\checkmark$ & & $\checkmark$ & & $\checkmark$ & & $\checkmark$ & \\
\hline $\begin{array}{c}\text { Taghipour } \\
\& \text { Banjevic } \\
(2012 a)^{*}\end{array}$ & $\checkmark$ & $\checkmark$ & $\checkmark$ & & $\checkmark$ & $\checkmark$ & & $\checkmark$ & & $\checkmark$ & & $\checkmark$ & $\checkmark$ \\
\hline $\begin{array}{l}\text { Taghipour } \\
\& \text { Banjevic } \\
(2012 b)^{* *}\end{array}$ & $\checkmark$ & $\checkmark$ & $\checkmark$ & & 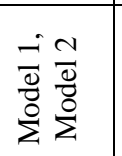 & $\frac{\sum^{2}}{\frac{\pi}{\Sigma}} \frac{\partial}{\partial}$ & & & & 吾 & & $\checkmark$ & $\checkmark$ \\
\hline $\begin{array}{l}\text { Golmakani } \\
\& \text { Moakedi } \\
(2012)\end{array}$ & $\checkmark$ & & $\checkmark$ & & $\checkmark$ & & & & & & $\checkmark$ & $\checkmark$ & \\
\hline $\begin{array}{l}\text { Gustavsson } \\
\text { et al. } \\
\text { (2014) }\end{array}$ & $\checkmark$ & $\checkmark$ & $\checkmark$ & & & $\checkmark$ & & $\checkmark$ & & $\checkmark$ & $\checkmark$ & $\checkmark$ & $\checkmark$ \\
\hline $\begin{array}{c}\text { Bjarnason et } \\
\text { al. } \\
(2014)\end{array}$ & $\checkmark$ & & & $\checkmark$ & $\checkmark$ & $\checkmark$ & & & & $\checkmark$ & & $\checkmark$ & $\checkmark$ \\
\hline $\begin{array}{c}\text { Taghipour } \\
\& \text { Kassaei } \\
(2015)\end{array}$ & $\checkmark$ & & & $\checkmark$ & $\checkmark$ & $\checkmark$ & & & & $\checkmark$ & & $\checkmark$ & $v$ \\
\hline $\begin{array}{c}\text { Bjarnason \& } \\
\text { Taghipour } \\
\text { (2016) }\end{array}$ & $\checkmark$ & & & $\checkmark$ & $\checkmark$ & $\checkmark$ & & & & $\checkmark$ & & $\checkmark$ & $\checkmark$ \\
\hline $\begin{array}{l}\text { Present } \\
\text { Work }\end{array}$ & $\checkmark$ & $\checkmark$ & $\checkmark$ & $\checkmark$ & $\checkmark$ & $\checkmark$ & $\checkmark$ & $\checkmark$ & $\checkmark$ & $\checkmark$ & $\checkmark$ & $\checkmark$ & $\checkmark$ \\
\hline
\end{tabular}

Notes:

* Taghipour, S., and Banjevic, D. (2012a)."Optimal inspection of a complex system subject to periodic and opportunistic inspections and preventive replacements," European Journal of Operational Research. V. 220 (3): 649-660.

** Taghipour S. and Banjevic D. (2012b). Optimum Inspection Interval for a System under Periodic and Opportunistic Inspections. IIE Transactions. 44 (11): 932-948. 


\subsection{Objectives of the Proposed Research}

The focus of the present research is on inspection and maintenance optimisation for multicomponent systems. Many publications exist in the literature that consider either inspection, or maintenance optimisation of multicomponent systems. However, there seems to be a lack of studies on the simultaneous optimisation of inspection and maintenance for such systems. The main objective of the present research is to further the knowledge of inspection and maintenance optimisation for multicomponent systems.

Specifically, the outlined objective refers to multicomponent systems with different aspects, which have been discussed in the previous sections. Namely, the focus of the current research is on the multicomponent systems with finite planning horizon, featuring:

- Systems composed of hard-type and soft-type components (the latter with hidden failures);

- Systems composed of identical components for $k$-out-of- $n$ redundancy;

- Minimal repair and replacement maintenance actions;

- Age-based maintenance decision for hard-type components;

- Maintenance count-based (number of minimal repairs before replacement) decision for hidden soft-type components;

- Periodic and opportunistic inspections;

- Non-periodic and opportunistic inspections.

Less formally, in practical terms, the proposed objective may be expressed in answering the questions regarding a complex multicomponent system with a finite planning horizon, such as:

- How to treat different components during inspection and maintenance? How different is the treatment going to be and which implications this could have on the total (or total expected) costs of inspection and maintenance for the entire system?

- How to choose the optimal maintenance action for a particular component when taking into account the consequences of such choice for the entire system? Which choices of maintenance actions for the duration of the planning horizon (i.e. maintenance policy) do result in the minimal total expected cost of inspection and maintenance for the entire system? 
- How to optimise inspection of different components when inspections occur at regular time intervals (with possible exception of the last inspection interval; see 'Methodology' for more details), as well as whenever a system fails? What is the optimal periodic inspection time interval that results in the minimal total expected cost of inspection and maintenance for the entire system?

- How to optimise inspection of different components when inspections occur at irregular time intervals or whenever a system fails? What are the optimal non-periodic inspection times (policy) that results in the minimal total expected cost of inspection and maintenance for the entire system?

Based on the stated objectives, the goal is to obtain mathematical models, each pertaining to the particular combination of system types and assumptions. 


\section{Joint Optimal Maintenance and Inspection for Periodic $k$ - out-of- $n$ Systems}

The present chapter is based on the following papers:

Babishin V. and Taghipour S. (2016). Joint Maintenance and Inspection Optimization of a $k$ out-of- $n$ System. Proceedings of the Annual Reliability and Maintainability Symposium 2016, pp. 523-528. Published online at: http://dx.doi.org/10.1109/RAMS.2016.7448039.

Babishin V. and Taghipour S. Joint Optimal Maintenance and Inspection for a $k$-out-of- $n$ System. (2016). International Journal of Advanced Manufacturing Technology, vol. 87, no. 5, pp. 1739-1749. Published online at: http://dx.doi.org/10.1007/s00170-016-8570-z.

In the present chapter, a $k$-out-of- $n$ system with hidden failures is considered. The system is subjected to periodic inspections over a finite life cycle (planning horizon). When the cumulative number of hidden component failures accumulates to $n-k+1$, a system failure occurs. Every system failure presents an additional opportunity for inspection and, therefore, is called "opportunistic". The objective is to find the optimal periodic inspection policy and the optimal maintenance action at each inspection for the entire system. Three types of maintenance are considered: minimal repair, preventive replacement and corrective replacement. In view of the failures' being hidden, the maintenance decision is based on the optimal number of minimal repairs before replacement (of either type). Due to the unavailability of a closed-form solution, joint optimisation of inspection and maintenance policies resulting in the minimal total expected cost is performed using exhaustive search and genetic algorithm (GA), both with integer inspection period constraint, and a GA implementation with quasi-continuous inspection period. Although both exhaustive search and GA with integer inspection period provide identical results, the genetic algorithm presents a more efficient procedure and requires less computational time, which becomes more noticeable with increasing complexity of the problem, as in the case of GA with quasi-continuous inspection period.

Based on the simulation results, some insights are made regarding the system's operation and cost optimisation. Expressions are derived for the expected number of system failures in 
terms of the cost ratio and component failure intensity under certain assumptions. In addition, a criterion is derived for establishing acceptable level of expected system failures over the system's life cycle. This can be useful when designing the system or analysing its performance.

Keywords: Periodic and opportunistic inspection, $k$-out-of- $n$ system, maintenance, genetic algorithm, preventive replacement, hidden failure.

\subsection{Introduction and Background}

Redundant systems that require high levels of robustness, reliability, availability and performance are usually designed with $k$-out-of- $n$ system configuration (Barlow and Heidtmann [46]). In this configuration, functionality and operation of the system is not interrupted whenever one of its components fails, unless the number of failed components accumulates to $n-k+1$. Examples of $k$-out-of- $n$ systems include airplanes with multiple engines, multiple redundant control mechanisms, multiple pumps in hydraulic control systems, as well as multiple displays in the cockpit. Cars often have dual-contour brake lines to allow for the brakes to operate if one of the hydraulic lines fails. Thus, it can be seen that $k$-out-of- $n$ active redundancy is employed in many systems.

Protective devices, reserve, or safety equipment represent a special class of multicomponent systems. Some examples of these devices include surge-protective equipment, liquid-level alarms and batteries in infusion pumps. Protective devices are usually "latent", or dormant when the system they protect is in a working order. However, when the system fails, the protective equipment has to start operating, and if it is unable to perform, then its failure is revealed. Because of the lack of information about the state of such protective systems, the failures of individual components making up these systems are most often discovered at either an inspection, or upon the entire system's failure. For this reason, such failures are called "hidden" $[8,16]$. However, in the absence of feedback on the state of protective systems, it is difficult to establish a reasonable, let alone optimal policy for their inspection and maintenance. At the same time, letting such devices run to failure is simply not an option in many instances, such as, for example, hospital infusion pumps, since the risks, danger and potential consequences of letting such devices fail are unjustifiably high.

In order to minimise the risks of failure, frequent regular inspections of the system and its 
components may be recommended, especially if the duration of inspection is negligible compared to the planning horizon. This, however, would defy the purpose of a redundant system, since the redundancy is embedded in such a system often with precisely the purpose to avoid frequent inspections, due to either difficulty, inaccessibility, high cost, etc., and so this option is generally inappropriate. In order to find the balance between mitigating the risks of failure and sustaining a cost-effective inspection policy, the inspection times have to be optimised over the entire planning horizon.

Hidden failures in $k$-out-of- $n$ systems are discussed by Bjarnason et al. [19, 47, 48], where the authors develop a joint optimisation model minimising the costs of maintenance and inventory. Bjarnason and Taghipour [21] also use genetic algorithm to search through a threedimensional objective function and to jointly find the optimal maintenance and $(s, S)$ inventory policies for a k-out-of-n system with hidden failures. In previous work by Kassaei and Taghipour [10], Taghipour and Kassaei [43] and Taghipour [9], the authors propose models for finding the optimal inspection interval that would minimise the total expected cost over a finitetime life cycle in a $k$-out-of- $n$ load-sharing system, where hazard rates of the operational components increase with each subsequent failure. None of these works, however, considers joint optimisation of inspection and maintenance.

Opportunistic inspections and preventive maintenance have received some coverage in the literature (Barlow and Hunter [49], Gustavsson et al. [50], Badía et al. [51]). Wang and Pham [52] propose a multi-objective imperfect preventive maintenance policy for a one-component system with hidden failures. Laggoune et al. [53] propose an opportunistic multi-grouping maintenance plan based on replacement optimisation for a multi-component system with series configuration subject to random system failures. Tambe et al. [54] use genetic algorithm, simulated annealing and sequence heuristic methods to find the optimal maintenance decision for a model with opportunistic maintenance action and available time and system availability constraints. Moghaddam [55] uses genetic algorithms (GAs) to solve nonlinear mixed-integer optimisation problem and determine the Pareto-optimal preventive maintenance plans for a multicomponent system. These models, however, do not feature inspection optimisation.

Lust et al. [56] use exact and heuristic methods for finding the best maintenance action that maximises system reliability. Jamshidi and Esfahani [57] utilise non-dominated sorting genetic 
algorithm II (NSGA II) to optimise maintenance action for a series and cold standby system. The type of system they consider is, however, different from $k$-out-of- $n$ systems, which makes their analysis not entirely appropriate for the latter.

In Dagpunar's model of a multicomponent system [39], if the failed component's age exceeds a specified control limit, it is opportunistically replaced. He, however, makes no distinction between the types of components, and maintenance decision is based on component's age rather than its maintenance history.

There exists literature on repair limit policies, with some criteria used as replacement decisions including repair time (Dohi et al. [58]) and repair costs (Drinkwater and Hastings [59], Kapur and Garg [60], Chien et al. [61], Lai [62]). When such repair limits are exceeded, the system or components undergo replacement.

Zhu et al. [40] propose an opportunistic maintenance policy for multicomponent system with hard-type and soft-type components, for the case of maintaining offshore wind turbines. In their model, however, maintenance action for soft-type components is again dependent on the ages of the latter.

Cui and Li [41] consider a multicomponent system with opportunistic inspections and stochastically-dependent components and use a cumulative damage shock model to stochastically compare maintenance policies. Still, they consider only one type of components.

Opportunistic inspections for $k$-out-of- $n$ systems have also received some coverage in the literature. Huynh et al. [44] provide an opportunistic condition-based maintenance model for maintenance optimisation at the component level. Huynh et al. [45] also propose a multi-level decision-making model.

Preventive replacement opportunities following a Poisson process for block and age-based replacement models are considered by Aven and Dekker [42], while Block et al. [63] consider age-dependent minimal repair. Taghipour and Banjevic [14] optimise minimal repairs of hardtype and soft-type components over a finite planning horizon with opportunistic inspections in one of their models. Taghipour and Banjevic [43] consider optimal inspection policy for complex multicomponent systems with hard and hidden soft failures under both periodic and opportunistic inspections and minimal repair or replacement maintenance. In these models, 
however, the component's maintenance action is determined by the age-dependent probabilities and is not subject to optimisation.

The majority of researchers considers either one, or two types of maintenance action. For example, Taghipour and Banjevic [14] assume in their first model minimal repairs only. Fard and Zheng [22] consider a non-repairable multicomponent system, which implies that the only type of maintenance available to them is replacement. Makis and Jardine [23], Sheu et al. [64, 65], Sheu and Chang [66], Cui [67], Okumura [68] and Pham and Wang [69] consider a system subject to replacements, which incur a smaller cost if done prior to failure than after it. Such models bear, however, rather a theoretical significance than a practical one, since they only consider one type of maintenance action. Most multicomponent systems are designed to allow for repairs, since it is usually more economical to repair a system than to replace it with the new, or "as good as new" identical system [24]. For this reason, of more interest are models which consider a variety of maintenance actions, such as both minimal repair and replacement.

Lienhardt et al. [70] and Chien and Sheu [25] consider two types of maintenance action: minimal repairs and replacements (also called "perfect repairs") for one-component systems, while Taghipour and Banjevic $[8,15]$ consider these types of maintenance for multicomponent systems, but they only optimise for periodic inspection policies.

Based on the aforementioned, it can be seen that there is a gap in the literature related to determining the optimal maintenance action to be taken at each component failure in a multicomponent system. A typical analysis involves optimisation of either inspection, or maintenance policies, but rarely both. With optimisation of inspection policies, many authors use rather strong assumptions on the type of maintenance policy that is taken, e.g. its dependence on the age. Although not unreasonable, this assumption limits the scope and the practical application of such models.

The current research is an attempt to fill the gap existing in the area of joint inspection and maintenance optimisation for $k$-out-of- $n$ systems with hidden failures, periodic and opportunistic inspections and several types of maintenance action.

The further structure of the present chapter is as follows: Section 3.2 outlines the problem; Section 3.3 proposes a model for corrective maintenance (Model 1) and describes the simulation procedure used to obtain the optimal joint inspection and maintenance policies; Section 3.4 
proposes a model for preventive replacement (Model 2) along with the simulation procedure; Section 3.5 then provides numerical examples for each model; Section 3.6 discusses the numerical results and provides sensitivity analysis; finally, Section 3.7 summarises conclusions.

\subsection{Problem Description}

Consider a redundant $k$-out-of- $n$ system, where $k$ refers to the number of identical components required to be in working order, $n$ refers to the total number of identical components and $1 \leq k \leq n$. It is assumed that component failures are hidden and discoverable only upon inspection, but that they follow a non-homogeneous Poisson process (NHPP) with the power law intensity function $\lambda(t)=\frac{\beta}{\eta}\left(\frac{t}{\eta}\right)^{\beta-1}$, where $t$ is the age of the component.

Furthermore, it is assumed that the system is of a $k$-out-of- $n: G$ type, it is able to withstand failure of its $j^{\text {th }}$ component, $j=1,2, \ldots, n$, until $n-k+1$ components fail. Upon failure of $n-$ $k+1$ components, the entire system fails and incurs the system downtime penalty. Failure of one or more of the system's components also incurs a time-dependent per-component downtime cost as a result of the penalties associated with the components' failures.

The system's planning horizon (life cycle) $\omega$ is assumed to be finite. In addition, two kinds of inspection are considered: periodic and opportunistic. A periodic inspection occurs with frequency $\tau$ months at times $i \tau, i=1,2, \ldots, l, l \in \mathbb{N}$, with the final periodic inspection and maintenance occurring at time $\omega$ months (and constituting a renewal point). An opportunistic inspection occurs every time the system fails, i.e. whenever an opportunity is presented outside of the regular scheduled periodic inspections. All components are checked at each inspection, and those failed undergo maintenance.

The maintenance action is considered to belong to either a minimal repair, a corrective replacement, or a preventive replacement. A minimal repair does not affect the component's age and restores the failed component to the working state it was in just prior to failure. A corrective replacement applies to a failed component and may occur both at the time of periodic, or opportunistic inspections. A preventive replacement is performed only at a periodic inspection on a working component. Both types of replacement decrease the component's age to 0 , thus effectively making it "as-good-as-new". 
In the real world, automotive brake lines present such problem. Multiple (usually dual, i.e. 1-out-of-2) redundant brake lines in cars increase reliability and safety by ensuring that if only one hydraulic line remains operational, there is still a pair of diagonally-opposite front and rear wheels with working brakes. There are common recommendations provided by the automotive manufacturers as to the frequency of inspections of brake fluid levels and brake master cylinder assembly, which fits the framework of periodic inspections. For such system, both corrective and preventive maintenance models developed further in the present chapter can be applied.

Another application of the proposed models is to uninterruptible power supplies with active redundancy. For these systems, electrical power storage devices (e.g. batteries or supercapacitors) can be periodically maintained and preventively replaced, based on the number of minimal repairs (charging cycles).

These models can also be applied to power relays, where corrective maintenance or preventive replacement may be performed based on the number of previous malfunctions.

These models can also be applied to power relays, where corrective maintenance or preventive replacement may be performed based on the number of previous malfunctions.

Both inspection and maintenance actions are assumed to be perfect and have negligible duration. Once the system's operating time reaches time $\omega$, a new optimal periodic inspection interval and maintenance policy is found taking into account the states of all components.

The objective of the current work is to determine the optimal periodic inspection interval $\tau^{*}$ and the optimal maintenance policy $m^{*}$ to be used upon a component failure. The optimal inspection and maintenance policy is defined by the minimal total expected cost for the entire system over the planning horizon.

\subsection{Model 1: Corrective Replacement of Components at Periodic Inspections}

Since the objective is to optimise the inspection and maintenance policies based on their total expected costs, it is necessary to first formulate the costs. The total expected cost $E\left[C_{m, \tau}^{T}\right]$ of the maintenance policy with $m$ as the number of minimal repairs before replacement and $\tau$ taken as the periodic inspection interval can be formulated as following: 


$$
\begin{aligned}
E\left[C_{m, \tau}^{T}\right] & =l c^{I}+F(\omega, \boldsymbol{t}, k, n) c^{S D}+\sum_{j=1}^{n}\left(c^{M} M_{m, \tau, j}\left(\omega, t_{j}, k, n\right)+c^{R} R_{m, \tau, j}\left(\omega, t_{j}, k, n\right)\right. \\
& \left.+c^{D}\left(\omega-U_{m, \tau, j}\left(\omega, t_{j}, k, n\right)\right)\right),
\end{aligned}
$$

where $l$ is the number of periodic inspections in the cycle $\omega, c^{I}$ is the cost of one periodic inspection, $\boldsymbol{t}=\left(t_{1}, t_{2}, \ldots, t_{n}\right)$ is the vector containing the components' initial ages, $F(\omega, \boldsymbol{t}, k, n)$ is the expected number of system's failures, $c^{S D}$ is the system downtime penalty cost, $c^{M}$ is the cost of component minimal repair, $c^{R}$ is the cost of component replacement, $c^{D}$ is the cost of component downtime per unit time, $t_{j}$ is the initial age of component $j, M_{m, \tau, j}\left(\omega, t_{j}, k, n\right)$, $R_{m, \tau, j}\left(\omega, t_{j}, k, n\right)$ and $U_{m, \tau, j}\left(\omega, t_{j}, k, n\right)$ are the expected numbers of minimal repairs, replacements and expected uptime of component $j$, respectively. These expected values depend on $\omega$, the initial age $t_{j}$ of component $j$, the number of required redundant components $k$ and the total number of components $n$. It should be also noted that, although the ages of failed components are unknown, they are used in the simulation to generate failures from NHPP and to keep track of components' uptime. It can be reasoned that the objective function, expressed as the total expected cost, is a convex function. Refer to Appendix A for arguments pertaining to convexity of the objective function.

Since the components' ages at failure are unknown, there is a need to use another component characteristic on which to base the maintenance decision. Such characteristic is taken to be the number of minimal repairs before replacement $m$ for each component $j$. Although components are identical, not all of them may fail for the same number of times, and for this reason it is necessary to keep track of the number of minimal repairs performed on each component. This approach has been applied in the past for non-identical components of multicomponent systems with hidden failures in [71].

In order to obtain the optimal number of minimal repairs before replacement, one needs to know the expected number of times a component will have to be fixed, i.e. the expected number of its failures. Since all components are identical, we obtain the expected number of failures $E[\Phi]$ for one component in order to construct the upper bound for the expected number of failures. Assuming that a component's failure detection occurs immediately upon inspection, and that the failed component is always minimally repaired, the expected number of failures 
$E[\Phi]$ can be calculated over the system's life cycle as following:

$$
E[\Phi]=\int_{0}^{\omega} \frac{\beta}{\eta}\left(\frac{t}{\eta}\right)^{\beta-1} d t=\left(\frac{\omega}{\eta}\right)^{\beta} .
$$

However, due to the stochastic nature of component failures, the exact number of failures encountered by each component may vary. For this reason, it is feasible to construct an $\alpha \%$ confidence interval for $E[\Phi]$ from Poisson distribution as following:

$$
\begin{aligned}
& U C L=\min _{\varphi}\left\{\sum_{f=0}^{\varphi} P(\Phi=f) \geq 1-\left(\frac{1-\alpha}{2}\right)\right\}, \\
& L C L=\max _{\varphi}\left\{\sum_{f=0}^{\varphi} P(\Phi=f) \leq \frac{1-\alpha}{2}\right\},
\end{aligned}
$$

where $U C L$ is the upper confidence limit, $L C L$ is the lower confidence limit and $P(\Phi=f)=$ $\frac{(E[f])^{f} e^{-E[f]}}{f !}$ is the probability of observing $f$ as the expected number of failures over $\omega$. Allowing $m$ to take values from 0 to $U C L$, cases ranging from immediate replacement upon failure to replacement on $(U C L+1)^{\mathrm{st}}$ failure can be covered.

In order to find the optimal number of minimal repairs before replacement $m^{*}$ and the optimal inspection interval $\tau^{*}$, the corresponding optimisation problem can be formulated as following:

$$
\begin{aligned}
& \left(m^{*}, \tau^{*}\right)=\min _{m, \tau}\left\{E\left[C_{m, \tau}^{T}\right]\right\}, \\
& \text { s.t. : } 0 \leq m \leq U C L, \tau=1,2, \ldots, \omega,
\end{aligned}
$$

where $E\left[C_{m, \tau}^{T}\right]$ is the total expected cost of the maintenance policy defined by $m$ and the inspection policy defined by $\tau$.

The pair $\left(m^{*}, \tau^{*}\right)$ denotes the optimal number of minimal repairs before replacement for any component and the optimal inspection period for the system. Hence, this pair constitutes the optimal joint maintenance and inspection policy for a given system.

Because the components' ages at failure are unknown, no explicit formula is available for obtaining the required expected values. The latter are obtained instead numerically from a simulation model, which is outlined in the following section. 


\subsubsection{Simulation Model}

The simulation model is created to take $k, n, \omega, \tau, t_{j}, \beta, \eta, c^{M}, c^{R}, c^{D}, c^{I}$ and $c^{S D}$ as the inputs. The simulation model then generates the times to the first failure for all components $j$. Using the Weibull-distributed reliability function $\mathcal{R}_{j}(t, \beta, \eta)$ and a random number $b$ generated from a uniform distribution Uniform $(0 ; 1)$, the next time to failure $\chi_{j}$, given the current component's age $t_{j}$, is generated as follows:

$$
\chi_{j}=\eta\left(\left(\frac{t_{j}}{\eta}\right)^{\beta}-\ln (b)\right)^{1 / \beta}-t_{j} .
$$

The first failure time is compared with the time of the first scheduled (periodic) inspection, given the inspection interval $\tau$ and the life cycle length. If the generated failure time is smaller than the next periodic inspection time, then the number of failed components is increased by 1 until it reaches $n-k+1$, at which point the system fails and an opportunistic inspection occurs. At this moment, all failed components are detected, and if the total number of previous failures for each component is less than $m$, the component is minimally repaired; otherwise, it is replaced, and its cumulative number of failures is set to zero.

If a component's failure time is smaller than the periodic inspection time and the number of failures is smaller than $n-k+1$, then the failed component is fixed at the periodic inspection time.

At any event's time (i.e. a component's failure time, the system's failure time and a periodic inspection's time), the random variables to track the number of minimal repairs, replacements and downtime of component $j$, and the number of system failures are updated and the simulation clock is advanced to the next event's time. Also, at an event's time, the time to the periodic inspection, the time to the next failure, and the ages of the surviving components are revised accordingly to incorporate the passing of the simulation's time. When the clock reaches the end of life cycle, the simulation stops. The averages of the estimates for the random variables obtained from the simulation model executed multiple times provide $F, M_{m, \tau, j}, R_{m, \tau, j}$ and $U_{m, \tau, j}$.

In each simulation run, for a given pair of $(m, \tau)$, the maintenance decision is made: either the component is replaced, if $f>m$, or it is minimally repaired otherwise. The total expected 
costs $E\left[C_{m, \tau}^{T}\right]$ from the simulation model obtained for different values of $(m, \tau), 0 \leq m \leq U C L$, $\tau=1,2, \ldots, \omega$, are then compared, and $\left(m^{*}, \tau^{*}\right)$ resulting in the minimal expected cost is the joint optimal maintenance and inspection policy.

Thus, the intermediate output of the simulation is a total expected cost matrix $\boldsymbol{E}\left[\boldsymbol{C}_{\boldsymbol{m}, \boldsymbol{\tau}}^{\boldsymbol{T}}\right]=$ $\left[\begin{array}{ccc}E\left[C_{1,0}^{T}\right] & \cdots & E\left[C_{1, U C L}^{T}\right] \\ \vdots & \ddots & \vdots \\ E\left[C_{\omega, 0}^{T}\right] & \cdots & E\left[C_{\omega, U C L}^{T}\right]\end{array}\right]^{\top}$, in which for each transposed total expected cost value, its row index presents the periodic inspection interval $\tau$ running from 1 to $\omega$ months, and its column index presents the number of minimal repairs before replacement $m$ running from 0 to $U C L$.

The final objective is satisfied by searching the total expected cost matrix for the minimum value as following:

$$
E\left[C_{m^{*}, \tau^{*}}^{T}\right]=\min _{m^{*}, \tau^{*}}\left\{\boldsymbol{E}\left[\boldsymbol{C}_{\boldsymbol{m}, \tau}^{\boldsymbol{T}}\right]\right\}
$$

Thus, the final outputs from the proposed simulation model are: the optimal number of minimal repairs before replacement for any component $m^{*}$ and the optimal periodic inspection interval $\tau^{*}$.

\subsection{Model 2: Preventive Replacement of Components at Periodic Inspections}

One metric used almost universally across a variety of manufacturing, industrial and services sectors regardless of the type of the system analysed is the total cost. This is especially true for the fields of inspection and maintenance optimisation, where both reliability and availability of the systems are connected through a variety of costs, such as component and system downtime penalties, inspection and maintenance costs, etc. Thus, a natural choice for the objective function in the case of a $k$-out-of- $n$ system is to formulate it in terms of the total cost of the joint inspection and maintenance policy.

However, in order to have a deterministic closed formulation, it is necessary to know with certainty all of the relevant parameters of the system. In the present case, this does not apply, since the components' ages at failure are unknown. Furthermore, to describe the expected values of the system, a recursive formulation can be developed which does not have a closed-form solution, since it incorporates multidimensional integrals, whose computation is intensive and 
very cumbersome due to the required discretisation procedure. For this reason, the choice of the analysis approach is made in favour of stochastic programming and numerical Monte-Carlo simulation.

Given the uncertainty associated with failure occurrence, we formulate the total expected $\operatorname{cost} E\left[C_{m, \tau}^{T}\right]$ for the policy defined by $m$ as the number of minimal repairs before replacement and $\tau$ as the periodic inspection interval, as following:

$$
\begin{aligned}
E\left[C_{m, \tau}^{T}\right]= & l c^{I}+F(\omega, \boldsymbol{t}, k, n) c^{S D}+\sum_{j=1}^{n}\left(c^{M} M_{m, \tau, j}\left(\omega, t_{j}, k, n\right)\right. \\
& +c^{C R} C R_{m, \tau, j}\left(\omega, t_{j}, k, n\right)+c^{D}\left(\omega-U_{m, \tau, j}\left(\omega, t_{j}, k, n\right)\right) \\
& \left.+c^{P R} P R_{m, \tau, j}\left(\omega, t_{j}, k, n\right)\right),
\end{aligned}
$$

where $l$ is the number of periodic inspections in the cycle $\omega, c^{I}$ is the cost of one periodic inspection, $\boldsymbol{t}=\left(t_{1}, t_{2}, \ldots, t_{n}\right)$ is the vector containing the components' initial ages, $F(\omega, \boldsymbol{t}, k, n)$ is the expected number of system's failures, $c^{S D}$ is the system downtime penalty cost, $c^{M}$ is the cost of component minimal repair, $c^{C R}$ is the cost of component's corrective replacement, $c^{D}$ is the cost of component downtime per unit time, $t_{j}$ is the initial age of component $j, c^{P R}$ is the cost of component's preventive replacement, $M_{m, \tau, j}\left(\omega, t_{j}, k, n\right), \quad C R_{m, \tau, j}\left(\omega, t_{j}, k, n\right)$, $P R_{m, \tau, j}\left(\omega, t_{j}, k, n\right)$ and $U_{m, \tau, j}\left(\omega, t_{j}, k, n\right)$ are the expected numbers of minimal repairs, corrective replacements, preventive replacements and expected uptime of component $j$, respectively. The variables inside the brackets following the expected values' symbols indicate the parameters used to calculate these expected values for the simulation purposes. The convexity of this cost function is demonstrated in Appendix A.

The component's age, although used as a parameter to generate the expected values in the simulation, is not known in reality due to hidden component failures. For this reason, the age of a hidden component cannot be used for making maintenance decisions. Instead, this decision is based on the component's number of minimal repairs before replacement.

The component's optimal number of minimal repairs before replacement is determined from its expected number of failures. Because all components in a $k$-out-of- $n$ system are identical, it is necessary and sufficient to obtain the expected number of failures $E[\Phi]$ for just one 
component, subject to assumptions of (1) no delay in detecting a failure on inspection and (2) ages of failed components are unaffected by maintenance (i.e. failed components are always minimally repaired), as following:

$$
E[\Phi]=\int_{0}^{\omega} \frac{\beta}{\eta}\left(\frac{t}{\eta}\right)^{\beta-1} d t=\left(\frac{\omega}{\eta}\right)^{\beta} .
$$

Because of the uncertain nature of component failures, the exact number of failures may differ among the components. In order to get a general estimate on the upper bound of component failures, a confidence interval of width $\alpha$ is constructed from Poisson distribution for $E[\Phi]$ as following:

$$
\begin{aligned}
U C L & =\min _{\varphi}\left\{\sum_{f=0}^{\varphi} P(\Phi=f) \geq 1-\left(\frac{1-\alpha}{2}\right)\right\}, \\
L C L & =\max _{\varphi}\left\{\sum_{f=0}^{\varphi} P(\Phi=f) \leq \frac{1-\alpha}{2}\right\},
\end{aligned}
$$

where $U C L$ is the upper confidence limit, $L C L$ is the lower confidence limit and the probability of observing $\varphi$ as the expected number of failures over $\omega$ is given by $P(\Phi=f)=\frac{(E[f])^{f} e^{-E[f]}}{f !}$. By making $m=0, \ldots, U C L$, different cases from replacement-on-first-failure to replacement on $(U C L+1)^{\text {st }}$ failure are covered.

The set value of $m$ is then used as a maintenance decision criterion for establishing a maintenance policy, such that minimal repair is performed on every failure until the number of failures for a particular component reaches $m$. A replacement (either corrective, or preventive) is then performed on $(m+1)^{\text {st }}$ failure for the same component. Based on this, optimising a maintenance policy amounts to finding the optimal number of minimal repairs before replacement $m^{*}$.

At the same time, the frequency of inspections or, equivalently, the length of a time period between two successive inspections, has to be determined in an inspection policy. Because all components in a $k$-out-of- $n$ system are identical and indistinguishable, it is immaterial to know all the combinations of failed components. Thus, optimisation for both maintenance and inspection can be performed jointly to obtain the optimal number of minimal repairs before replacement $m^{*}$ and the optimal inspection interval $\tau^{*}$ as following: 


$$
\begin{aligned}
& \left(m^{*}, \tau^{*}\right)=\min _{m, \tau}\left\{E\left[C_{m, \tau}^{T}\right]\right\}, \\
& \text { s. t. : } 0 \leq m \leq U C L, \tau=1,2, \ldots, \omega,
\end{aligned}
$$

where $E\left[C_{m, \tau}^{T}\right]$ is the total expected cost of the maintenance policy defined by $m$, and the inspection policy defined by $\tau$.

Thus, the optimal bivariate maintenance and inspection policy $\left(m^{*}, \tau^{*}\right)$ can be jointly obtained for the components in a $k$-out-of- $n$ system.

The difficulty arising from the lack of explicit formulation associated with obtaining the required expected values for a system with hidden failures is resolved by means of a simulation procedure outlined in the following section.

\subsubsection{Simulation Model Procedure}

Simulation is used to take $k, n, \omega, \tau, t_{j}, \beta, \eta, c^{M}, c^{C R}, c^{P R}, c^{D}, c^{I}$ and $c^{S D}$ as inputs and generate the times-to-failure for each component $j, j=1,2, \ldots, n$. Given the current component's age $t_{j}$, a random number $b$ generated from a uniform distribution Uniform $(0 ; 1)$ and assuming the Weibull-distributed reliability function $\mathcal{R}_{j}(t, \beta, \eta)$, the time-to-failure $\chi_{j}$ is generated from the following expression:

$$
\chi_{j}=\eta\left(\left(\frac{t_{j}}{\eta}\right)^{\beta}-\ln (b)\right)^{1 / \beta}-t_{j} .
$$

The time evolution of the system is then determined from comparing the time-to-failure with the time of the approaching periodic inspection, given the periodic inspection interval length $\tau$ and the planning horizon of length $\omega$. If the next periodic inspection time is greater than the generated failure time, then the component's number of failures increases by 1 .

The outlined procedure is repeated until the number of failures reaches $n-k+1$ failures. At this point, the entire system fails, triggering opportunistic inspection, which identifies all of the failed components. If the cumulative number of failures for a component does not exceed $m$, it is minimally repaired; otherwise, the component is fixed by corrective replacement and the cumulative number of its failures is reset to zero.

If the next periodic inspection time is greater than the generated component failure time, but 
the number of failures is less than $n-k+1$, then the failed component is fixed at the next periodic inspection. If the component's cumulative number of failures does not exceed $m$, it is minimally repaired; otherwise, similarly to the previous case, the component is fixed by corrective replacement and the cumulative number of its failures is reset to zero.

If the next periodic inspection time is smaller than the generated component failure time, and if the cumulative number of failures for a working component at the time of a periodic inspection is $m$, then that component is preventively replaced at that periodic inspection and the cumulative number of its failures is reset to zero. Otherwise, if the component is found in the failed stated at the periodic inspection, and its number does not exceed $m$, it is minimally repaired; otherwise, similarly to the previous cases, the component is fixed by corrective replacement and the cumulative number of its failures is reset to zero.

At the time of any event, such as component failure, system failure, or periodic inspection, the number of minimal repairs, the number of corrective and preventive replacements, the downtime of component $j$, and the number of system failures are all updated and the simulation clock is advanced to the time of the next event. In addition, the time until the periodic inspection, the time until the next failure, and the ages of the surviving components are revised accordingly to reflect the passage of time. When the clock reaches the end of planning horizon, the simulation stops. If the simulation model is run for a large number of times, it provides the averages of the estimates for the random variables $F, M_{m, \tau, j}, C R_{m, \tau, j}, P R_{m, \tau, j}$ and $U_{m, \tau, j}$.

During each simulation run, the choice of the maintenance action is made according to a given policy $(m, \tau)$. By changing the values of $(m, \tau)$ in the ranges $0 \leq m \leq U C L, \tau=$ $1,2, \ldots, \omega$, the total expected costs $E\left[C_{m, \tau}^{T}\right]$ for each policy $(m, \tau)$ is obtained and saved in a total expected cost matrix $\boldsymbol{E}\left[\boldsymbol{C}_{\boldsymbol{m}, \boldsymbol{\tau}}^{\boldsymbol{T}}\right]=\left[\begin{array}{ccc}E\left[C_{1,0}^{T}\right] & \cdots & E\left[C_{1, U C L}^{T}\right] \\ \vdots & \ddots & \vdots \\ E\left[C_{\omega, 0}^{T}\right] & \cdots & E\left[C_{\omega, U C L}^{T}\right]\end{array}\right]^{\top}$. In $\boldsymbol{E}\left[\boldsymbol{C}_{\boldsymbol{m}, \boldsymbol{\tau}}^{\boldsymbol{T}}\right]$, the row index represents the periodic inspection interval $\tau$ running from 1 to $T$ months, and the column index represents the number of minimal repairs before replacement $m$ running from 0 to $U C L$ for each policy $(m, \tau)$.

Finally, in order to find the optimal joint maintenance and inspection policy $\left(m^{*}, \tau^{*}\right)$, the total expected cost matrix is searched for the minimum total expected $\cos t E\left[C_{m^{*}, \tau^{*}}^{T}\right]$ according 
to the following rule:

$$
E\left[C_{m^{*}, \tau^{*}}^{T}\right]=\min _{m^{*}, \tau^{*}}\left\{\boldsymbol{E}\left[\boldsymbol{C}_{\boldsymbol{m}, \tau}^{\boldsymbol{T}}\right]\right\}
$$

The optimal total expected cost $E\left[C_{m^{*}}^{T} \tau^{*}\right]$ identifies the optimal number of minimal repairs before replacement $m^{*}$ and the optimal periodic inspection interval $\tau^{*}$, which together represent the joint maintenance and inspection policy $\left(m^{*}, \tau^{*}\right)$, thus accomplishing the present objective.

The exhaustive search used for finding the optimal policy is very susceptible to the size of the problem, i.e. the number of periodic inspections considered, the upper confidence limit on the expected number of component failures, etc. For this reason, with larger planning horizons or components with higher failure rates, both the search space and the simulation time increase dramatically. Another case where the problem complexity increases is when considering a smaller periodic inspection interval, as in this case the number of periodic inspections also increases. All of these cases suggest the need for improving the efficiency and reducing the complexity of the computations.

One method to reduce the complexity and the search space of the problem is to decrease the number of total expected cost calculations. A powerful heuristic search method that can be used to do this is the genetic algorithm.

Thus, with the goal of improving the efficiency of the simulation and in order to reduce its running time, the following model implementations are proposed: one based on exhaustive search, another one incorporating heuristic search using GA (both assuming $\tau$ to be integer) and the third - quasi-continuous GA relaxing the assumption of integer $\tau$. The three approaches are further discussed below.

\subsection{Numerical Examples}

This section contains numerical examples for the two models previously discussed.

\subsubsection{Model 1: corrective replacement of components at periodic inspections in a k-out-of-n system}

A 2-out-of-5 redundant system with identical components is analyzed using the model described in the preceding section. The component parameters are given in Table 2. 
Table 2: Per-Component Parameters of the Power Law Intensity Function and Costs.

\begin{tabular}{ccccc}
\hline$\beta$ & $\begin{array}{c}\eta \\
\text { (months) }\end{array}$ & $\begin{array}{c}\text { Minimal } \\
\text { repair cost, } c^{M}\end{array}$ & $\begin{array}{c}\text { Replacement } \\
\text { cost, } c^{R}\end{array}$ & $\begin{array}{c}\text { Downtime penalty } \\
\text { cost } / \text { month, } c^{D}\end{array}$ \\
\hline 1.5 & 7.5 & $\$ 75$ & $\$ 200$ & $\$ 60$ \\
\hline
\end{tabular}

The cost of inspection and system downtime penalty are both assumed to be fixed $\left(c^{I}=\$ 50\right.$, $c^{S D}=\$ 550$, respectively). Following the method explained above, the model is simulated using 5,000 runs with $T=12$ months. Based on the previously-stated assumptions, Equation (2) is used to calculate $E[f]$ and Equation (3) is used to obtain UCL. Table 3 below shows the $90 \%$ confidence interval $(\mathrm{CI})$ on the expected number of failures for identical components.

Table 3: Upper confidence limit ( $\alpha=90 \%$ ) on the expected number of failures for identical components.

\begin{tabular}{cc}
\hline $\begin{array}{c}\text { Expected Number } \\
\text { of Failures }(E[f])\end{array}$ & $\begin{array}{c}90 \% \\
\text { Upper Confidence } \\
\text { Limit }(U C L)\end{array}$ \\
\hline 2.0239 & 5 \\
\hline
\end{tabular}

Using the value of the upper confidence limit for the expected number of failures for the components, we calculate $E\left[C_{m, \tau}^{T}\right]$, as given in Equation (1), for $m=0, \ldots, U C L$. The optimal number of minimal repairs before replacement $m^{*}$ and the optimal inspection period $\tau^{*}$ are then jointly obtained from Equation (4) by sorting through $E\left[C_{m, \tau}^{T}\right]$ calculated for the system. The results are provided in Table 4.

Table 4: Transposed total expected cost matrix (in $\$$ ) for different values of $m=0,1, \ldots, 5$ and $\tau=1,2, \ldots, 12$ months in a 2 -out-of-5 system.

\begin{tabular}{|c|c|c|c|c|c|c|c|}
\hline & & \multicolumn{6}{|c|}{ Number of Minimal Repairs before Replacement $m$} \\
\hline & & 0 & 1 & 2 & 3 & 4 & $5^{*}$ \\
\hline \multirow{12}{*}{$\begin{array}{l}\text { Periodic } \\
\text { Inspection } \\
\text { Interval } \tau \\
\text { (months) }\end{array}$} & 1 & 1605.90 & 1610.28 & 1606.00 & 1622.21 & 1619.08 & 1610.40 \\
\hline & $2^{*}$ & 1515.79 & 1516.45 & 1525.55 & 1518.06 & 1518.24 & $1508.17 * *$ \\
\hline & 3 & 1623.49 & 1594.06 & 1610.06 & 1604.80 & 1610.70 & 1609.39 \\
\hline & 4 & 1716.14 & 1717.07 & 1710.78 & 1729.57 & 1718.60 & 1732.11 \\
\hline & 5 & 1729.02 & 1739.89 & 1744.25 & 1745.83 & 1740.18 & 1743.09 \\
\hline & 6 & 1904.57 & 1918.17 & 1935.85 & 1926.67 & 1918.26 & 1911.46 \\
\hline & 7 & 1906.33 & 1917.98 & 1896.81 & 1898.85 & 1885.37 & 1902.30 \\
\hline & 8 & 1907.20 & 1910.25 & 1917.27 & 1922.72 & 1917.47 & 1917.29 \\
\hline & 9 & 1930.61 & 1935.82 & 1920.46 & 1924.38 & 1938.16 & 1942.54 \\
\hline & 10 & 1982.21 & 1984.59 & 1989.15 & 1990.81 & 1992.59 & 1981.67 \\
\hline & 11 & 2097.96 & 2093.93 & 2092.87 & 2080.63 & 2082.53 & 2099.48 \\
\hline & 12 & 2201.58 & 2178.08 & 2190.09 & 2188.84 & 2183.17 & 2195.34 \\
\hline
\end{tabular}

Note: * indicates the optimal values of the $\left(m^{*}, \tau^{*}\right)$ pair. ** indicates the minimum total expected cost (i.e. $E\left[C_{m^{*}, \tau^{*}}^{T}\right]$ ). 
Table 4 above presents the total expected cost matrix $\boldsymbol{E}\left[\boldsymbol{C}_{\boldsymbol{m}, \boldsymbol{\tau}}^{\boldsymbol{T}}\right]^{\top}$. For convenience, the output from the simulation can be also visualized by fitting a 3-dimensional surface to the total expected cost matrix (see Figure 1).

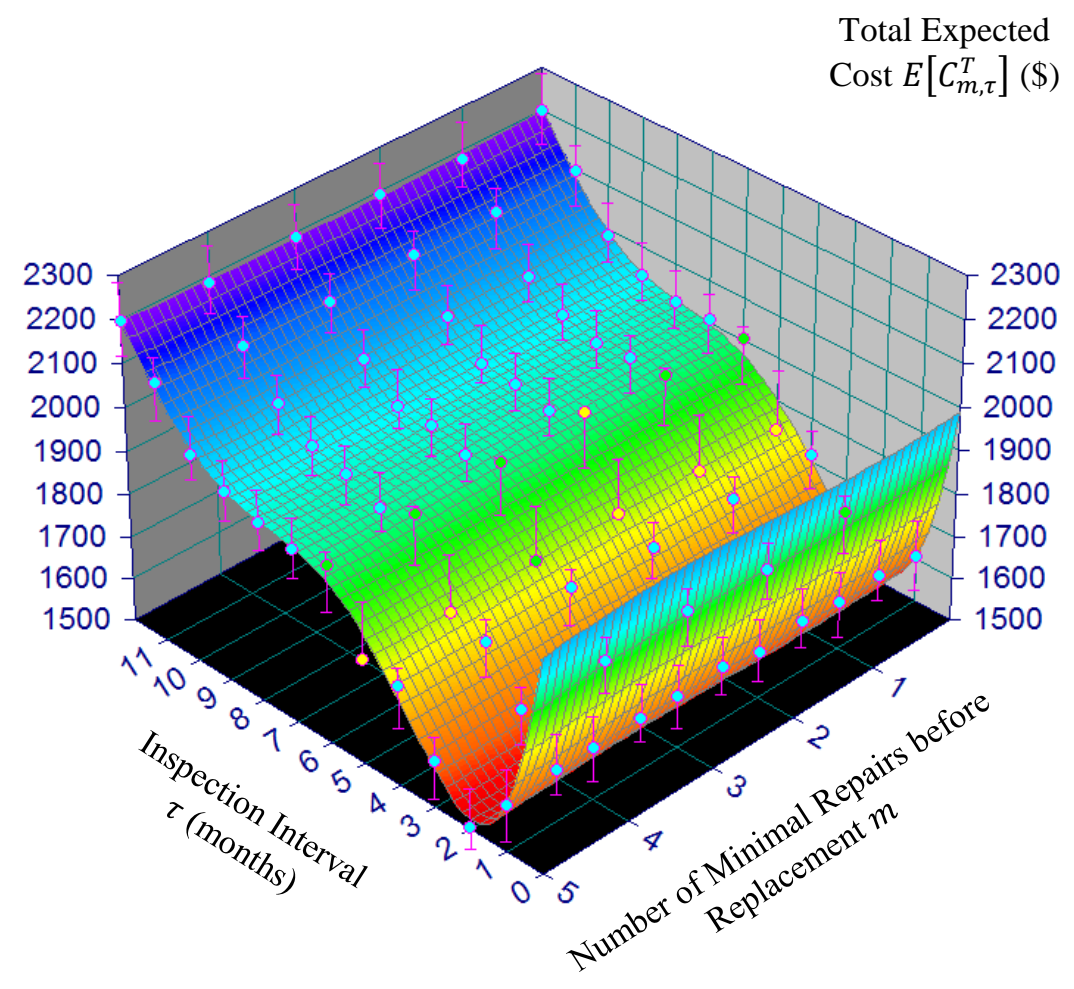

Figure 1: Surface Fitted to the Total Expected Cost Matrix for a 2-out-of-5 System.

Plotting the surface shown in Figure 1 allows for a better visualization of the relationship among the inspection interval, the number of minimal repairs before replacement and the total expected cost of the corresponding inspection and maintenance policies.

Looking at the 3-dimensional surface in Figure 1, it can be clearly seen that total expected cost is most sensitive to the periodic inspection interval length. It can be also seen that the lowest total expected costs occur at $\tau=2$ months. As can be ascertained from both Table 4 and Figure 1 , the minimum total expected cost is $\$ 1,508.17$ and its corresponding values of $\tau$ and $m$ are the optimal values of $\tau^{*}=2$ and 12 months and $m_{\tau}^{*}=5$. It can be also seen that any number of minimal repairs before replacement provides a near-optimal maintenance policy when periodic inspections are performed every 2 months.

Two additional cases to consider are a 1-out-of-5 system, which is equivalent to a parallel 
configuration, and a 5-out-of-5 system, which is equivalent to a series configuration. The results for these systems are provided in Table 5 and Table 6.

Table 5: Transposed total expected cost matrix (in $\$$ ) for different values of $m=0,1, \ldots, 5$ and $\tau=1,2, \ldots, 12$ months in a 1-out-of-5 (parallel) system.

\begin{tabular}{cccccccc} 
& & \multicolumn{6}{c}{ Number of Minimal Repairs before Replacement $m$} \\
\cline { 3 - 7 } & & 0 & 1 & 2 & 3 & 4 & $5^{*}$ \\
\hline & $2^{*}$ & 1586.63 & 1602.32 & 1605.81 & 1594.77 & 1592.24 & 1597.77 \\
& 3 & 1467.36 & 1436.80 & $1427.65 * *$ & 1435.37 & 1434.50 & 1439.09 \\
& 4 & 1543.32 & 1547.00 & 1552.82 & 1543.84 & 1554.70 & 1545.64 \\
Periodic & 5 & 1568.33 & 1588.93 & 1566.97 & 1572.60 & 1578.24 & 1572.61 \\
Inspection & 6 & 1739.35 & 1733.60 & 1719.03 & 1729.16 & 1731.16 & 1726.46 \\
Interval $\tau$ & 7 & 1709.20 & 1713.13 & 1711.60 & 1713.66 & 1700.74 & 1712.48 \\
(months) & 8 & 1726.84 & 1729.11 & 1731.86 & 1732.53 & 1723.89 & 1743.54 \\
& 9 & 1790.33 & 1778.00 & 1783.18 & 1785.45 & 1778.72 & 1778.88 \\
& 10 & 1878.20 & 1867.67 & 1873.97 & 1873.69 & 1888.28 & 1868.67 \\
& 11 & 2008.08 & 2008.97 & 2007.53 & 2012.52 & 2007.29 & 2009.21 \\
& 12 & 2129.99 & 2125.33 & 2122.63 & 2119.70 & 2131.25 & 2122.84 \\
\hline
\end{tabular}

Note: $*$ indicates the optimal values of the $\left(m^{*}, \tau^{*}\right)$ pair. ** indicates the minimum total expected cost (i.e. $\left.E\left[C_{m^{*}, \tau^{*}}^{T}\right]\right)$.

In Table 5, the minimal total expected cost of $\$ 1,427.65$ is incurred for the system when it is inspected every 2 months and when the failed components are replaced after $2^{\text {nd }}$ failure.

For the 5-out-of-5 system, in which all components have to be operational in order for the entire system to function, the results are shown in Table 6.

Table 6: Transposed total expected cost matrix (in $\$$ ) for different values of $m=0,1, \ldots, 5$ and $\tau=1,2, \ldots, 12$ months in a 5 -out-of-5 (series) system.

\begin{tabular}{cccccccc} 
& & \multicolumn{7}{c}{ Number of Minimal Repairs before Replacement $m$} & $5^{*}$ \\
\cline { 3 - 7 } & 1 & 6932.50 & 6943.58 & 6871.38 & 6933.78 & 6922.85 & 6914.75 \\
& 2 & 6624.50 & 6551.43 & 6601.30 & 6613.90 & 6592.10 & 6603.00 \\
& 3 & 6571.55 & 6520.63 & 6507.98 & 6524.63 & 6531.05 & 6521.63 \\
& 4 & 6470.33 & 6495.28 & 6475.20 & 6482.93 & 6472.40 & 6450.80 \\
Periodic & 5 & 6442.68 & 6501.30 & 6441.00 & 6450.33 & 6510.98 & 6496.50 \\
Inspection & 6 & 6422.63 & 6414.70 & 6430.93 & 6432.75 & 6415.83 & 6394.13 \\
Interval $\tau$ & 7 & 6426.20 & 6463.58 & 6404.00 & 6452.63 & 6390.03 & 6394.75 \\
(months) & 8 & 6358.50 & 6397.53 & 6418.48 & 6417.88 & 6397.45 & 6413.05 \\
& 9 & 6386.70 & 6470.75 & 6423.15 & 6407.28 & 6449.10 & 6419.80 \\
& 10 & 6438.23 & 6449.95 & 6390.30 & 6488.43 & 6394.28 & 6433.03 \\
& $11^{*}$ & $6355.33^{* *}$ & 6425.70 & 6392.38 & 6398.75 & 6431.35 & 6424.85 \\
& 12 & 6399.50 & 6363.98 & 6406.83 & 6403.63 & 6361.13 & 6393.33 \\
\hline
\end{tabular}

Note: * indicates the optimal values of the $\left(m^{*}, \tau^{*}\right)$ pair. ** indicates the minimum total expected cost (i.e. $\left.E\left[C_{m^{*}, \tau^{*}}^{T}\right]\right)$. 
Table 6 shows that the minimal total expected cost of $\$ 6,355.33$ for a series-configuration system consisting of five components is incurred whenever the system is inspected on the $11^{\text {th }}$ and $12^{\text {th }}$ months and if the failed components are replaced whenever they fail.

Comparing the results from Table 4, Table 5 and Table 6, it can be deduced that the lowest cost of inspection and maintenance is incurred for a 1-out-of-5 system, whereas the highest cost is incurred by the 5-out-of-5 system in series configuration. This is expected, since the total system downtime penalty incurred for a series system is much higher than that for a $k$-out-of- $n$, or a parallel system.

Looking at the periodic inspection interval length, it can be seen that the series-configuration system has the longest inspection interval of 11 months, compared to the 2 months optimal interval for 1-out-of-5 and 2-out-of-5 systems. This is likely due to the fact that the series system configuration is less reliable, since every component failure also causes the entire system to fail. For this reason, the optimal solution for such systems is to have opportunistic inspections on every component failure and reduce the number of periodic inspections to just 1 or 2 within the system's life cycle.

The optimal number of minimal repairs before replacement is greater for the case of 2-outof-5 system compared to the 1-out-of-5 system, since the number of failures, minimal repairs and replacements is generally greater for the 2-out-of-5 system. Thus, given the same frequency of periodic inspections, the 2-out-of-5 system has components failing for a greater number of times within the same time interval and, thus, necessitating component replacement sooner.

\subsubsection{Model 2: preventive replacement of components at periodic inspections in a $k$-out-of-n system}

Consider a redundant 3-out-of-5 system with parameters as given in Table 7.

Table 7: Per-Component Parameters of the Power Law Intensity Function and Costs.

\begin{tabular}{ccccc}
\hline$\beta$ & $\begin{array}{c}\eta \\
\text { (months) }\end{array}$ & $\begin{array}{c}\text { Minimal } \\
\text { repair } \\
\text { cost, } c^{M}\end{array}$ & $\begin{array}{c}\text { Corrective } \\
\text { Replacement } \\
\text { cost, } c^{C R}\end{array}$ & $\begin{array}{c}\text { Preventive } \\
\text { Replacement } \\
\text { cost, } c^{P R}\end{array}$ \\
\hline 1.5 & 7.5 & $\$ 75$ & $\$ 200$ & $\$ 180$ \\
\hline
\end{tabular}

In addition to parameters listed in Table 7, other costs include the fixed cost of inspection $c^{I}=\$ 50$, the per-component downtime penalty monthly cost $c^{D}=\$ 60$ and the system downtime 
penalty $c^{S D}=\$ 350$. It is assumed that all components are initially as-good-as-new.

Simulations are performed using exhaustive search and genetic algorithm techniques. Using both the former and the latter algorithms, the system is simulated for 5,000 runs with $\omega=12$ months on the same computer. The results obtained from each simulation are discussed below.

\subsubsection{1. $\quad$ Exhaustive search implementation}

For the exhaustive search implementation, Equation (8) is first used to calculate $E[f]$, followed by Equation (9) for calculating $U C L$. For the present example, the value of the $U C L$ was calculated to be 5 component failures, assuming a $90 \%$ confidence interval (CI) on the expected number of each component's failures.

Once the $U C L$ value has been obtained, the system's evolution was simulated, given the assumption of replacement (either corrective, or preventive) on $(m+1)^{\mathrm{st}}$ failure. Then, $E\left[C_{m, \tau}^{T}\right]$ was calculated from Equation (7) for $m=0, \ldots, U C L$. Finally, the array of $\boldsymbol{E}\left[\boldsymbol{C}_{\boldsymbol{m}, \boldsymbol{\tau}}^{\boldsymbol{T}}\right]$ values obtained for the system for the cases of different $m$ was sorted to jointly provide the optimal inspection period $\tau^{*}$ and the optimal number of minimal repairs before replacement $m^{*}$ (as represented in Equation (10) and (12)). See Table 8 below for details.

Table 8: Transposed Total Expected Cost Matrix (in \$) for Different Values of $m=0,1, \ldots, 5$ and $\tau=1,2, \ldots, 12$ Months in a 3-out-of-5 System.

\begin{tabular}{cccccccc} 
& & \multicolumn{6}{c}{ Number of Minimal Repairs before Replacement $m$} \\
\cline { 3 - 7 } & & 0 & 1 & 2 & 3 & 4 & $5^{*}$ \\
\hline & 1 & 9740.78 & 2383.53 & 1986.90 & 1808.52 & 1710.45 & 1676.69 \\
& $2^{*}$ & 4902.86 & 2185.28 & 1903.90 & 1746.34 & 1676.11 & $1658.80^{* *}$ \\
& 3 & 3579.12 & 2207.66 & 1978.73 & 1831.64 & 1765.80 & 1750.26 \\
Periodic & 4 & 3067.30 & 2294.54 & 2046.18 & 1909.95 & 1836.48 & 1825.84 \\
Inspection & 5 & 3088.20 & 2296.92 & 2064.92 & 1902.64 & 1849.47 & 1836.22 \\
Interval $\tau$ & 7 & 2743.42 & 2383.39 & 2155.73 & 1999.22 & 1939.54 & 1931.32 \\
(months) & 7 & 2758.43 & 2392.26 & 2148.75 & 1991.84 & 1940.53 & 1932.88 \\
& 8 & 2754.75 & 2372.45 & 2147.01 & 2000.27 & 1936.20 & 1931.16 \\
& 9 & 2787.67 & 2347.77 & 2153.59 & 1999.09 & 1952.41 & 1940.28 \\
& 10 & 2861.38 & 2346.86 & 2160.36 & 2007.89 & 1948.59 & 1942.17 \\
& 11 & 2973.70 & 2400.35 & 2233.75 & 2052.52 & 1993.66 & 1979.39 \\
& 12 & 2604.76 & 2414.26 & 2268.68 & 2101.41 & 2045.85 & 2042.88 \\
\hline
\end{tabular}

Note: * indicates the optimal values of the $\left(m^{*}, \tau^{*}\right)$ pair. ** indicates the minimum total expected cost (i.e. $\left.E\left[C_{m^{*}, \tau^{*}}^{T}\right]\right)$.

Table 8 above contains the total expected costs calculated for different values of the simulated $m$ and $\tau$. These data are also visualised in Figure 2. 
As can be seen from both Table 2 and Figure 2, the optimal total expected cost is $\$ 1658.80$, which occurs at the optimal periodic inspection policy $\tau^{*}=2$ months and the optimal maintenance policy $m^{*}=5$ minimal repairs before replacement.

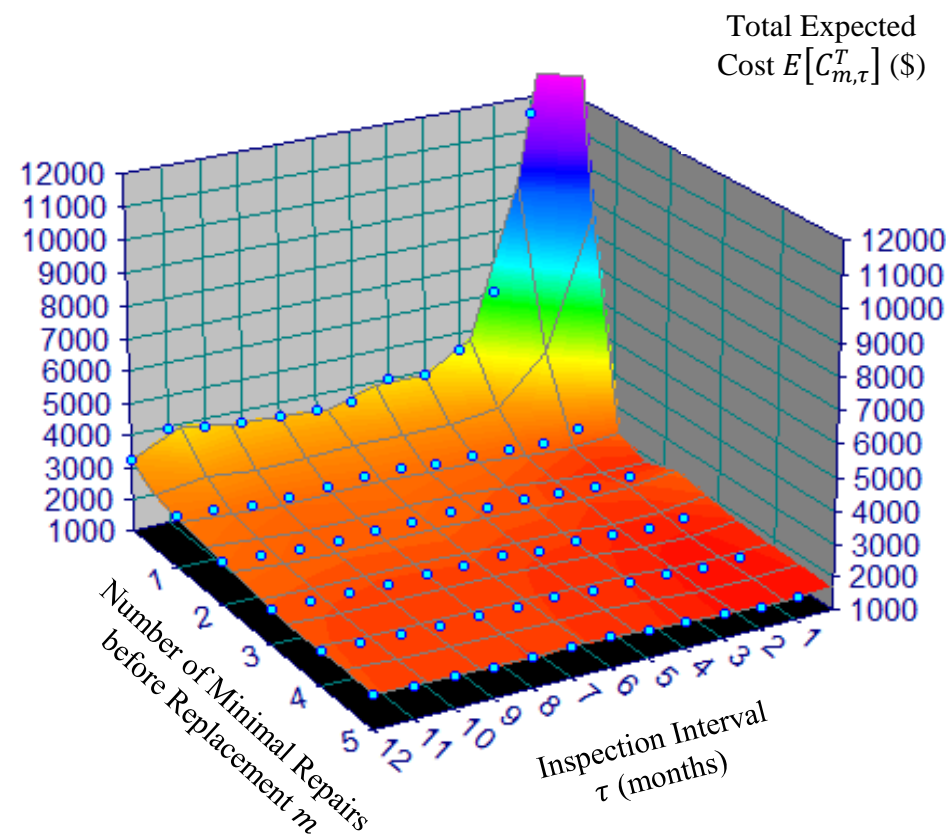

Figure 2: Continuous graphical representation of total expected cost matrix for a 3out-of-5 system.

Figure 2 also shows that periodic inspection interval has the greatest effect on the total expected cost, compared with the effect of the number of minimal repairs before replacement. The effect of the periodic inspection interval length is especially pronounced when going from $\tau=1$ to $\tau=2$ and then to $\tau=3$ for $m=0$ (i.e. "replace on each failure"). This is not surprising, given that the costs of corrective and preventive replacements are much higher than the cost of minimal repair, and thus increasing the number of replacements within the planning horizon sharply increases the total expected cost.

It can be also seen from Figure 2 that the variation among different inspection intervals at the same number of minimal repairs before replacement is relatively small. Thus, at $m=5$, almost any $\tau$ provides a total expected cost better than the total expected cost for the same $\tau$ at a different $m$.

\subsubsection{Genetic algorithm implementation with integer inspection period length}

In the genetic algorithm implementation, the values $m$ and $\tau$ used as inputs in the simulation 
are first generated from a random population of a given size. These generated decimal values are then converted to binary representation and encoded as genetic strings. These strings are then treated as being composed of "genes", which may be recombined, "excised", replaced, etc.

Several experimental runs were made, starting with the default population size of 20 and eventually reducing it to 4 . It was found that even the population of 4 was giving accurate results most of the time.

The fitness function used was similar to Equation (7), with lower total expected costs' providing higher fitness value than higher total expected costs.

For the selection procedure, "elite count" = 1 was used to guarantee that 1 individual survives to the next generation. A limit of 30 generations was used.

As alternative stopping criteria, in addition to the limits on the population size and the number of generations, a stall generation limit of 20 was used to put an upper bound on the number of generations over which the average relative change in the best fitness function value is calculated. In addition, another stopping criterion was the tolerance limit of $10^{-5}$. This criterion stops the algorithm, if the average relative change of the best function value is less than or equal to the specified value over the assigned number of stall generations.

The output and the results from running the genetic algorithm for the integer values of $\tau$ for 5,000 runs with the aforementioned settings is provided in Figure 3 below. 


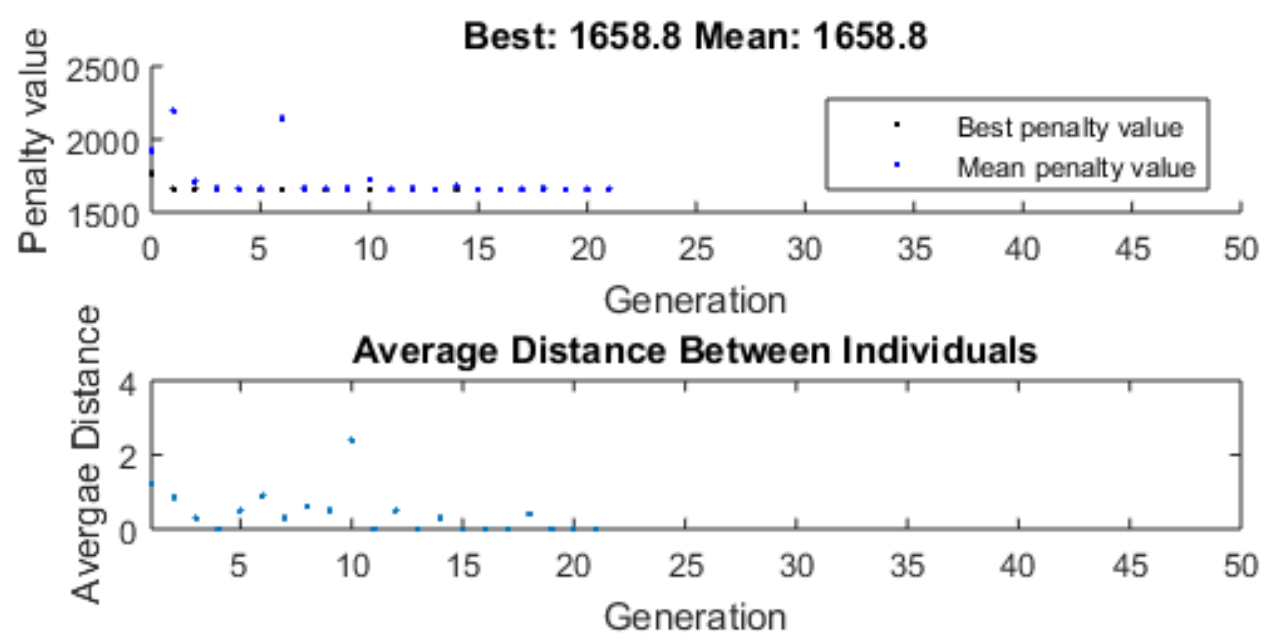

Best, Worst, and Mean Scores

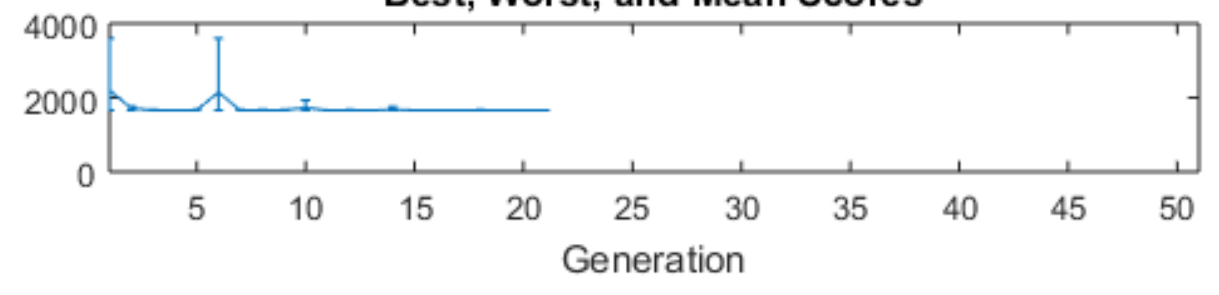

Figure 3: Integer $\tau$ genetic algorithm output and results.

The top graph in Figure 3 shows the output for the best and the mean $E\left[C_{m, \tau}^{T}\right]$ for each generation. As the number of generations increases, the GA-generated fitness function value gets closer to the optimal total expected cost and converges after about 14 generations.

The middle graph in Figure 3 displays the distances from each member of the population to its nearest neighbour. Generally, the smaller the distance, the faster the algorithm will converge.

The bottom graph in Figure 3 contains the best, worst and mean scores for the fitness function, i.e. the total expected cost. It can be seen that after approximately 10 generations, the differences among these three measures become relatively small.

The results from the genetic algorithm implementation matched those from the exhaustive search. The optimal number of minimal repairs before replacement and the optimal periodic inspection interval joint policies were found to be $\left(m^{*}=5, \tau^{*}=2\right)$, with the optimal total expected cost of $\$ 1658.80$.

The results obtained from both simulations are based on the minimum inspection period length $\tau$ of 1 month, which constitutes $1 / 12$ of the entire planning horizon length. In some cases, however, the minimum time between inspections may be smaller and/or the planning horizon 
may be much larger relative to the minimal inspection period length. In analysing such cases, rather than rescaling the problem to represent the minimal inspection period as an integer and the planning horizon as its integer multiple, the inspection period length may be thought of as being quasi-continuous, i.e. the integer constraint on the inspection period length can be relaxed. Thus, the value of $\tau$ is no longer limited to the integer number of months in this case, but may also take positive rational values.

\subsubsection{Genetic algorithm implementation with quasi-continuous inspection period length}

A genetic algorithm for the quasi-continuous case was also implemented. The results obtained from running the algorithm using the same seed, settings and constraints for 5,000 repetitions is shown in Figure 4 below.
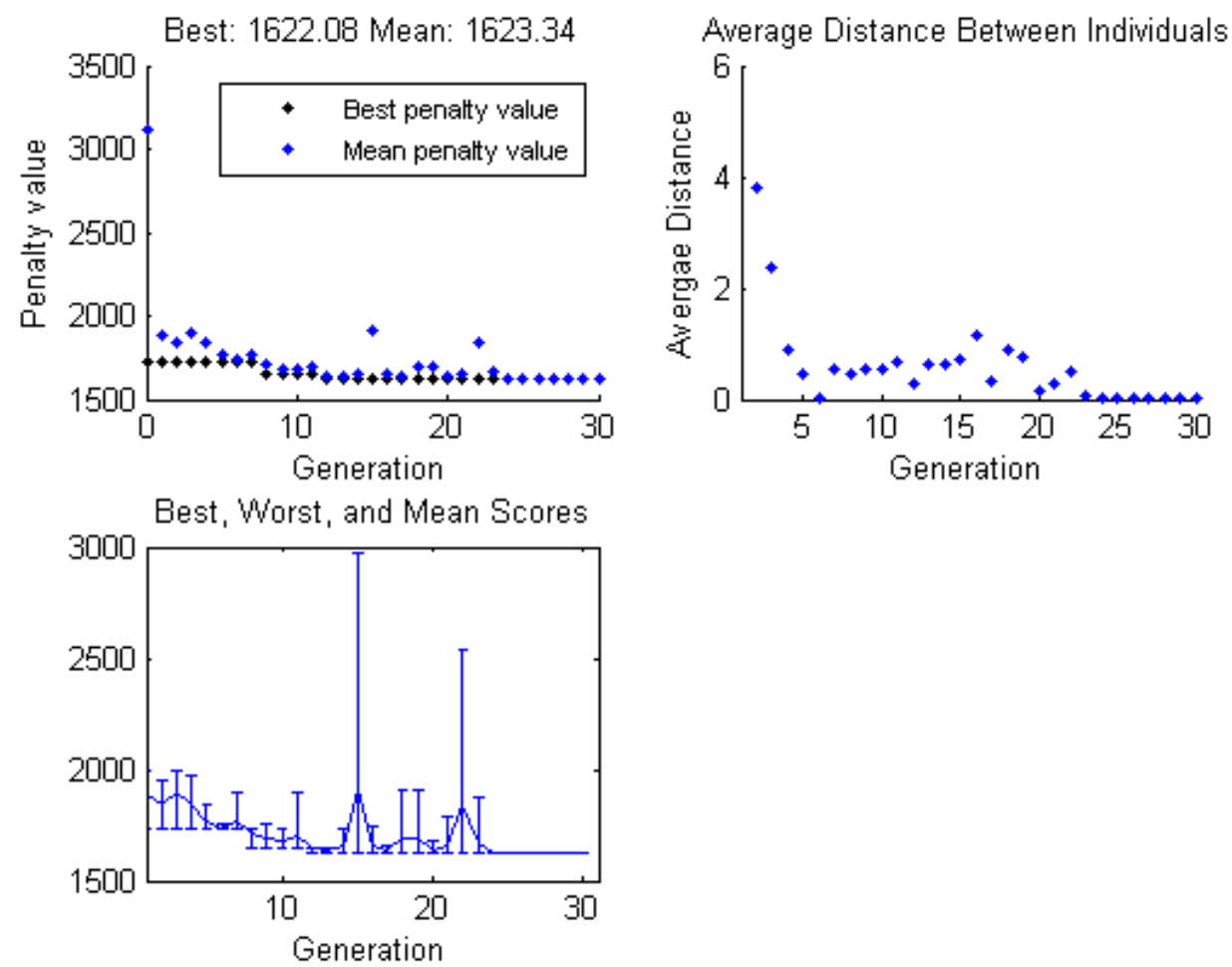

Figure 4: Quasi-continuous $\tau$ genetic algorithm output and results.

As can be seen from Figure 4, the optimal total expected cost of $\$ 1622.08$ was found, which is lower than those found from integer genetic algorithm and exhaustive search implementations. The joint optimal inspection and maintenance policies were found to be $\tau^{*}=$ 1.3335 months (40 days, based on a 30-day month) and $m^{*}=5$ minimal repairs before 
replacement, correspondingly, for the case of quasi-continuous inspection period.

The results from comparison of running times for 5,000 iterations between the genetic algorithm for integer $\tau$ and the exhaustive search suggest that with the running time of $58.6 \mathrm{~s}$ for the former vs. $250.9 \mathrm{~s}$ for the latter, the difference is more than 4-fold, with the GA's taking only about $23 \%$ of time required for the exhaustive search to find the same solution. Thus, the genetic algorithm implementation can be an efficient choice when analysing complex systems through simulation and has significant time advantages over the exhaustive search implementation.

It should be also noted that full advantage of the genetic algorithm implementation should become more evident with the increase in complexity of the problem. Although the quasicontinuous case took $4379.9 \mathrm{~s}$ to run, which is much greater than both $250.9 \mathrm{~s}$ for integer exhaustive search and $58.6 \mathrm{~s}$ for integer genetic algorithm, it is not surprising, since the step size was reduced from 1 month to ten thousandths of a month. Thus, it can be expected that in transitioning to the quasi-continuous case for the inspection period length, the time difference between the exhaustive search with the 4-decimal points step length and the genetic algorithm implementation is likely to be very dramatic. This suggests that the genetic algorithm is a powerful and versatile method to be applied to a wide range of inspection and maintenance optimisation problems.

\subsection{Discussion and Sensitivity Analysis}

Since hidden failures in multicomponent systems may go unnoticed for a long time and may accumulate to cause system failure in redundant configurations, the cost of system downtime may be quite large. In the present case, the system downtime penalty $c^{S D}$ represents the largest cost in the simulation. For this reason, it greatly affects the total expected cost and the latter's optimality, as can be ascertained from Figure 2. Therefore, the optimality of the joint maintenance and inspection policy $(m, \tau)$ is most sensitive to variation in the $\operatorname{cost} c^{S D}$.

Keeping all parameters constant and varying $c^{S D}$, its influence on the total expected cost

can be isolated. In Figure 5 below, $E\left[C_{m^{*}}^{T}, \tau^{*}\right] v s . c^{S D}$ is plotted for the optimal number of minimal repairs before replacement $m^{*}=5$ and the optimal $\tau^{*}=1$ and $\tau^{*}=2$, with all other parameters as previously indicated. 


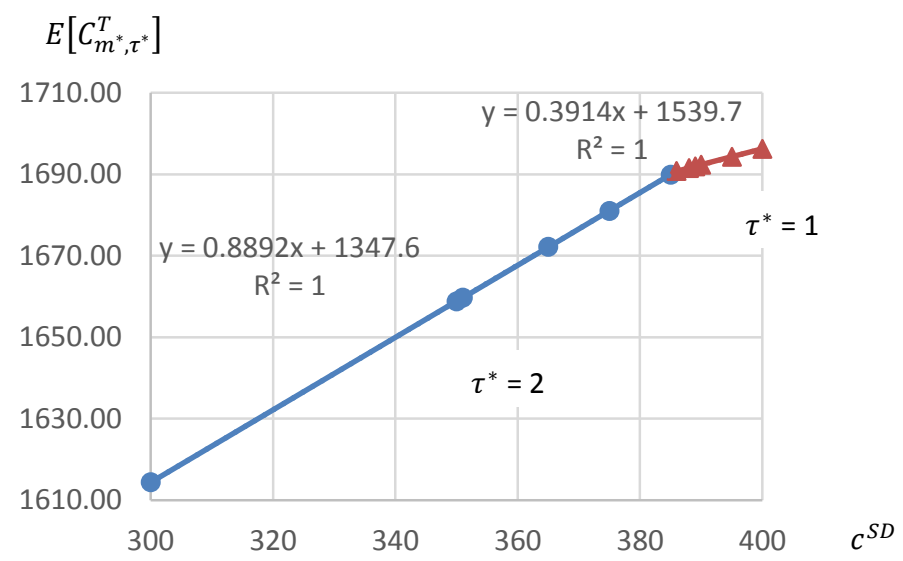

Figure 5: Expected total cost vs. system downtime penalty cost for $m^{*}=5$ and the other parameters as in Table 7.

As can be seen from Figure 5, the relationship between the total expected cost and the system downtime penalty is linear $\left(R^{2}=1\right)$ for both chosen optimal inspection periods. This is not surprising, since Equation (7) is linear in $c^{S D}$. The fitted equations expressing the linear dependency of $E\left[C_{m^{*}, \tau^{*}}^{T}\right]$ on $c^{S D}$ for optimal policies $\left(m^{*}, \tau^{*}\right)$ are:

$$
\begin{aligned}
& \text { For }(5,2): \quad E\left[C_{m^{*}, \tau^{*}}^{T}\right]=0.8892 c^{S D}+1347.6 \\
& \text { For }(5,1): \quad E\left[C_{m^{*}, \tau^{*}}^{T}\right]=0.3914 c^{S D}+1539.7
\end{aligned}
$$

Comparing Equation (13.1) and (13.2) with Equation (7), the following expressions can be deduced for $\left(m^{*}=5, \tau^{*}=2\right)$ :

$$
\begin{aligned}
& F(12, \mathbf{0}, 3,5)=0.8892=\frac{E\left[C_{m^{*}, \tau^{*}}^{T}\right]-1347.6}{c^{S D}} \\
& \sum_{j=1}^{5}\left(75 M_{5,2, j}(12,0,3,5)+200 C R_{5,2, j}(12,0,3,5)\right. \\
& \left.\quad+60\left(12-U_{5,2, j}(12,0,3,5)\right)+180 P R_{5,2, j}(12,0,3,5)\right) \\
& \quad+6 * 50=1347.6 .
\end{aligned}
$$

Similarly, for optimal policies $\left(m^{*}=5, \tau^{*}=1\right)$ :

$$
F(12, \mathbf{0}, 3,5)=0.3914=\frac{E\left[c_{m^{*}, \tau^{*}}^{T}\right]-1539.7}{c^{S D}},
$$




$$
\begin{aligned}
& \sum_{j=1}^{5}\left(75 M_{5,1, j}(12,0,3,5)+200 C R_{5,1, j}(12,0,3,5)\right. \\
& \left.\quad+60\left(12-U_{5,1, j}(12,0,3,5)\right)+180 P R_{5,1, j}(12,0,3,5)\right) \\
& \quad+12 * 50=1539.7 .
\end{aligned}
$$

Recalling from the definitions for Equation (7) that $F(\omega, \boldsymbol{t}, k, n)$ denotes the expected number of system's failures, it follows from Equation (14.1) that for the optimal policy $\left(m^{*}=\right.$ $5, \tau^{*}=2$ ), the given system is expected to fail 0.8892 times within the 12-months system's life cycle. Following the same logic, for the optimal policy $\left(m^{*}=5, \tau^{*}=1\right)$, the same system is expected to fail only 0.3914 times over its entire 12-months life cycle.

In addition, based on the definition of the expected number of system's failures for a NHPP over the system's life cycle:

$$
F(\omega, \boldsymbol{t}, k, n)=\int_{0}^{\omega} \lambda^{k, n}(t) d t,
$$

where $\lambda^{k, n}(t)$ denotes the system hazard or intensity function. Thus, Equation (15) directly connects a time-dependent stochastic expression for the expected number of system failures $F(\omega, \boldsymbol{t}, k, n)$ with the costs, as expressed in Equation (14.1) and (14.2).

To obtain the system hazard function, for simplicity, as the basis for further derivation in this section, we only consider the reliability formula valid for non-repairable systems with $k$ out-of- $n$ configuration. Thus, the reliability and the hazard functions presented further in this section describe the evolution of the system until first repair. Such derivation of first-failure system hazard function can be justified and particularly well-suited for highly reliable systems, where failure events are rare and, therefore, the proposed may be a good approximation to the true system hazard function.

Taking $\mathcal{R}(t)=\mathcal{R}=e^{-(t / \eta)^{\beta}}$ for individual components in a $k$-out-of- $n$ system, the system reliability $\mathcal{R}^{k, n}(t)$ until its first repair can be generally calculated as in Equation (16) below:

$$
\mathcal{R}^{k, n}(t)=\sum_{j=k}^{n}\left(\begin{array}{l}
n \\
j
\end{array}\right) \mathcal{R}^{j}(1-\mathcal{R})^{n-j}
$$




$$
=\sum_{j=k}^{n}\left(\begin{array}{c}
n \\
j
\end{array}\right) e^{-j\left(\frac{t}{\eta}\right)^{\beta}}\left(1-e^{-\left(\frac{t}{\eta}\right)^{\beta}}\right)^{n-j}
$$

The failure probability density function (or, equivalently, the negative derivative of the reliability function) until the first repair is given as:

$$
-\frac{d}{d t} \mathcal{R}^{k, n}(t, \beta, \eta)=\sum_{j=k}^{n}\left(\begin{array}{l}
n \\
j
\end{array}\right) \frac{\beta t^{\beta-1}\left(e^{\left(\frac{t}{\eta}\right)^{\beta}}-1\right)^{n-j-1}\left(j e^{\left(\frac{t}{\eta}\right)^{\beta}}-n\right) e^{-n\left(\frac{t}{\eta}\right)^{\beta}}}{\eta^{\beta}} .
$$

The system hazard function (failure rate) $\lambda^{k, n}(t)$ until the first repair (i.e. either an opportunistic, or a scheduled inspection) is then:

$$
\begin{aligned}
\lambda^{k, n}(t)= & -\frac{\frac{d}{d t} \mathcal{R}^{k, n}(t, \beta, \eta)}{\mathcal{R}^{k, n}(t, \beta, \eta)}= \\
= & \sum_{j=k}^{n}\left(\begin{array}{l}
n \\
j
\end{array}\right) \frac{\beta t^{\beta-1}\left(e^{\left(\frac{t}{\eta}\right)^{\beta}}-1\right)^{n-j-1}\left(j e^{\left(\frac{t}{\eta}\right)^{\beta}}-n\right) e^{-n\left(\frac{t}{\eta}\right)^{\beta}}}{\eta^{\beta}} \\
& \cdot\left(\sum_{j=k}^{n}\left(\begin{array}{l}
n \\
j
\end{array}\right) e^{-j\left(\frac{t}{\eta}\right)^{\beta}}\left(1-e^{-\left(\frac{t}{\eta}\right)^{\beta}}\right)^{n-j}\right)^{-1} .
\end{aligned}
$$

In designing or analysing the system for the number of failures, the boundary can be derived from Equations (15) and (16) for the expected number of system failures to remain less than 1 over the system's life cycle:

$$
\begin{gathered}
F<1 \Leftrightarrow \int_{0}^{\omega} \lambda^{k, n}(t) d t<1 \\
\int_{0}^{\omega} \sum_{j=k}^{n}\left(\begin{array}{l}
n \\
j
\end{array}\right) \frac{\beta t^{\beta-1}\left(e^{\left(\frac{t}{\eta}\right)^{\beta}}-1\right)^{n-j-1}\left(j e^{\left(\frac{t}{\eta}\right)^{\beta}}-n\right) e^{-n\left(\frac{t}{\eta}\right)^{\beta}}}{\eta^{\beta}}
\end{gathered}
$$




$$
\left(\sum_{j=k}^{n}\left(\begin{array}{l}
n \\
j
\end{array}\right) e^{-j\left(\frac{t}{\eta}\right)^{\beta}}\left(1-e^{-\left(\frac{t}{\eta}\right)^{\beta}}\right)^{n-j}\right)^{-1} d t<1
$$

For a highly reliable system with rare failures and, consequently, repair events, Equation (19) may be a good approximation to the general evolution of the system and not only for the system until its first repair.

The expressions given in Equation (19) may be used as a criterion for deciding on the appropriate planning horizon for the system. This expression can be useful for eliciting the acceptable level of risk associated with the system's failure. It also provides a quantifiable approach to setting the system's planning horizon, which, in most cases, is not a trivial task.

The preceding developments and Equations (13-19) allow simplifying the calculations pertaining to the system's analysis. This is an example of an advantage of a simulation approach, where it is able to provide not only a simplified approximation, but actually elicit an exact relationship and provide valuable insights for the system being analysed.

Figure 6 presents some of the values of the optimal $E\left[C_{m^{*}, \tau^{*}}^{T}\right]$ for the optimal policy $\left(m^{*}=\right.$ $\left.5, \tau^{*}=2\right)$. The values of $E\left[C_{m^{*}, \tau^{*}}^{T}\right]$ for this policy lie on the interval [\$1433.84, $\left.\$ 1689.93\right]$ and their range is $\$ 256.09$. The system downtime penalty $c^{S D}$ lies on the interval $[\$ 97, \$ 385]$, which translates into the range of $\$ 288$. Thus, for every $\$ 1$-increase in the system downtime penalty, the value of the total expected cost increases by approximately $\$ 0.89$ or, equivalently, for every $\$ 1$-increase in the total expected cost, the system downtime penalty increases by about $\$ 1.12$.

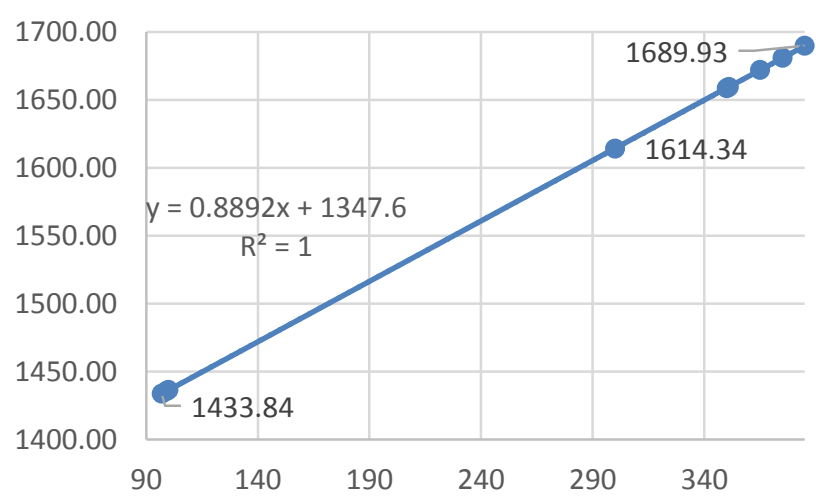

Figure 6: Range of system downtime penalty cost for which expected total cost remains optimal with $m^{*}=5, \tau^{*}=2$ and all other parameters as in Table 7 . 
Similar linear trend exists for all the values of $\tau^{*}$, as listed in Table 9 below.

Table 9: Ranges of system downtime penalty cost and total expected cost with the corresponding values of $\tau^{*}$ and $F(\omega, \boldsymbol{t}, k, n)$ for $m^{*}=5$ and other parameters as in Table 2 .

\begin{tabular}{lccc}
\hline$c^{S D}$ Range & $\mathrm{E}\left[\mathrm{C}_{\mathrm{m}^{*}, \tau^{*}}^{\mathrm{T}}\right]$ Range & $\tau^{*}$ & $F(\omega, \boldsymbol{t}, k, n)$ \\
\hline$[386, \infty)$ & {$[1690.78, \infty)$} & 1 & 0.3914 \\
{$[97,385]$} & {$[1433.84,1689.93]$} & 2 & 0.8892 \\
{$[35,96]$} & {$[1356.70,1432.92]$} & 3 & 1.2494 \\
{$[7,34]$} & {$[1315.04,1355.25]$} & 4 & 1.4892 \\
{$[0,6]$} & {$[1300.60,1313.32]$} & 12 & 2.1208 \\
\hline
\end{tabular}

As can be seen from Table 9 , the sensitivity of $E\left[C_{m^{*}, \tau^{*}}^{T}\right]$ to $\tau^{*}$ increases as $\tau^{*}$ decreases. This can be also ascertained from Figure 1, where the largest differences between $E\left[C_{m^{*}, \tau^{*}}^{T}\right]$ occur when transitioning between the smallest values of $\tau^{*}$.

As the value $\tau^{*}$ decreases, its sensitivity to $c^{S D}$ also decreases. This can be explained by the fact that higher system downtime makes it more costly for the system to remain in the failed state for a longer time, which is caused by the longer inspection period. Thus, $c^{S D}$ promotes smaller periodic inspection interval in order to retain the optimality of the total expected cost.

As $c^{S D}$ decreases, the sensitivity of $E\left[C_{m^{*}, \tau^{*}}^{T}\right]$ increases, which is indicated by the increasing magnitude of $F(\omega, \boldsymbol{t}, k, n)$.

Interestingly, the values of $F(\omega, \boldsymbol{t}, k, n)$ and $\tau^{*}$ both remain constant throughout the entire ranges of $c^{S D}$ and $E\left[C_{m^{*}, \tau^{*}}^{T}\right]$. This, again, can be explained by the linear relationship between $c^{S D}$ and $E\left[C_{m^{*}, \tau^{*}}^{T}\right]$ in Equation (7). Also, the fact that with longer duration of $\tau^{*}$, the value of $F(\omega, \boldsymbol{t}, k, n)$ increases, can be explained by the intuitive expectation that with the longer time intervals between inspections, more failures are expected to occur between inspections. Taking this into consideration, it can be seen that with lower system downtime penalty, the total expected cost may be optimal at less frequent inspections, which in turn allow for a higher number of expected system failures. Such non-trivial results re-emphasise the power and usefulness of the simulation models to consider a wide variety of scenarios and to provide the decision-makers with valuable insights regarding the given system.

The results for the optimal number of minimal repairs before replacement from Model 1 and Model 2 for a 2-out-of-5 and a 3-out-of-5 configuration, respectively, are presented in Table 10. 
Table 10: Total expected costs for Model 1 and Model 2 for each inspection period $\tau=$ $1,2, \ldots, 12$ months.

\begin{tabular}{ccc}
\hline $\begin{array}{c}\text { Inspection } \\
\text { period, } \tau \\
\text { (months) }\end{array}$ & $\begin{array}{c}\text { Model 1, } \\
\text { 2-out-of-5, } \\
E\left[C_{m^{*}, \tau^{*}}^{T}\right](\$)\end{array}$ & $\begin{array}{c}\text { Model 2, } \\
\text { 3-out-of-5, } \\
E\left[C_{m^{*}, \tau^{*}}^{T}\right](\$)\end{array}$ \\
\hline 1 & 1610.40 & 1676.69 \\
2 & $1508.17^{*}$ & $1658.80^{*}$ \\
3 & 1609.39 & 1750.26 \\
4 & 1732.11 & 1825.84 \\
5 & 1743.09 & 1836.22 \\
6 & 1911.46 & 1931.32 \\
7 & 1902.30 & 1932.88 \\
8 & 1917.29 & 1931.16 \\
9 & 1942.54 & 1940.28 \\
10 & 1981.67 & 1942.17 \\
11 & 2099.48 & 1979.39 \\
12 & 2195.34 & 2042.88 \\
\hline \multicolumn{3}{c}{ Note: * indicates the optimal costs. }
\end{tabular}

Since the tested system configurations differ for each model, the results do not allow for a close one-to-one comparison. A more informative comparison could be performed with the models applied to systems with identical configuration. However, there are still insights that could be gained from looking at the optimal costs for both models. It can be seen that the costs for Model 1 are lower for inspection periods of 1-8 months, even though the difference is generally no higher than $10 \%$. This can be ascribed to the fact that the 2-out-of-5 configuration tested for Model 1 is stochastically more reliable than 3-out-of-5 configuration tested for Model 2, which results in the lower expected costs of failures and repairs. For the inspection period length of 8-12 months, however, the total expected costs for Model 2 become increasingly lower than those for Model 1. Thus, even though the system configuration may be more reliable, the expected costs resulting from using the policy with preventive component replacement from Model 2 results in the lower expected costs as the inspection period length increases and the risk of failures rises. This proves the usefulness of considering preventive replacements in devising the joint inspection and maintenance models as opposed to relying only on the corrective maintenance actions in such analysis.

The limitations of the models arise from the assumptions of active redundancy, lack of operation over capacity, negligible inspection and maintenance duration, and deterministic inspection and maintenance costs. For example, the models do not account for "idling" of 
components, which occurs in standby-redundant systems, where some components enter operation only at failure of other components, until then acting as "spare parts". An example of a standby-redundant system is a set of wheels in a passenger car with a spare tyre, where the latter does not experience deterioration due to wear compared to the active tyres in contact with the road. The models proposed in this chapter also implicitly assume that components are not subject to load sharing when some of them fail, i.e. that the change in operating environment caused by failure does not affect the operating components' deterioration process. This is equivalent to components operating under full capacity while in active redundancy. The assumption of negligible inspection and maintenance duration is justifies when these actions take much shorter time compared to the planning horizon of the system, especially given the constant inspection and maintenance costs. However, it may become more restrictive if the inspection and maintenance costs depend on time, or if they are stochastic.

\subsection{Conclusion}

The present chapter considers the problem of determining the optimal inspection and maintenance policies for $k$-out-of- $n$ systems with hidden component failures under periodic and opportunistic inspections and finite planning horizon. Failures are assumed to follow a nonhomogeneous Poisson process (NHPP). The system fails when the number of component failures reaches $n-k+1$. At this time, an opportunity for inspection of all of the system's components is presented. Since component failures are hidden and their ages are unknown, the criterion used for the maintenance decision is the number of minimal repairs before replacement. Whenever a component reaches $(m+1)^{\text {st }}$ failure, it is either correctively, or preventively replaced. Preventive replacements occur only for operating components at the periodic inspection time, whereas corrective replacements are performed for the failed components at periodic and opportunistic inspections.

The total expected cost of inspection and maintenance policies is optimised jointly from simulation, since no closed-form solution is available due to hidden failures.

Numerical examples for corrective replacement model (Model 1) are provided for the cases of 1-out-of-5 (parallel), 2-out-of-5 and 5-out-of-5 (series) systems. As expected, the 1-out-of-5 system incurs the smallest optimal total expected cost of inspection and maintenance, while the 5-out-of-5 system incurs the highest optimal cost. This is due to the high total system downtime 
penalty, which is incurred more frequently for the 5-out-of-5 system. The optimal inspection period is longer for the 5-out-of-5 system, since the greater number of failures provides a greater number of opportunistic inspections, which reduces the need for frequent periodic inspections.

Three simulation implementations are proposed and compared for preventive replacement model (Model 2): one involving exhaustive search and the other two based on the genetic algorithm (GA). Similarly to the exhaustive search, the first GA implementation imposes integer constraint on the minimal periodic inspection length $\tau$, while the second GA implementation relaxes that constraint. The exhaustive search and the integer GA simulations provide identical results, but the genetic algorithm is found to reach the optimal solution much faster. The quasicontinuous GA requires much more running time, but provides joint inspection and maintenance policies resulting in yet lower total expected cost.

Furthermore, based on the simulation results, expressions are derived for the expected number of system failures in terms of the cost ratio, as well as in terms of the component failure intensity (assuming system evolution until its first repair). In addition, a criterion is derived on the planning horizon length for establishing acceptable level of expected system failures over the system's life cycle in terms of the system redundancy and component failure intensity. This can be useful when designing the system or analysing its performance.

As the future work, combining the joint optimisation of inspection, maintenance and inventory may be considered, with some cases including both integer and quasi-continuous inspection period. This problem may prove an especially suitable choice for genetic algorithm implementation owing to its large search space.

\section{Acknowledgements}

The author acknowledges the financial support from the Natural Sciences and Engineering Research Council (NSERC) of Canada for this research. 


\section{Optimal Maintenance Policy for Multicomponent Systems with Periodic and Opportunistic Inspections and Preventive Replacements}

The present chapter is based on the following paper:

Babishin V. and Taghipour S. Optimal Maintenance Policy for Multicomponent System with Periodic and Opportunistic Inspections and Preventive Replacements. (2016). Applied Mathematical Modelling, vol.40, no. 23-24, pp. 10480-10505. Published online at: http://dx.doi.org/10.1016/j.apm.2016.07.019.

In the present chapter, a system with components subject to soft and hard failures is considered. It is assumed that hard failures are revealed and fixed immediately and present an additional opportunity for inspection (opportunistic inspection), but soft failures are hidden and only corrected at periodic inspections. The objective is to find the optimal maintenance policy for all components and the optimal periodic inspection for the entire system. Two models are considered in this context. The first model features corrective maintenance (minimal repair or replacement) of hard-type and soft-type components with opportunistic inspections of soft-type components. The second model, in addition to the assumptions of the first model, allows for preventive replacement of hard-type components at periodic inspections. The maintenance decision is based on the optimal age before replacement for hard-type components and the optimal number of minimal repairs before replacement for soft-type components. A recursive equation is provided for deriving the required expected values. The optimal periodic inspection interval for the system minimising its total expected life cycle cost is found for both models using simulation, since no closed-form formulation is available due to hidden failures.

Keywords: Periodic inspection, opportunistic inspection, maintenance, optimisation, hidden soft failure, hard failure. 


\subsection{Introduction}

There are many multicomponent systems in various areas of industry, as well as in our life. Maintenance and inspection of the systems which rely on multiple parts, assemblies and components is very important, as failure of some systems may entail catastrophic consequences, incur huge costs and/or present danger to human life. Whenever a system either ceases to operate completely (fails), or stops operating as desired, various penalties, such as costs of downtime and lost work are often incurred. In order to minimise the excessive costs and undesirable consequences, the availability for such systems as, for example, medical devices, backup power generators, or computer network servers should be kept as high as possible [47, 48].

An overwhelming majority of multicomponent systems is specifically either designed, or may be assumed to be repairable, since it is usually more economical to repair a system than to replace it with the new, or "as good as new" identical system [24]. The main difference between the maintenance of simple (single-unit) and multicomponent (multi-unit) systems is that there usually exists some sort of dependency - either failure dependency, functional, structural, or economic dependency - among the individual components or subsystems of components in a multicomponent system [1]. Wang and Pham [72] study the optimal maintenance of a system consisting of several subsystems, assuming economic dependency among the components. Economic dependency in multicomponent systems is also surveyed by Dekker et al. [73]. Stochastic and economic dependencies and their influence on the periodic replacement policies are discussed within the multicomponent system's context by Ozekici [31]. Wang and Kuo [13] compare series systems with mixed (cold and warm) standby components using the mean time to failure, long-term availability and cost/benefit ratio. Zhang and $\mathrm{Wu}$ [29] consider a $k$-out-of$n$ system with perfect component repairs and imperfectly-repairable repair-equipment; they propose a model to minimise the expected cost rate of the repair-equipment, based on the optimal replacement policy for the number of repair-equipment failures. Shao and Lamberson [74], Taghipour [9] and L. Kassaei and Taghipour [10, 43] consider a $k$-out-of- $n$ load-sharing system where each failure increases the hazard rates for the remaining operational components. They develop a model for finding the optimal inspection interval minimising the total expected cost over a finite-time life cycle. 
Usually, the components of a multicomponent system may be conveniently classified by the type, or the mode of failure into hard and soft [75]. A component prone to hard failure is called a hard-type component, and that prone to soft failure - a soft-type component. If the same physical part of the system is subject to both soft and hard failures, it is treated as separate softtype and hard-type components. Failure of a hard-type component triggers the failure of the entire system and is, therefore, self-announcing, with the time of failure being known. Some examples of hard-type components are central processing unit in personal computers, fuse and display in infusion pumps, and ignition distributor wiring in automotive electronic ignition systems. Failures of soft-type components do not cause the immediate failure of the entire system, but usually reduce the system's reliability by eliminating redundancy and increasing the risk of damage or malfunction. Some examples of soft-type components are standbyredundant components (parallel processors, batteries, surge-protective equipment) and liquidlevel alarms in infusion pumps.

Various models have been proposed for optimal maintenance of multicomponent systems (Scarf [76], Wang and Christer [77], Zille et al. [78]), subject to various features and assumptions, such as hidden failures, finite planning horizon (Hartman and Murphy [79]), preventive replacements (Hyman [80], Seo and Bai [81], Chang [82]) and opportunistic inspections. Some work has been done to aggregate and summarise research in this area (Wang and Pham [72], Nakagawa and Mizutani [83]). For example, Wang [1] surveys different inspection and maintenance policies for both single-unit and multicomponent systems; however, his emphasis is on the systems consisting of one component. Cho and Parlar [2] provide another survey of literature, with most of the models having been developed over infinite time horizon. None of the models in these surveys, however, considers optimisation of both inspection and maintenance over a finite time horizon.

The planning horizon for a system usually represents its life expectancy. Examples of areas of industry where fixed and finite planning horizon is used include aircraft maintenance (see, for example, Sriram and Haghani [84]), pharmacology, medical devices with expiry date, etc. For example, aircraft parts usually have to be preventively repaired or replaced after a specific prescribed number of flight hours. Similarly, drugs and a vast majority of medical tools have an expiry date, after which they have to be replaced. 
Some systems, especially protective devices, usually contain components that are subject to hidden failures. A hidden failure can be defined as a failure not revealed during the normal operation of the system and discovered only at inspection [16, 85]. Hidden failures, such as failures of protective units in an integrated system of protective and protected units, are only revealed either at inspection, or whenever the protective unit is required to function, but is unavailable due to failure. Soft failures are similar to hidden failures, but the system is still able to function despite their presence. Sheu et al. [26] investigate hidden failures for singlecomponent systems and assume that the probability of failure depends on the number of previous repairs, while basing their maintenance policy on both the component's age-at-failure and its number of overhauls. Su [33] uses the supplementary variable technique to find the optimal inspection period that maximises the system's profit per unit time for a multi-mode system with a combination of hidden and self-announcing operating modes, where the period of inspection is a random variable. Bjarnason et al. $[19,20]$ consider hidden failures in the context of a $k$-out-of- $n$ redundant system and develop a joint optimisation model for minimising the total cost of both maintenance and inventory policies. Babishin and Taghipour [86] propose a joint optimisation procedure for minimising the total cost of both periodic and opportunistic inspections and corrective maintenance for a $k$-out-of- $n$ system with hidden failures. In another article, Babishin and Taghipour [87] consider a $k$-out-of- $n$ system with hidden failures under both periodic and opportunistic inspections, minimal repair, corrective or preventive replacement and find the optimal joint inspection and maintenance policies, as well as provide expressions for the expected number of system failures in terms of the cost ratio and component failure intensity. They also derive a criterion for calculating the acceptable number of system failures over the system's life cycle. Because of the lack of a closed-form solution, joint optimisation is performed using exhaustive search and genetic algorithm (GA) with either integer, or quasi-continuous inspection period. Bjarnason and Taghipour [21], they use genetic algorithm to search through a three-dimensional objective function to jointly find the optimal maintenance and $(s, S)$ inventory policies for a $k$-out-of- $n$ system with hidden failures. Unlike in $k$-out-of- $n$ systems, where all components are identical, in systems composed of various hardtype and soft-type components, each component may require a special treatment, which makes the analysis much harder. 
Gertsbakh [88] and Norros et al. [89] consider a two-component system weakened by failure of repairable components and analyse it in the context of semi-Markov processes. Anisimov and Gürler [90] propose threshold maintenance policy based on an approximate analytical method for a semi-Markov system composed of $n$ identical components subject to multiple unreliable states. Sun et al. [91], Tu et al. [92], Boudhar et al. [93] and Zhou et al. [94] consider joint component replacements and use heuristic and simulation optimisation methods. Jia [95] uses rule-based approach to opportunistic maintenance of life-limited components. Yu [96] surveys hidden semi-Markov models and outlines some popular applications. Generally, however, hidden semi-Markov models do not apply to the soft failures in the sense of the present chapter. For hidden semi-Markov models to be relevant, there have to be several states and transition probabilities defined for the soft-type components, which can describe the hidden degradation process of these components. In the present chapter, the age of the hidden soft-type components is unknown, and we are not looking at the degradation process of these components. Instead, once these components are inspected at either a periodic, or opportunistic inspection, their state (either functional, or failed) is deterministically and unambiguously identified.

Most of the models surveyed assign to each type of maintenance action some probability. Sheu and Griffith [27], for example, consider age-dependent probabilities for the type of maintenance (replacement, minimal repair, or imperfect repair). Complementary probabilities of minimal repair and replacement are considered by Murthy and Nguyen [97], Brown and Proschan [98] and Nakagawa and Yasui [99]. Chang et al. [100] and Chien and Sheu [25] assume that the probability of replacement depends on the system's age and the number of shocks tolerated since last replacement. Makis and Jardine [23] consider imperfect repairs, and in their model the probability of perfect maintenance depends on the number and time of imperfect maintenance actions in a cycle. More recently, Zhang and Zeng [101] propose a deterioration state space partitioning model for a system composed of multiple identical components, unlike the present chapter, where components are generally non-identical. They base their maintenance decision on the preventive maintenance threshold values, which are related to the deterioration state values. These authors, however, disregard the stochastic dependence of components by assuming independent deterioration of each component, thus treating failures as a homogeneous Poisson process, and associate probability with each maintenance type. No consideration is given for inspection optimisation. Instead of basing 
maintenance action on an age-dependent or state-dependent probability, a different approach is taken in the present chapter, where the optimal maintenance action is dependent directly on either the age, or the number of maintenance actions (minimal repairs) previously performed.

The majority of the available maintenance models structure the costs per unit time, for convenience, and optimise them over infinite planning horizon. Wang and Zhang [3] propose an optimal mixed bivariate policy to minimise the average cost rate, based on the critical reliability level and the number of system failures for a simple system. Zhang and Wang [4] and Lam [102] establish an optimal replacement policy based on the number of component failures for a series system with dissimilar components under a geometric process. They optimise their costs per unit time. Wu and Zhang [5] consider an infinite-horizon bivariate maintenance policy dependent on the interval length between preventive replacements and the number of component failures for a two-component cold-standby system subject to Poisson shocks. Coria et al. [6] develop an analytical optimisation method based on a new hazard function for imperfect preventive maintenance policy over an infinite planning horizon. Pan et al. [7] consider a preventive maintenance model with improvement factor and infinite planning horizon. Taghipour and Banjevic [8] propose models for finding the optimal inspection interval for a multicomponent system with hidden failures over both finite and infinite planning horizons. The focus of the present study is, however, on multicomponent systems with finite time horizon.

Opportunistic maintenance has also received an extensive treatment in the literature. Zhou et al. [12] consider opportunistic preventive maintenance optimisation for a multicomponent system with series configuration, which is equivalent to a multicomponent system consisting only of hard-type components, since failure of any component in a series configuration renders the entire system failed. Dagpunar [39] considers opportunistic replacement of a component in a multicomponent system, if the failed component's age exceeds a specified control limit. In his model, however, no difference is made between the types of components, and maintenance decision is based on component's age rather than its maintenance history. An opportunistic maintenance policy for multicomponent system with hard-type and soft-type components is proposed by Zhu et al. [40] for the case of maintaining offshore wind turbines. In their model, however, maintenance action for soft-type components is again dependent on the ages of the latter. Cui and $\mathrm{Li}$ [41] utilise stochastic comparison of maintenance policies in a cumulative 
damage shock model for a multicomponent system with opportunistic inspections and stochastically-dependent components. In one of the models in Taghipour and Banjevic [14], only minimal repairs of hard-type and soft-type components over a finite planning horizon are investigated for a multicomponent system with opportunistic inspections. Preventive replacement opportunities following a Poisson process for a framework spanning block and agebased replacement models are considered by Aven and Dekker [42]. Unlike the cases from the literature, in the present case, there is a choice of maintenance action to be taken at each component failure. Zhang and Zeng [103] model opportunistic maintenance based on the state space partition of a Markov process for a multicomponent system. However, the largest system they consider consists of three components, which is explained by the limiting calculation complexity of their approach, which is based on the stationary probability density determination for multi-unit system and the solution of a large system of linear equations for numerical integration. This reinforces the value and importance of simulation methods for modelling complex and/or large systems, both in theory and practice.

Bian and Gebraeel [104] consider a multicomponent system with stochastic component dependencies, but do not provide any optimisation models for either inspection, or maintenance optimisation. Vu et al. [105] consider multicomponent system maintenance in dynamic setting with time-limited opportunities. These authors, however, make no distinction between the components and their types of failure. Moreover, Vu et al. [105] do not optimise the decision criteria, whereas the methodology for maintenance action optimisation is proposed and discussed in the present chapter.

Taghipour and Banjevic [70] consider optimal maintenance policy and periodic inspection interval for complex multicomponent systems with hard and hidden soft failures under both periodic and opportunistic inspections and minimal repair or replacement. In their models, the choice of component maintenance action (minimal repair or replacement) is based on the agedependent probabilities and is not subject to optimisation. In the present chapter, unlike in [70], the maintenance decision is also subject to optimisation in addition to the inspection frequency [106]. In Model 3 proposed in this chapter, the optimal maintenance policy for hard-type components is based on the ages of these components. The optimal maintenance policy for softtype components is based on the number of minimal repairs before replacement for these components, similarly to the approach proposed by Park [30], Sheu [107], Sheu and Griffith 
[108] and Sheu et al. [109]. Model 3 assumes minimal repair and replacement as possible maintenance actions for both hard-type and soft-type components. In addition to the corresponding assumptions in Model 3, Model 4 also allows for preventive age-based replacement of hard-type components at periodic inspection times, as well as for opportunistic inspections of soft-type components at hard failure times. Recursive formulations are provided for finding the relevant expected values, but, as Vaurio notes in [36], it is not generally possible to obtain an analytical solution for the optimal inspection interval, even in the simpler case of optimising system availability regardless of the costs. For this reason, simulation is used to calculate the required expected values and to exhaustively search for the optimal solution in the case of a complex system.

To summarise the contributions, the present chapter provides a general methodology and two models for finding the optimal inspection period length and the optimal maintenance decisions for a complex multicomponent system with finite planning horizon. In the previous models by, for example, Taghipour et al. [24, 85], or Taghipour and Banjevic [8, 14, 70], no optimisation was done for maintenance action, and a failed component was just either minimally repaired, or replaced according to an age-dependent probability. None of the previous models by these, as well as other authors, have attempted to optimise both the maintenance action and the periodic inspection interval. In the proposed models, however, both the maintenance decision and the periodic inspection interval are optimised. Model 3 features corrective maintenance (minimal repair or replacement) of hard-type and soft-type components, and opportunistic and periodic inspections of soft-type components. Model 4, in addition to the assumptions listed for Model 3, features the possibility of preventive replacement of hard-type components at periodic inspections. In both models, the occurrence of hard failures may impact the expected number of soft failures, and thus, their expected number of minimal repairs, replacements and expected downtime. Consequently, these expected values influence the optimal periodic inspection interval. In Model 4, the costs of hard-type components can impact the optimal periodic inspection interval as well. In addition, general recursive mathematical formulations were developed separately for Model 3 and Model 4 for generating all of the expected values necessary for both models. These are presented in the appendices.

Overall, inspection and maintenance optimisation models can be used as valuable tools in providing the safe and reliable operation of various equipment. Such models can also have 
strong managerial implications, since in practice, it is usually important to justify and support managerial decisions with both qualitative and quantitative analysis in order to make them robust. Simulation models are particularly useful in this regard, as they can cover a great number of possible scenarios and provide the results both for a particular and the most general case. Using the proposed inspection and maintenance optimisation model, the decision-maker(s) gain an opportunity to observe the outcomes of their managerial decisions and to find the combination of decisions that is most likely to result in the greatest cost savings without sacrificing the required reliability and availability. For example, based on the model's output for a given system with particular component parameters, it may not require as frequent inspection as previously thought because of the accounted effect of the additional opportunistic inspections. This would result in cost savings, which would be especially significant, if the costs of inspection were particularly high. Thus, it can be seen that optimisation of inspection and maintenance decisions are a valuable asset for decision-makers.

The further structure of the present chapter is as follows: Section 4.2 provides the problem description; Section 4.3 contains the model formulation for the case of corrective hard-type component maintenance and opportunistic inspection of soft-type components; Section 4.4 modifies the previous model by allowing for preventive replacement of hard-type components at periodic inspections; Section 4.5 illustrates the models with numerical examples; Section 4.6 provides sensitivity analysis and discussion of some results; finally, Section 4.7 summarises the conclusions. Appendix B provides the mathematical formulations of the models described in Sections 4.3 and 4.4, and Appendix C lists the simulation algorithm used for the same model in the form of a flowchart.

\subsection{Problem Description}

The main problem considered in the present chapter is finding the optimal inspection and maintenance policy for a multicomponent system prone to experiencing hard and soft failures. For example, in general infusion pumps found in hospitals, fuses and displays are considered as hard-type components, and liquid-level alarm and battery - as soft-type components.

The following assumptions are made regarding such system (assumptions pertinent to Model 3 are further identified by designation "M.1.\#”, and those pertinent solely to Model 4 by “M.2.\#”, where "M." stands for “model”): 
M.1.1: Each of the individual components of the system is assumed to belong to one of the two possible types: soft type, or hard type. The total number of soft-type components $s$ in the system is $n_{1}, s=1,2, \ldots, n_{1}$, and the total number of hard-type components $h$ is $n_{2}, h=1,2, \ldots, n_{2}$.

M.1.2: The components' failure times are assumed to follow a non-homogeneous Poisson process (NHPP) with the power law intensity function (hazard function) $\lambda_{j}(t)=$ $\frac{\beta_{j}}{\eta_{j}}\left(\frac{t}{\eta_{j}}\right)^{\beta_{j}-1}$, where $t$ is the age of component $j$ and $j=1,2, \ldots, n_{1}+n_{2}$.

M.1.3: By definition, soft failures are discovered only at inspections. Therefore, the ages at failure of soft-type components are unknown.

M.1.4: Inspections are classified as being either periodic, or opportunistic. Periodic inspections occur with the periodic interval of length $\tau$ over a finite planning horizon $\omega$ at times $i \tau, i=1,2, \ldots, l, l \in \mathbb{N}$. Opportunistic inspections are incurred whenever a hard-type component fails.

M.1.5: Repairs or replacements are performed only on failed components.

M.1.6: Both inspections and replacements are assumed to be perfect and have negligible duration.

M.1.7: Replacements put the component in the "as good as new" state, resetting the component's age to 0 , while minimal repairs return the component to the state it was in just before failure and do not affect the component's age.

M.1.8: All of the components are inspected at the end of the operating cycle (planning horizon) $\omega$ in preparation for the next planning cycle, and all failures are rectified. The new optimal periodic inspection interval for the next cycle can then be found by taking into account the current ages of all components.

Whenever a hard-type component fails, it is inspected along with all soft-type components. Each failed component is then either minimally repaired, or replaced. Therefore, hard failures 
create more opportunities for inspecting soft-type components and, consequently, influence the number of minimal repairs, replacements and downtime of the latter (see Figure 7).
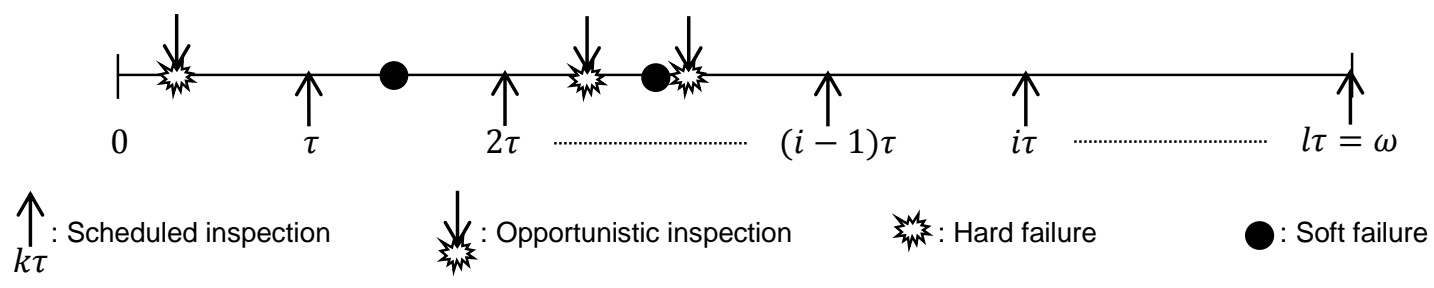

Figure 7: Sample soft and hard failures with periodic (scheduled) and opportunistic (unscheduled) inspections within cycle $\omega$.

It should be noted that the downtime of the failed soft-type components negatively influences the performance of the entire system. To reflect this, the following additional assumption is made:

M.1.9: Downtime penalty cost is incurred per unit time by each of the failed soft-type components.

As a practical example of a system possessing the properties and assumptions given in the current section, programmable infusion pumps were considered. The Weibull distribution parameters for hard-type and soft-type components were based on the data collected from observing the use of programmable infusion pumps during earlier work, published in part by Taghipour et al. in [45]. Other suitable systems include oil pipelines, automobiles, computer systems, carbon monoxide detectors, uninterruptible power supply units, etc.

The objective of the present study is to determine the optimal maintenance actions that should be taken for a failed component (either soft-type, or hard-type) and to find the optimal periodic inspection interval $\tau^{*}$. This is equivalent to establishing the optimal maintenance policy and inspection interval for the components of the system. It should be noted that it is not enough to simply inspect the soft-type components more frequently than the hard-type components because the former's failures are hidden. The goal of the present research is to obtain policies that are globally-optimal for the entire system, and not necessarily for specific components or groups thereof. For example, the parking brake in a car may be considered as a soft-type component, since its failure (e.g. cable rupture) is noticeable only when its functioning is required, such as when preventing vehicle roll when stopped on a slope. However, it is unreasonable to expect that parking brake cable be inspected, for instance, before every start of 
the vehicle. It is much more economical (in the sense of time, at least) to have the parking brake inspected when the car undergoes periodic scheduled maintenance (e.g. oil change), or at the failure of any of its hard-type components (e.g. transmission), which presents an opportunity for inspection of the soft-type components as well.

In obtaining the total expected per-component cost for a given number of minimal repairs before replacement $n_{j}$ and a given inspection period, the following assumption is made:

M.1.10: Component $s$ is replaced after $m_{s}$ minimal repairs.

In order to obtain an upper bound for $m_{s}$, an $\alpha$-level confidence interval (CI) for the expected number of failures over $\omega$ is constructed separately for each soft-type component, making use of the following two assumptions:

M.1.11: A soft failure is immediately detected upon inspection.

M.1.12: The failed component is always minimally repaired.

For Model 4, another assumption is made in addition to the above-listed assumptions pertaining to Model 3:

M.2.1: Preventive replacements of hard-type components are possible at periodic inspection times for the system under consideration.

The following sections discuss both models and provide the optimal inspection and maintenance strategies, followed by a numerical example to illustrate the proposed methodology.

\subsection{Model 3: Corrective Maintenance of Hard-Type Components and Periodic and Opportunistic Inspections of Soft-Type Components}

In this section, a 3-step method is proposed for finding the optimal maintenance actions after failures and the optimal inspection interval for the system, taking into account the fact that soft failures are hidden and the ages of the soft-type components at the time of failure are unknown.

At the first stage, the optimal time to replacement resulting in the minimal expected cost for the component per unit time is found for each hard-type component. At the second stage, the optimal number of minimal repairs before replacement resulting in the minimal total expected cost for the component over the system's life cycle is found for each soft-type component, since 
its age at failure is unknown. Finally, at the third stage, given the optimal maintenance actions for hard-type and soft-type components, the optimal periodic inspection for the entire system resulting in the minimal expected cost for the system over its life cycle is found.

The details for the three stages are provided below.

\subsubsection{Stage 1. Obtaining the optimal replacement ages for hard-type components}

Given that the ages of the hard-type components are always known, the model described by Park in [30], for which the renewal theory was used, can be utilised to obtain the optimal replacement age $\zeta_{h}^{*}$ of hard-type component $h$ (assuming hazard function from a Weibull distribution) as follows:

$$
\zeta_{h}^{*}=\eta_{h}\left(\frac{c_{h}^{R}}{\left(\beta_{h}-1\right) c_{h}^{M}}\right)^{1 / \beta_{h}}
$$

where $c_{h}^{R}$ is the cost of replacing hard-type component $h, h=1, \ldots, n_{2} ; c_{h}^{M}$ is the cost of minimally repairing hard-type component $h, \beta_{h}$ is the shape parameter and $\eta_{h}$ is the scale parameter of the Weibull distribution describing times between the failures.

Using Equation (20), the age of a hard-type component can be computed, such that the minimal total cost of repair and replacement is incurred for that component.

Stage 1 provides the output in the form of a vector $\zeta^{*}=\left(\zeta_{1}^{*}, \zeta_{2}^{*}, \ldots, \zeta_{n_{2}}^{*}\right)$, which contains the optimal replacement ages for all hard-type components.

\subsubsection{Stage 2. Obtaining the optimal number of minimal repairs before replacement for soft-type components}

At this stage, the optimal replacement ages of hard-type components, i.e. $\zeta^{*}=$ $\left(\zeta_{1}^{*}, \zeta_{2}^{*}, \ldots, \zeta_{n_{2}}^{*}\right)$, are used as one of the inputs. Because there is no indication of the exact time of failure for a soft-type component (see Figure 7 and Problem Description), a replacement policy for soft-type components cannot be based on the ages of the components at failure, unlike the policy for hard-type components. In this case, one option is to use the number of minimal repairs previously performed as the decision criterion. 
Assuming that soft-type component $s$ is replaced after $m_{s}$ failures, the total expected cost of this soft-type component over the life cycle $\omega$ can be obtained as following:

$$
\begin{aligned}
E\left[C_{\left.s, m_{s}, \tau\right]}^{T}\right] & =\text { Cost of inspections for component } s \\
& + \text { Expected cost of minimal repairs for component } s \\
& + \text { Expected cost of replacements for component } s \\
& + \text { Expected cost of downtime for component } s,
\end{aligned}
$$

where $s=1, \ldots, n_{1}$, and the subscripts indicate that this cost is calculated for each soft-type component $s$ with $m_{s}$ as the number of minimal repairs before replacement, given scheduled inspection period $\tau$. The total expected $\operatorname{cost} E\left[C_{s, m_{s}, \tau}^{T}\right]$ depends on $m_{s}$, since smaller number of repairs indicates more frequent replacements of the component, and vice versa. Downtime penalty cost is incurred from the time the soft component fails until the time it is inspected and fixed. The total downtime of a soft-type component over the cycle $\omega$ is the sum of all the downtime periods incurred by this component's failures. The downtime of a hard-type component is zero, since by assumption, it is inspected and fixed immediately at the time of its failure.

If a soft failure occurs before a hard failure, the failed soft-type component is detected and fixed at the hard failure time. If no hard failure takes place between a soft failure and the next periodic (scheduled) inspection, the soft failure is detected and fixed at the periodic inspection time (see Figure 7). The total expected downtime of soft-type component $s, s=1, \ldots, n_{1}$, is the difference between the length of the life cycle $\omega$ and the component's total expected uptime. Given $l$ scheduled inspections, each with $\operatorname{cost} c_{I}$ and the current number of failures $f_{C}=0$ at the beginning of life cycle $\omega$, Equation (21) can be rewritten as follows:

$$
\begin{aligned}
& E\left[C_{s, m_{s}, \tau}^{T}\right]=\frac{l c_{I}}{n_{1}}+c_{S}^{M} M_{l, s}\left(\tau, t_{s}, \boldsymbol{\theta}, \boldsymbol{\zeta}^{*}, f_{C}, m_{s}\right)+c_{S}^{R} R_{l, s}\left(\tau, t_{s}, \boldsymbol{\theta}, \boldsymbol{\zeta}^{*}, f_{C}, m_{s}\right) S \\
& +c_{s}^{D}\left(\omega-U_{l, s}\left(\tau, t_{s}, \boldsymbol{\theta}, \zeta^{*}, f_{C}, m_{s}\right)\right),
\end{aligned}
$$

where $c_{I}$ is the cost of one periodic inspection, $c_{S}^{M}$ is the cost of minimally repairing component $s, c_{s}^{R}$ is the cost of replacing component $s, c_{s}^{D}$ is the cost of downtime for component $s, t_{j}$ is the initial age of soft-type component $s, l$ is the number of periodic inspections in the cycle $\omega, \boldsymbol{\theta}=$ 
$\left(\theta_{1}, \theta_{2}, \ldots, \theta_{n_{2}}\right)$ is the vector containing initial ages of $n_{2}$ hard-type components, $M_{l, S}\left(\tau, t_{s}, \boldsymbol{\theta}, \zeta^{*}, f_{C}, m_{s}\right), R_{l, S}\left(\tau, t_{s}, \boldsymbol{\theta}, \zeta^{*}, f_{C}, m_{s}\right)$ and $U_{l, S}\left(\tau, t_{s}, \boldsymbol{\theta}, \zeta^{*}, f_{C}, m_{s}\right)$ are the expected numbers of minimal repairs, replacements and expected uptime of soft-type component $s$, respectively. The expected values $M_{l, s}, R_{l, s}$ and $U_{l, s}$ all depend on $\omega, \tau, m_{s}$, the initial age $t_{s}$ of soft-type component $s$ and the vectors $\boldsymbol{\theta}$ and $\zeta^{*}$. The mathematical formulation used for obtaining these values is provided in Appendix B.

The optimal number of minimal repairs before replacement $m_{s, \tau}^{*}$ for each soft-type component $s$ and a given inspection period $\tau$ can be obtained using assumption M.1.10 by first calculating the total expected $\operatorname{cost} E\left[C_{s, m_{s}, \tau}^{T}\right]$ for different values of $m_{s}$ from 0 to an upper bound on the expected number of failures and then comparing the expected costs across all the values of $m_{s}$.

Under assumptions M.1.11 and M.1.12 stated previously, the expected number of failures $E\left[\Phi_{s}\right]$ for a soft-type component $s=1, \ldots, n_{1}$ over $\omega$ can be calculated as following:

$$
E\left[\Phi_{s}\right]=\int_{0}^{\omega} \frac{\beta_{s}}{\eta_{s}}\left(\frac{t_{s}}{\eta_{s}}\right)^{\beta_{s}-1} d t=\left(\frac{\omega}{\eta_{s}}\right)^{\beta_{s}} .
$$

The $\alpha \%$ confidence interval for $E\left[\Phi_{s}\right]$ is then constructed from Poisson distribution as:

$$
\begin{aligned}
& U C L_{s}=\min _{\varphi_{s}}\left\{\sum_{f_{s}=0}^{\varphi_{s}} P\left(\Phi_{s}=f_{s}\right) \geq 1-\left(\frac{1-\alpha}{2}\right)\right\}, \\
& L C L_{s}=\max _{\varphi}\left\{\sum_{f_{s}=0}^{\varphi_{s}} P\left(\Phi_{s}=f_{s}\right) \leq \frac{1-\alpha}{2}\right\},
\end{aligned}
$$

where $P\left(\Phi_{s}=f_{s}\right)=\frac{\left(E\left[f_{s}\right] f_{s} e^{-E\left[f_{s}\right]}\right.}{f_{s} !}$ denotes the probability of observing $f_{s}$ as the expected number of failures over $\omega$.

Then, $m_{s, \tau}^{*}$, which is the optimal number of minimal repairs before replacement for each soft-failure component $j$, can be found from the following marginal optimisation problem:

$$
m_{s, \tau}^{*}=\min _{m_{s}}\left\{E\left[C_{s, m_{s}, \tau}^{T}\right]\right\}, \text { s.t. : } 0 \leq m_{s} \leq U C L_{s} .
$$


At this stage, the optimal number of minimal repairs should be obtained for all soft-type components for different values of $\tau=1,2, \ldots, \omega$. The output of Stage 2 is the matrix $\boldsymbol{m}^{*}=$ $\left[\begin{array}{ccc}m_{1,1}^{*} & \cdots & m_{1, \omega}^{*} \\ \vdots & \ddots & \vdots \\ m_{n_{1}, 1}^{*} & \cdots & m_{n_{1}, \omega}^{*}\end{array}\right]$, in which each column presents the optimal number of minimal repairs for the soft-type components for a value of $\tau$. This matrix is then used as the input for Stage 3 of the method.

In order to obtain $E\left[C_{s, m_{s}, \tau}^{T}\right]$ in Equation (22), it is necessary to first obtain $M_{l, s}, R_{l, s}$ and $U_{l, s}$. However, since the soft failures are hidden, no explicit formula can be given for these expected values. Thus, a simulation model, described below, is developed to numerically obtain the estimates for $M_{l, s}, R_{l, s}$ and $U_{l, s}$.

\subsubsection{1. $\quad$ Simulation model for obtaining the required expected values in Stage 2}

The inputs to the simulation model are the values of $\omega, \tau, t_{s}, m_{s}, \boldsymbol{\theta}, \boldsymbol{\zeta}^{*}, c_{s}^{M}, c_{s}^{R}, c_{s}^{D}$, and $\beta_{s}$ and $\eta_{s}$ for soft-type component $s$ and all hard-type components. The output of the model is $E\left[C_{s, m_{s}, \tau}^{T}\right]$ for the soft-type component $s$. The simulation model generates the times to the first failure for soft-type component $s$ and all hard-type components. Using the Weibull-distributed reliability function $\mathcal{R}_{j}\left(t, \beta_{j}, \eta_{j}\right)$ and a random number $b$ generated from a uniform distribution Uniform $(0 ; 1)$, the next time to failure $\chi_{j}$, given the current component's age $t_{j}$, is generated as follows:

$$
\chi_{j}=\eta_{j}\left(\left(\frac{t_{j}}{\eta_{j}}\right)^{\beta_{j}}-\ln (b)\right)^{1 / \beta_{j}}-t_{j}
$$

The first failure time of the soft-type component is compared with the first failure times of the hard-type components, given the inspection interval $\tau$ and the cycle length $\omega$. If the generated soft failure time is smaller than the next hard failure time, and if the next hard failure time is smaller than the next periodic inspection time, then an opportunistic inspection is occurring at the next hard failure time. At this moment, the soft failure is detected, and if the total number of previous failures is less than $m_{s}$, the component is minimally repaired; otherwise, it is replaced, and its cumulative number of failures is set to zero. If the soft failure's time is not smaller than the next hard failure time, but it is smaller than the periodic inspection 
time, then the soft failure is rectified at the periodic inspection time. If there is a hard failure, the age of the failed hard-type component is compared with its corresponding optimal replacement age given in vector $\boldsymbol{\zeta}^{*}$, and the component is replaced if its age exceeds the optimal age, or it is minimally repaired otherwise. If the component is replaced, its age is set to zero.

At any event's time (i.e. a hard failure's time, a soft failure's time and a periodic inspection's time), the random variables to track the number of minimal repairs, replacements and downtime of the soft-type component $s$ are updated and the simulation clock is advanced to the next event's time. In addition, at an event's time, the time to the periodic inspection, the time to the next failure, and the ages of the surviving components are revised accordingly to capture the passage of time. When the clock reaches the end of life cycle $\omega$, the simulation stops. The averages of the estimates for the random variables obtained from the simulation model executed multiple times provide $M_{l, s}, R_{l, s}$ and $U_{l, s}$.

The total expected costs $E\left[C_{s, m_{s}, \tau}^{T}\right]$ of a soft-type component $s$ from the simulation model obtained for different values of $m_{s}, 0 \leq m_{s} \leq U C L_{s}$, are then compared, and $m_{s}$ resulting in the minimal expected cost is the optimal number of minimal repairs before replacement for component $s$. In Stage 2, using the method described above, the optimal number of minimal repairs is found for all soft-type components. The simulation algorithm for this stage is described in the flowchart given in Appendix C.

\subsubsection{Stage 3. Obtaining the optimal inspection interval for the system}

For Stage 3 of the method, the total expected cost for all soft-type components $E\left[C_{\tau}^{T, S}\right]$ is calculated for the entire system consisting of all soft-type and hard-type components over the system's life cycle for different inspection intervals $\tau=1, \ldots, \omega$. The total expected cost is formulated as following:

$$
\begin{aligned}
E\left[C_{\tau}^{T, S}\right]= & l c_{I}+\sum_{s=1}^{n_{1}}\left(c_{s}^{M} M_{l, s}\left(\tau, t_{s}, \boldsymbol{\theta}, \zeta^{*}, f_{s}, m_{s}^{*}\right)+c_{s}^{R} M_{l, s}\left(\tau, t_{s}, \boldsymbol{\theta}, \boldsymbol{\zeta}^{*}, f_{s}, m_{s}^{*}\right)\right. \\
& \left.+c_{s}^{D}\left(\omega-U_{l, s}\left(\tau, t_{s}, \boldsymbol{\theta}, \boldsymbol{\zeta}^{*}, f_{s}, m_{s}^{*}\right)\right)\right),
\end{aligned}
$$


where superscript $S$ indicates the cost for all soft-type components, given periodic inspection $\tau$. In Equation (27), $M_{l, s}\left(\tau, t_{s}, \boldsymbol{\theta}, \boldsymbol{\zeta}^{*}, f_{s}, m_{s}^{*}\right), R_{l, s}\left(\tau, t_{s}, \boldsymbol{\theta}, \boldsymbol{\zeta}^{*}, f_{s}, m_{s}^{*}\right)$ and $U_{l, s}\left(\tau, t_{s}, \boldsymbol{\theta}, \boldsymbol{\zeta}^{*}, f_{s}, m_{s}^{*}\right)$ represent the expected numbers of minimal repairs, replacements and expected uptime, respectively, for the soft-type component $s$. At this stage, the optimal number of minimal repairs obtained in Stage 2, i.e. $m_{s, \tau}^{*}$, is used for a given $\tau$, as can be seen from Equation (27). With regard to convexity, refer to Appendix A for demonstration.

The optimal inspection interval $\tau^{*}$ is obtained by calculating the total expected cost for different values of $\tau$ and then finding the minimal cost among them:

$$
\tau^{*}=\min _{\tau}\left\{E\left[C_{\tau}^{T, S}\right]\right\}, \text { s. t. : } \tau=1,2, \ldots, \omega .
$$

At Stage $3, M_{l, s}\left(\tau, t_{s}, \boldsymbol{\theta}, \boldsymbol{\zeta}^{*}, f_{s}, m_{s}^{*}\right), R_{l, s}\left(\tau, t_{s}, \boldsymbol{\theta}, \boldsymbol{\zeta}^{*}, f_{s}, m_{s}^{*}\right)$ and $U_{l, s}\left(\tau, t_{s}, \boldsymbol{\theta}, \boldsymbol{\zeta}^{*}, f_{s}, m_{s}^{*}\right)$ have to be obtained for $s=1, \ldots, n_{1}$. At this stage, once again, no explicit formula can be derived for obtaining the required expected values due to the unavailability of the ages of the failed softtype components at inspection times. Thus, a simulation model is developed to obtain $E\left[C_{\tau}^{T, S}\right]$ numerically for $\tau=1, \ldots, \omega$. The recursive equation used for obtaining these expected values mathematically is given in Appendix A.

\subsubsection{1. $\quad$ Simulation model for obtaining the required expected values in Stage 3}

The simulation model in this stage is similar to the simulation model developed in Stage 2. The differences from Stage 2 simulation are: the simulation model here receives $\omega, \tau, t_{s}, m_{s, \tau}^{*}$ for all soft-type components $s=1, \ldots, n_{1}$; the inputs include $\boldsymbol{\theta}, \boldsymbol{\zeta}^{*}, \beta_{j}$ and $\eta_{j}$ for all components $j=1, \ldots, m_{1}+m_{2}$; the output of the model is $E\left[C_{\tau}^{T, S}\right]$ for a given inspection interval $\tau$. In each simulation run for a given $\tau$, the number of soft failures for a soft-type component $s$ is compared with $m_{s, \tau}^{*}$, and if it exceeds $m_{s, \tau}^{*}$, the component is replaced; otherwise, it is minimally repaired. Failures and repairs of all components (soft-type and hard-type) are taken into account in the simulation model for Stage 3 to obtain $E\left[C_{\tau}^{T, S}\right]$. The value of $\tau$ resulting in the minimal $E\left[C_{\tau}^{T, S}\right]$ is the optimal inspection interval $\tau^{*}$, and the optimal number of minimal repairs corresponding to the optimal $\tau^{*}$ (obtained from Stage 2 ) is $m_{s, \tau^{*}}^{*}, s=1, \ldots, n_{1}$.

The overall outputs from the method proposed here are: the vector containing the optimal replacement ages for the hard-type components, i.e. $\zeta^{*}$ (from Stage 1), the optimal periodic 
inspection interval $\tau^{*}$ (from Stage 3), and the optimal number of minimal repairs for each softtype component corresponding to $\tau^{*}$, i.e. $m_{s, \tau^{*}}^{*}, j=1, \ldots, m_{1}$ (from Stages 2 and 3 ). The simulation algorithm for Stage 3 is described in the flowchart in Appendix C.

\subsection{Model 4: Preventive Replacement of Hard-Type Components at Periodic Inspections}

In this section, another model is developed based on an assumption M.2.1 listed previously. The general underlying method of this model's development is similar to the one previously described for the corrective maintenance of hard-type component with opportunistic inspections of soft-type components. The difference comes from the assumption that hard-type components may be preventively replaced at a periodic inspection, based on their ages.

In this model, all hard-type components are checked at periodic inspection times, and their ages are compared to the optimal replacement ages. If a hard-type component's age exceeds the optimal replacement age for this component, it is replaced. Thus, in addition to soft-type components' being checked at periodic and opportunistic inspection times, as prescribed by the model in the previous section, the model presented in the current section also requires inspection of hard-type components at periodic inspection times.

In addition to the cost of periodic inspection $c_{I}$, the model describing preventive replacement of hard-type components with opportunistic inspections of soft-type components also includes the cost of opportunistic inspection $c_{O I}$. Since for Stage 2 of Model 3 the expected cost of failures as given by Equations (21) and (22) is calculated marginally for each soft-type component, it is not influenced by the preventive replacements of hard-type components in Model 4. However, preventive replacements of hard-type components affect the total expected cost of failures at Stage 3 of Model 4. There, the frequency of hard failures and, consequently, that of opportunistic inspections of soft-type components is dependent on potential renewals of hard-type components occurring whenever any one of them is preventively replaced.

The total expected cost for all components $E\left[C_{\tau}^{T, H S}\right]$ is calculated for the entire system consisting of all soft-type and hard-type components over the system's life cycle for different inspection intervals $\tau=1, \ldots, \omega$ as following:

$$
E\left[C_{\tau}^{T, H S}\right]=E\left[C_{\tau}^{T, S}\right]+E\left[C_{\tau}^{T, H}\right]=
$$




$$
\begin{aligned}
& =l c_{I}+\sum_{s=1}^{n_{1}}\left(c_{s}^{M} M_{l, s}\left(\tau, t_{s}, \boldsymbol{\theta}, \boldsymbol{\zeta}^{*}, f_{C}, m_{s}\right)+c_{s}^{R} R_{l, s}\left(\tau, t_{s}, \boldsymbol{\theta}, \boldsymbol{\zeta}^{*}, f_{C}, m_{s}\right)\right. \\
& \left.+c_{S}^{D}\left(\omega-U_{l, s}\left(\tau, t_{s}, \boldsymbol{\theta}, \zeta^{*}, f_{C}, m_{s}\right)\right)\right) \\
& +c_{O I} \sum_{h=1}^{n_{2}}\left(M_{l, h}^{H}\left(\tau, \theta_{h}, \zeta_{h}^{*}\right)+R_{l, h}^{H}\left(\tau, \theta_{h}, \zeta_{h}^{*}\right)\right) \\
& +\sum_{h=1}^{n_{2}}\left(c_{h}^{M} M_{l, h}^{H}\left(\tau, \theta_{h}, \zeta_{h}^{*}\right)+c_{h}^{R}\left(R_{l, h}^{H}\left(\tau, \theta_{h}, \zeta_{h}^{*}\right)+P R_{l, h}^{H}\left(\tau, \theta_{h}, \zeta_{h}^{*}\right)\right)\right)
\end{aligned}
$$

where superscript $H S$ indicates the cost for hard-type and soft-type components, given periodic inspection $\tau$. In Equation (29), $E\left[C_{\tau}^{T, H}\right]$ represents the total expected cost for all hard-type components, $c_{O I}$ is the cost of one opportunistic inspection, $M_{l, h}^{H}\left(\tau, \theta_{h}, \zeta_{h}^{*}\right), R_{l, h}^{H}\left(\tau, \theta_{h}, \zeta_{h}^{*}\right)$, $P R_{l, h}^{H}\left(\tau, \theta_{h}, \zeta_{h}^{*}\right)$ are the expected numbers of minimal repairs, replacements and the expected number of preventive replacements for hard-type component $h, h=1, \ldots, n_{2}$, and the other terms are as previously defined. The convexity of the objective function thus expressed can be argued as shown in Appendix A.

The simulation algorithm provided in Appendix $\mathrm{C}$ is extended for this model to incorporate an additional variable for the number of preventive replacements and an additional renewal point for the ages of hard-type components (flowchart not shown).

\subsection{Numerical Example}

In this section, we apply the models previously described to a numerical example to obtain the optimal maintenance policy and inspection interval for the entire system. The solution procedure is similar for both models, and for the reasons of brevity the two models are considered concurrently in this section.

The system consists of $n_{1}=5$ soft-type and $n_{2}=3$ hard-type components. The input parameters for the failure distributions, the costs of minimal repair, replacement and downtime are given in Table 11. 
Table 11: Parameters of the power law intensity function and costs for different components.

\begin{tabular}{cccclll}
\hline $\begin{array}{c}\text { Component } \\
\text { type }\end{array}$ & $\beta_{j}$ & $\begin{array}{c}\eta_{j} \\
\text { (months) }\end{array}$ & $\begin{array}{l}\text { Minimal } \\
\text { repair } \\
\text { cost, } c_{j}^{M}\end{array}$ & $\begin{array}{l}\text { Replacement } \\
\text { cost, } c_{j}^{R}\end{array}$ & $\begin{array}{l}\text { Downtime } \\
\text { penalty } \\
\text { cost/month, } \\
c_{s}^{D}\end{array}$ \\
\hline \multirow{6}{*}{ Soft } & 1 & 1.3 & 3.5 & $\$ 70$ & $\$ 290$ & $\$ 380$ \\
& 2 & 1.1 & 4.6 & $\$ 45$ & $\$ 150$ & $\$ 250$ \\
& 4 & 2.1 & 6 & $\$ 100$ & $\$ 600$ & $\$ 500$ \\
& 5 & 1.8 & 10 & $\$ 75$ & $\$ 450$ & $\$ 350$ \\
\multirow{2}{*}{ Hard } & 1 & 1.7 & 3.6 & $\$ 125$ & $\$ 540$ & $\$ 400$ \\
& 2 & 1.2 & 3.3 & $\$ 100$ & $\$ 240$ & - \\
\hline
\end{tabular}

The cost of inspections is assumed to be fixed $\left(c_{I}=\$ 50, c_{O I}=\$ 100\right)$. Following the three stages of the method explained above, the proposed models are simulated using 5,000 runs for Stage 2 and 100,000 runs for Stage 3. The choice of the number of simulation runs is based primarily on considerations, such as the error in the calculated mean values and the simulation running time. Given the satisfactory level of error obtained from running the simulation for several thousands of replications, it was decided that taking 5,000 runs is an acceptable choice. The standard error obtained from 100,000 runs for Stage 3 allowed to identify the optimal result which was significantly different from the other suboptimal results.

The parameters were obtained from a case study of programmable infusion pumps, for which the planning horizon was 10 years. For the sake of reducing the computational time for a numerical example, these parameters and the planning horizon were adjusted. Choosing the length of $\omega$ to be 12 months is based on the consideration of the number of possible inspection policies and component combinations, which also amount to the running time of the simulation. At each run, the system was assumed to start with all components in the "as-good-as-new" state. The simulation clock was also updated simultaneously with the system events. The end-point of the simulation was defined at the time $\omega$. Because of this, the simulation had no warm-up period.

Stage 1. The hard-type component parameters from Table 11 are used to calculate the optimal replacement ages using Equation (20). The results are provided in Table 12 below. 
Table 12: Optimal replacement ages for hard-type components.

\begin{tabular}{ll}
\hline $\begin{array}{l}\text { Hard-type } \\
\text { component }\end{array}$ & $\begin{array}{l}\text { Optimal replacement } \\
\text { age (months) }\end{array}$ \\
\hline 1 & 9.4 \\
2 & 10.5 \\
3 & 3.6 \\
\hline
\end{tabular}

Minimal repairs are performed on a hard-type component every time it fails before reaching its corresponding optimal replacement age. Once its age exceeds the optimal replacement age, the hard-type component is replaced.

Thus, the output from Stage 1 is the optimal replacement age vector $\zeta^{*} \approx(9.4,10.5,3.6)$ months for hard-type components $h=1,2,3$.

Stage 2. Having the output $\zeta^{*}$ from Stage 1, the system is then simulated for 5,000 runs considering $\omega=12$ months. Based on the previously-stated assumptions, Equation (23) is used to calculate $E\left[m_{s}\right]$ and Equation (24) is used to obtain $U C L_{s}$. Table 13 below shows the $90 \%$ confidence intervals (CIs) on the expected number of soft failures for each soft-type component.

Table 13: Confidence interval $(90 \%)$ on the expected number of failures.

\begin{tabular}{cccc}
\hline $\begin{array}{c}\text { Soft-type } \\
\text { component }\end{array}$ & $\begin{array}{c}\text { 90\% lower } \\
\text { confidence } \\
\text { limit }\left(L C L_{s}\right)\end{array}$ & $\begin{array}{c}\text { Expected } \\
\text { number of } \\
\text { failures } \\
\left(E\left[m_{s}\right]\right)\end{array}$ & $\begin{array}{c}90 \% \text { upper } \\
\text { confidence } \\
\text { limit }\left(U C L_{S}\right)\end{array}$ \\
\hline 1 & 1 & 4.97 & 9 \\
2 & 0 & 2.87 & 6 \\
3 & 0 & 4.29 & 8 \\
4 & 0 & 1.39 & 4 \\
5 & 2 & 7.74 & 13 \\
\hline
\end{tabular}

As evident from Table 13, the $U C L_{s}$ values for the soft-type components are almost always double the $E\left[m_{s}\right]$ values. The reason for this is because of the $90 \%$ confidence interval. Reducing this confidence interval would bring the $U C L_{s}$ and the $E\left[m_{s}\right]$ values closer, but might decrease the fidelity of the model and the number of probable scenarios it can capture, thus reducing its predictive power. The values of the upper confidence limit $\left(U C L_{S}\right)$ are used for each $s, s=1, \ldots, 5$, in order to calculate $E\left[C_{s, m_{s}, \tau}^{T}\right]$, as given in Equation (22), for $m_{s}=0, \ldots, U C L_{s}$. The optimal number of minimal repairs before replacement $m_{s, \tau}^{*}$ corresponding to $E\left[C_{s, m_{s}{ }^{*}, \tau}^{T}\right]=$ 
$\min \left\{E\left[C_{s, m_{s}, \tau}^{T}\right]\right\}$ is then found by sorting through $E\left[C_{s, m_{s}, \tau}^{T}\right]$ calculated for each soft-type component for inspection intervals of length $\tau=1,2, \ldots, \omega$ over $\omega=12$ months. The results are provided in Table 14.

Table 14: The optimal number of minimal repairs before replacement and the corresponding minimal expected cost for each soft-type component $s=1,2, \ldots, 5$ for inspection intervals of $\tau=1,2, \ldots, 12$ months.

\begin{tabular}{|c|c|c|c|c|c|c|c|c|c|c|}
\hline \multirow{3}{*}{$\begin{array}{c}\text { Inspect. } \\
\text { interval } \\
\tau \\
\text { (months) }\end{array}$} & \multicolumn{10}{|c|}{ Soft-type component } \\
\hline & & 1 & & 2 & & 3 & & 4 & & 5 \\
\hline & $m_{1, \tau}^{*}$ & $\begin{array}{c}E\left[C_{1, m_{s}{ }^{*}, \tau}^{T}\right] \\
(\$)\end{array}$ & $m_{2, \tau}^{*}$ & $\begin{array}{c}E\left[C_{2, m_{s}{ }^{*}, \tau}\right] \\
(\$)\end{array}$ & $m_{3, \tau}^{*}$ & $\begin{array}{c}E\left[C_{3, m_{s}{ }^{*}, \tau}^{T}\right] \\
(\$)\end{array}$ & $m_{4, \tau}^{*}$ & $\begin{array}{c}E\left[C_{4, m_{s}{ }^{*}, \tau}^{T}\right] \\
(\$)\end{array}$ & $m_{5, \tau}^{*}$ & $\begin{array}{c}E\left[C_{5, m_{s}{ }^{*}, \tau}^{T}\right] \\
(\$)\end{array}$ \\
\hline 1 & 8 & 796.70 & 6 & 391.68 & 8 & 875.43 & 4 & 319.71 & 12 & 1443.59 \\
\hline 2 & 9 & 792.38 & 6 & 355.57 & 8 & 861.33 & 4 & 274.59 & 12 & 1436.31 \\
\hline 3 & 9 & 782.33 & 6 & 345.05 & 7 & 864.77 & 4 & 259.39 & 13 & 1427.47 \\
\hline 4 & 8 & 779.61 & 6 & 338.29 & 7 & 853.68 & 4 & 251.40 & 12 & 1435.15 \\
\hline 5 & 7 & 778.07 & 6 & 339.24 & 8 & 849.19 & 4 & 257.03 & 13 & 1418.45 \\
\hline 6 & 8 & 780.07 & 6 & 332.16 & 8 & 857.94 & 4 & 244.16 & 13 & 1431.31 \\
\hline 7 & 8 & 783.21 & 5 & 336.37 & 8 & 847.28 & 4 & 246.64 & 11 & 1424.83 \\
\hline 8 & 9 & 785.17 & 6 & 330.19 & 8 & 860.86 & 4 & 239.91 & 10 & 1430.54 \\
\hline 9 & 9 & 779.03 & 6 & 328.18 & 8 & 853.93 & 4 & 240.70 & 12 & 1433.48 \\
\hline 10 & 9 & 779.34 & 5 & 333.57 & 8 & 853.10 & 4 & 243.70 & 12 & 1422.97 \\
\hline 11 & 9 & 780.22 & 6 & 334.97 & 8 & 853.78 & 4 & 242.20 & 12 & 1419.50 \\
\hline 12 & 9 & 779.20 & 6 & 328.08 & 8 & 852.12 & 4 & 239.79 & 11 & 1419.61 \\
\hline
\end{tabular}

The result of Stage 2 calculations is vector $\boldsymbol{m}^{*}$ of the optimal number of minimal repairs before replacement for periodic inspection interval $\tau=1,2, \ldots, \omega$. Based on these results, softtype component 5 has the highest number of minimal repairs before replacement, averaged over $\tau$, followed by soft-type component 1 , which is closely followed by soft-type component 3 . The characteristic lives $\eta_{s}$ for soft-type components 5 and 1 are very close and much lower than those of the other soft-type components (see Table 11). Therefore, these components are expected to fail more often than the others. For this reason and because of higher replacement costs compared to the minimal repair costs, they incur more minimal repairs before replacement when compared to components with higher characteristic lives. Besides, the cumulative hazard rate, which represents the likelihood of failure given previous history of survivals, calculated for soft-type component 5 over $\tau=1,2, \ldots, 12$ months, is the greatest, whereas that calculated for soft-type component 4 is the smallest of all components. 
Stage 3. Taking $\boldsymbol{m}^{*}$ as one of the inputs, $E\left[C_{\tau}^{T, S}\right]$ is calculated from Equation (27) for both models, and $E\left[C_{\tau}^{T, H S}\right]$ is calculated from Equation (29) for Model 4. The results from 100,000 simulation runs are provided in Table 15 below.

Table 15: Total expected costs for Model 3 and Model 4 for each inspection period $\tau=$ $1,2, \ldots, 12$ months.

\begin{tabular}{ccc}
\hline $\begin{array}{c}\text { Inspection } \\
\text { period, } \tau \\
\text { (months) }\end{array}$ & $\begin{array}{c}\text { Model 3 } \\
E\left[C_{\tau}^{T, S}\right](\$)\end{array}$ & $\begin{array}{c}\text { Model 4 } \\
E\left[C_{\tau}^{T, H S}\right](\$)\end{array}$ \\
\hline 1 & $3886.51 \pm 1.79$ & $15062.64 \pm 4.34$ \\
2 & $3812.84 \pm 1.98$ & $14960.22 \pm 4.32$ \\
3 & $3802.59 \pm 2.06$ & $14842.29 \pm 4.11$ \\
4 & $3767.94 \pm 2.06$ & $14795.45 \pm 4.29$ \\
5 & $3769.80 \pm 2.07$ & $14938.11 \pm 4.21$ \\
6 & $3757.65 \pm 2.11$ & $14845.41 \pm 4.12$ \\
7 & $3758.87 \pm 2.11$ & $14847.89 \pm 4.34$ \\
8 & $3756.90 \pm 2.10$ & $14817.60 \pm 4.20$ \\
9 & $3754.76 \pm 2.09$ & $14755.14 \pm 4.14$ \\
10 & $3764.69 \pm 2.11$ & $14902.67 \pm 4.23$ \\
11 & $3732.46 \pm 2.09$ & $14785.53 \pm 4.30$ \\
12 & $3753.72 \pm 2.13$ & $14786.97 \pm 4.13$ \\
\hline
\end{tabular}

The expected total cost computed at Stage 3 for each of the periodic inspection intervals is composed of the sums of the optimal per-component costs, which, in turn, are based on the optimal number of minimal repairs before replacement for a soft-type component ranging from 0 to $U C L_{s}$. The fact that $U C L_{s}$ values may sometimes be twice higher than the $E\left[m_{s}\right]$ values does not necessarily double the total expected cost for $U C L_{S}$ values compared to $E\left[m_{S}\right]$ values. Depending on the particular parameters and per-component costs, different numbers of minimal repairs before replacement may be optimal. Consequently, the effect of the $E\left[m_{s}\right]$ and the $U C L_{S}$ values on the total expected cost is also different, depending on the particular per-component costs and the latter's ratios, especially the relationship between the cost of replacement and the cost of minimal repair.

Using the total expected costs from Table 15, the graphs of the expected costs of soft-type failures (in \$) vs. different periodic inspection intervals $\tau$, ranging from 1 to 12 months, are plotted separately for Model 3 and Model 4 in Figure 8 and Figure 9 below. 


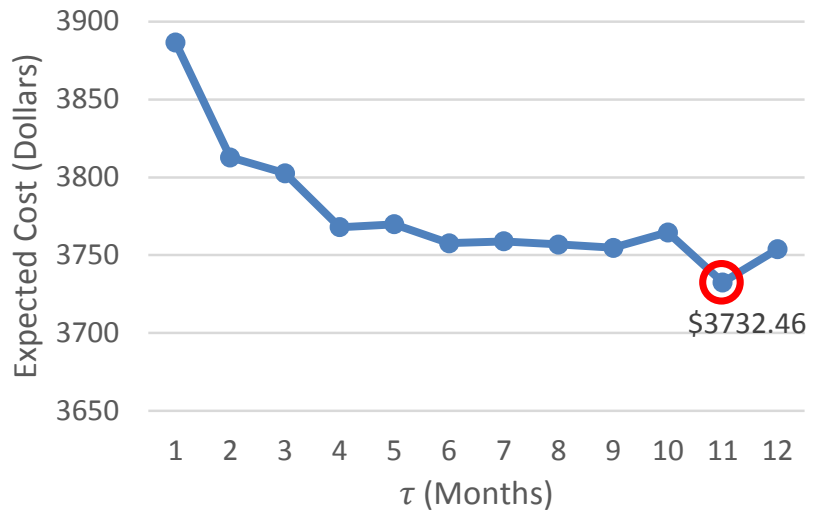

Figure 8: Total expected costs for the case of baseline failure frequency of hard-type components for Model 3.

As can be seen from Figure 8, the optimal inspection period for Model 3 is 11 months, and it results in the total expected soft failure cost of $3732.46 \pm 2.09$ dollars over the cycle of $\omega=$ 12 months. This means that, starting at $i \tau=0$, the system subject to Model 3 assumptions should be optimally inspected at times 11 and 12 months.

Generally, the total expected cost and, hence, the optimal inspection period, depend on the expected number of minimal repairs and replacements and expected downtime for soft-type components, all weighted by their respective costs. In addition, hard-type component parameters describing hard failures also influence the total expected cost by incurring a greater or smaller number of opportunistic inspections. For example, a higher frequency of hard failures results in more opportunistic inspections, which decreases downtime caused by soft failures and increases the number of minimal repairs and replacements, while also increasing the length of the optimal inspection interval. Generally, a shorter periodic inspection interval results in a lower expected number of hard failures until the next periodic inspection, which arises from the orderliness property of the counting process. Depending on the length of the chosen inspection interval, some expected costs decrease, while others increase, changing the total expected cost as a result. For this reason, depending on the given costs of minimal repair, replacement and downtime, as well as their ratios, certain expected values bear greater influence on the total expected cost than others.

The total expected cost calculated at Stage 3 for Model 4 is provided in Figure 9. 


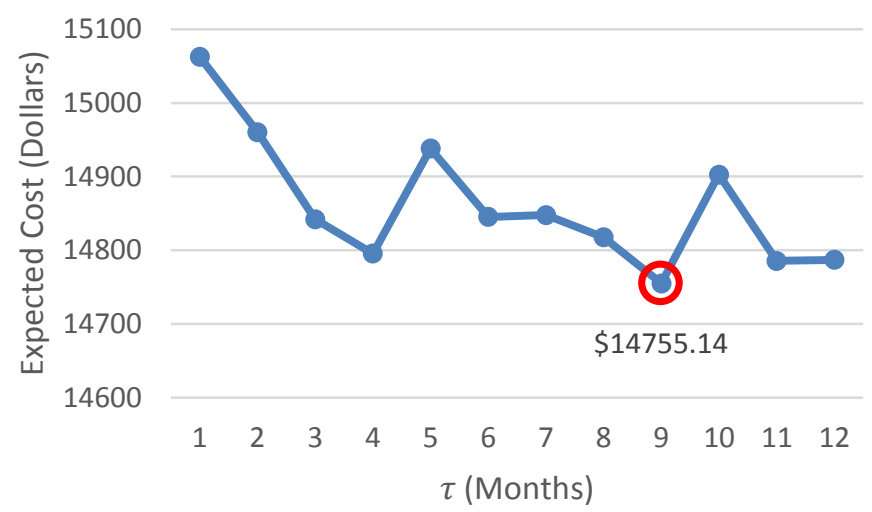

Figure 9: Total expected costs for the case of baseline failure frequency of hard-type components for Model 4.

Figure 9 shows that based on the total expected hard and soft failure cost of $14755.14 \pm$ 4.14 dollars over the cycle of $\omega=12$ months obtained from Model 4, the optimal inspection period is 9 months, and the optimal inspection times are 9 and 12 months. Thus, Model 4 provides a shorter optimal inspection interval than Model 3. This is a result of the fact that preventive replacements of hard-type components cause fewer opportunistic inspections to arise over the life cycle. This results in the lower chance for the soft failures to be detected. Thus, a shorter periodic inspection interval is required to minimise the total expected cost.

The difference in the optimal inspection interval's length between the models is a result of their cost-related features. Model 3 incurs the inspection cost of $\$ 50$ for each periodic inspection, whereas Model 4 incurs $\$ 50$ for each periodic inspection and $\$ 100$ for each opportunistic inspection. This makes opportunistic inspections more costly for Model 4, which results in the optimal periodic inspection interval's being reduced in order to incur fewer opportunistic inspections. This can be ascertained from high-frequency case (see Sensitivity Analysis section below), where Model 3 incurs, on average, 3.5 opportunistic inspections more than Model 4. Also, it is interesting to see that the optimal periodic inspection interval for highfrequency case is the same (12 months) for both models in this case. In the case of the baseline failure frequency, Model 3 incurs, on average, 0.3 opportunistic inspections more than Model 4, but $\tau^{*}$ for Model 3 is 11 months, and that for Model 4 is 9 months. This implies that whenever the number of opportunistic inspections is close for Model 3 and Model 4, Model 4 will tend to have a lower $\tau^{*}$ than Model 3. Conversely, whenever $\tau^{*}$ is close for Model 3 and Model 4, the number of opportunistic inspections will tend to be lower for Model 4, compared to Model 3. 
We further compare the results obtained from using different numbers of minimal repairs before replacement in Stage 3 (see Figure 10(a) for Model 3 and Figure 10(b) for Model 4). The optimal results from the baseline failure frequency of hard-type components for Model 3 (represented by a line with diamond-shaped markers) are compared with the results obtained from assuming that soft-type component replacements take place on $\left(L C L_{s}+1\right)^{\mathrm{st}}$ failure (line with round markers) and the results obtained from assuming that soft-type component replacements take place on $\left(U C L_{S}+1\right)^{\text {st }}$ failure (line with square markers) on Figure 10(a).

As can be seen from the figure, setting the number of minimal repairs before replacement equal to $L C L_{S}$ results in a much higher total expected cost per each tau than both finding the optimal number of minimal repairs before replacement, or setting the number of minimal repairs before replacement equal to $U C L_{s}$. The optimal total expected cost from 100,000 runs is $5050.39 \pm 2.13$ dollars in the case $n_{s, \tau}=L C L_{s}$. Setting $n_{s, \tau}=U C L_{s}$, on the contrary, produces results that are much closer to the results obtained from the optimisation and finding the optimal number of minimal repairs before replacement. The optimal total expected cost from 100,000 runs in the case $n_{s, \tau}=U C L_{s}$ is $3743.77 \pm 2.09$ dollars. The optimal total expected cost from the case of using $m_{s, \tau}^{*}$ is still lower at $3732.46 \pm 2.09$ dollars. These results are not surprising, since $L C L_{s}$ values range from 0 to 2 for the soft-type components (see Table 13), which implies that replacements occur every time soft-type components 2-4 fail, on second failure of soft-type component 1 and on third failure of soft-type component 5 . Since the costs of replacement $c_{S}^{R}$ are much higher than the costs of minimal repair $c_{s}^{M}$ for these components, it follows that the total expected cost of this policy is also much higher compared with the optimal total expected cost for baseline case. Because the optimal numbers of minimal repairs before replacement are close to $U C L_{s}$ for most soft-type components in baseline case, the latter provides the total expected costs close to those prescribed by the case of $m_{s, \tau}=U C L_{s}$. Favouring higher numbers of minimal repairs before replacement in the baseline case is also a result of the much lower costs of minimal repair compared to the costs of replacement.

Similar comparison is presented for Model 4 in Figure 10(b) below. 


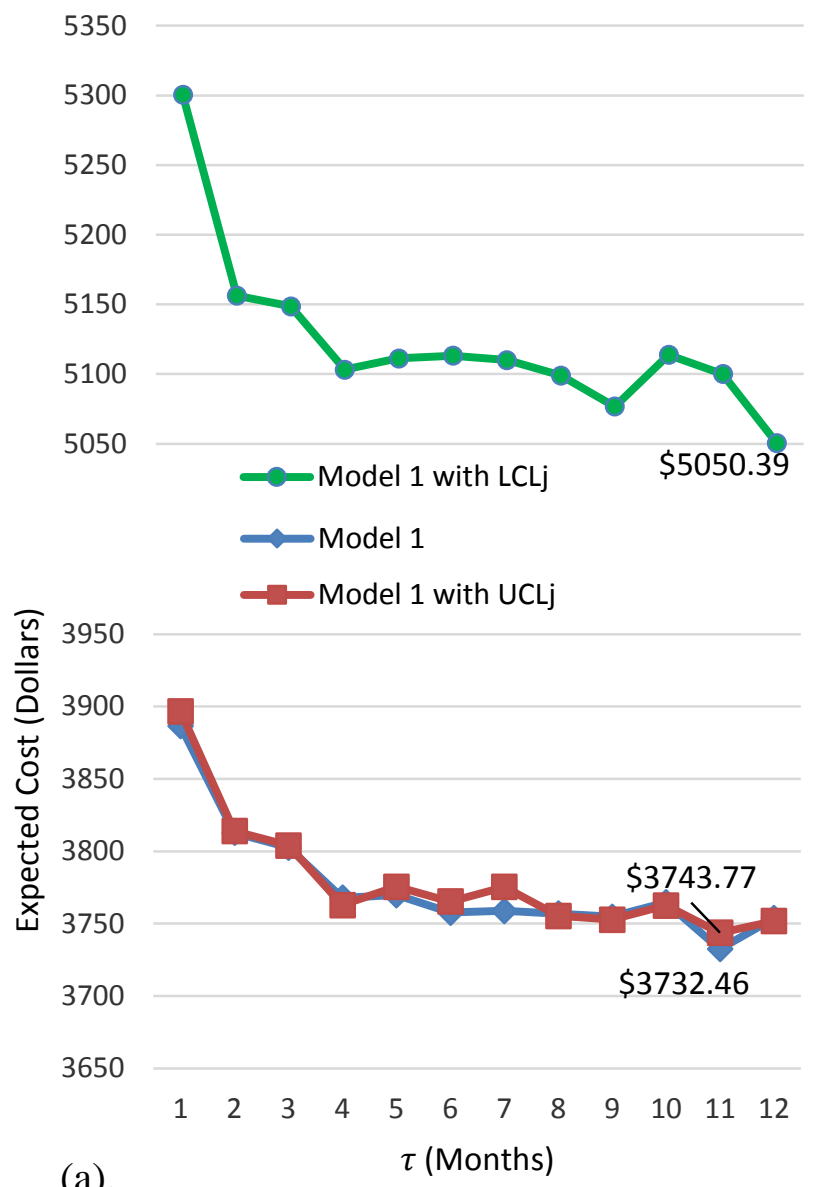

(a).

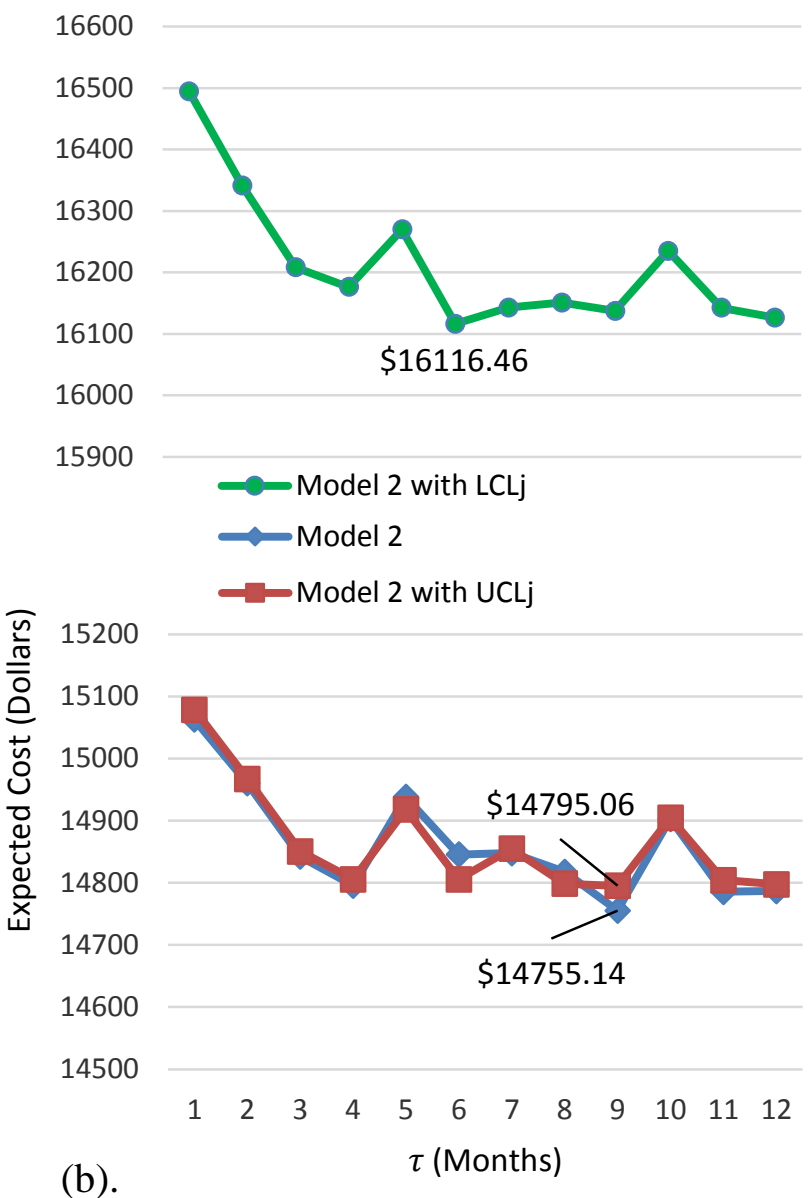

(b).

Figure 10: Total expected costs for baseline Model 3, Model 3 with replacements occurring after $L C L_{s}$ number of component failures, and Model 3 with replacements occurring after $U C L_{s}$ number of component failures (a) and for baseline Model 4, Model 4 with replacements occurring after $L C L_{s}$ number of component failures, and Model 4 with replacements occurring after $U C L_{S}$ number of component failures (b).

As can be seen from Figure 10(b), the optimal total expected cost from 100,000 runs for Model 4 in the case $m_{s, \tau}=L C L_{s}$ is $16116.46 \pm 4.33$ dollars. The optimal total expected cost from 100,000 runs for the case $m_{s, \tau}=U C L_{s}$ is $14795.06 \pm 4.13$ dollars vs. $14755.14 \pm 4.14$ dollars for the baseline Model 4 case. As can be seen, the relationship among different cases for the number of minimal repairs before replacement for Model 4 is similar to that for Model 3. Here too the large difference in the costs of minimal repair and replacement affects the total expected costs for different numbers of minimal repairs before replacement, similarly to Model 3. 


\subsection{Sensitivity Analysis}

Two cases are considered for sensitivity analysis, classified by the frequency of hard-type component failures within the system's life cycle: baseline frequency and high frequency (see Table 16). Distribution parameter $\eta_{h}$ for hard-type components was adjusted to obtain the desired failure frequencies, while parameter $\beta_{h}$ was left as given in Table 11. Generally, the higher the frequency of failure, the lower the optimal replacement age of the corresponding component.

Table 16: Distribution parameters for hard-type components at baseline and high failure frequencies.

\begin{tabular}{l|c|c}
\hline $\begin{array}{l}\text { Hard-type } \\
\text { component }\end{array}$ & $\begin{array}{c}\text { Baseline-freq. failure } \\
\eta_{h} \text { (months) }\end{array}$ & $\begin{array}{c}\text { High-freq. failure } \\
\eta_{h} \text { (months) }\end{array}$ \\
\hline 1 & 3.3 & 1.3 \\
2 & 1.4 & 0.3 \\
3 & 1.3 & 1.2 \\
\hline
\end{tabular}

The sensitivity of the models can be ascertained from the two cases of hard-type failure frequency occurrence. For Model 3, the total expected costs for inspection period of length $\tau=$ $1,2, \ldots, \omega$ over $\omega=12$ months is presented for the two cases in Figure 11. 


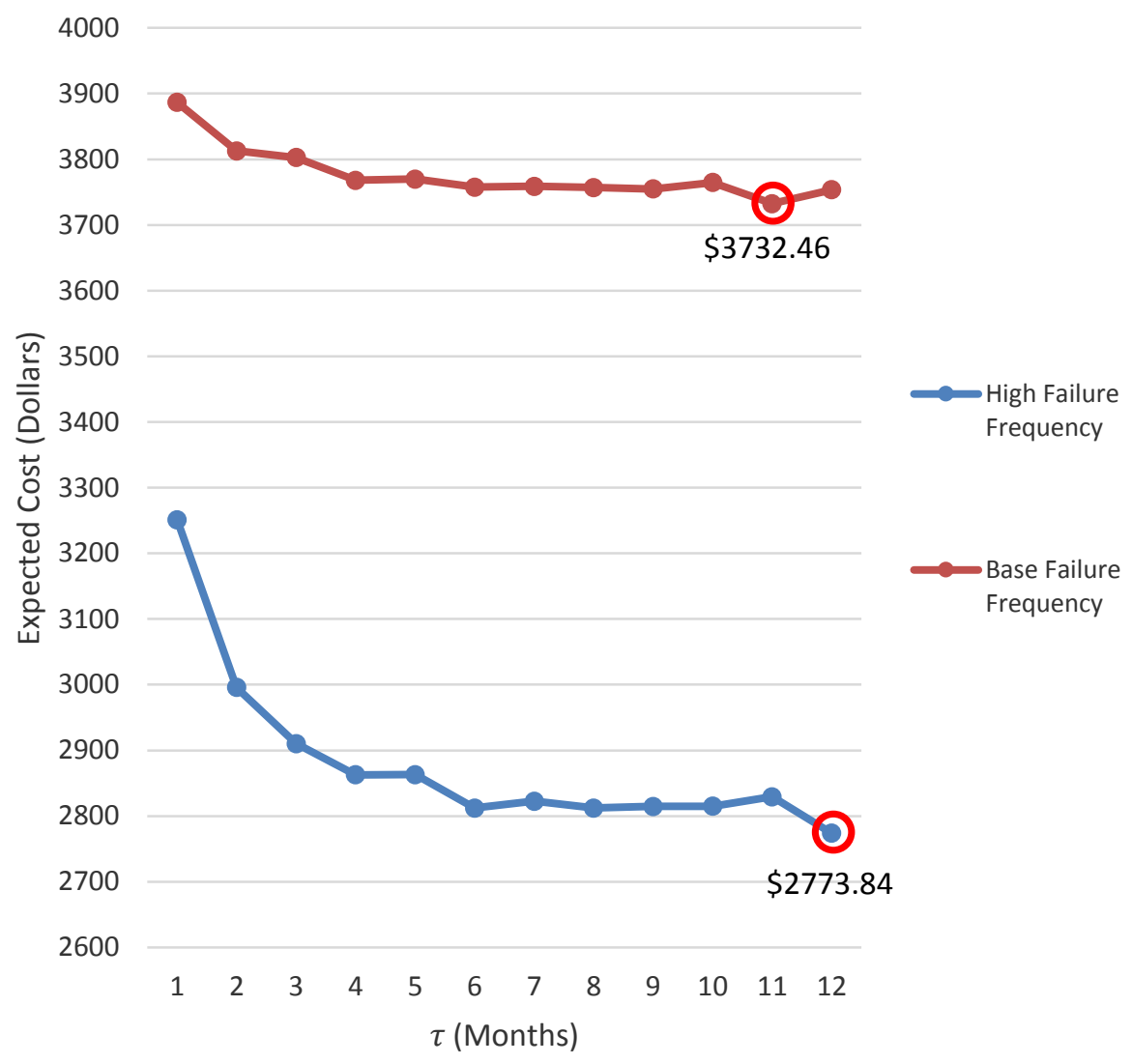

Figure 11: Total expected costs for the cases of baseline and high failure frequencies of hardtype components for Model 3.

Similarly, the total expected costs for inspection period of length $\tau=1,2, \ldots, \omega$ over $\omega=$ 12 months is presented for Model 4 for the two cases in Figure 12. 

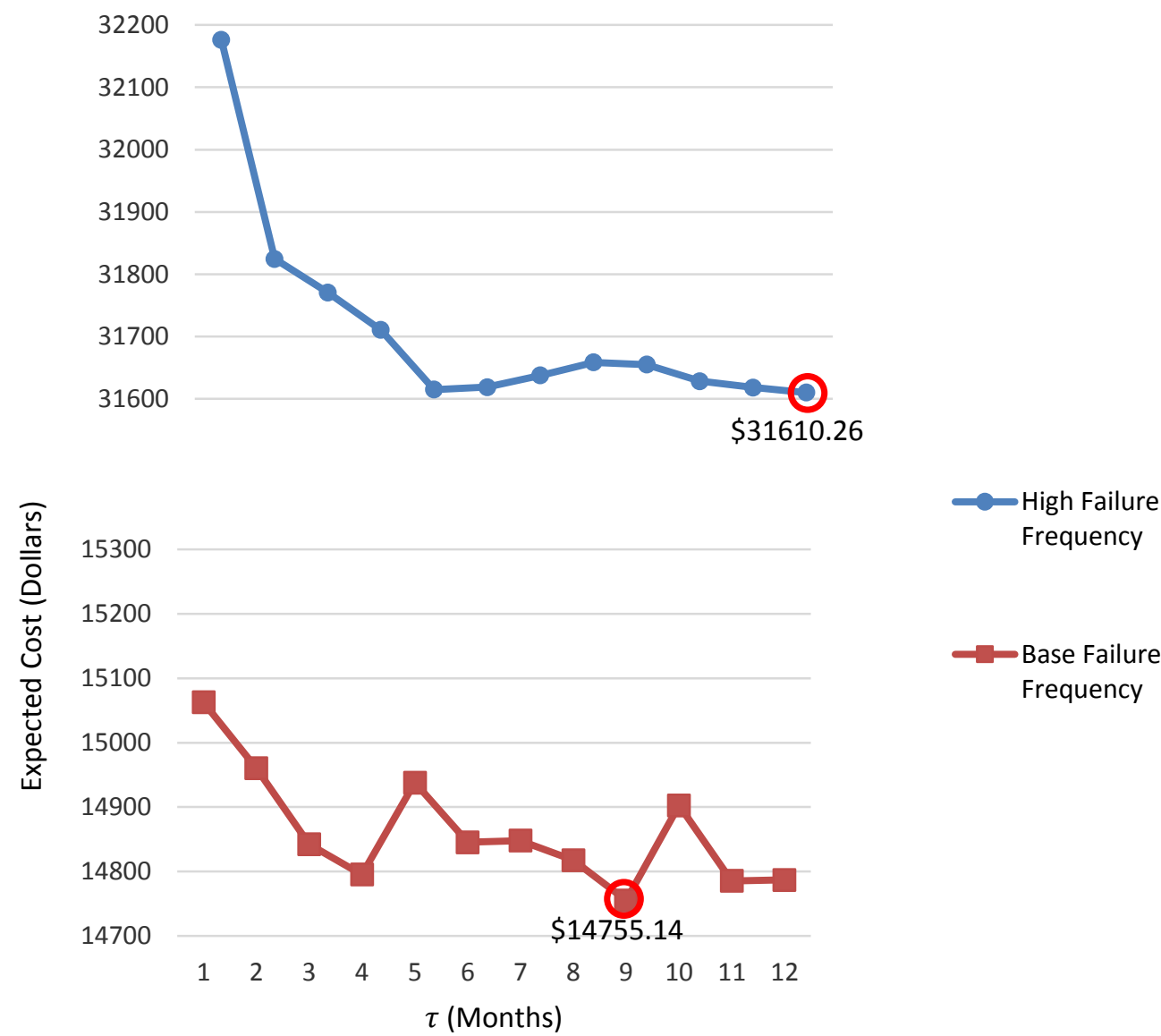

Figure 12: Total expected costs for the cases of baseline and high failure frequencies of hardtype components for Model 4.

As can be seen from Figure 11, the highest total expected cost for Model 3 occurs for the case of baseline hard failure frequency. The fact that baseline hard failure frequency case results in the highest total expected cost can be explained by the higher downtime penalty costs incurred when fewer opportunistic inspections take place due to the increased time between failure of hard-type components. For Model 4, however, the opposite is true: the highest total expected cost for this model occurs for the case of high hard failure frequency (see Figure 12), since in this model the cost of hard failures is also included in the optimisation.

The graphs in Figure 11 and Figure 12 also show that the optimal periodic inspection interval is greater for the case of higher hard failure frequency (12 months for both models) vs. the case of the lower hard failure frequency (11 months for Model 3 and 9 months for Model 4). This 
can be explained by the fact that higher hard failure frequency results in more opportunistic inspections, which decreases the need for scheduled periodic inspections.

In order to estimate how the total expected cost changes with the hard failure frequency, ranges of the total expected cost values were obtained from Model 3 and Model 4, each at high and baseline hard failure frequency, taken for inspection period of length $\tau=1,2, \ldots, \omega$ over $\omega=12$ months. The resulting diagram is shown in Figure 13.

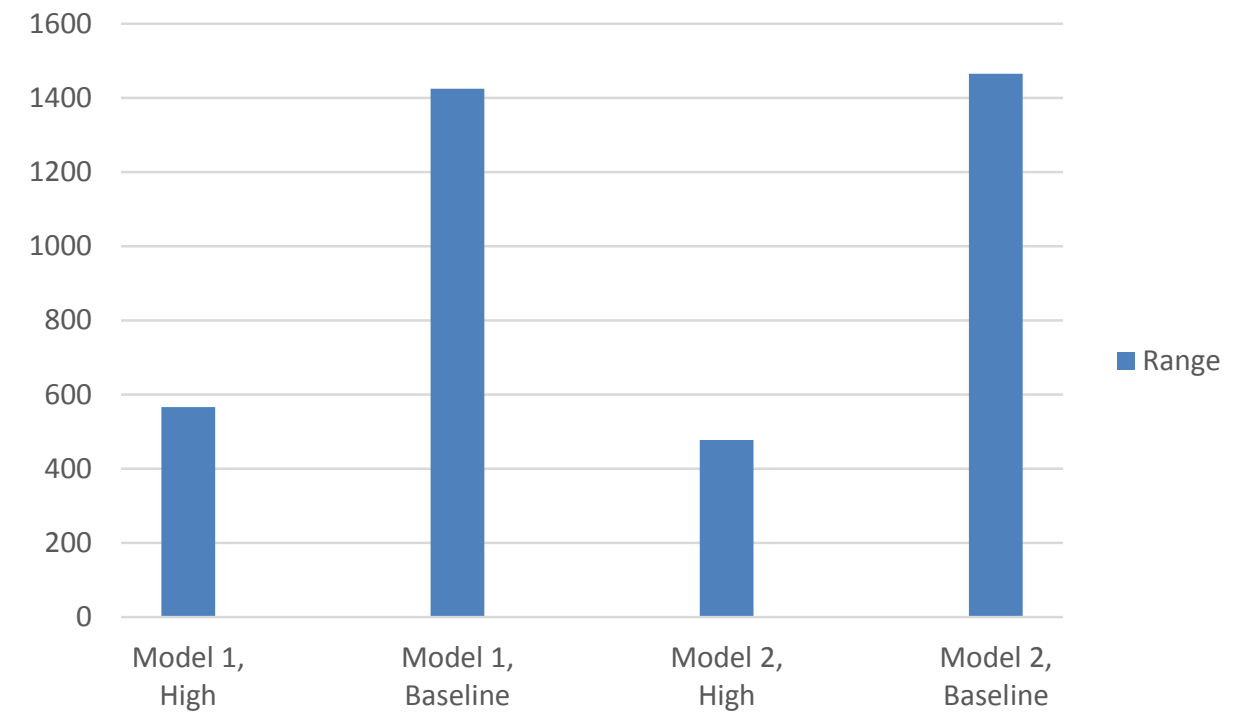

Figure 13: Ranges (in \$) of the total expected cost values for baseline and high hard failure frequencies for each of Model 3 and Model 4.

As can be seen from Figure 13, the range of the total expected costs for both models at the high frequency of hard failures is smaller than that at the baseline frequency. This implies that more benefit can be derived from determining the optimal inspection period length when hardtype components fail rarely within the system's life cycle. Since it is not unreasonable to assume that systems with incorporated protective components, such as medical or power equipment, are not expected to fail too often within their life cycle, the importance of optimisation and the applicability of the proposed models are reinforced.

Figure 13 also shows that the ranges of the total expected costs obtained at both high and baseline hard failure frequencies are close when comparing Model 3 to Model 4. This suggests that the models have similar sensitivity to the fluctuations in the frequency of hard failures. 


\subsection{Conclusion}

The present chapter considers the problem of finding the optimal inspection interval for a system consisting of multiple components with hard-type and soft-type failures, which are all assumed to follow a nonhomogeneous Poisson process (NHPP). When a hard-type component fails, an opportunistic inspection is performed for all soft-type components. Failures of softtype components are assumed to be hidden and revealed only at either scheduled periodic inspections, or unscheduled opportunistic inspections. Thus, the age at failure of soft-type components is not known. At all inspections, a failure is fixed in one of two ways: failed component is either minimally repaired, or it is replaced. Minimal repair does not affect the component's age, while replacement renews the component's age to zero ("as good as new"). The time required to minimally repair or replace any number or combination of components is assumed to be negligible.

The method proposed to find the optimal maintenance actions is divided into 3 stages as following: at the first stage, for each of the hard-type components, the optimal time to replacement resulting in the minimal expected cost for the component per unit time is found; at the second stage, for each soft-type component, the optimal number of minimal repairs before replacement is found, which results in the minimal total expected cost for the component over the system's life cycle; finally, at the third stage, given the optimal maintenance actions for softtype and hard-type components, the optimal periodic inspection interval for the whole system is obtained, which results in the minimal expected cost for the system over its planning horizon.

Two models are considered with numerical example in the present chapter. Model 3 features corrective maintenance of hard-type components with opportunistic inspections of soft-type components. Model 4, in addition to the assumptions pertinent to Model 3, also allows for preventive replacement of hard-type components at periodic inspection times. Using the 3-stage methodology and a numerical example, the optimal inspection interval for Model 4 is found to be shorter than that for Model 3. This may be attributed to the fact that the expected number of opportunistic inspections is lower for Model 4 compared to Model 3, which decreases the opportunities for discovering and correcting soft failures and increases the cost of downtime. Because of this, the optimal inspection interval is shortened to compensate for the lower number 
of expected opportunistic inspections. Furthermore, Model 3 and Model 4 are found to be similarly sensitive to fluctuations in the frequency of hard-type component failures.

As a limitation of the proposed models, it may be seen that the optimisation procedure is not done all in one stage, but rather involves three stages. In other words, the optimal maintenance actions are obtained based on the marginal total cost of each component over the planning horizon, not the cost of the entire system. For finding the optimal inspection interval as well as the optimal maintenance actions in one stage, we require to find the optimal values for several variables at the same time, which makes the optimisation procedure much more difficult, particularly for the systems with a high number of components and longer planning horizon. This is because all various combinations of the possible solutions for the variables are required to be examined.

One extension of the present work which may be considered is a $k$-out-of- $n$ system configuration, where component failures are dependent on each other. For this type of system, we can find the optimal maintenance action for a component, as well as the optimal inspection interval in one stage, based on the total cost of the entire system, because all component are identical. Some possibilities include load-sharing, hidden failures, joint optimisation for optimal inspection interval and either inventory level, or maintenance action.

\section{Acknowledgements}

The author acknowledges the financial support from the Natural Sciences and Engineering Research Council (NSERC) of Canada for this research. 


\section{Non-Periodic Inspection and Maintenance Optimisation of Multicomponent Systems}

The present chapter is based on the following paper:

Babishin V., Hajipour Y., Taghipour S. Non-Periodic Inspection and Maintenance Optimisation of Multicomponent Systems. Computers and Industrial Engineering. Under revision following reviewer's comments.

The present section considers optimisation of inspection and maintenance for multicomponent systems composed of hard-type and soft-type components and $k$-out-of- $n$ components under non-periodic scheduled and opportunistic inspections. Both systems are inspected non-periodically over a finite life cycle. For the $k$-out-of- $n$ system, hidden component failures accumulate and cause system failure when their cumulative number reaches $n-k+1$. For the system with hard-type and soft-type components, hard failures cause system failure, while soft failures are hidden and do not cause immediate failure of the system, but still reduce its reliability. Every system failure allows for an opportunistic inspection of hidden soft-type components in addition to the scheduled inspections.

The objective is to find the optimal non-periodic inspection policy and the optimal maintenance action at each inspection for the entire system. Two types of maintenance are considered: minimal repair and replacement. For hard-type components, the maintenance decision is based on the optimal age before replacement. For hidden failures, because the softtype components' ages at failure are unknown, the maintenance decision is based on the optimal number of minimal repairs before replacement.

Mathematical models are proposed for each of the two system types considered. Because no closed-form expression is available due to the hidden failures, simulation and genetic algorithm (GA) are used to jointly optimise inspection and maintenance policies resulting in the minimum total expected cost. The genetic algorithm can be used with quasi-continuous planning horizon for a greater number of inspection policies and a possibly lower total expected cost. Due to the increasing computational complexity associated with the quasi-continuous 
planning horizon and the number of inspection and maintenance policies to be evaluated, the genetic algorithm presents a promising method of multi-objective optimisation for complex multicomponent systems. Based on the GA's results, some insights are made regarding the system's operation and cost optimisation.

Keywords: Non-periodic inspection, opportunistic inspection, maintenance, hidden soft failure, hard failure, genetic algorithm.

\subsection{Introduction and Background}

The reliability and availability of multicomponent systems drastically affects a vast majority of industries. Failure of certain systems (e.g. nuclear reactor cooling, medical life support systems, etc.) may incur huge costs, present danger to human life and may be associated with catastrophic consequences. Keeping the availability of such systems as high as possible reduces the risks of adverse consequences and excessive costs [110,111, 112]. For this reason, the problem of inspecting and maintaining multicomponent systems is very important.

In the economic sense, for most of the multicomponent systems, repair is more preferable upon failure than replacement, since the latter is usually more labour-intensive, technologically advanced and/or logistically complex. Multicomponent systems generally have higher complexity than unicomponent systems, since the former usually have one or more intercomponent dependencies, such as functional, structural, failure, or economic [1]. Optimal maintenance and economic dependency in multicomponent systems is studied by Dekker et al. [73] and Wang and Pham [72]. Periodic replacement policies for multicomponent systems with stochastic and economic dependencies are investigated by Ozekici [31]. Boland [113] considers time-varying costs of minimal repair under periodic replacement and derives the expected minimal repair cost in an interval in terms of the system's failure rate and the cost function. Series systems with mixed standby components are compared in terms of their cost/benefit ratio, time to failure and long-term availability by Wang and Kuo [13].

Redundant systems with high levels of availability, reliability and robustness are typically represented by a $k$-out-of- $n$ system configuration, where operation of the system is not interrupted upon failure of up to $n-k+1$ components. Multi-engine aircraft, multi-display airplane cockpits, dual-contour automotive brake lines and multiple pumps used for hydraulic 
control are just several examples of $k$-out-of- $n$ systems. A $k$-out-of- $n$ system with perfect component repairs and maintenance equipment subject to imperfect repairs is considered by Zhang and $\mathrm{Wu}$ [29]. Load-sharing $k$-out-of- $n$ systems, where working components' failure rates depend on the number of component failures, are considered by Taghipour [9] and L. Kassaei and Taghipour [10,43]. They minimise the total expected cost and determine the optimal inspection interval for a finite planning horizon.

Another major class of multicomponent systems includes those composed of the two types of components classified by failure: hard-type and soft-type [75]. A hard-type component failure is self-evident and triggers the failure of the entire system; therefore, the time of failure is known for this component type. Examples of hard-type components include: wiring in ignition distributor in automotive electronic ignition, central processing unit in personal computers, fuse and display in infusion pumps, etc. A soft-type component failure does not trigger the immediate failure of the entire system, but the system's reliability is usually reduced as a result of increased risk of malfunction, damage and/or eliminated redundancy. Examples of soft-type components include: liquid-level alarms in infusion pumps and standby-redundant components (batteries, surge-protective equipment, parallel processors). Parts of the system subject to both soft and hard failures are treated as separate soft-type and hard-type components. It can be also noted that components in $k$-out-of- $n$ systems can be treated as soft-type due to the system's capacity to accumulate component failures.

Optimal maintenance of multicomponent systems has been surveyed by Ahmad and Kamaruddin [114], Cho and Parlar [2], Nakagawa and Mizutani and, to a lesser extent, Wang [1]. Optimisation of both inspection and maintenance over a finite-time planning horizon is, however, not considered in these surveys. Sheu et al. [115] determine the optimal number of minimal repairs before replacement in terms of the expected cost per unit time for a twocomponent system under non-homogeneous Poisson pure birth process (NHPPBP) with either minimal repair, or replacement. Sheu et al. [17] consider a bivariate replacement policy for a one-component system, where it is replaced at either the planned age, or after a certain number of minimal repairs, or at the occurrence of catastrophic failure. Chang et al. [116] find the optimal number of minimal repairs before replacement that minimises the long-run expected cost per unit time for a one-component system with a pre-determined threshold as a criterion, exceeding which the system is minimally repaired, or replaced, depending, correspondingly, on 
the probabilities of minor or catastrophic failure. Huynh et al. [117] also consider a periodic condition-based inspection and replacement policy for one-unit system subject to several failure modes due to degradation and shocks.

Periodic inspection policies for complex multicomponent systems have been extensively studied by Taghipour et al. [24], Taghipour and Banjevic [8, 14, 15]. Taghipour and Kassaei [11] consider periodic inspection optimisation for $k$-out-of- $n$ systems.

For almost any system, planning horizon is an indicator of its life expectancy. Fixed and finite planning horizon is used in areas such as pharmacology, medical devices with expiry date, aircraft maintenance (Sriram and Haghani [84]), etc. For example, medications and a vast majority of medical tools have to be replaced once the end of their life cycle has passed. Similarly, aircraft parts usually have to be preventively replaced after a specific number of flight hours.

Systems such as protective devices usually contain components whose failures are hidden. A hidden failure is a failure revealed only at inspection, but not during the normal operation of the system $[16,85]$. The detection of a hidden failure in an integrated system composed of main functional (protected) and safety (protective) units may occur either at inspection, or whenever the protective unit is required to function, but is unavailable because of a failure. Soft failures are similar to hidden failures, but the system is still able to function despite their presence. Single-component systems with hidden failures, probability of failure dependent on the number of previous repairs, and maintenance policy based on both the component's age-at-failure and its number of overhauls are investigated by Sheu et al. [26].

Bjarnason et al. $[19,20]$ consider a joint optimisation model for minimising the total cost of both maintenance and inventory policies for hidden failures in the context of a $k$-out-of- $n$ redundant system. Babishin and Taghipour [86] propose a joint optimisation procedure for minimising the total cost of both periodic and opportunistic inspections and corrective maintenance for a $k$-out-of- $n$ system with hidden failures.

Failure of the system or some of its components can be regarded as an opportunity to check all of the components for damage in addition to the scheduled inspections - hence, whenever such opportunity is taken, inspections performed at that time are called "opportunistic". In the literature, opportunistic maintenance has received an extensive treatment. For example, 
Dagpunar [39] considers opportunistic replacement of a component in a multicomponent system if the failed component's age exceeds a specified control limit. Zhu et al. [40] propose an opportunistic maintenance policy for offshore wind turbines with hard-type and soft-type components and maintenance action for soft-type components dependent on their ages. Cui and $\mathrm{Li}$ [41] use a cumulative damage shock model for a multicomponent system with opportunistic inspections and stochastically-dependent components. Taghipour and Banjevic [14] investigate in one of their models minimal repairs of hard-type and soft-type components over a finite planning horizon for a multicomponent system with opportunistic inspections. Aven and Dekker [42] consider opportunities for preventive replacement for a framework of block and age-based replacement models. Gunn and Diallo [118] use a depth-first shortest path algorithm to search a network tree representation of the opportunistic indirect grouping of periodic preventive replacements problem. Unlike the cases from the literature, in the present case, there is a choice of maintenance action to be taken at each component failure.

Genetic algorithms (GA) have been used in the literature for inspection optimisation of multicomponent systems (Moghaddam and Usher [119], Alrabghi and Tiwari [120], Moura et al. [121], Gen and Cheng [122], Goldberg [123]). Due to the lack of a closed-form solution, joint optimisation is performed using exhaustive search and genetic algorithm with either integer, or quasi-continuous inspection period by Babishin and Taghipour [87]. They provide the optimal joint inspection and maintenance policies, as well as expressions for the expected number of system failures in terms of the cost ratio and component failure intensity for a $k$-outof- $n$ system with hidden failures under both periodic and opportunistic inspections, minimal repair, corrective or preventive replacement. They also derive a criterion for calculating the acceptable number of system failures over the system's life cycle. Bjarnason and Taghipour [21] use the GA to search through a three-dimensional objective function to jointly find the optimal maintenance and $(s, S)$ inventory policies for a $k$-out-of- $n$ system with hidden failures. It should be noted that analysing systems composed of various hard-type and soft-type components, where each component may require a special treatment, is generally much harder than analysing $k$-out-of- $n$ systems, where all components are identical.

Optimal periodic inspection interval and maintenance policies for complex multicomponent systems with hard and hidden soft failures subject to both periodic and opportunistic inspections and age-dependent probabilities of minimal repair or replacement (not subject to optimisation) 
are considered by Taghipour and Banjevic [70]. Unlike the models in [70], in the present chapter, the maintenance decision is optimised in addition to the non-periodic inspection policy.

Non-periodic inspection optimisation has also been covered in the literature. Grall et al. [124] propose non-periodic inspection policy for a one-component deteriorating system. Zhao et al. [32] consider a one-component system subject to replacement under a Gamma deterioration process over infinite planning horizon and develop a model similar to proportional hazards model to optimise the system's non-periodic inspection policy. Su [33] essentially develops a model for optimising non-periodic inspections for a one-component system with a combination of hidden and self-announcing operating modes, since his inspection "period" is a random variable, which renders it non-periodic according to the definitions and terminology adopted in the present chapter. He uses the supplementary variable technique to find the optimal inspection period that maximises profit per unit time.

Multicomponent non-periodic inspections have also been considered in the literature. Hajipour and Taghipour [125] propose a model to find optimal non-periodic inspection policy over a finite planning horizon for multicomponent systems with different maintenance actions performed according to the age-dependent probability. Castanier et al. [34] propose a conditionbased maintenance policy for optimal inspection and replacement of a two-component system under non-periodic inspections, where they essentially develop separate policies for each component, assuming component independence, admitting that extending their approach to larger systems makes the numerical solution intractable. In this regard, it is worth mentioning that Vaurio notes in [36] that it is not generally possible to obtain an analytical solution for the optimal inspection interval even in the simpler case of optimising only for system availability. This explains the interest in and the value of numerical and simulation methods for the analysis of multicomponent systems. Golmakani and Moakedi [37] develop a model for non-periodic inspection optimisation using dynamic programming and branch-and-bound technique, which they use to introduce the $A^{*}$ search algorithm, which attempts to improve on the efficiency of branch-and-bound technique by branching only on the most promising nodes at each step of the search. However, the $A *$ algorithm is at a disadvantage for generating a large number of nodes at each iteration. Some researchers, e.g. Lapa et al. [38], demonstrated the applicability and usefulness of genetic algorithms to optimisation of system availability. In the present chapter, genetic algorithm is used for the purpose of improving efficiency of optimisation calculations. 
To summarise, the present chapter provides a general methodology and two models for finding the optimal joint non-periodic inspection and maintenance policies for complex multicomponent systems with finite planning horizon. In the previous models such as, for example, by Hajipour and Taghipour [125], Taghipour et al. [24, 85], Taghipour and Banjevic $[8,14,70]$, the maintenance action was not optimised, and failed components were minimally repaired, or replaced with age-dependent probabilities. Babishin and Taghipour [87] optimise both maintenance and inspection policies, but only for a $k$-out-of- $n$ system under periodic inspections. Babishin and Taghipour $[71,126]$ use a three-stage optimisation procedure to obtain optimal inspection policy for hard-type components in Stage 1, optimal maintenance in Stage 2 and optimal periodic inspection interval for soft-type components in Stage 3 using the Monte Carlo simulation. The authors are unaware of any previous models which have attempted to optimise both the maintenance action and the inspection policy under non-periodic inspections. In the present chapter, both the maintenance decision and the inspection policy are optimised jointly in one stage. The optimal maintenance policy for soft-type components is based on the number of minimal repairs before replacement for these components, similarly to the approach proposed by Park [30]. The optimal maintenance policy for hard-type components is based on the ages of these components. Both of the proposed models feature corrective maintenance (minimal repair or replacement) of hard-type and soft-type components with opportunistic and scheduled non-periodic inspections of soft-type components. The occurrence of hard failures in the system composed of hard- and soft-type components affects the expected number of soft failures, expected number of minimal repairs, replacements and expected downtime. Therefore, these expected values influence the optimal inspection policy. The components of a $k$-out-of- $n$ system can be regarded as being identical soft-type components, which facilitates the analysis of such systems. Jointly optimising for both inspection and maintenance in one stage for both systems allows to find the optimal inspection and maintenance policies for entire systems rather than marginally only for certain groups of components.

Overall, the safe and reliable operation of different equipment can be facilitated with the help of inspection and maintenance optimisation models. The latter also have strong managerial implications due to the importance of justifying these decisions with both qualitative and quantitative analysis. Using the proposed inspection and maintenance optimisation models, the 
decision-maker(s) gain an opportunity to find the combination of inspection and maintenance decisions that is most likely to result in the greatest cost savings without sacrificing availability or reliability. The hard-to-quantify effects, such as those of opportunistic inspections, can be accounted for by using the joint optimisation models in managerial decision-making process. This is likely to result in cost savings, which are especially significant, if the costs of inspection are high. Thus, it can be seen that optimisation of inspection and maintenance decisions represents a valuable asset for decision-makers.

The present chapter is further structured as following: Section 5.2 states the problem description; Section 5.3 outlines the mathematical model formulation for $k$-out-of- $n$ systems under non-periodic and opportunistic inspections; Section 5.4 contains the mathematical model formulation for the system composed of hard-type and soft-type components under non-periodic inspections and opportunistic inspections of soft-type components; Section 5.5 provides the details of the simulation model for both systems; Section 5.6 provides numerical examples to illustrate the models; finally, Section 5.7 summarises the conclusions.

\subsection{Problem Description}

Consider the problem of inspecting devices consisting of coupled systems, such as surgeprotected personal computers (PCs), infusion pumps with liquid-level alarms, generators or power distributors with reserve power supplies, etc. For such systems, it may not be economically feasible to have periodic inspections - for example, in the case when the optimal inspection period of the protective system does not coincide with the inspection period of the system they are coupled to. In such cases, non-periodic inspections are a good option.

In the present article, two main kinds of multicomponent systems are considered, based on the classification by the types of component failures. The system belonging to the first kind (System 1) consists of identical components in a redundant $k$-out-of- $n$ configuration, with component failures' being hidden and discoverable only upon inspection. System 1 may be represented by several diesel generators providing electrical power to a remote base station (e.g. used in geophysical surveys), which because of crew staffing shortages may require nonperiodic inspection and maintenance optimisation. The system belonging to the second kind (System 2) consists of components belonging to either of the two types: hard type, or hidden soft type. An application of this model includes a MacPherson-type strut assembly found in 
cars, where a shock absorber, a coil spring and a strut-to-mount nut are modelled as hard-type components, and lower and upper spring insulators, dust shield, jounce bumper and spring seat pad are modelled as soft-type components. Both kinds of systems are considered in more detail in subsequent sections.

The present chapter concerns with finding the non-periodic optimal inspection and maintenance policies for two kinds of multicomponent systems described above. The number of components for System 1 is denoted as $n$. The number of soft-type components in System 2 is denoted as $n_{1}$, and the number of hard-type components - as $n_{2}$. For both kinds of systems, the components' failure times are assumed to follow a non-homogeneous Poisson process (NHPP) with the power law intensity function (hazard function) $\lambda_{j}(t)=\frac{\beta_{j}}{\eta_{j}}\left(\frac{t_{j}}{\eta_{j}}\right)^{\beta_{j}-1}$, where $\beta_{j}$ is the shape parameter and $\eta_{j}$ is the scale parameter of the Weibull distribution describing the times between failures of component $j, t_{j}$ is the age of component $j, j=1,2, \ldots, n$ for System 1 and $j=1,2, \ldots, n_{1}+n_{2}$ for System 2, where $s=1,2, \ldots, n_{1}$ is the number of soft-type components and $h=1,2, \ldots, n_{2}$ is the number of hard-type components in the system. For both systems, soft failures are discovered only at inspections. Therefore, the ages at failure of softtype components are unknown.

Inspections are classified as being either scheduled non-periodic, or opportunistic. It is assumed that the system is always inspected at the end of the planning horizon in order to create a renewal point, after which the optimisation procedure can be repeated again. Scheduled nonperiodic (further referred to as simply "non-periodic") inspections occur with the minimal unit of time $\tau$ over a finite planning horizon $\omega$ at times $i \tau, i=1,2, \ldots, l, l \in \mathbb{N}$, where $l=\omega / \tau-1$ if $\omega$ is divisible by $\tau$, and $l=\lfloor\omega / \tau\rfloor$ otherwise. For System 1, opportunistic inspections are incurred whenever $n-k+1$ components fail. For System 2, opportunistic inspections are incurred whenever a hard-type component fails.

A system failure presents an opportunity for inspection of all components in a system. Every failed component is then either minimally repaired, or replaced. In System 1 , the $(n-k+1)^{\text {th }}$ failure presents an opportunity for inspecting the system and rectifying the failed components, which influences the number of minimal repairs, replacements and downtime of the hidden components (see Figure 14). For System 2, hard failures create more opportunities for 
inspecting soft-type components and, consequently, influence the number of minimal repairs, replacements and downtime of the soft-type components (see Figure 15).

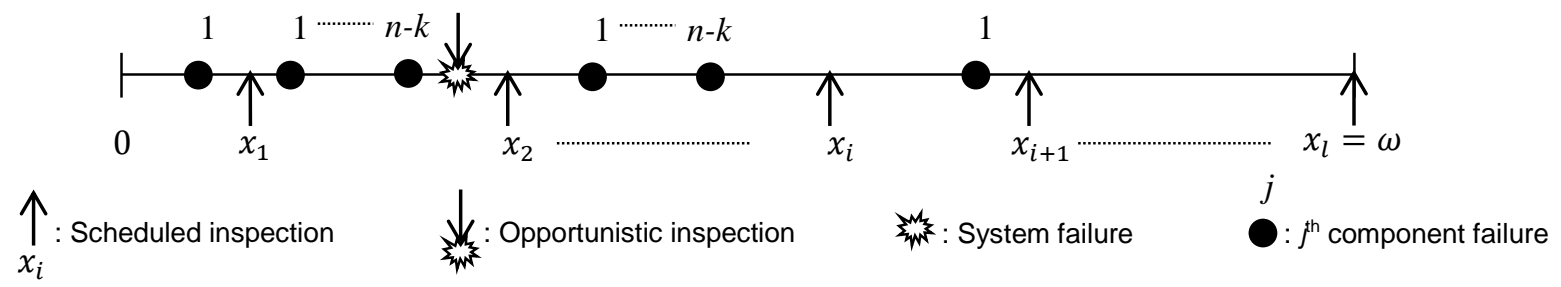

Figure 14: Sample hidden failures, scheduled and opportunistic inspections within one life cycle for System 1.

Figure 14 provides an example of a $k$-out-of- $n$ system, checked at scheduled non-periodic inspections (denoted by $x_{i}$ ) and opportunistically whenever $n-k+1$ components fail. The numbers $1, \ldots, n-k$ above the black circles are denoting the ordinal number of component failures in the system during the period between inspections (and, hence, between failure rectifications). This is used to demonstrate an example of possible occurrence and accumulation of failures within a certain period of time.
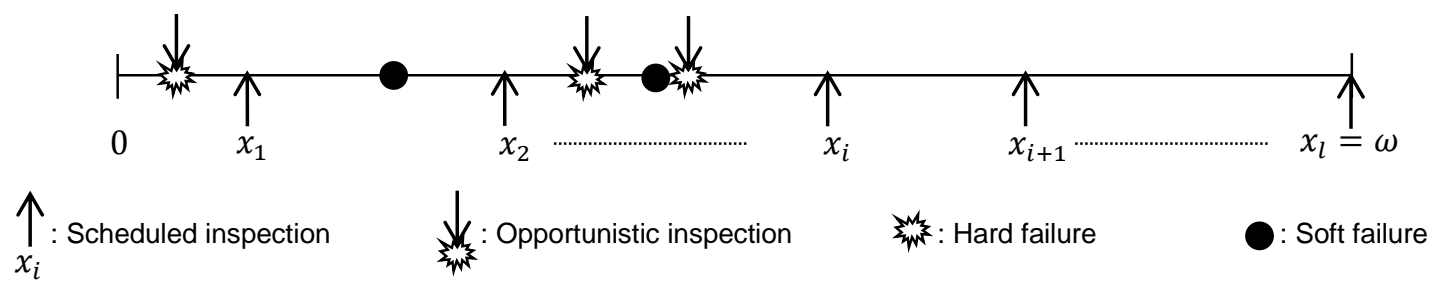

Figure 15: Sample hard and hidden soft failures, scheduled and opportunistic inspections within one life cycle for System 2.

Similarly, Figure 15 shows an example of hard and soft failures along with the scheduled and opportunistic inspections for System 2.

It is assumed that a maintenance action can be classified as either a minimal repair, or a corrective replacement (further referred to as simply "replacement"). A minimal repair restores the component's functionality to the state it was in just preceding the component's failure, thus leaving the component's age unaffected. A corrective replacement decreases the failed component's age to 0 ("as-good-as-new" state). Minimal repairs and replacements can occur both at the time of scheduled, or opportunistic inspections. 
Both maintenance and inspection are assumed to have negligible duration and are perfect. Once the end of the planning horizon (i.e. time $\omega$ ) is reached, a new optimal non-periodic inspection and maintenance policies can be found repeating the outlined procedure. In the case of System 2, current ages of hard-type components can be taken into account when planning for the system life cycle.

The total cost of system maintenance and inspection is a metric used almost universally in different areas of industry for a large variety of systems. It is a convenient measure of the optimality of a system, since the latter's reliability and availability are connected through a range of costs, such as inspection and maintenance costs, component and system downtime penalties, etc. For this reason, the objective function in the case of both System 1 and System 2 is formulated in terms of the total cost of inspection and maintenance policies.

A closed deterministic formulation requires knowledge of all the relevant parameters of the system with certainty. In the present case, however, this condition is not satisfied, since the hidden soft-type components' ages at failure are unknown. In order to describe the expected values of the system, a recursive formulation can be developed. However, the recursive formula does not have a closed-form solution, as it incorporates multidimensional integrals requiring discretisation, which makes the computations cumbersome. Because of this, the present analysis is based on the results obtained from stochastic programming and numerical Monte-Carlo simulations, as well as on the use of the genetic algorithm (GA).

To summarise, the objective of the current work is to determine the optimal non-periodic inspection policy $x^{*}$ and the optimal maintenance policy $m^{*}$ for System 1 and $m_{s}^{*}$ for System 2. The optimal inspection and maintenance policies are achieved by minimising the total expected cost for the entire system over the planning horizon $\omega$.

\subsection{Model 5: Joint Optimisation of Non-Periodic Inspection and Corrective Maintenance of $\boldsymbol{k}$-out-of- $\boldsymbol{n}$ System with Opportunistic Inspections}

In this section, a model is proposed for a $k$-out-of- $n$ system that can be non-periodically checked at potential times $i \tau$, where $\tau$ is the minimal time unit. If the choice of $\tau$ is sufficiently small relative to the planning horizon $\omega$, then the variable for the inspection interval length may be considered as quasi-continuous. Thus, finding the optimal non-periodic inspection policy generally amounts to quasi-continuous optimisation. At the same time, maintenance 
optimisation is done for the discrete-valued number of minimal repairs before replacement. Thus, overall, joint quasi-continuous and discrete optimisation is performed in order to obtain the optimal joint inspection and maintenance policies.

Maintenance optimisation is concerned with identifying the best maintenance action for a particular system setting. In the present chapter, at each inspection point, the decision has to be made whether to minimally repair, or replace the failed component(s). Inspection optimisation then provides the best points in time at which these maintenance actions have to be taken in order to incur the lowest cost. Since failures are stochastic in nature, the total expected cost is used for optimality computations.

The scheduled non-periodic inspection policy $\boldsymbol{x}^{a}=\left(x_{1}, x_{2}, \ldots, x_{l}\right)$, where $a$ refers to the inspection policy index, can be encoded as a binary sequence of ' 1 's and ' 0 's, where each ' 1 ' corresponds to a scheduled inspection and ' 0 ' corresponds to the lack thereof. Taking the number of digits of $\boldsymbol{x}^{a}$ to be $l$, each digit then corresponds to time $i \tau$. This binary representation lends itself naturally to the encoded "genome" strings used in the genetic algorithms, which makes it particularly convenient and effective for the purposes of inspection optimisation using the latter. The total number of possible distinct scheduled inspection policies is then $2^{l-1}$, since there is always 1 inspection scheduled to occur at time $\omega$. Hence, enumerating $\boldsymbol{x}^{a}, a=$ $1,2, \ldots, 2^{l-1}$.

The components' failures are hidden, making their ages at failure unknown. For this reason, maintenance decisions cannot be based on the age. Instead, the number of minimal repairs is counted for each component, and the decision of whether to minimally repair, or replace a failed component is based on the number of minimal repairs before replacement. Since all of the components in a $k$-out-of- $n$ system are identical by definition, only one optimal number of minimal repairs before replacement has to be found for a given system. The number of minimal repairs before replacement depends on the expected number of component failures, since there is a statistical uncertainty associated with the latter. In obtaining the expected number of component's failures $E[\Phi]$, the following two assumptions are made: (1) each inspection detects failure immediately, and (2) maintenance does not affect the ages of failed components (i.e. failed components are always minimally repaired). Based on these, $E[\Phi]$ is obtained as following: 


$$
E[\Phi]=\int_{0}^{\omega} \frac{\beta}{\eta}\left(\frac{t}{\eta}\right)^{\beta-1} d t=\left(\frac{\omega}{\eta}\right)^{\beta}
$$

The actual number of failures is expected to fall within a $(100 * \alpha) \%$ confidence interval, with the upper confidence limit $U C L$ given as:

$$
U C L=\left(\min _{\varphi} \mid \sum_{f=0}^{\varphi} P(\Phi=f) \geq 1-\left(\frac{1-\alpha}{2}\right)\right)
$$

where $U C L$ is the upper confidence limit for a component, $m$ is the number of minimal repairs before replacement, and the probability of observing $\varphi$ failures over planning horizon $\omega$ is given by $P(\Phi=\varphi)=\frac{(E[\varphi])^{\varphi} e^{-E[\varphi]}}{\varphi !}$ for each soft-type component.

The total expected cost $E\left[C_{x^{a}, m}^{T, k, n}\right]$ is formulated as following:

$$
\begin{aligned}
E\left[C_{x^{a}, m}^{T, k, n}\right] & =l c^{I}+F(\omega, \boldsymbol{t}, k, n) c^{S D}+\sum_{j=1}^{n}\left(c^{M} M_{x^{a}, m, j}\left(\omega, t_{j}, k, n\right)\right. \\
& +c^{R} R_{x^{a}, m, j}\left(\omega, t_{j}, k, n\right)+c^{D}\left(\omega-U_{x^{a}, m, j}\left(\omega, t_{j}, k, n\right)\right),
\end{aligned}
$$

where $l$ is the number of scheduled inspections in the cycle $\omega, c^{I}$ is the cost of one scheduled inspection, $\boldsymbol{t}=\left(t_{1}, t_{2}, \ldots, t_{n}\right)$ is the vector containing the components' initial ages, $F(\omega, \boldsymbol{t}, k, n)$ is the expected number of system's failures, $c^{S D}$ is the system downtime penalty cost, $c^{M}$ is the cost of component minimal repair, $c^{R}$ is the cost of component's corrective replacement, $c^{D}$ is the cost of component downtime per unit time, $t_{j}$ is the initial age of component $j$, $M_{x^{a}, m, j}\left(\omega, t_{j}, k, n\right), R_{x^{a}, m, j}\left(\omega, t_{j}, k, n\right)$ and $U_{x^{a}, m, j}\left(\omega, t_{j}, k, n\right)$ are the expected numbers of minimal repairs, replacements and expected uptime of component $j$, respectively. In the proposed formulation, the expected values are generally obtained using the variables indicated inside the brackets as parameters. To ascertain the convexity of the total expected cost function, refer to Appendix A.

The optimal joint inspection and maintenance policies are defined by the optimal inspection policy $\boldsymbol{x}^{*}$ and the optimal number of minimal repairs before replacement $m^{*}$, correspondingly. Using the calculations for the combinations of possible inspection and maintenance policies 
$\left(\boldsymbol{x}^{a}, m\right)$, the optimal joint inspection policy $\left(\boldsymbol{x}^{*}, m^{*}\right)$ can be obtained from searching for the smallest total expected cost as following:

$$
\begin{aligned}
& \left(\boldsymbol{x}^{*}, m^{*}\right)=\min _{\boldsymbol{x}^{a}, m}\left\{E\left[C_{\boldsymbol{x}^{a}, m}^{T, k, n}\right]\right\}, \\
& \text { s. t. : } 0 \leq m \leq U C L, \\
& \qquad x_{i}=\left\{\begin{array}{l}
1, \text { if there is an inspection at time } i \tau \\
0, \text { if there is no inspection at time } i \tau
\end{array}, i=1,2, \ldots, l .\right.
\end{aligned}
$$

The expected values required for the calculation of $E\left[C_{x^{a}, m}^{T, k, n}\right]$ are, however, unavailable for a system with hidden failures, since the ages at failure are unknown and there is no explicit formulation for them. This obstacle is overcome by using the simulation procedures described in Section 5.5.

\subsection{Model 6: non-periodic inspection and corrective maintenance of hard-type and soft-type components with opportunistic inspection of soft-type components}

This section describes the methodology for finding the optimal maintenance actions after failures and the optimal inspection policy for System 2, taking into account the fact that soft failures are hidden and the ages of the soft-type components at the time of failure are unknown.

Due to the different failure characteristics, hard-type and soft-type components are analysed separately. The failure times of hard-type components are known, since the system stops operating immediately whenever a hard failure occurs. The goal is to find the optimal ages at which the hard-type components should be replaced, resulting in the lowest total expected cost for the system. In order to achieve this, the domain of possible replacement ages from which to choose the optimal ones has to be defined for each hard-type component. The replacement ages are represented by vector $\zeta=\left(\zeta_{1}, \zeta_{2}, \ldots, \zeta_{n_{2}}\right)$, consisting of replacement ages for each hard-type component $h=1,2, \ldots, n_{2}$. From the system life cycle's perspective, it is impractical to make the hard-type component's replacement age longer than the life span of the entire system, as represented by its planning horizon. It is assumed that an overhaul or similar renewal event is to take place at the end of the system's planning horizon, at which point those hard-type components which have not been maintained over the system's operation will be replaced. Thus, the replacement ages for hard-type components can be assumed to be bounded by 0 from the 
bottom and a multiple of the system's life cycle length at the top, for example: $0<\zeta_{h} \leq 1.5 \omega$, $h=1,2, \ldots, n_{2}$. The optimal replacement ages for all hard-type components are represented by vector $\zeta^{*}=\left(\zeta_{1}^{*}, \zeta_{2}^{*}, \ldots, \zeta_{n_{2}}^{*}\right)$ consisting of the optimal replacement ages for each hard-type component $h=1,2, \ldots, n_{2}$.

Unlike those of hard-type components, the failure times of soft-type components are unknown, which makes it impossible to base the optimisation procedure on the ages of softtype components. Instead, the number of minimal repairs before replacement may be used as the maintenance criterion. Similarly to System 1 and using the same assumptions, the expected number of component failures $E\left[\Phi_{S}\right]$ for System 2 was obtained as following:

$$
E\left[\Phi_{s}\right]=\int_{0}^{\omega} \frac{\beta_{s}}{\eta_{s}}\left(\frac{t_{s}}{\eta_{s}}\right)^{\beta_{s}-1} d t=\left(\frac{\omega}{\eta_{s}}\right)^{\beta_{s}} .
$$

The actual number of failures, however, may differ among the components, owing to the stochastic nature of component failures. Hard failures are assumed to be rectified immediately upon failure. Soft failures are rectified at the earlier of either a scheduled inspection, or hard failure, which presents an opportunity for inspection and fixing of the system (hence the name "opportunistic inspection"). The general estimate on the upper bound of the number of minimal repairs before replacement can then be obtained from constructing a confidence interval of width $\alpha$ from Poisson distribution for $E\left[\Phi_{S}\right]$ as following:

$$
U C L_{s}=\left(\min _{\varphi_{s}} \mid \sum_{f_{s}=0}^{\varphi_{s}} P\left(\Phi_{s}=f_{s}\right) \geq 1-\left(\frac{1-\alpha}{2}\right)\right),
$$

where $U C L_{s}$ is the upper confidence limit for soft-type component $s$, and the rest of terms are as previously defined.

It is assumed that the number of minimal repairs before replacement $m_{s}$ for soft-type component $s, s=1, \ldots, n_{1}$ does not exceed the upper confidence limit $U C L_{s}$ on the expected number of component failures and may take on any value between 0 and $U C L_{s}$, inclusively. Thus, different cases are covered, ranging from replacement on every failure to replacement on $\left(U C L_{s}+1\right)^{\text {st }}$ failure. Furthermore, the chosen value of $m_{s}$ serves as the criterion for making a maintenance decision. Component $s$ is minimally repaired at each inspection until the number 
of its failures reaches $m_{s}$. It is then replaced on $\left(m_{s}+1\right)^{\text {st }}$ failure. The optimal number of minimal repairs before replacement $m_{s}^{*}$ results in the lowest total expected $\operatorname{cost} E\left[C_{x^{a}, m_{s}, \zeta_{h}}^{T, H S}\right]$ for the entire system.

It should be noted, that unlike in preventive replacement models for mixed systems composed of hard- and soft-type components encountered in Babishin and Taghipour [126], corrective replacement models may exclude the costs of hard-type components from the optimisation. This is because the hard-type components are replaced at the optimal replacement ages if they fail, and not at the scheduled inspection times when they are still operational. This makes the optimal non-periodic inspection independent of the costs of hard failures.

In order to obtain the lowest expected cost, all combinations of non-periodic inspection schedules, the numbers of minimal repairs before replacement, and various ages as threshold for replacement have to be considered for all soft-type components and hard-type components, respectively. The expected costs thus calculated can then be searched for the lowest value. However, the size of the search space is very large in this case. For this reason, this problem, albeit in the context of periodic inspections, has been previously broken down into several stages for maintenance and inspection optimisation for all hard-type components in Stage 1, marginal optimisation of the maintenance decision for each soft-type component in Stage 2 and optimisation of the inspection period for the entire system in Stage 3 [71, 126]. Monte-Carlo simulation was used for marginal multi-stage optimisation. In the present chapter, global system-level optimisation is performed, which requires simultaneous optimisation of all variables, including the optimal ages to replace hard-type components, the optimal number of minimal repairs before replacement for soft-type components and the optimal inspection scheme. This results in a dramatic increase of the search space. The latter is greatly reduced by means of the genetic algorithm. This allows optimising for both inspection and maintenance jointly in one stage. The total expected $\operatorname{cost} E\left[C_{\boldsymbol{x}^{a}, m_{s}, \zeta_{h}}^{T, H S}\right]$ is calculated as following:

$$
\begin{aligned}
E\left[C_{\boldsymbol{x}^{a}, m_{s}, \zeta_{h}}^{T, H S}\right] & =l c^{I}+\sum_{s=1}^{n_{1}}\left(c_{s}^{M} M_{\boldsymbol{x}^{a}, m_{s}, s}\left(\omega, t_{s}, \boldsymbol{\theta}, f_{s}, \zeta\right)\right. \\
& +c_{s}^{R} R_{\boldsymbol{x}^{a}, m_{s}, s}\left(\omega, t_{s}, \boldsymbol{\theta}, f_{s}, \zeta\right)+c_{s}^{D}\left(\omega-U_{\boldsymbol{x}^{a}, m_{s}, s}\left(\omega, t_{s}, \boldsymbol{\theta}, f_{s}, \zeta\right)\right)
\end{aligned}
$$


where superscript $H S$ indicates the cost for System 2 consisting of hard-type and soft-type components, $\boldsymbol{\theta}=\left(\theta_{1}, \theta_{2}, \ldots, \theta_{h}, \ldots, \theta_{n_{2}}\right)$ is the vector containing the initial ages of hard-type component $h, h=1,2, \ldots, n_{2}, \zeta=\left(\zeta_{1}, \zeta_{1}, \ldots, \zeta_{n_{2}}\right)$ is the vector containing the replacement ages of hard-type components, $t_{s}$ is the initial age of soft-type component $s, c_{s}^{M}$ is the cost of minimally repairing component $s, c_{s}^{R}$ is the cost of replacing component $s, c_{s}^{D}$ is the cost of downtime for component $s, \quad M_{\boldsymbol{x}^{a}, m_{s}, s}\left(\omega, t_{s}, \boldsymbol{\theta}, f_{s}, \zeta\right), \quad R_{\boldsymbol{x}^{a}, m_{s}, s}\left(\omega, t_{s}, \boldsymbol{\theta}, f_{s}, \zeta\right) \quad$ and $U_{\boldsymbol{x}^{a}, m_{s}, s}\left(\omega, t_{s}, \boldsymbol{\theta}, f_{s}, \zeta\right)$ represent the expected numbers of minimal repairs, replacements and expected uptime, respectively, for the soft-type component $s, s=1,2, \ldots, n_{1}$. Appendix A contains a general argument in favour and a demonstration of the convexity of the total expected cost objective function.

Using the calculations for the combinations of possible inspection and maintenance policies $\left(\boldsymbol{x}^{a}, m_{s}, \zeta_{h}\right)$, the optimal joint inspection and maintenance policy $\left(\boldsymbol{x}^{*}, m_{s}^{*}, \zeta^{*}\right)$ can be obtained from searching for the smallest total expected cost as following:

$$
\begin{gathered}
\left(\boldsymbol{x}^{*}, m_{s}^{*}, \zeta^{*}\right)=\min _{\boldsymbol{x}^{a}, m_{s}, \zeta_{h}}\left\{E\left[C_{\boldsymbol{x}^{a}, m_{s}, \zeta_{h}}^{T, H S}\right]\right\}, \\
\text { s.t. : } 0 \leq m_{s} \leq U C L_{s}, s=1,2, \ldots, n_{1}, \\
\quad 0 \leq \theta_{h} \leq \zeta_{h}, h=1,2, \ldots, n_{2}, \\
x_{i}^{a}=\left\{\begin{array}{l}
1, \text { if there is an inspection at time } i \tau \\
0, \text { if there is no inspection at time } i \tau
\end{array}, i=1,2, \ldots, l, a=1,2, \ldots, 2^{l-1} .\right.
\end{gathered}
$$

The following section outlines the general simulation procedure used for optimisation.

\subsection{Simulation Model}

Simulation procedure is similar for both systems, but differs in some details as a result of the difference in the types of system's components.

\subsubsection{Simulation model for $k$-out-of-n system (System 1)}

The $k$-out-of- $n$ system takes as inputs the values of $x_{i}^{a}, m, k, n, \omega, \tau, t_{j}, \beta, \eta, c^{M}, c^{C R}, c^{P R}$, $c^{D}, c^{I}$ and $c^{S D}$. These are then used to generate the time-to-failure for each component $j, j=$ $1,2, \ldots, n$ using each component's current age $t_{j}$, a random number $b$ generated from a uniform 
distribution Uniform $(0 ; 1)$ and assuming the Weibull-distributed reliability function $\mathcal{R}_{j}\left(t_{j}, \beta, \eta\right)$, the time-to-failure $\chi_{j}$ is generated as following:

$$
\chi_{j}=\eta\left(\left(\frac{t_{j}}{\eta}\right)^{\beta}-\ln (b)\right)^{1 / \beta}-t_{j}
$$

The generated times-to-failure are then compared with the time $k \tau$ of the soonest scheduled inspection flagged as ' 1 ' in $\boldsymbol{x}$. If $\chi_{j}<i \tau$, the number of failures of component $j$ is increased by 1. When the number of failed components accumulates to $n-k+1$ failures, the system fails, giving rise to opportunistic inspection, during which all of the failed components are discovered. A failed component is minimally repaired if its cumulative number of failures is $m$ or fewer; otherwise, it is replaced, and the cumulative number of its failures is reset to zero.

If $\chi_{j}<i \tau$, but the number of failed components is less than $n-k+1$, then the failed component is fixed at the next scheduled inspection. Again, if the component's cumulative number of failures is $m$ or fewer, it is minimally repaired; otherwise, the component is replaced, and the cumulative number of its failures is reset to zero.

If $\chi_{j}>i \tau$, the simulation clock is moved forward to the inspection time, since there is no failed component to be discovered at inspection.

The simulation clock is updated at the times of events, such as component failures, system failures, and scheduled and opportunistic inspections. The downtime of component $j$, the number of system failures, the number of minimal repairs and replacements are all updated at each event's time as well. At the same time, the ages of the surviving components, the time until the next failure, and the time until the scheduled inspection are also updated. The simulation stops when the end of the planning horizon is reached. The simulation model provides the expected random variables $F, M_{\boldsymbol{x}^{a}, m, j}, R_{\boldsymbol{x}^{a}, m, j}$ and $U_{\boldsymbol{x}^{a}, m, j}$ when run for a large number of times.

A given policy $\left(\boldsymbol{x}^{a}, m\right)$ prescribes the choice of the maintenance action at each simulation run. Varying the values of $\left(\boldsymbol{x}^{a}, m\right)$ in the ranges $\boldsymbol{x}^{a}=(0,0, \ldots, 0),(1,0, \ldots, 0), \ldots,(1,1, \ldots, 1), 0 \leq$ $m \leq U C L$, the total expected cost $E\left[C_{\boldsymbol{x} . m}^{T, k / n}\right]$ for policy $\left(\boldsymbol{x}^{a}, m\right)$ is computed and saved.

Finally, in order to find the optimal joint maintenance and inspection policy $\left(\boldsymbol{x}^{*}, m^{*}\right)$, the total expected costs are searched for the minimum total expected cost $E\left[C_{x^{*} . m^{*}}^{T, k}\right]$. From the 
optimal total expected $\operatorname{cost} E\left[C_{x^{*} . m^{*}}^{T, k, n}\right]$, the corresponding optimal non-periodic inspection policy $\boldsymbol{x}^{*}$ and the optimal number of minimal repairs before replacement $m^{*}$ are found, thus representing the joint maintenance and inspection policy $\left(\boldsymbol{x}^{*}, m^{*}\right)$ and satisfying the present objective.

\subsubsection{Simulation model for a system with hard-type and soft-type components (System 2)}

The general simulation procedure for System 2 is similar to that described for System 1. The following input variables are used: $x_{i}^{a}, m_{s}, \omega, \tau, t_{s}, \theta_{h}, \beta_{s}, \beta_{h}, \eta_{s}, \eta_{h}, \zeta_{h}, c_{s}^{M}, c_{h}^{D}, c_{s}^{R}, c_{h}^{D}, c_{s}^{D}$ and $c^{I}$. The same procedure as discussed in the previous section is used to generate the times for events and update the simulation clock.

If $\chi_{s}<\chi_{h}<i \tau$ for the generated soft failure time $\chi_{s}$, the closest hard failure time $\chi_{h}$ and the closest non-periodic inspection time $i \tau$, then an opportunistic inspection is occurring at the closest hard failure time. The soft failure is detected at this moment, and if the total number of previous failures is less than $m_{s}$, the component is minimally repaired; otherwise, it is replaced, and its cumulative number of failures is set to zero.

If $\chi_{h} \leq \chi_{s}<i \tau$, then the soft failure is fixed at the time of the soonest non-periodic scheduled inspection.

If $\chi_{h}<i \tau$, the age of the failed hard-type component is compared with its corresponding replacement age $\zeta_{h}$, and the hard-type component is replaced if $\theta_{h}>\zeta_{h}$, or it is minimally repaired otherwise. The component's age is set to zero at replacement.

Changing the values of a given joint inspection and maintenance policy $\left(\boldsymbol{x}^{a}, m_{s}, \zeta_{h}\right)$ in the ranges $\boldsymbol{x}^{a}=(0,0, \ldots, 0),(1,0, \ldots, 0), \ldots,(1,1, \ldots, 1), 0 \leq m_{s} \leq U C L_{s}, s=1,2, \ldots, n_{1}, 0 \leq \zeta_{h} \leq$ $1.5 \omega, h=1,2, \ldots, n_{2}$, the total expected cost for each policy is computed and saved.

Finally, the optimal solution is the one with the minimum cost $E\left[C_{\boldsymbol{x}^{*}, m_{S}^{*}, \zeta_{h}^{*}}^{T, H S}\right]$ over all input variables, i.e. inspection schemes, possible values of the number of minimal repairs before replacement for soft-type components and possible values for the optimal replacement ages for hard-type components. The resulting triple $\left(\boldsymbol{x}^{*}, m_{s}^{*}, \boldsymbol{\zeta}^{*}\right)$ represents the optimal joint maintenance and inspection policy. 
In using the simulation procedure described above, however, there is a significant drawback related to the search method used for optimisation. The exhaustive search method for finding the optimal joint policy is highly susceptible to the size of the problem, i.e. the choice of $\tau$, the upper confidence limit on the expected number of component failures, etc. Therefore, for systems with many components or larger planning horizons the search space and the simulation time increase dramatically. The problem's complexity also increases when considering a finer quasi-continuous space of possible inspection times with smaller $\tau$, as in this case the number of non-periodic inspections increases as well and the possible number of inspection schemes quickly explodes. Thus, based on all of these, there is a need for improving the efficiency and reducing the complexity of the computations.

In order to reduce the search space and the complexity of the problem, the number of total expected cost calculations should be decreased. The genetic algorithm provides a powerful heuristic search means to do this. This approach is further discussed below.

\subsection{Numerical Example}

The present section provides examples for each of the models developed in the preceding sections.

\subsubsection{Model 5: k-out-of-n system with opportunistic inspections}

We first consider a 3-out-of-5 redundant system with parameters given in Table 17. All components are assumed to be initially as-good-as-new.

Table 17: Parameters of the Power Law Intensity Function and Costs.

\begin{tabular}{llllllll}
\hline $\begin{array}{l}\text { Case } \\
\#\end{array}$ & $\beta$ & $\begin{array}{c}\eta \\
\text { (months) }\end{array}$ & $\begin{array}{l}\text { Minimal } \\
\text { repair } \\
\text { cost, } c^{M}\end{array}$ & $\begin{array}{l}\text { Replacement } \\
\text { cost, } c^{R}\end{array}$ & $\begin{array}{l}\text { Component } \\
\text { downtime } \\
\text { cost, } c^{D}\end{array}$ & $\begin{array}{l}\text { System } \\
\text { downtime } \\
\text { cost, } c^{S D}\end{array}$ & $\begin{array}{l}\text { Fixed } \\
\text { inspection } \\
\text { cost, } c^{I}\end{array}$ \\
\hline 1 & 1.5 & 3.5 & $\$ 75$ & $\$ 200$ & $\$ 60$ & $\$ 550$ & $\$ 50$ \\
\hline 2 & 1.5 & 3.5 & $\$ 75$ & $\$ 200$ & $\$ 80$ & $\$ 550$ & $\$ 50$ \\
\hline 3 & 1.5 & 3.5 & $\$ 75$ & $\$ 200$ & $\$ 60$ & $\$ 350$ & $\$ 50$ \\
\hline 4 & 1.5 & 3.5 & $\$ 75$ & $\$ 200$ & $\$ 60$ & $\$ 550$ & $\$ 100$ \\
\hline 5 & 1.5 & 5 & $\$ 75$ & $\$ 200$ & $\$ 60$ & $\$ 550$ & $\$ 50$ \\
\hline
\end{tabular}


Simulation and optimisation was performed using genetic algorithm for 5,000 runs with the planning horizon of 12 months and $\tau=1$ month. The output of the GA for Case 1 is given in Figure 16 below.
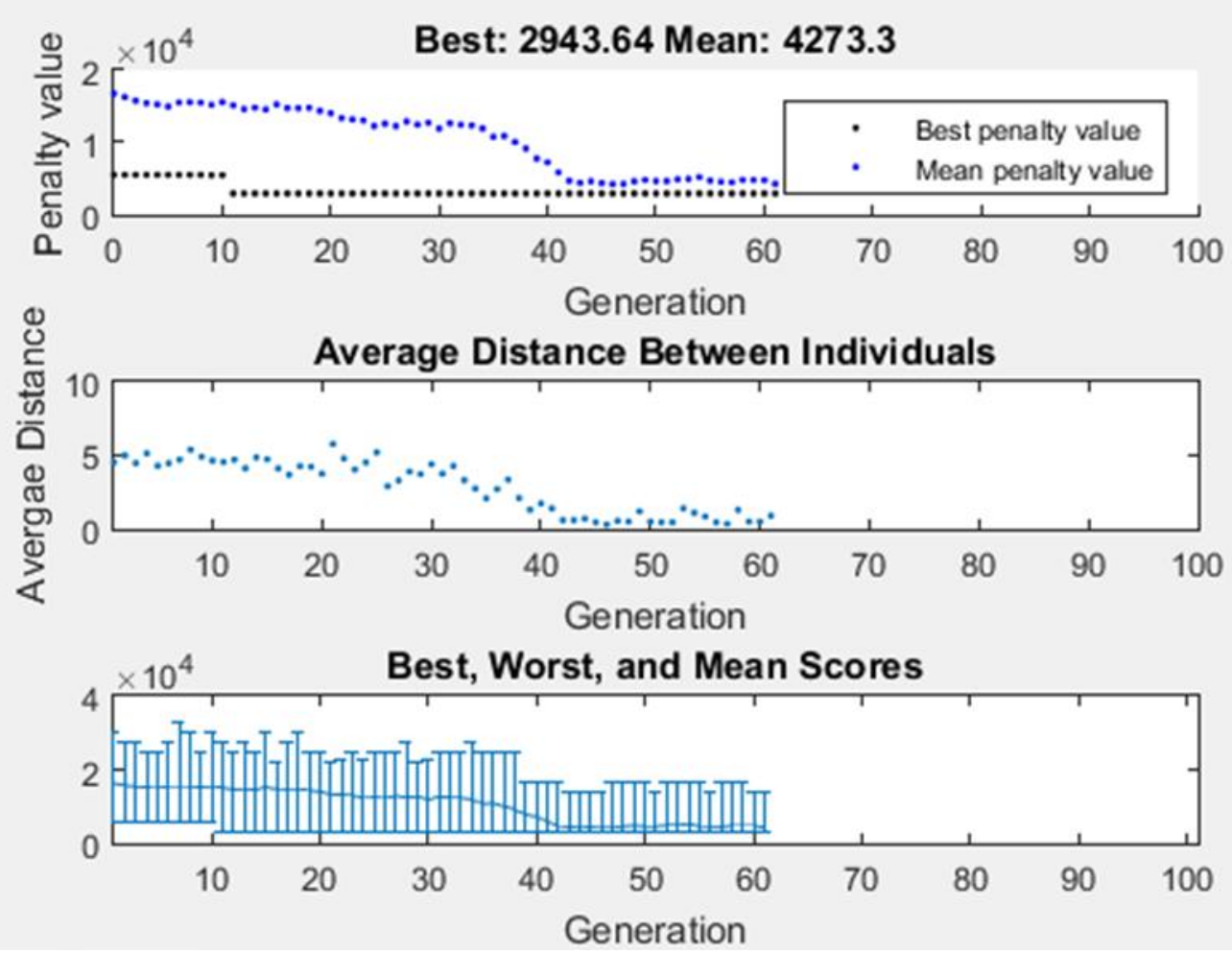

Figure 16: Genetic algorithm's output and results for Case 1 of a 3-out-of-5 system.

For the GA optimisation, the highest fitness value is equivalent to the lowest total expected cost. The genetic algorithm search used a limit of 100 generations, a stall generation limit of 50, an elite count of 1 and the tolerance limit of $10^{-5}$.

As can be seen from Figure 16, the best solution was found after 11 generations. The top graph in Figure 16 shows the output for the best and the mean total expected cost for each generation. As the number of generations increases, the GA-generated fitness function value gets closer to the optimal total expected cost and converges after about 40 generations.

The middle graph in Figure 16 displays the distances from each member of the population to its nearest neighbour. Generally, the smaller the distance, the faster the algorithm converges. 
The bottom graph in Figure 16 contains the best, worst and mean scores for the fitness function, i.e. the total expected cost. It can be seen that starting at generation 39 , the differences among these three measures become relatively small.

Baseline Case 1 was compared with the other cases for sensitivity analysis. The results of the genetic algorithm's optimisation procedure for the cases are given in Table 18 below.

Table 18: Optimal Policies from the Genetic Algorithm.

\begin{tabular}{|c|c|c|c|c|c|}
\hline $\begin{array}{l}\text { Case } \\
\#\end{array}$ & $\begin{array}{l}\text { Distinction } \\
\text { from baseline }\end{array}$ & $\begin{array}{l}\text { Total expected } \\
\text { cost, } E\left[C_{\boldsymbol{x}^{a} . m}^{T, k, n}\right]\end{array}$ & $\begin{array}{l}\text { Optimal inspection } \\
\text { policy, } \boldsymbol{x}^{*}\end{array}$ & $\begin{array}{l}\text { Optimal } \\
\text { maintenance } \\
\text { policy, } m^{*}\end{array}$ & $\begin{array}{l}\text { Number of } \\
\text { inspection } \\
\text { policies } \\
\text { analysed, } a\end{array}$ \\
\hline 1 & - & $\$ 2943.64$ & 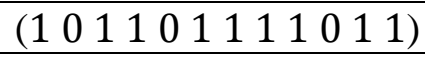 & 5 & 1755 \\
\hline 2 & $c^{D}=\$ 80$ & $\$ 3098.46$ & $\left(\begin{array}{llllllllllll}0 & 1 & 1 & 0 & 1 & 1 & 1 & 0 & 1 & 1 & 1 & 1\end{array}\right)$ & 6 & 1654 \\
\hline 3 & $c^{S D}=\$ 350$ & $\$ 2775.63$ & 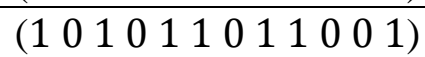 & 11 & 1709 \\
\hline 4 & $c^{I}=\$ 100$ & $\$ 3123.17$ & 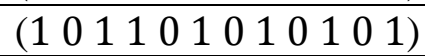 & 10 & 1638 \\
\hline 5 & $\eta=5$ & $\$ 1946.68$ & $\left(\begin{array}{llllllllllll}0 & 1 & 0 & 0 & 1 & 1 & 0 & 0 & 1 & 1 & 0 & 1\end{array}\right)$ & 7 & 1104 \\
\hline
\end{tabular}

As can be seen from Table 18, the optimal inspection policy suggests that for the baseline Case 1 system, 9 inspections should be performed at month 1, 3, 4, 6, 7, 8, 9, 11 and 12 within the 12-months planning horizon, and the optimal maintenance policy suggests that failed components should be replaced upon failure after having been minimally repaired for 5 times.

For the system with the component downtime cost of $\$ 80$ (Case 2), 9 inspections should be done at month $2,3,5,6,7,9,10,11$ and 12, and the optimal maintenance policy prescribes component replacement on $7^{\text {th }}$ failure within the 12-months planning horizon. Unsurprisingly, the total expected cost for Case 2 is higher than that for Case 1, since the cost of component downtime is higher for Case 2 than for Case 1.

For Case 3, the lower system downtime penalty results in the lower the total expected cost, fewer inspections and greater optimal number of minimal repairs before inspection as compared with Case 1. Lower system downtime penalty translates into more of allowable system downtime, which necessitates fewer inspections ( 7 for Case 3 vs. 9 for Case 1) and a much greater number of minimal repairs before replacement. The fact that the optimal number of minimal repairs before replacement is equal to the upper confidence limit on the expected number of failures implies that it is economically infeasible to replace failed components when the system downtime penalty is significantly decreased. However, the total effect of the 
decrease of the system downtime penalty by $\$ 200$ is reduced by the increased downtime as a result of the fewer inspections and greater component deterioration due to fewer replacements, all of which are reflected in the total expected cost's decrease of only $\$ 168.01$.

The optimal joint inspection and maintenance policies for Case 4 are close to those of Case 3, but the total expected cost is greater than that for either Case 3 or Case 1 . Unsurprisingly, increasing the cost of system inspection results in the increase in the total expected cost. Removing the effect of the total cost of inspection, it can be seen that the remaining expected cost for Case 4 is lower than that for Case 1 by $\$ 70.47$. This is likely the result of the 2-fold increase in the optimal number of minimal repairs before replacement, which results in fewer component replacements prescribed by Case 4 compared with those for Case 1.

Finally, for Case 5, the total expected cost is the lowest among all the tested cases. This can be explained by higher scale (spread) parameter of the time-to-failure distribution, which implies fewer failures within the same time interval for Case 5 compared to the other cases. Using Equation (11) from Babishin and Taghipour [87], the calculated expected number of system failures is approximately 5.7 for Cases $1-4$ and only about 2.6 for Case $5-$ a decrease by over $121 \%$ for Case 5 compared to the other cases. This also results in the fewest optimal number of inspections (6) among all the cases and, also, a slightly higher optimal number of

minimal repairs before replacement (7) and, correspondingly, fewer component replacements compared to that for Case 1.

\subsubsection{Model 6: system with hard-type and soft-type components and opportunistic inspections}

We consider a system composed of $m_{1}=5$ soft-type and $m_{2}=3$ hard-type components, all of which are initially "as-good-as-new". Two cases are considered: Case 1 (Baseline) and Case 2 (1.5-time greater monthly downtime penalty cost compared to Baseline). The input parameters for the failure distributions, the costs of minimal repair, replacement and downtime are given for both cases in Table 19. 
Table 19: Parameters of the power law intensity function and costs for different components of Case 1 (Baseline) and Case 2.

\begin{tabular}{|c|c|c|c|c|c|c|c|}
\hline \multicolumn{2}{|c|}{$\begin{array}{l}\text { Component } \\
\text { type }\end{array}$} & $\beta_{j}$ & $\begin{array}{c}\eta_{j} \\
\text { (months) }\end{array}$ & $\begin{array}{l}\text { Minimal } \\
\text { repair } \\
\text { cost, } c_{j}^{M}\end{array}$ & $\begin{array}{l}\text { Replacement } \\
\text { cost, } c_{j}^{R}\end{array}$ & $\begin{array}{c}\text { Case } 1 \\
\text { downtime } \\
\text { penalty } \\
\text { cost } / \text { month, } \\
c_{j}^{D}\end{array}$ & $\begin{array}{c}\text { Case } 2 \\
\text { downtime } \\
\text { penalty } \\
\text { cost } / \text { month, } \\
c_{j}^{D}\end{array}$ \\
\hline \multirow{5}{*}{ Soft } & 1 & 1.3 & 3.5 & $\$ 70$ & $\$ 200$ & $\$ 80$ & $\$ 120$ \\
\hline & 2 & 2.8 & 4.6 & $\$ 45$ & $\$ 150$ & $\$ 55$ & $\$ 82.5$ \\
\hline & 3 & 2.1 & 2.7 & $\$ 100$ & $\$ 300$ & $\$ 85$ & $\$ 127.5$ \\
\hline & 4 & 3.2 & 7.0 & $\$ 75$ & $\$ 240$ & $\$ 90$ & $\$ 135$ \\
\hline & 5 & 1.7 & 3.6 & $\$ 125$ & $\$ 325$ & $\$ 100$ & $\$ 150$ \\
\hline \multirow{3}{*}{ Hard } & 1 & 1.5 & 8.7 & - & - & - & - \\
\hline & 2 & 1.2 & 6.0 & - & - & - & - \\
\hline & 3 & 1.7 & 7.5 & - & - & - & - \\
\hline
\end{tabular}

The fixed cost of scheduled inspections $c_{I}=\$ 25$. Both cases are simulated for 1,000 runs with the planning horizon of 12 months and $\tau=1$ month. Lower number of simulation runs had to be used because of the much greater time required to run the simulation and the genetic algorithm for the hard-and-soft-type system compared to a $k$-out-of- $n$ system, which is a result of the former's greater complexity.

The output of the GA for Case 1 is given in Figure 17 below. The genetic algorithm search used a limit of 100 generations, a stall generation limit of 50, an elite count of 1 and the tolerance limit of $10^{-5}$.

As can be seen from the top graph in Figure 17, the best solution ("best penalty value") was found after 71 generations.

The middle graph in Figure 17 displays the distances from each member of the population to its nearest neighbour. Generally, the smaller the distance, the faster the algorithm converges.

The bottom graph in Figure 17 contains the best, worst and mean scores for the fitness function, i.e. the total expected cost. It can be seen that the mean scores of the fitness function are minimal at generations 92-94, owing to the lowest worst scores, while the best scores remain unchanged starting at generation 71 . 

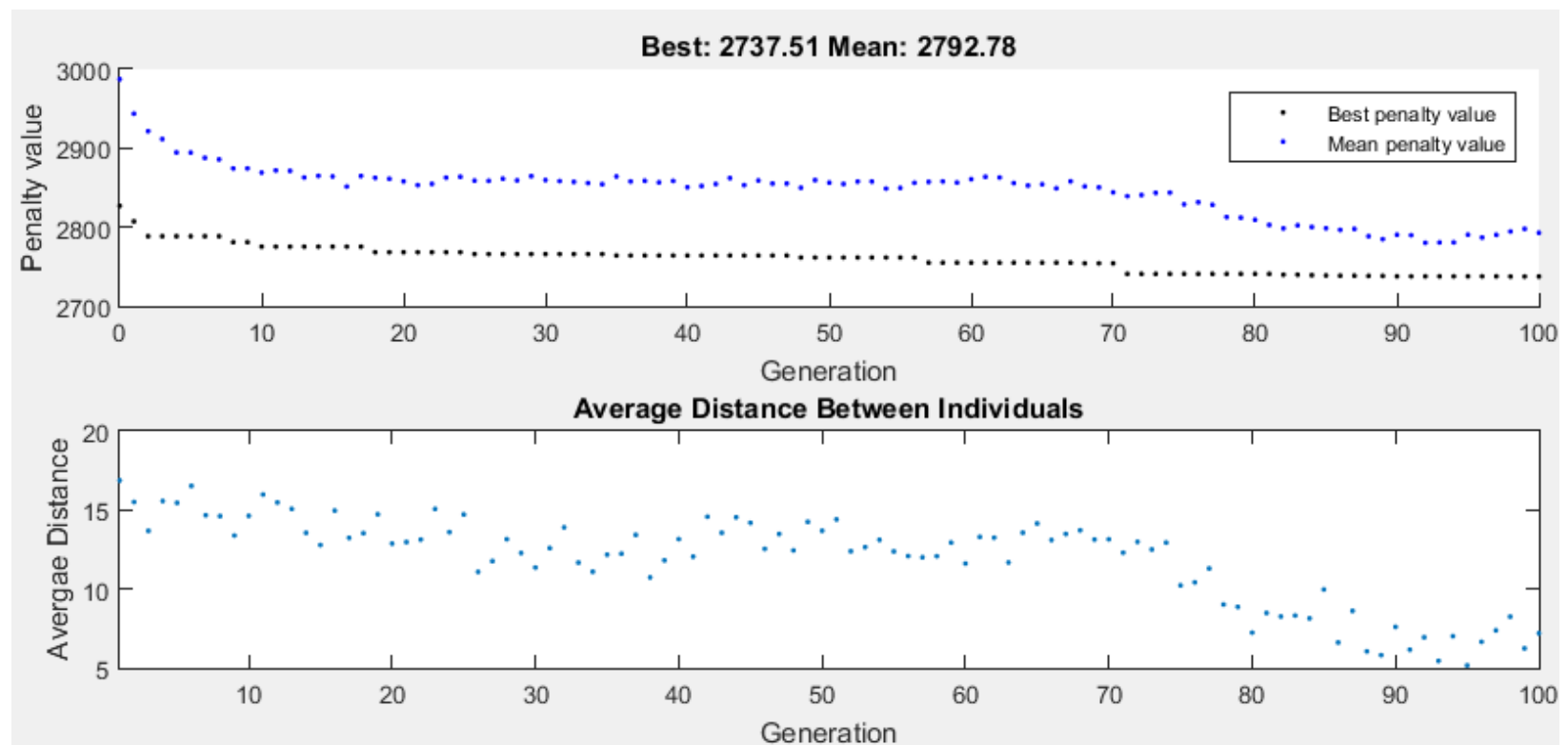

Best, Worst, and Mean Scores

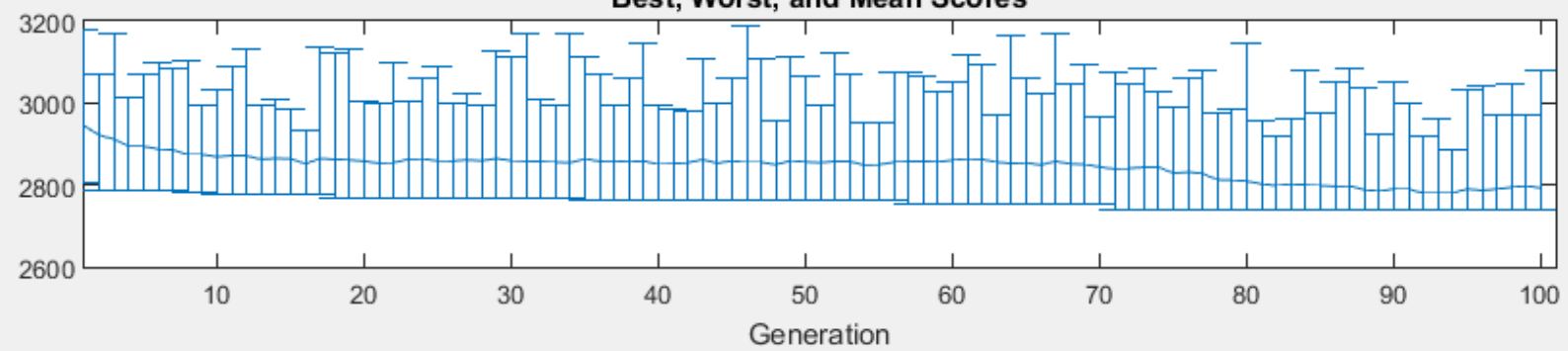

Figure 17: Genetic algorithm's output and results for Case 1 of a system composed of softand hard-type components.

The soft-type and the hard-type component parameters from Table 19 are used to optimise for the joint inspection and maintenance policies using genetic algorithm. The results are obtained for both Case 1 and Case 2. The optimal maintenance policies, i.e. the optimal numbers of minimal repair before replacement and the optimal replacement ages, are provided for both cases in Table 20 below. 
Table 20: Optimal maintenance policies for Case 1 and Case 2.

\begin{tabular}{|c|c|c|c|c|c|}
\hline $\begin{array}{c}\text { Case } \\
\#\end{array}$ & $\begin{array}{c}\text { Distinction from } \\
\text { baseline }\end{array}$ & $\begin{array}{c}\text { Soft-type } \\
\text { component, } \\
S\end{array}$ & $\begin{array}{l}\text { Optimal number of } \\
\text { minimal repairs before } \\
\text { replacement, } m_{s}^{*}\end{array}$ & $\begin{array}{c}\text { Hard-type } \\
\text { component, } \\
h\end{array}$ & $\begin{array}{c}\text { Optimal } \\
\text { replacement age, } \\
\zeta_{h} \text { (months) }\end{array}$ \\
\hline \multirow{5}{*}{1} & \multirow{5}{*}{ - } & 1 & 7 & 1 & 14.46 \\
\hline & & 2 & 1 & 2 & 8.25 \\
\hline & & 3 & 6 & 3 & 11.83 \\
\hline & & 4 & 8 & - & - \\
\hline & & 5 & 13 & - & - \\
\hline \multirow{5}{*}{2} & \multirow{5}{*}{$\begin{array}{c}c^{D}(\text { Case } 2)= \\
1.5 c^{D}(\text { Case } 1)\end{array}$} & 1 & 9 & 1 & 16.61 \\
\hline & & 2 & 1 & 2 & 6.77 \\
\hline & & 3 & 3 & 3 & 13.98 \\
\hline & & 4 & 0 & - & - \\
\hline & & 5 & 10 & - & - \\
\hline
\end{tabular}

As can be seen from Table 20, the optimal replacement ages for some of the hard-type components (namely, for hard-type component 1 for Case 1 and hard-type components 1 and 3 for Case 2) exceed the planning horizon. This simply means that these components would be replaced only at the end of the planning horizon and would be minimally repaired if they fail at any time until then. Also, changes in the optimal maintenance policies for both the soft-type and the hard-type components suggest that they are affected by changes in the component downtime penalty.

The resultant optimal inspection policy was also found for both cases to be as shown in Table 21 below.

Table 21: Optimal policies from the genetic algorithm.

\begin{tabular}{|c|c|c|c|}
\hline Case \# & $\begin{array}{l}\text { Total expected } \\
\text { cost, } E\left[C_{x^{a}, m_{s}, \zeta_{h}}^{T, H S}\right]\end{array}$ & $\begin{array}{l}\text { Optimal inspection } \\
\text { policy, } x^{*}\end{array}$ & $\begin{array}{l}\text { Number of inspection } \\
\text { policies analysed, } a\end{array}$ \\
\hline 1 & $\$ 2737.51$ & 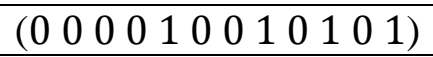 & 9935 \\
\hline 2 & $\$ 3848.41$ & $\left(\begin{array}{llllllllll}0 & 0 & 1 & 1 & 1 & 1 & 1 & 1 & 1 & 1\end{array}\right.$ & 9935 \\
\hline
\end{tabular}

The optimal inspection policy for Case 1 implies that the system is inspected 4 times in months 5, 8, 10 and 12. The optimal inspection policy for Case 2 is drastically different with 10 inspections occurring on a monthly basis in months 3-12. Thus, as a result of a 1.5-time increase in the per-component monthly downtime penalty cost, the optimal inspection policy alone for Case 2 costs $\$ 150$ more $(\$ 25 \cdot(10-4)$ inspections) than that for Case 1 . This leaves another 
$\$ 960.90$ as the increase in the cost of the optimal maintenance policy out of the total increase of $\$ 1110.90$ ( $\$ 3848.41-\$ 2737.51$ ) in the total expected cost for Case 2 compared to Case 1. Converting dollars into percentages, the total expected cost is over $40 \%$ greater for Case 2 compared for Case 1. A large reduction in the optimal number of minimal repairs before replacement for 3 out of 5 soft-type components is meant to decrease these components' downtime by reducing their ages to 0 with each replacement more frequently for Case 2 than for Case 1. The optimal number of minimal repairs before replacement, averaged across all softtype components, is 4.6 for Case 2 and 7 for Case 1 - a decrease of over $52 \%$ as a result of the downtime costs increasing by $50 \%$. At the same time, the optimal replacement ages of the hardtype components increased, on average, by only slightly over $8 \%$ (which is less than $\tau$ ) for Case 2 compared to Case 1 . These results are summarised in Table 22 below.

Table 22: Absolute and relative changes in the expected costs for Case 2 compared to Case 1.

\begin{tabular}{lllllll}
\hline $\begin{array}{l}\text { Change } \\
\text { (Case 2 }-\end{array}$ & $\begin{array}{l}\text { Average } \\
\text { system } \\
\text { Case 1) } \\
\text { downtime } \\
\text { penalty } \\
\text { cost }\end{array}$ & $\begin{array}{l}\text { Average } \\
\text { optimal } \\
\text { replacement }\end{array}$ & $\begin{array}{l}\text { Average optimal } \\
\text { number of } \\
\text { ainimal repairs } \\
\text { before } \\
\text { replacement }\end{array}$ & $\begin{array}{l}\text { Optimal } \\
\text { inspection } \\
\text { policy cost }\end{array}$ & $\begin{array}{l}\text { Optimal } \\
\text { maintenance } \\
\text { policy cost }\end{array}$ & $\begin{array}{l}\text { Total } \\
\text { expected } \\
\text { cost, } \\
E\left[C_{x^{a}, m_{s}, \zeta_{h}}^{T, H S}\right.\end{array}$ \\
\hline Absolute & $+\$ 205$ & +0.94 & -2.40 & $+\$ 150$ & $+\$ 960.90$ & $+\$ 1110.90$ \\
\hline Relative & $+50 \%$ & $+8.13 \%$ & $-52.17 \%$ & $+5.48 \%$ & $+35.10 \%$ & $+40.58 \%$ \\
\hline
\end{tabular}

As can be concluded from Table 22, the average optimal number of minimal repairs before replacement is the most sensitive to change in the downtime penalty, followed by the total expected cost and the optimal maintenance policy cost, with an increase in downtime penalty causing a decrease in the optimal number of minimal repairs before replacement and increase in each of the total expected cost and the optimal maintenance policy cost. On the contrary, the optimal inspection policy cost is the least sensitive, followed by the average optimal replacement ages, where an increase in downtime penalty increases each of the optimal inspection policy cost and the average optimal replacement ages.

Since failures are implicitly assumed to occur independently for different components within the same component type, it may be considered as a limitation if components are known to operate at full capacity. However, if the system is not strained (which is a realistic assumption for many $k$-out-of- $n$ systems), this may not necessarily be a limitation, since components are able to "absorb" the increased operational load due to failure(s). 


\subsection{Conclusion}

In the present chapter, the problem of non-periodic inspection and maintenance optimisation was considered for complex multicomponent systems with either $k$-out-of- $n$ redundant configuration, or with components prone to hard and soft failures. Aside from scheduled inspections, components can be checked opportunistically at system failures. Making the unit of time sufficiently small allows to treat the planning horizon as quasi-continuous for possible non-periodic inspections, which gives a much greater flexibility and variety in the choice of available inspection policies at the expense of computational complexity when compared to the periodic inspections. Since soft failures are hidden, component's age cannot be used as the criterion for maintenance optimisation. Instead, maintenance policies are defined by the number of minimal repairs before replacement for each component prone to hidden soft failure. The optimal policies are then found by jointly optimising the inspection and maintenance policies for the lowest total expected cost. Using simulation and a genetic algorithm to implement the joint optimisation was found to be an efficient and convenient method to find the optimal policies for large and complex systems. This appears to be a promising method for multiobjective optimisation with regard to complex systems.

\section{Acknowledgements}

The author acknowledges the financial support from the Natural Sciences and Engineering Research Council (NSERC) of Canada for this research. 


\section{Conclusions and Future Research}

\subsection{Main Results and Contributions}

Devising effective and efficient inspection and maintenance policies for real-world systems, most of which are complex, is often a daunting task. Methods, techniques and approaches both theoretical and practical - that have been proposed in the past, usually consider only simple systems consisting of very few components, or impose oversimplifying assumptions on the systems of interest, thus decreasing the practical value of such developments and hindering their real-world application and implementation. The need for models that are able to capture the richness of various scenarios that can occur in practice is especially important for the fields where system failures are associated with a great risk and large costs, such as in healthcare and medical industries. Ensuring the reliable operation of devices and systems designed to support, treat or rehabilitate patients, such as, for example, infusions pumps, is an important problem. Improvements in this area are likely to result not only in the reduction of the risk to the health and well-being of patients, but also in significant, if not dramatic, cost savings to hospitals, care providers, governments and the general public.

Because of the risks and high potential costs of failure, devices used in the healthcare industry need to be reliable, effective and available. Most of these devices are usually complex and consist of many different components, which exhibit interactions among the components, as well as interactions with external environment. For this reason, it is usually hard to predict when failures of such systems will occur and, consequently, how to best manage remediation of the latter. This, in turn, puts weight on inspection and maintenance in providing means to operate these systems at the lowest possible risk and costs. It is very difficult, if not impossible to foresee or predict all of the risks pertaining to the above-mentioned settings and situations, let alone quantifying these risks. For this reason, one of the most popular metrics used for optimisation is the cost of inspection and maintenance.

The goal of the research presented in this dissertation is to propose models that improve and facilitate inspection and maintenance methods used for multicomponent systems. The proposed models are meant to incorporate fewer restrictive and oversimplifying assumptions and, thus, to be closer in description to an actual wide range of systems encountered in practice. In all of 
the models, components are assumed to follow a non-homogenous Poisson process with a power law intensity function. A finite system life cycle (planning horizon) is considered for all models.

For the periodic inspection approach, two models are developed for the systems composed of hard-type and soft-type components, and two models for the $k$-out-of- $n$ systems. The models vary depending on the assumptions made. The main contributions of Models 1 to 4 include: (1) joint optimisation of periodic inspection frequency and maintenance action for a multicomponent system and the development of mathematical models that are solved to find the expected values in the objective functions, (2) reduction of the minimal intervals at which a scheduled inspection can be performed - this leads to a semi-continuous variable for the inspection interval, and (3) the use of the genetic algorithm to improve the computational efficiency, so that the optimal solution can be obtained in a shorter time.

The major contributions of the present dissertation can be classified as following.

(a) Model 1: this model considers a $k$-out-of- $n$ system subject to hidden component failures, which are revealed either at inspections, or system failures. We take into account both the periodic and opportunistic inspections occurring at the times of system failure over a finite life cycle. Since failures of components are discoverable only at either scheduled, or opportunistic inspections and are, therefore, hidden, the components' age cannot serve as the maintenance optimisation criterion. Instead, the number of minimal repairs before replacement is used for this purpose. Inspection policy is optimised on the basis of the length of the inspection period (i.e. time between two consecutive periodic inspections).

The objective function is formulated in terms of the total expected cost of system's inspection and maintenance. We jointly optimise for inspection and maintenance policies. Optimisation is performed using a Monte Carlo simulation with exhaustive search of the joint inspection and maintenance policy space. Sensitivity analysis shows that the objective function and, therefore, the joint inspection and maintenance policy are most sensitive to the inspection period length.

We also compare the model with the cases of series and parallel systems. The results suggest that due to the highest total system downtime penalty incurred, series systems have the highest total expected cost and result in an optimal joint policy with the longest inspection period and lowest number of minimal repairs before replacement (essentially, replace-upon-failure policy). 
The optimal number of minimal repairs before replacement is the lowest for parallel systems, since the number of failures, minimal repairs and replacements is generally lower for these systems compared to $k$-out-of- $n$ systems.

This model is applicable to redundant brake lines in cars, where the presence of multiple brake line contours ensures that as long as one line remains operational, there is still a pair of diagonally-opposite front and rear wheels with operational brakes. This model can also be applied to uninterruptible power supplies with active redundancy.

(b) Model 2: this model considers a redundant $k$-out-of- $n$ system subject to hidden component failures with preventive replacement of components at periodic inspections, as well as corrective maintenance (minimal repair or replacement) of components at periodic inspections and system failures.

By using the number of minimal repairs before replacement as the maintenance decision criterion, the model from Chapter 3 allows for preventive replacements of components even if their failures are hidden.

Optimisation is performed to find the optimal joint inspection and maintenance policies first by using the Monte Carlo simulation, which finds the optimal joint policy by exhaustively searching the policy space. We then use a genetic algorithm implementation, with an integer inspection period length, which provides identical solutions to the exhaustive search technique, but in a much shorter time.

We then demonstrate the method's applicability to cases where the minimum time between inspections may be smaller and/or the planning horizon may be much larger relative to the minimal inspection period length. Rather than rescaling the problem to represent the minimal inspection period as an integer and the planning horizon as its integer multiple, the increased efficiency of the genetic algorithm allows to consider very small inspection periods, which essentially makes the set of available periodic inspection times quasi-continuous, i.e. relaxing the integer constraint on the length of inspection period. Thus, the value of the length of the inspection period is no longer limited to the integer number of time units (e.g. months), but may also take positive rational values. 
The results of the sensitivity analysis suggest that the optimality of the joint inspection and maintenance policy is most sensitive to variation in the system's downtime penalty cost, since this is usually the cost with the largest absolute value. We plot and determine the empirical linear relation between the system downtime penalty cost and the total expected cost in the objective function for the optimal joint inspection and maintenance policy. This allows the development of a deterministic linear empirical model for the total expected cost in terms of the system downtime penalty cost, thus demonstrating the method of constructing a simple deterministic model restricted to a set of parameters from a general stochastic model.

The value of the objective function is found to be negatively sensitive to changes in the length of the optimal inspection period. We also find that sensitivity of the objective function to the optimal inspection period increases as the length of the inspection period decreases. As the optimal inspection period length decreases, its sensitivity to the system downtime penalty also decreases. This is because longer inspection period, on average, causes the system to remain in the failed state for a longer time. This, in turn, incurs greater costs due to the higher system downtime. Thus, system downtime penalty favours smaller periodic inspection interval in order to retain the optimality of the total expected cost and, consequently, of the objective function.

The model described in this section can be applied, for example, to arrays of general purpose relays as encountered in factory settings.

(c) Model 3: this model features corrective maintenance (minimal repair or replacement) of hard-type and soft-type components, and opportunistic and periodic inspections of soft-type components. Both hard-type and soft-type components are subject to opportunistic inspections in addition to the scheduled periodic inspections over a finite life cycle.

Although soft failures are hidden and revealed only at periodic or opportunistic inspections, they degrade the system's performance. Hidden failures do not allow for the formulation of a closed-form mathematical expression.

The objective is to minimise the total expected cost of inspection and maintenance for the entire system. Because of the increased complexity of the problem, optimisation is performed using the three-stage Monte Carlo simulation. The first stage finds the optimal maintenance policy for the hard-type components, based on their replacement ages. The second stage finds the optimal maintenance policy for the soft-type components, based on the number of minimal 
repairs before replacement. The third stage finds the optimal inspection policy based on the optimal maintenance policy for the soft-type components. Recursive expressions for generating the expected values in the simulation are provided in the present thesis.

This models is used to optimise for the inspection and maintenance of a general infusion pump found in hospitals. In such pumps, fuses and displays are represented as hard-type components, and liquid-level alarm and battery are represented as soft-type components.

(d) Model 4: in addition to the assumptions listed for Model 3, the possibility of preventive replacement of hard-type components at periodic inspections is considered. In both Models 3 and 4, the occurrence of hard failures may impact the expected number of soft failures, and thus, the expected number of minimal repairs, replacements and expected downtime. Consequently, these expected values influence the length of the optimal periodic inspection interval. In Model 4, the costs of hard-type components can impact the optimal periodic inspection interval as well.

For the non-periodic inspection approach, we develop one model for a $k$-out-of- $n$ system and one model for a system composed of hard-type and soft-type components. The main contributions of these models include: (4) joint optimisation of non-periodic inspection and maintenance (minimal repair or replacement) policies for the systems, (5) formula for the expected number of system failures in terms of the cost ratio and component failure intensity, (6) simple formula for reliability of $k$-out-of- $n$ system, (7) formula for finding the planning horizon length based on the expected number of system failures, (8) proof outline of convexity of the total expected cost formula in the most general case.

The non-periodic models and other contributions are considered in more detail below.

(e) Model 5: in this model, we consider a redundant $k$-out-of- $n$ system subject to scheduled non-periodic and opportunistic inspections, the latter occurring at system failures. The available maintenance actions are either a minimal repair, or a corrective replacement. Inspection policies are based on the unique inspection schemes generated by representing decimal numbers as binary numbers having the length dependent on the planning horizon. Maintenance policies are based on the number of minimal repairs before replacement. Joint inspection and maintenance optimisation is performed using the genetic algorithm. 
An example of application of this model to the real-world situation is in optimising inspection and maintenance of several diesel generators providing electrical power to a remote base station (e.g. used in geophysical surveys), which because of crew staffing shortages may require non-periodic inspection and maintenance optimisation.

(f) Model 6: this model features corrective maintenance (minimal repair or replacement) of hard-type and soft-type components, and inspections of soft-type components at scheduled and opportunistic inspections. The soft-and-hard-type system considered here is subject to scheduled non-periodic and opportunistic inspections, the latter occurring at system failures. We consider corrective maintenance (minimal repair or replacement) of the components at scheduled inspections and at system's failures (opportunistic inspections). Maintenance policies for the hard-type components are based on the replacement age, generated from the interval bounded by 0 and some rational positive multiple of the planning horizon length. Maintenance policies for the soft-type components are based on the number of minimal repairs before replacement.

An application of this model includes a MacPherson-type strut assembly found in cars, where a shock absorber, a coil spring and a strut-to-mount nut are modelled as hard-type components, and lower and upper spring insulators, dust shield, jounce bumper and spring seat pad are modelled as soft-type components.

(g) For $k$-out-of- $n$ systems, we provide expression for the expected number of system failures in terms of the cost ratio. We also provide expressions for the expected number of system failures in terms of the component failure intensity and for calculating system reliability whenever considering the system's evolution until its first repair. In addition, we derive a criterion for establishing an acceptable level of expected system failures over the system's life cycle. The latter may be useful for planning the system's operating horizon, at the system design stage and when analysing its performance.

(h) We also demonstrate convexity of the objective functions used for optimisation in the proposed models. We provide a mathematical argument, as well as present three-dimensional plots for the objective functions in the case of periodic $k$-out-of- $n$ systems. 


\subsection{Future Work}

The models proposed in the present dissertation can be extended by incorporating more realistic or relaxing restrictive assumptions, taking into account additional considerations, employing new methods and techniques, analysing the problem in the context of new theories, etc. Some of the proposed directions for future work are given below.

(a) The most logical extension of the current work is to incorporate preventive replacements in the proposed non-periodic models. Conditions similar to those found in the periodic models can be used. Thus, for $k$-out-of- $n$ systems, components may be preventively replaced at scheduled inspections when their number of minimal repairs before replacement is equal to the optimal number of minimal repairs before replacement. For systems made up of hard- and softtype components, preventive replacements of hard-type components can be performed at scheduled non-periodic inspections whenever the component's age exceeds its optimal replacement age.

(b) Environmental risk factors (e.g. temperature, vibration, friction, pressure, humidity, etc.), which are important for such systems as turbines, engines, or chemical reactors, can be incorporated into the models by modifying the failure intensity function. The power law intensity function can be augmented by an exponential factor, whose power is a weighted sum of the risk factor covariates, thus making it similar to the form of the failure intensity function used in proportional hazards models.

(c) Combining the joint optimisation of inspection, maintenance and inventory may also be considered in a future work (see, for example, Salameh and Jaber [127], Bjarnason and Taghipour [21]). Of special interest are cases including both integer and quasi-continuous inspection periods. This problem may be especially suitable for genetic algorithm implementation because of its large search space.

(d) In the present treatment of multicomponent systems, the duration of inspections was considered negligible relative to the length of the planning horizon, and the technicalities of inspection are left out of scope. However, exactly what constitutes an inspection and how the inspection and maintenance processes take place are some of the most important practical aspects. Considering who and how inspects and maintains the system leads to a different dimension of multicomponent systems analysis. If inspection and/or maintenance are performed 
by different people, human, environmental and many other factors will contribute to the variability of these processes. In industrial operations, where workers often perform inspection and maintenance, the experience of individual workers may play a crucial role in the ultimate quality, reliability and maintainability of the operations or product. Wherever experience is important, training and the ability of staff to learn and re-learn their functions, responsibilities and procedures becomes fundamental in optimising inspection and maintenance. The processes of memory training, learning and forgetting emerge as very important in this regard (see, for example, Jaber [128]). Joining the methods from the literature on learning and forgetting processes with the work in inspection and maintenance optimisation is a promising area of future research.

(e) Inspecting and maintaining complex systems in high-stress environments with frequent lack of sufficient staffing characteristic of many hospital settings, has not been considered in the literature as widely as the design and manufacturing phases of system development. While the latter are, undoubtedly, very important, there is usually a wide gap between the designed and the actual operational conditions and environment. This causes excessive stress on the devices and systems and results in greater number and increased frequency of failures - both at the system and component levels.

Thus, complex systems and devices may be improved by observing their operation and use, including inspection and maintenance, in reality, and then using this information as a feedback for the (re-)design and improvement of these systems at the initial design stages. Human factors, in particular ergonomics, engineering psychology and human performance are of particular interest, since human error often leads to catastrophic consequences. Usability evaluations have been performed on infusion pumps (Schnittker et al. [129]), defibrillators (Fidler and Johnson [130], Fairbanks et al. [131], Fairbanks and Caplan [132]), etc. Incorporating the results of the usability analyses with the optimisation models for inspection and maintenance would attempt approaching the problem from both ends - the initial design stage, and the final usage stage. This may result in significant improvements on the designs of such devices and systems, while also reduce the costs associated with their operation.

(f) Another direction for the future work may be taken towards analysing the systems where the underlying hazard function is not deterministic, but instead is given by a random variable. 
Such processes fall under the generalisation of doubly-stochastic (Cox) processes and have found some applications in biophysics and physical chemistry [133], neuroscience [134] and astrophysics [135]. For example, photon arrival data in single-molecule biophysical experiments related to protein dynamics has been studied by Zhang and Kou [136]. Perhaps, application of the Cox process may be useful for studying the birth-and-death and/or renewal processes in maintenance and inspection optimisation of multicomponent/multiagent systems applicable to a wider range of physical phenomena.

(g) Yet another direction of possible work is in considering the problem from the point of view of the queueing theory, in particular hidden Markov models/chains and Markov decision processes (Neuman and Bonhomme [137], Xiang et al. [138]). More complex systems with hidden soft-type components having several states and associated transition probabilities may be considered. Using this framework will likely results in more emphasis being put on the degradation process of these components. For this reason, such analysis might be more useful or suitable to systems where degradation of components is of particular significance. Examples of such systems may include jet engines (Sun et al. [91]), turbines, generators (Jia [95], Tu et al. [92]), compressors (Zhou et al. [94]), motors, etc. Another class of systems might be the ones where their state is stochastically dependent on the observed state (e.g. obtained measurement from a sensor). This applies primarily to complex or expensive systems with electronic components relying on electromagnetic measurements, such as magnetometers and various geophysical surveying equipment. 


\section{Appendices}

\section{Appendix A. Convexity of the objective function}

The convexity of the objective function, i.e. the total expected cost function, can be ascertained by the following reasoning. Looking at the expressions for the total expected cost functions for either Type 1, or Type 2 systems, it can be seen that they are a summation composed of the deterministic and stochastic parts. The deterministic part is the sum of the costs of inspection and system downtime, and the stochastic part is the sum of the expected values of the costs of minimal repair, replacement and component downtime. It is known that the sum of the convex functions is also a convex function. In this case, the total expected cost function is convex if the deterministic and the stochastic parts of it are convex. We then consider each of the parts separately.

In the most general case, the deterministic part $\left(l c^{I}+F c^{S D}\right)$ is a sum of the linear functions. Since linear functions are simultaneously convex and concave, it follows that their sum and, consequently, the deterministic part, is convex.

In the most general case, the stochastic part is a sum of the products of constant costs and expected values. It can be noted that the expected number of failures (both for a component, and for the whole system) generally equals the sum of the expected numbers of minimal repairs and corrective replacements. Since the failures follow non-homogeneous Poisson process, the number of failures is Poisson-distributed with mean in the form of a power-law function of time (see Section 3.3 for derivation). Differentiating the expected value function twice, we obtain that its second derivative is non-negative, which is equivalent to the function's being convex. Since the number of failures is Poisson-distributed, the numbers of minimal repairs and corrective replacements are each Poisson-distributed (following the infinite divisibility property of the Poisson distribution). Hence, these random variables have the same form of the expected function and the preceding development regarding their convexity applies.

The most general form of the objective function used in the present work is given as following: 


$$
\begin{gathered}
E\left[C^{T}\right]=l c^{I}+F c^{S D}+\sum_{s=1}^{m_{1}}\left(c_{s}^{M} M_{s}+c_{s}^{R}\left(R_{s}+P R_{s}\right)+c_{S}^{D}\left(\omega-U_{s}\right)\right) \\
+c_{O I} \sum_{h=1}^{m_{2}}\left(M_{h}^{H}+R_{h}^{H}\right)+\sum_{h=1}^{m_{2}}\left(c_{h}^{M} M_{h}^{H}+c_{h}^{R}\left(R_{h}^{H}+P R_{h}^{H}\right)\right) .
\end{gathered}
$$

Considering the expected number of minimal repairs and the expected number of corrective replacements for each hard-type component:

$$
M_{h}^{H}+R_{h}^{H}=E\left[f_{h}\right]
$$

Similarly, for each soft-type component:

$$
M_{s}+R_{s}=E\left[f_{s}\right]
$$

Since each of the soft and hard failures follow NHPP by assumption, the number of each of the soft and hard failures is Poisson-distributed, and the time between failures is Weibulldistributed. The numbers of soft and hard failures can be regarded as splitting of the Poisson process describing the arrival of component failures.

For the number of system failures (since they occur at hard failure times), the following holds:

$$
\sum_{h=1}^{m_{2}}\left(M_{h}^{H}+R_{h}^{H}\right)=\sum_{h=1}^{m_{2}} E\left[f_{h}\right]=F
$$

Since the number of hard failures can be regarded as a splitting process of the number of system failures, it follows that the latter is also Poisson-distributed. Using the form of the expected number of a Poisson random variable, we check the deterministic part of objective function for convexity:

$$
\frac{\partial^{2}}{\partial t^{2}}\left(l c^{I}+F c^{S D}\right)=0+\frac{d^{2}}{d t^{2}}\left(\left(\frac{t}{\eta}\right)^{\beta} c^{S D}\right)=c^{S D} \frac{d}{d t}\left(\frac{\beta}{\eta}\left(\frac{t}{\eta}\right)^{\beta-1}\right)=c^{S D} \frac{\beta(\beta-1)}{\eta^{\beta}} t^{\beta-2} .
$$

But $c^{S D} \geq 0$, so dividing the preceding by $c^{S D}$ and setting it to be non-negative, obtain:

$$
\frac{\beta(\beta-1)}{\eta^{\beta}} t^{\beta-2} \geq 0 \Leftrightarrow\left\{\begin{array}{l}
t \geq 0 \\
\beta \neq 0,1 \\
\eta \neq 0
\end{array}\right.
$$


But all of these conditions follow from the properties of a Weibull distribution (for $\beta=1$, Weibull distribution turns into memoryless exponential distribution) and are, thus, satisfied. Therefore, it follows that the deterministic part of the objective function is convex.

To show that the stochastic part is convex, it is only necessary to show that each of its linear terms is convex. Since all of the multipliers-costs are non-negative and constant, they do not affect convexity of the objective function. We can then focus on showing convexity of the expected numbers in the stochastic part of the objective function. Equations (A.2) and (A.3) suggest that the number of minimal repairs and the number of corrective replacements all form splitting of a Poisson process, which implies they are also Poisson-distributed. In this case, reasoning from Equations (A.5) and (A.6) applies, and it follows that the expressions for these expected numbers are also convex.

The only term left that requires showing convexity is the downtime $\left(\omega-U_{s}\right)$. But, since $=$ const. and $\left(\omega-U_{s}\right) \geq 0$, showing convexity for downtime is equivalent to showing that for uptime $U_{s}$. Following from the assumption of NHPP and its properties, $U_{s}$ is Weibulldistributed, and the expected uptime can be given by the following:

$$
U_{s}=\eta \Gamma\left(1+\frac{1}{\beta}\right)
$$

Checking for convexity:

$$
\frac{\partial^{2}}{\partial t^{2}} U_{s}=\frac{d^{2}}{d t^{2}}\left(\eta \Gamma\left(1+\frac{1}{\beta}\right)\right)=0
$$

Therefore, it follows that the expected uptime is strictly convex. 


\section{Appendix B. Recursive formulations for the case of corrective maintenance of hard- type components and opportunistic inspections of soft-type components}

Following the same notations and steps as Taghipour and Banjevic [8], we consider four different scenarios which may take place in an inspection interval:

- A soft failure occurs before a hard failure

- A hard failure occurs and no soft failure happens before it

- A soft failure happens and no hard failure occurs after it before the periodic inspection time

- No soft and hard failures happen in the inspection interval

We consider soft-type component $s$ and a hard subsystem consisting of all hard-type components in series configuration with the hazard rate of $\lambda^{H}(z \mid \boldsymbol{\theta})=\sum_{h=1}^{n_{2}} \lambda_{h}\left(\theta_{h}+z\right)$. Let us assume $\omega=\tau$, which implies we have only one periodic inspection taking place at time $\tau$. We define $G_{1, s}\left(\tau, t_{s}, \boldsymbol{\theta}, \zeta^{*}, m_{c}, m_{s}\right)$ to be the function which provides us the expected value of a random variable of interest, such as the number of minimal repairs, replacements, or the downtime of soft-type component $s$ with initial age $t_{s}$, assuming at the beginning of the interval, the current number of minimal repairs already performed is $m_{c}$, and $m_{s}$ is the maximum number of minimal repairs before replacement for the component. $\boldsymbol{\theta}$ is the vector of initial ages of all hard-type components. In order to provide a universal form for the function for calculating different expected values, we define function $\psi\left(y, z, I_{s}\right)$ to act as a placeholder for the expected value of interest. $I_{S}$ is 0,1 , or 2 , if component $s$ is minimally repaired, replaced, or it is not failed in an interval, respectively. Assuming the first failure times of soft-type component $s$ and the hard subsystem are $Y$ and $Z$, correspondingly, and their density and reliability functions are $f^{Y}\left(y \mid t_{s}\right), f^{Z}(y \mid \boldsymbol{\theta}), \mathcal{R}^{Y}\left(y \mid t_{s}\right)$ and $\mathcal{R}^{Z}(z \mid \boldsymbol{\theta})$; we now formulate the expected value of the random variable of interest over inspection interval $[0, \tau]$ using the conditional expected value given $Y=y$ and $Z=z$ :

$G_{1, s}\left(\tau, t_{s}, \boldsymbol{\theta}, \zeta^{*}, m_{c}, m_{s}\right)$

$=\int_{0}^{\tau} \int_{0}^{z} \sum_{h=1}^{n_{2}}\left\{\left(\left[\psi(y, z, 0)+G_{1, s}\left(\tau-z, t_{s}+y, \boldsymbol{\theta} \oplus z, \zeta^{*}, m_{c}+1, m_{s}\right)\right] r_{s}^{Y}\left(m_{c}, m_{s}\right)\right.\right.$

$\left.+\left[\psi(y, z, 1)+G_{1, s}\left(\tau-z, 0, \boldsymbol{\theta} \oplus z, \boldsymbol{\zeta}^{*}, 0, m_{s}\right)\right] \bar{r}_{s}^{Y}\left(m_{c}, m_{s}\right)\right) r_{h}^{Z}\left(\theta_{h}+z, \zeta_{h}^{*}\right)$ 


$$
\begin{aligned}
& +\left(\left[\psi(y, z, 0)+G_{1, s}\left(\tau-z, t_{s}+y,(\boldsymbol{\theta} \oplus z)^{\left(0_{h}\right)}, \zeta^{*}, m_{c}+1, m_{s}\right)\right] r_{s}^{Y}\left(m_{c}, m_{s}\right)\right. \\
& \left.+\left[\psi(y, z, 1)+G_{1, s}\left(\tau-z, 0,(\boldsymbol{\theta} \oplus z)^{\left(0_{h}\right)}, \zeta^{*}, 0, m_{s}\right)\right] \bar{r}_{s}^{Y}\left(m_{c}, m_{s}\right)\right) \bar{r}_{h}^{Z}\left(\theta_{h}\right. \\
& \left.\left.+z, \zeta_{h}^{*}\right)\right\} q^{h}(z) f^{Y}\left(y \mid t_{s}\right) f^{Z}(z \mid \boldsymbol{\theta}) d y d z \\
& +\int_{0}^{\tau} \sum_{s=1}^{n_{1}}\left\{\left[\psi(z, z, 2)+G_{1, s}\left(\tau-z, t_{s}+z, \boldsymbol{\theta} \oplus z, \zeta^{*}, m_{c}, m_{s}\right)\right] r_{h}^{Z}\left(\theta_{h}+z, \zeta_{h}^{*}\right)\right. \\
& +\left[\psi(z, z, 2)+G_{1, s}\left(\tau-z, t_{s}+z,(\boldsymbol{\theta} \oplus z)^{\left(0_{h}\right)}, \zeta^{*}, m_{c}, m_{s}\right)\right] \bar{r}_{h}^{Z}\left(\theta_{h}\right. \\
& \left.\left.+z, \zeta_{h}^{*}\right)\right\} q^{h}(z) \mathcal{R}^{Y}\left(z \mid t_{s}\right) f^{Z}(z \mid \boldsymbol{\theta}) d z \\
& +\int_{0}^{\tau}\left[\psi(y, \tau, 0) r_{s}^{Y}\left(m_{c}, m_{s}\right)+\psi(y, \tau, 1) \bar{r}_{j}^{Y}\left(m_{c}, m_{s}\right)\right] f^{X}\left(x \mid t_{j}\right) d y R^{Z}(\tau \mid \boldsymbol{\theta}) \\
& +\psi(\tau, \tau, 2) \mathcal{R}^{X}\left(\tau \mid t_{j}\right) \mathcal{R}^{Z}(\tau \mid \boldsymbol{\theta}),
\end{aligned}
$$

where

$$
r_{s}^{Y}\left(m_{c}, m_{s}\right)=\left\{\begin{array}{l}
1, \text { if } m_{c} \leq m_{s} \\
0, \text { otherwise }
\end{array}, \quad r_{h}^{Z}\left(z, \zeta_{h}^{*}\right)=\left\{\begin{array}{c}
1, \text { if } z \leq \zeta_{h}^{*} \\
0, \text { otherwise }
\end{array}, \bar{r}_{s}^{Y}=1-r_{s}^{Y}, \quad \bar{r}_{h}^{Z}=1-r_{h}^{Z}\right.\right.
$$

The placeholder function $\psi\left(y, z, I_{s}\right)$ varies depending on the random variable of interest.

For the expected number of minimal repairs:

$$
\psi\left(y, z, I_{s}\right)=\left\{\begin{array}{l}
1, \text { if } I_{s}=0 \\
0, \text { otherwise }
\end{array}\right.
$$

For the expected number of replacements:

$$
\psi\left(y, z, I_{s}\right)= \begin{cases}1, & \text { if } I_{s}=1 \\ 0, & \text { otherwise }\end{cases}
$$

For the expected uptime:

$$
\psi\left(y, z, I_{s}\right)= \begin{cases}y, & \text { if } y<z \\ z, & \text { otherwise }\end{cases}
$$


$q^{h}(z)$ is the probability that the failure of the hard subsystem at time $Z=z$ is due to the failure of hard-type component $h$ :

$$
q^{h}(z) f^{Z}(z \mid \boldsymbol{\theta})=\lambda_{h}\left(z \mid \theta_{h}\right) \exp \left(-\int_{0}^{Z} \lambda^{H}(\chi \mid \boldsymbol{\theta}) d \chi\right)
$$

$\boldsymbol{\theta} \oplus z$ denotes addition of a scalar $z$ to each of the coordinates of vector $\boldsymbol{\theta}$, i.e. $\boldsymbol{\theta} \oplus z=$ $\left(\theta_{1}+z, \theta_{2}+z, \ldots, \theta_{n_{2}}+z\right)$, and $(\boldsymbol{\theta} \oplus z)^{\left(0_{h}\right)}$ means the $h^{\text {th }}$ coordinate of vector $\boldsymbol{\theta} \oplus z$ is replaced by zero, i.e. $(\boldsymbol{\theta} \oplus z)^{\left(0_{h}\right)}=\left(\theta_{1}+z, \ldots, \theta_{h-1}+z, 0, \theta_{h+1}+z, \ldots, \theta_{n_{2}}+z\right)$.

Now, let us extend the Equation (C.1) to obtain the expected value of a random variable of interest over $[0, i \tau]$ with the periodic inspections being performed at times $\tau, 2 \tau, \ldots, i \tau$ and $i=$ $2, \ldots, l$ :

$G_{i, s}\left(\tau, t_{s}, \boldsymbol{\theta}, \zeta^{*}, m_{c}, m_{s}\right)$

$=\int_{0}^{\tau} \int_{0}^{z} \sum_{h=1}^{m_{2}}\left\{\left(\left[\psi(y, z, 0)+G_{i, s}\left(\tau-z, t_{s}+y, \boldsymbol{\theta} \oplus z, \zeta^{*}, m_{c}+1, m_{s}\right)\right] r_{s}^{Y}\left(m_{c}, m_{s}\right)\right.\right.$

$\left.+\left[\psi(y, z, 1)+G_{i, s}\left(\tau-z, 0, \boldsymbol{\theta} \oplus z, \zeta^{*}, 0, m_{s}\right)\right] \bar{r}_{s}^{Y}\left(m_{c}, m_{s}\right)\right) r_{h}^{Z}\left(\theta_{h}+z, \zeta_{h}^{*}\right)$

$+\left(\left[\psi(y, z, 0)+G_{i, s}\left(\tau-z, t_{s}+x,(\boldsymbol{\theta} \oplus z)^{\left(0_{h}\right)}, \zeta^{*}, m_{c}+1, m_{s}\right)\right] r_{s}^{X}\left(m_{c}, m_{s}\right)\right.$

$\left.+\left[\psi(y, z, 1)+G_{i, s}\left(\tau-z, 0,(\boldsymbol{\theta} \oplus z)^{\left(0_{h}\right)}, \zeta^{*}, 0, m_{s}\right)\right] \bar{r}_{s}^{X}\left(m_{c}, m_{s}\right)\right) \bar{r}_{h}^{Z}\left(\theta_{h}\right.$

$\left.\left.+z, \zeta_{h}^{*}\right)\right\} q^{h}(z) f^{Y}\left(y \mid t_{s}\right) f^{Z}(z \mid \boldsymbol{\theta}) d y d z$

$+\int_{0}^{\tau} \sum_{s=1}^{m_{1}}\left\{\left[\psi(z, z, 2)+G_{i, s}\left(\tau-z, t_{j}+z, \boldsymbol{\theta} \oplus z, \zeta^{*}, m_{c}, m_{s}\right)\right] r_{h}^{Z}\left(\theta_{h}+z, \zeta_{h}^{*}\right)\right.$

$+\left[\psi(z, z, 2)+G_{i, s}\left(\tau-z, t_{j}+z,(\boldsymbol{\theta} \oplus z)^{\left(0_{m}\right)}, \zeta^{*}, m_{c}, m_{s}\right)\right] \bar{r}_{h}^{Z}\left(\theta_{h}\right.$

$\left.\left.+z, \zeta_{h}^{*}\right)\right\} q^{h}(z) \mathcal{R}^{Y}\left(z \mid t_{s}\right) f^{Z}(z \mid \boldsymbol{\theta}) d z$

$+\int_{0}^{\tau}\left[\left(\psi(y, \tau, 0)+G_{i-1, s}\left(\tau, t_{s}+x, \boldsymbol{\theta} \oplus \tau, \zeta^{*}, m_{c}+1, m_{s}\right)\right) r_{s}^{Y}\left(m_{c}, m_{s}\right)\right.$

$\left.+\left(\psi(y, \tau, 1)+G_{i-1, s}\left(\tau, 0, \boldsymbol{\theta} \oplus \tau, \zeta^{*}, 0, m_{s}\right)\right) \bar{r}_{j}^{X}\left(m_{c}, m_{s}\right)\right] f^{Y}\left(y \mid t_{s}\right) d y \mathcal{R}^{Z}(\sigma \mid \boldsymbol{\theta})$ 
$+\left(\psi(\tau, \tau, 2)+G_{i-1, s}\left(\tau, t_{j}+\tau, \boldsymbol{\theta} \oplus \tau, \zeta^{*}, m_{c}, m_{s}\right)\right) \mathcal{R}^{Y}\left(\tau \mid t_{s}\right) \mathcal{R}^{Z}(\tau \mid \boldsymbol{\theta})$.

To obtain the required expected values in Equation (22), $G_{i, s}\left(\tau, t_{s}, \boldsymbol{\theta}, \zeta^{*}, m_{c}, m_{s}\right)$ should be obtained for $i=1, \ldots, l$ for a given $m_{s}$, assuming $m_{c}=0$ at time zero. The integrals in Equations (B.1) and (B.2) can be approximated using Simpson's rule. However, due to recursive nature of the equations and discretisation approach of Simpson's rule, a system of equations needed to be solved to obtain the expected values, which makes the procedure computationally intensive with less accurate results.

Similarly to Equations (B.1) and (B.2) for Stage 2, recursive formula can be derived to obtain the required expected values in Equation (27) in Stage 3. The equations are similar to (B.1) and (B.2), but the difference is that $m_{s}$ is substituted by $m_{s}^{*}$, since in Stage 3, the current number of minimal repairs is compared with the optimal number of minimal repairs $m_{S}^{*}$ obtained from Stage 2 .

In Model 4, in addition to the expected values for the number of minimal repairs, replacements and expected uptime of soft-type components, we need to obtain the expected numbers of minimal repairs, corrective and preventive replacements of hard-type components, as well as the expected number of opportunistic inspections (see Equation (29)). Thus, we need to incorporate the preventive replacements of hard-type components at periodic inspections, assuming $m_{c}=0$ at time zero:

$$
\begin{aligned}
& G_{i, s}\left(\tau, t_{s}, \boldsymbol{\theta}, \zeta^{*}, m_{c}, m_{s}\right) \\
& =\int_{0}^{\tau} \int_{0}^{z} \sum_{h=1}^{m_{2}}\left\{\left(\left[\psi(y, z, 0)+G_{i, s}\left(\tau-z, t_{s}+y, \boldsymbol{\theta} \oplus z, \zeta^{*}, m_{c}+1, m_{s}\right)\right] r_{s}^{Y}\left(m_{c}, m_{s}\right)\right.\right. \\
& \left.+\left[\psi(y, z, 1)+G_{i, s}\left(\tau-z, 0, \boldsymbol{\theta} \oplus z, \zeta^{*}, 0, m_{s}\right)\right] \bar{r}_{s}^{Y}\left(m_{c}, m_{s}\right)\right) r_{h}^{Z}\left(\theta_{h}+z, \zeta_{h}^{*}\right) \\
& +\left(\left[\psi(y, z, 0)+G_{i, s}\left(\tau-z, t_{s}+y,(\boldsymbol{\theta} \oplus z)^{\left(0_{h}\right)}, \zeta^{*}, m_{c}+1, m_{s}\right)\right] r_{s}^{Y}\left(m_{c}, m_{s}\right)\right. \\
& \left.+\left[\psi(y, z, 1)+G_{i, s}\left(\tau-z, 0,(\boldsymbol{\theta} \oplus z)^{\left(0_{h}\right)}, \zeta^{*}, 0, m_{s}\right)\right] \bar{r}_{s}^{Y}\left(m_{c}, m_{s}\right)\right) \bar{r}_{h}^{Z}\left(\theta_{h}\right. \\
& \left.\left.+z, \zeta_{h}^{*}\right)\right\} q^{h}(z) f^{Y}\left(y \mid t_{s}\right) f^{Z}(z \mid \boldsymbol{\theta}) d y d z
\end{aligned}
$$




$$
\begin{aligned}
& +\int_{0}^{\tau} \sum_{h=1}^{m_{1}}\left\{\left[\psi(z, z, 2)+G_{i, s}\left(\tau-z, t_{s}+y, \boldsymbol{\theta} \oplus z, \zeta^{*}, m_{c}, m_{s}\right)\right] r_{h}^{Z}\left(\theta_{h}+z, \zeta_{h}^{*}\right)\right. \\
& +\left[\psi(z, z, 2)+G_{i, s}\left(\tau-z, t_{s}+z,(\boldsymbol{\theta} \oplus z)^{\left(0_{h}\right)}, \zeta^{*}, m_{c}, m_{s}\right)\right] \bar{r}_{h}^{Z}\left(\theta_{h}\right. \\
& \left.\left.+z, \zeta_{h}^{*}\right)\right\} q^{h}(z) \mathcal{R}^{Y}\left(z \mid t_{s}\right) f^{Z}(z \mid \boldsymbol{\theta}) d z \\
& +\int_{0}^{\tau}\left[\left(\psi(y, \tau, 0)+G_{i-1, s}\left(\tau, t_{s}+y, \boldsymbol{\vartheta}\left(\boldsymbol{\theta} \oplus \tau, \zeta^{*}\right), \zeta^{*}, m_{c}+1, m_{s}\right)\right) r_{j}^{X}\left(m_{c}, m_{s}\right)\right. \\
& \left.+\left(\psi(y, \tau, 1)+G_{i-1, s}\left(\tau, 0, \boldsymbol{\vartheta}\left(\boldsymbol{\theta} \oplus \tau, \boldsymbol{\zeta}^{*}\right), \zeta^{*}, 0, m_{s}\right)\right) \bar{r}_{s}^{Y}\left(m_{c}, m_{s}\right)\right] f^{Y}\left(y \mid t_{s}\right) d y \mathcal{R}^{Z}(\sigma \mid \boldsymbol{\theta}) \\
& +\left(\psi(\tau, \tau, 2)+G_{i-1, s}\left(\tau, t_{s}+\tau, \boldsymbol{\vartheta}\left(\boldsymbol{\theta} \oplus \tau, \zeta^{*}\right), \zeta^{*}, m_{c}, m_{s}\right)\right) \mathcal{R}^{Y}\left(\tau \mid t_{s}\right) \mathcal{R}^{Z}(\tau \mid \boldsymbol{\theta}),
\end{aligned}
$$

where $\boldsymbol{\vartheta}\left(\boldsymbol{\theta}, \boldsymbol{\zeta}^{*}\right)=\left(\vartheta_{1}, \ldots, \vartheta_{n_{2}}\right)$ and $\vartheta_{h}$ is defined as follows:

$$
\vartheta_{h}=\left\{\begin{array}{cc}
\theta_{h}, & \text { if } \theta_{h} \leq \zeta_{h}^{*} \\
0, & \text { otherwise }
\end{array}, \quad h=1,2, \ldots, n_{2} .\right.
$$

If $f_{h}^{Z}\left(z \mid \theta_{h}\right)$ and $\mathcal{R}_{h}^{Z}\left(z \mid \theta_{h}\right)$ are the density and reliability functions of hard-type component $h$, then the recursive formula for the expected values of interest, i.e. corrective and preventive replacement of hard-type components, as well as the expected number of opportunistic inspections for hard-type components are derived as follows:

$$
\begin{aligned}
& G_{1, h}^{H}\left(\tau, \theta_{h}, \zeta_{h}^{*}\right)=\int_{0}^{\tau}\left\{\left[\psi(z, 0, \tau)+G_{1, h}^{H}\left(\tau-z, \theta_{h}+z, \zeta_{h}^{*}\right)\right] r_{h}^{Z}\left(\theta_{h}+z, \zeta_{h}^{*}\right)\right. \\
& \left.+\left[\psi(z, 1, \tau)+G_{1, h}^{H}\left(\tau-z, 0, \zeta_{h}^{*}\right)\right] \bar{r}_{h}^{Z}\left(\theta_{h}+z, \zeta_{h}^{*}\right)\right\} f_{h}^{Z}\left(z \mid \theta_{h}\right) d z \\
& +\mathcal{R}_{h}^{Z}\left(\tau \mid \theta_{h}\right)\left[\psi(\tau, 0, \tau) r_{h}^{Z}\left(\theta_{h}+\tau, \zeta_{h}^{*}\right)+\psi(\tau, 1, \tau) \bar{r}_{h}^{Z}\left(\theta_{h}+\tau, \zeta_{h}^{*}\right)\right],
\end{aligned}
$$

and

$$
\begin{aligned}
& G_{i, h}^{H}\left(\tau, \theta_{h}, \zeta_{h}^{*}\right)=\int_{0}^{\tau}\left\{\left[\psi(z, 0, \tau)+G_{i, h}^{H}\left(\tau-z, \theta_{h}+z, \zeta_{h}^{*}\right)\right] r_{h}^{Z}\left(\theta_{h}+z, \zeta_{h}^{*}\right)\right. \\
& \left.+\left[\psi(z, 1, \tau)+G_{i, h}^{H}\left(\tau-z, 0, \zeta_{h}^{*}\right)\right] \bar{r}_{h}^{Z}\left(\theta_{h}+z, \zeta_{h}^{*}\right)\right\} f_{h}^{Z}\left(z \mid \theta_{h}\right) d z \\
& +\left\{\left[\psi(\tau, 0, \tau)+G_{i-1, h}^{H}\left(\tau, \theta_{h}+\tau, \zeta_{h}^{*}\right)\right] r_{h}^{Z}\left(\theta_{h}+\tau, \zeta_{h}^{*}\right)\right. \\
& \left.+\left[\psi(\tau, 1, \tau)+G_{i-1, h}^{H}\left(\tau, 0, \zeta_{h}^{*}\right)\right] \bar{r}_{h}^{Z}\left(\theta_{h}+\tau, \zeta_{h}^{*}\right)\right\} \mathcal{R}_{h}^{Z}\left(\tau \mid \theta_{h}\right)
\end{aligned}
$$


For the number of minimal repairs of a hard-type component inside scheduled inspection intervals:

$$
\psi\left(z, I_{h}, \tau\right)=\left\{\begin{array}{ll}
1, & z<\tau \\
0, & \text { otherwise }
\end{array} \quad I_{h}=0 .\right.
$$

For the number of corrective replacements of a hard-type component inside scheduled inspection intervals:

$$
\psi\left(z, I_{h}, \tau\right)=\left\{\begin{array}{ll}
1, & z<\tau \\
0, & \text { otherwise }
\end{array} \quad I_{h}=1 .\right.
$$

For the number of preventive replacements of a hard-type component:

$$
\psi\left(z, I_{h}, \tau\right)=\left\{\begin{array}{ll}
1, & z<\tau \\
0, & \text { otherwise }
\end{array} \quad I_{h}=1 .\right.
$$

Since two failures cannot take place at the same time, the expected number of opportunistic inspections is the sum of the expected number of minimal repairs and the expected number of corrective replacements for all hard-type components. 


\section{Appendix C. Simulation flowcharts for the case of corrective maintenance of hard- type components and opportunistic inspection of soft-type components}

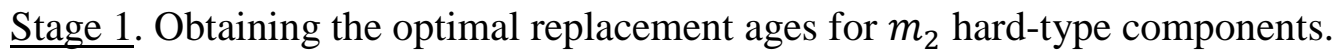

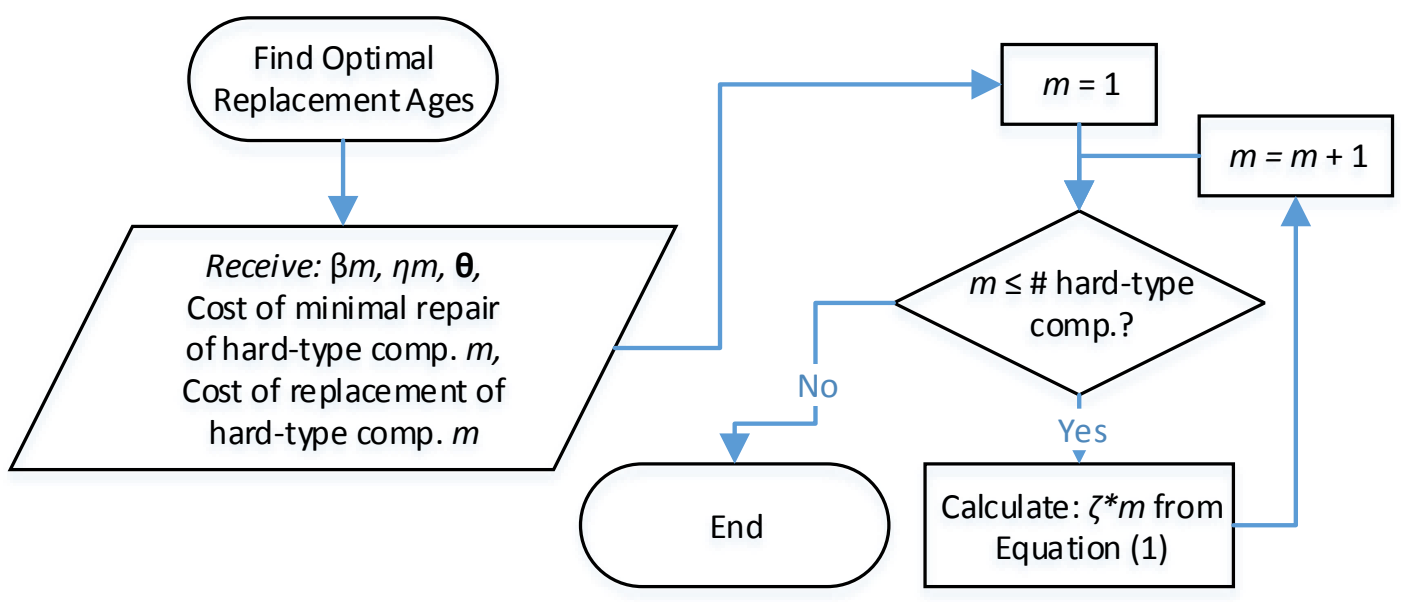

Figure C. 1: Flowchart for Stage 1 of Model 3.

Stage 2. Obtaining the optimal number of minimal repairs before replacement for a given soft-type component $j$, inspection period $\tau$ and cycle length $\omega$. 


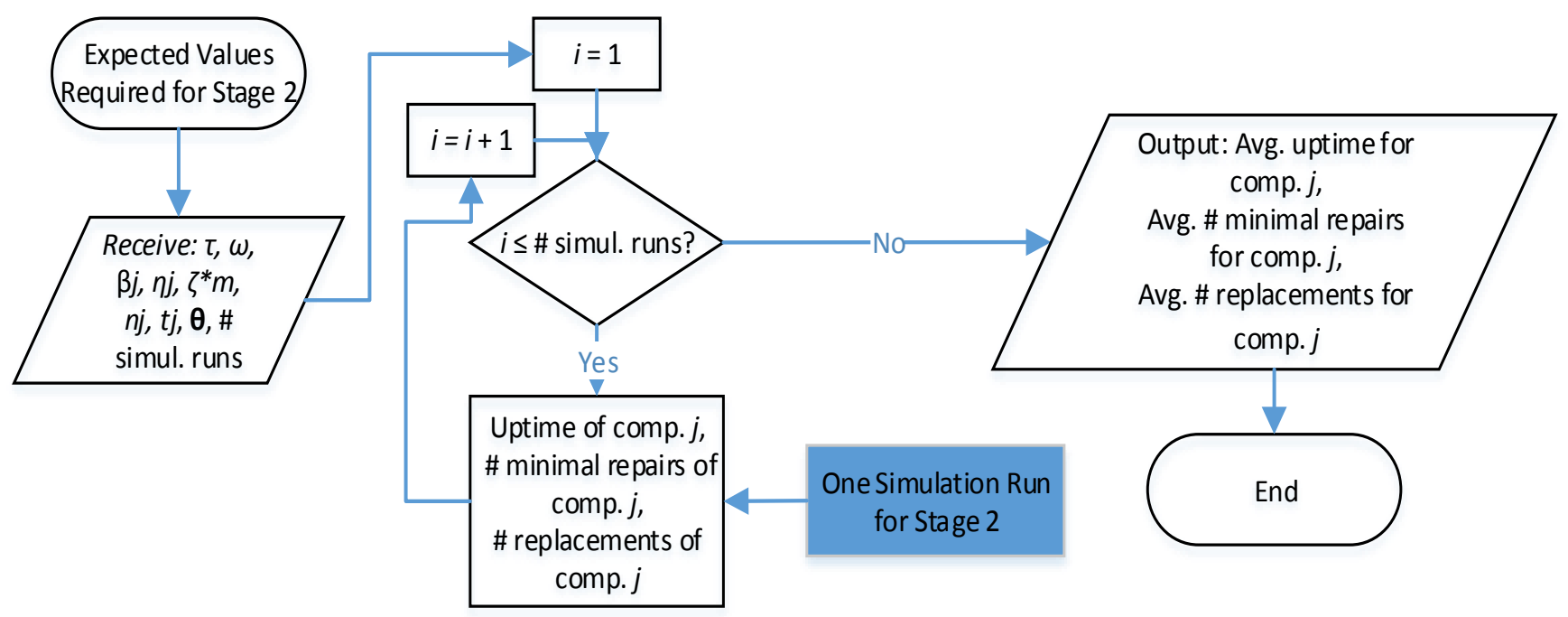

Figure C. 2: Flowchart for obtaining expected values required for Stage 2 of Model 3.

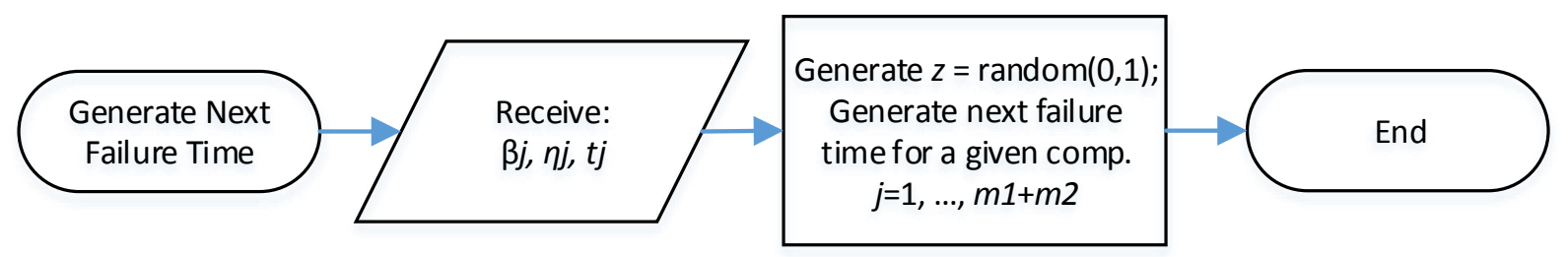

Figure C. 3: Flowchart for generating failure times for Stage 2 of Model 3. 


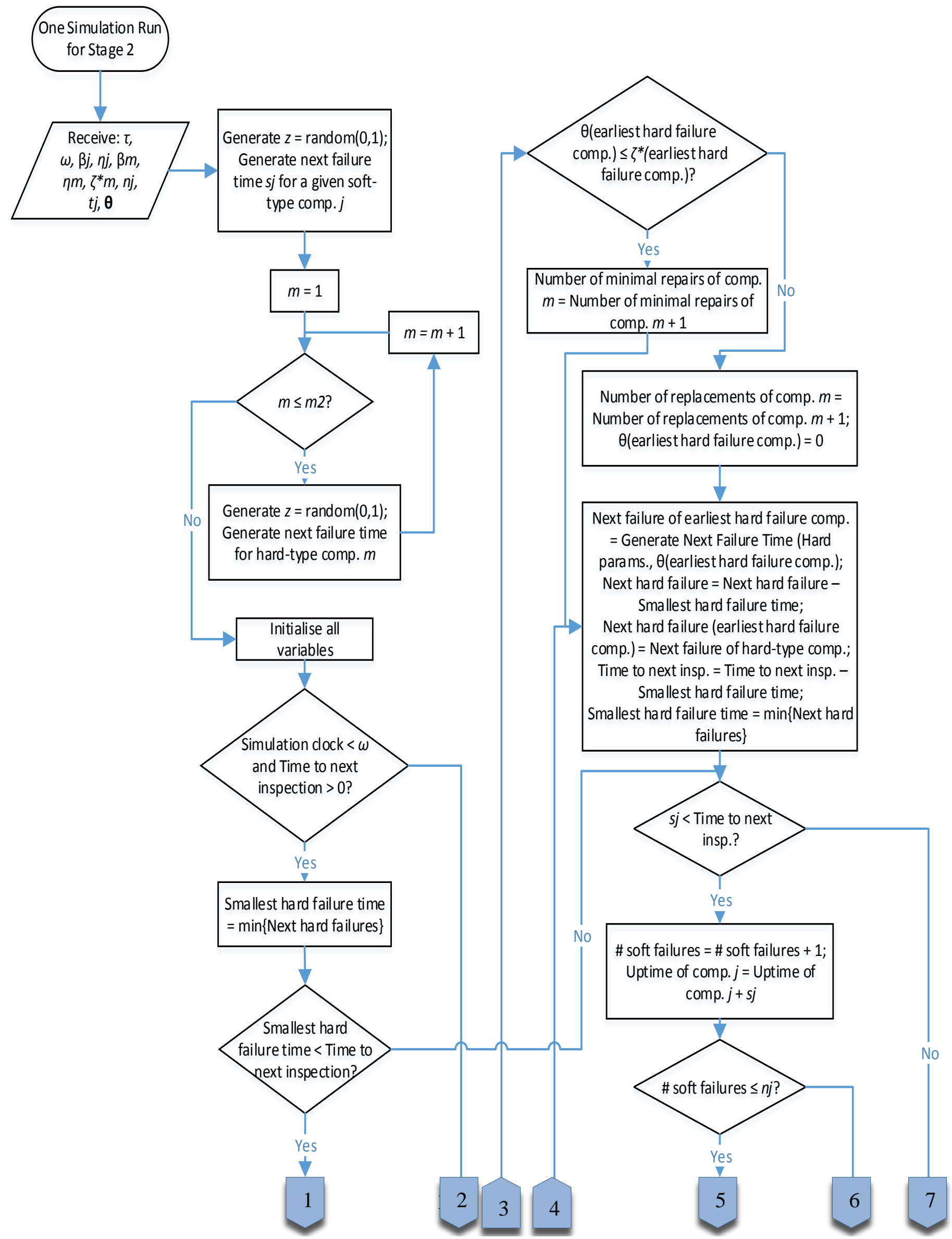

Figure C. 4: Flowchart for performing 1 simulation run for Stage 2 of Model 3. 


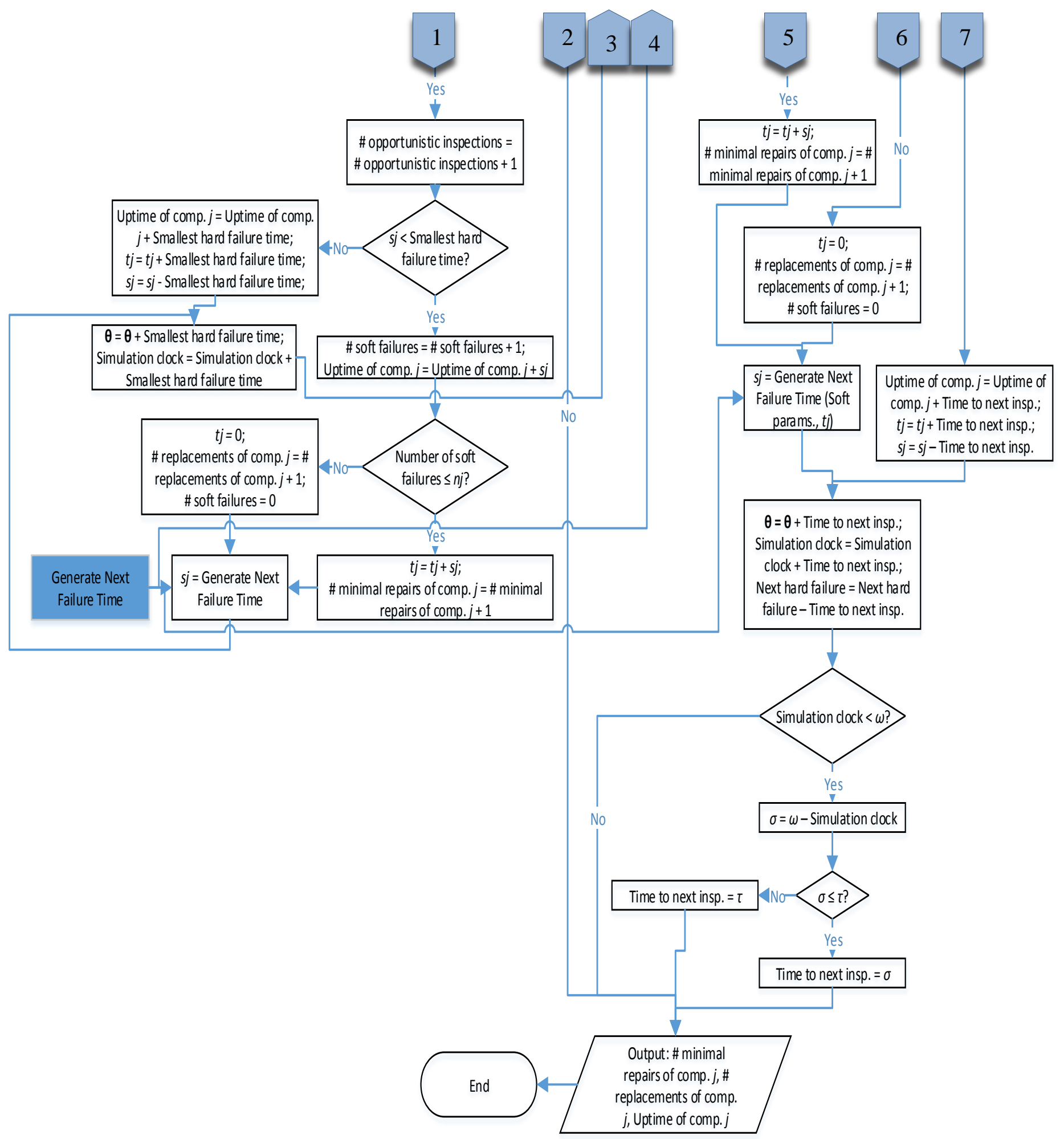

Figure C. 5: Flowchart for performing 1 simulation run for Stage 2 of Model 3 (Continued). 


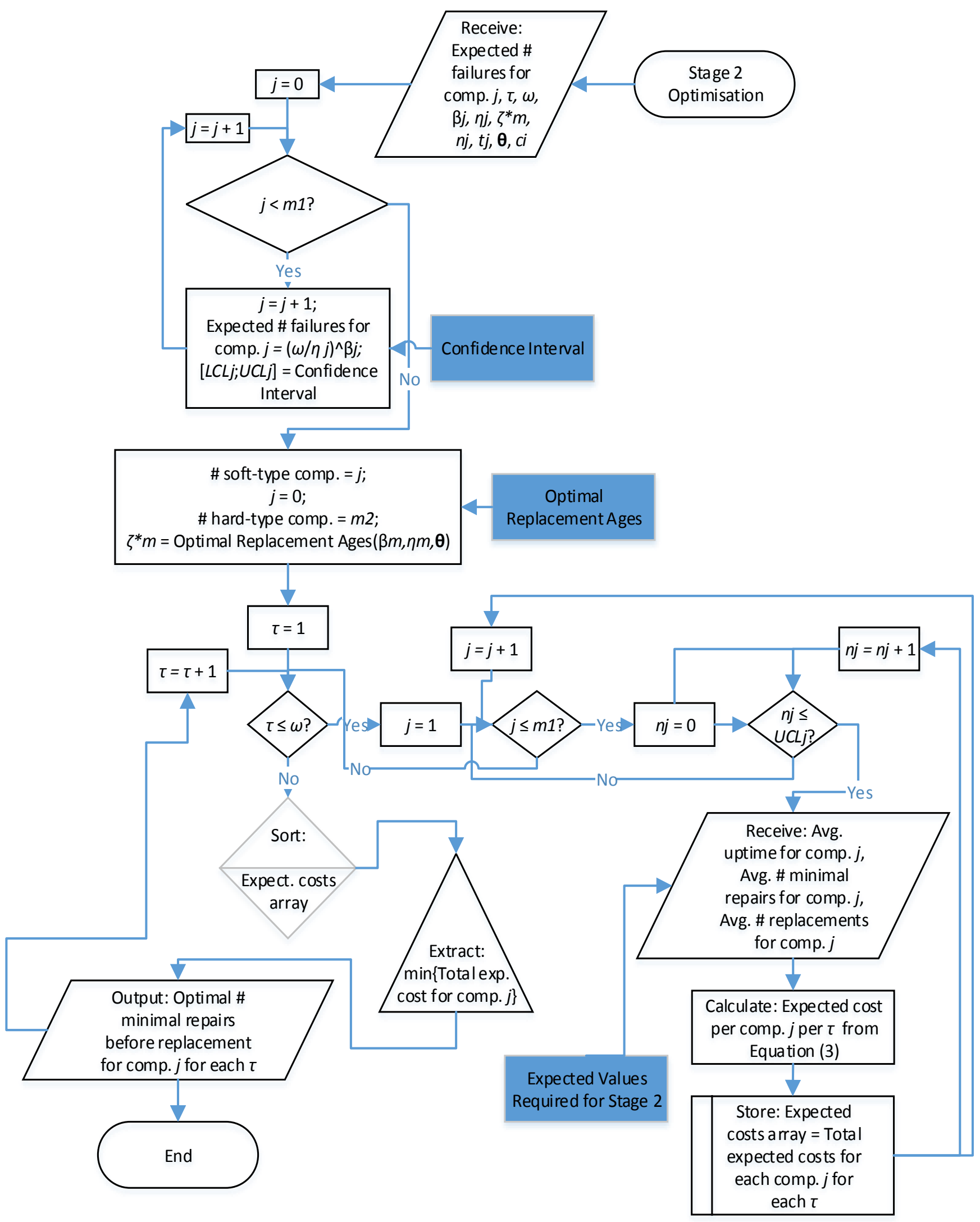

Figure C. 6: Flowchart for performing Stage 2 optimisation of Model 3. 


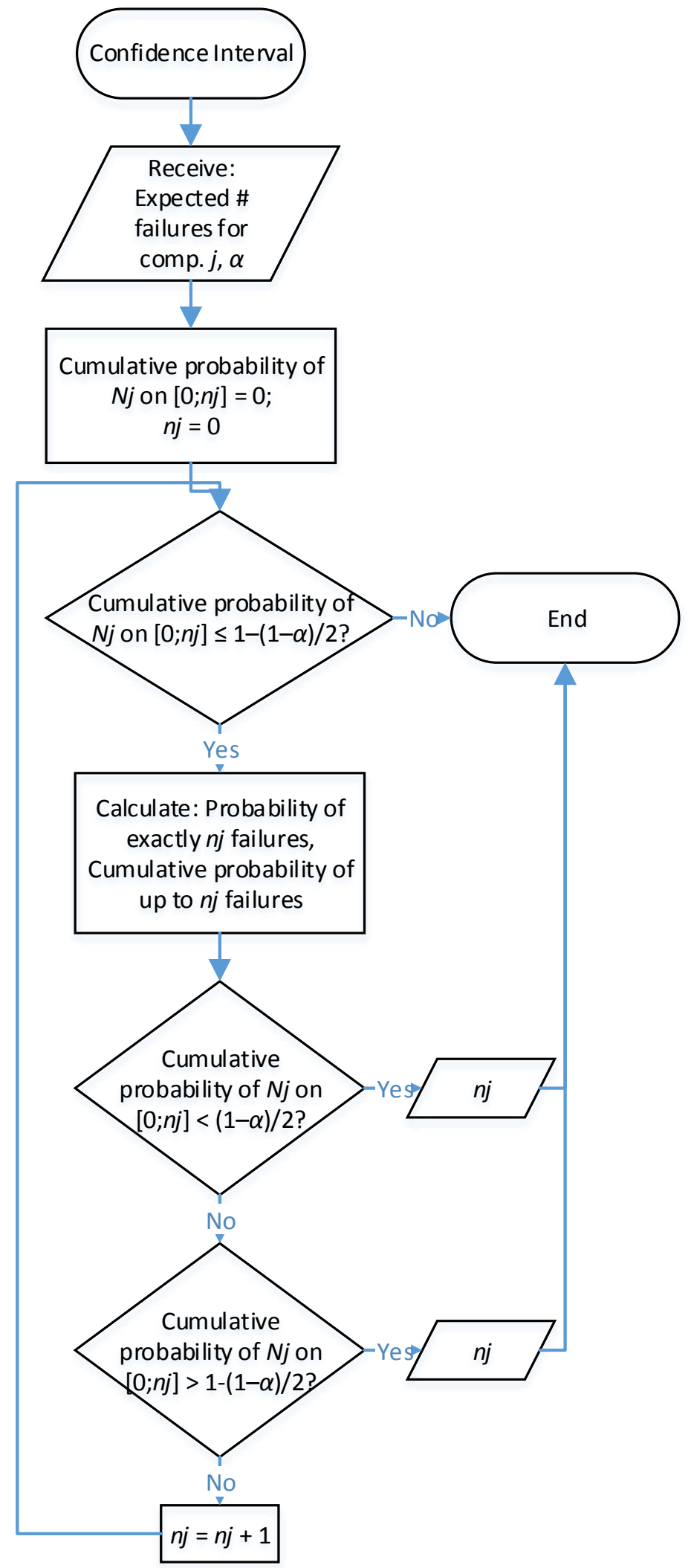

Figure C. 7: Flowchart for calculating confidence interval on the expected number of softtype component failures for Stage 2 of Model 3. 


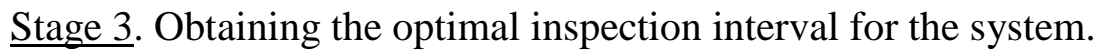

At this stage, since the soft-type components' age at failure is unknown, $M_{l, j}\left(\tau, t_{j}, \boldsymbol{\theta}, \zeta^{*}, n_{C}, n_{j}^{*}\right), \quad R_{l, j}\left(\tau, t_{j}, \boldsymbol{\theta}, \zeta^{*}, n_{C}, n_{j}^{*}\right)$ and $U_{l, j}\left(\tau, t_{j}, \boldsymbol{\theta}, \boldsymbol{\zeta}^{*}, n_{C}, n_{j}^{*}\right)$ are obtained numerically from simulation for soft-type component $j=1, \ldots, m_{1}$. For brevity of notation, we further denote these functions $M_{l, j, n_{j}^{*}}, R_{l, j, n_{j}^{*}}$ and $U_{l, j, n_{j}^{*}}$, correspondingly. Function "One Simulation Run for Stage $3\left(\tau, \omega, \beta_{j}, \eta_{j}, \beta_{m}, \eta_{m}, \zeta_{m}^{*}, n_{j}, t_{j}, \boldsymbol{\theta}\right)$ " differs from function "One Simulation Run for Stage $2\left(\tau, \omega, \beta_{j}, \eta_{j}, \beta_{m}, \eta_{m}, \zeta_{m}^{*}, n_{j}, t_{j}, \boldsymbol{\theta}\right)$ "' in that the latter calculates the optimal number of minimal repairs before replacement marginally for each soft-type component, while the former calculates the optimal periodic inspection interval for the entire system based on the optimal number of minimal repairs before replacement. These expected values are then used for calculating $E\left[C_{S, \tau}^{T}\right]$ for $\tau=1, \ldots, \omega$, which is then minimised to find the optimal inspection interval $\tau^{*}$. 


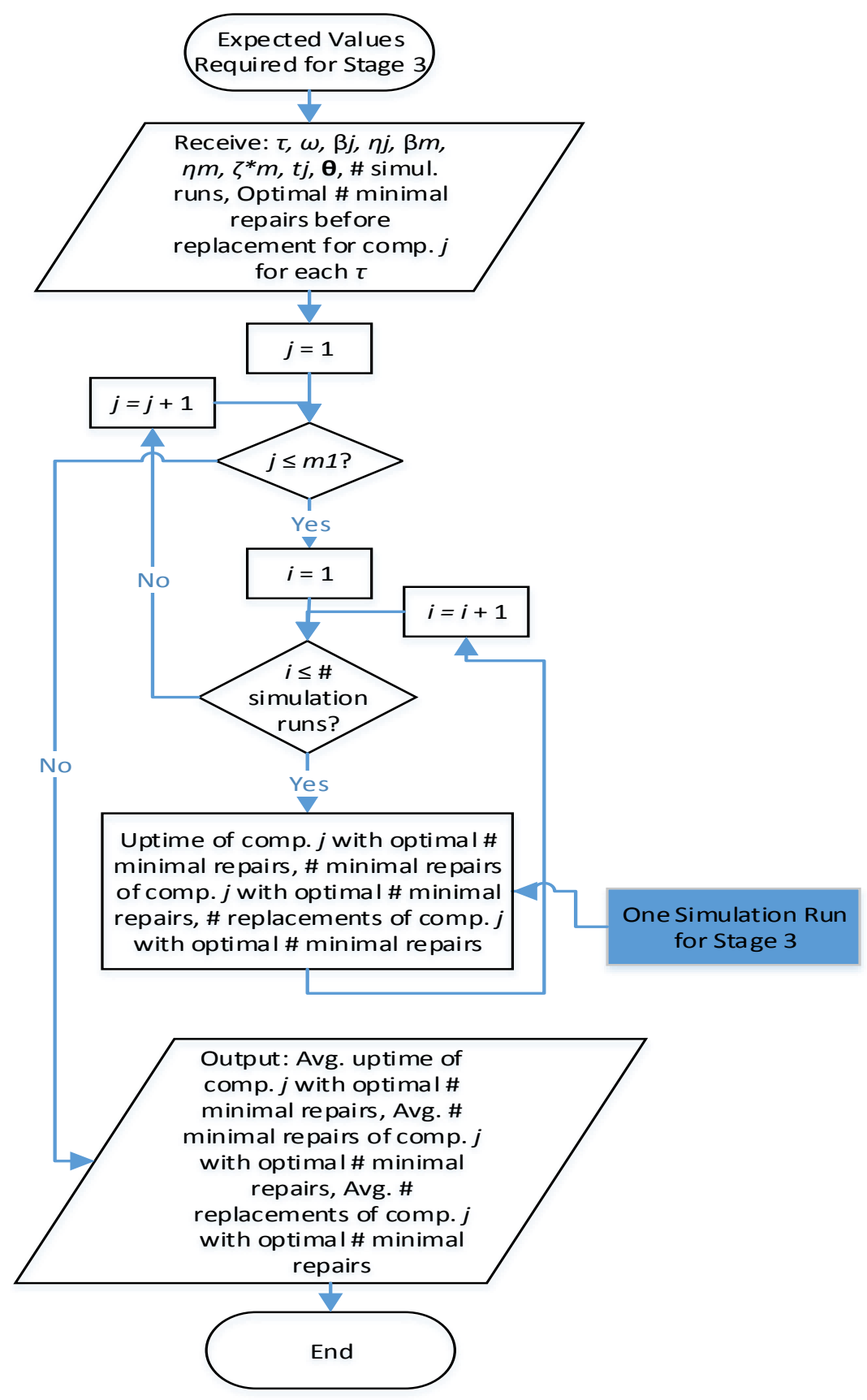

Figure C. 8: Flowchart for calculating the expected values for Stage 3 of Model 3. 


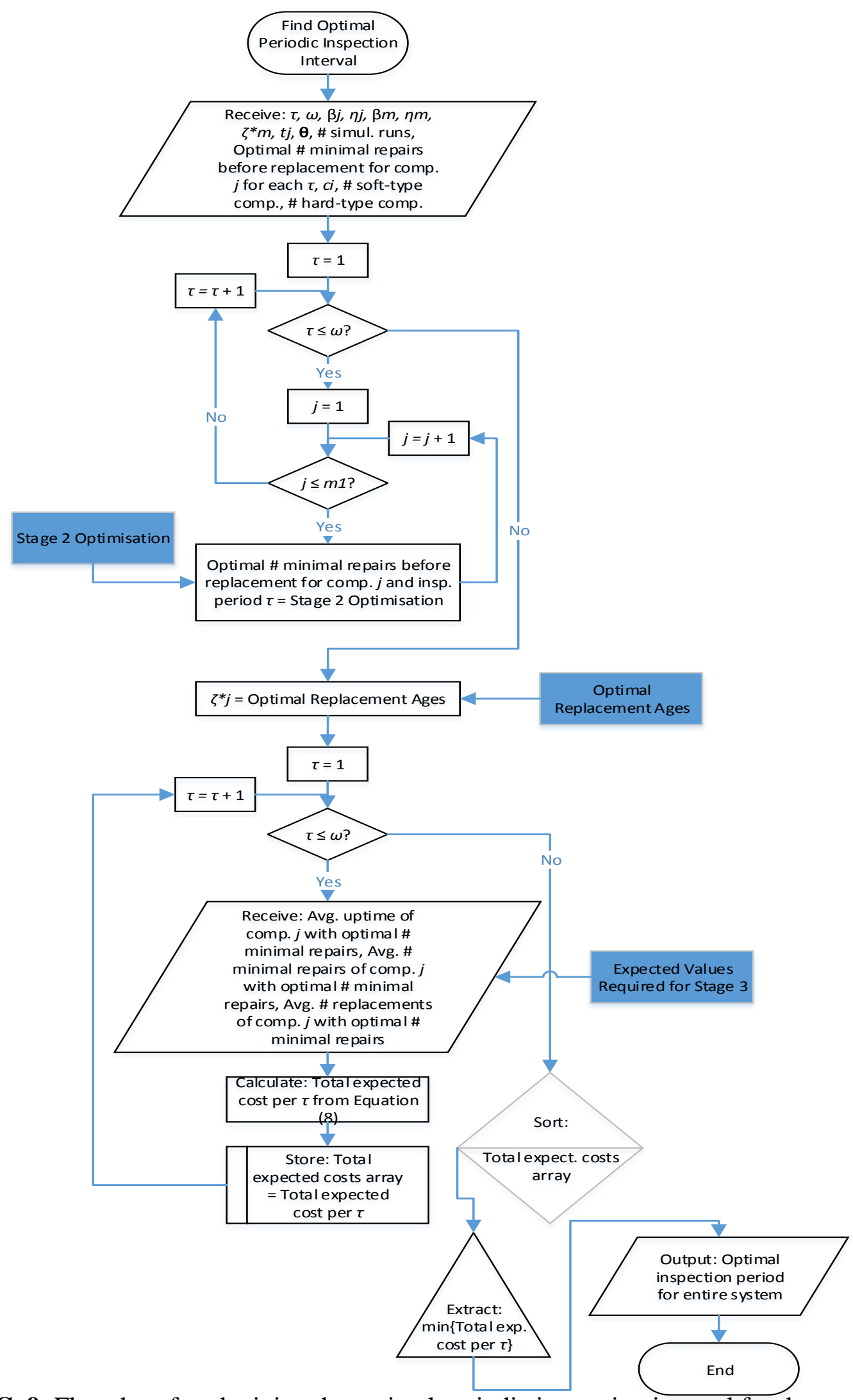

Figure C. 9: Flowchart for obtaining the optimal periodic inspection interval for the system in Stage 3 of Model 3. 


\section{References}

[1] H. Wang, "A survey of maintenance policies of deteriorating systems," European Journal of Operational Research, vol. 139, pp. 469-489, 2002.

[2] D. I. Cho and M. Parlar, "A survey of maintenance models for multi-unit systems," European Journal of Operational Research, vol. 51, pp. 1-23, 1991.

[3] G. J. Wang and Y. L. Zhang, "A Bivariate Mixed Policy for a Simple Repairable System Based on Preventive Repair and Failure Repair," Applied Mathematical Modelling, vol. 33, pp. 3354-3359, 2009.

[4] Y. L. Zhang and G. J. Wang, "A geometric process repair model for a series repairable system with k dissimilar components," Applied Mathematical Modelling, vol. 31, pp. 1997-2007, 2007.

[5] Q. Wu and J. Zhang, "A bivariate replacement policy for a cold standby system under Poisson shocks," American Journal of Mathematical and Management Sciences, vol. 32, pp. 145-177, 2013.

[6] V. H. Coria, S. Maximov, F. Rivas-Davalos, C. L. Melchor and J. L. Guardado, "Analytical method for optimization of maintenance policy based on available system failure data," Reliability Engineering and System Safety, vol. 135, pp. 55-63, 2015.

[7] E.-S. Pan, W.-Z. Liao and M.-L. Zhuo, "Periodic preventive maintenance policy with infinite time and limit of reliability based on health index," J. Shanghai Jiaotong Univ. (Sci.), vol. 15, no. 2, pp. 231-235, 2010.

[8] S. Taghipour and D. Banjevic, "Periodic Inspection Optimization Models for a Repairable System subject to Hidden Failures," IEEE Transactions on Reliability, vol. 60, no. 1, pp. 275-285, 2011.

[9] S. Taghipour, "Optimal inspection model for a load-sharing redundant system," Proceedings of the Annual Reliability and Maintainability Symposium, pp. 198-202, 2014.

[10] M. L. Kassaei and S. Taghipour, "Inspection optimization model for a k-out-of-n loadsharing system with dependent components," Proceedings of the IIE Annual Conference \& Expo, vol. 5, pp. 3723-3731, 2013.

[11] S. Taghipour and M. L. Kassaei, "Periodic Inspection Optimization of a k-out-of-n LoadSharing System," IEEE Transactions on Reliability, vol. 64, no. 3, pp. 1116-1127, 2015.

[12] X.-J. Zhou, Z.-Q. Lu, L.-F. Xi and J. Lee, "Opportunistic preventive maintenance optimization for multi-unit series systems with combing multi-preventive maintenance techniques," Journal of Shanghai Jiaotong University (Science), vol. 15, pp. 513-518, 2010.

[13] K.-H. Wang and C.-C. Kuo, "Cost and probabilistic analysis of series systems with mixed standby components," Applied Mathematical Modelling, vol. 24, pp. 957-967, 2000.

[14] S. Taghipour and D. Banjevic, "Optimum inspection interval for a system under periodic and opportunistic inspections," IIE Transactions, vol. 44, pp. 932-948, 2012. 
[15] S. Taghipour and D. Banjevic, "Optimal inspection of a complex system subject to periodic and opportunistic inspections and preventive replacements," European Journal of Operational Research, vol. 220, no. 3, pp. 649-660, 2012.

[16] B. Lienhardt, E. Hugues, C. Bes and D. Noll, "Failure-Finding Frequency for a Repairable System Subject to Hidden Failures," Journal of Aircraft, vol. 45, no. 5, pp. 1804-1809, September-October 2008.

[17] S.-H. Sheu, Y.-L. Chen, C.-C. Chang and Z.-G. Zhang, "Extended optimal replacement policy for a system subject to non-homogeneous pure birth shocks," Computers \& Industrial Engineering, vol. 64, p. 573-579, 2013.

[18] T. Nakagawa, M. Motoori and K. Yasui, "Optimal Testing Policy for a Computer System with Intermittent Faults," Reliability Engineering and System Safety, vol. 27, pp. 213218, 1990.

[19] E. T. S. Bjarnason, S. Taghipour and D. Banjevic, "Joint optimal inspection and inventory for a k-out-of-n system," Reliability Engineering and System Safety, vol. 131, pp. 203-215, 2014.

[20] E. T. S. Bjarnason, S. Taghipour, D. Banjevic and A. K. S. Jardine, "Joint Optimization of Periodic Inspection and Inventory for a k-out-of-n System," Proceedings of the IIE Annual Conference \& Expo 2013, vol. 5, pp. 3632-3641, 2013.

[21] E. T. S. Bjarnason and S. Taghipour, "Periodic Inspection Frequency and Inventory Policies for a k-out-of-n System," IIE Transactions, vol. 48, no. 7, pp. 638-650, 2016.

[22] N. Fard and X. Zheng, "An Approximate Method for Non-Repairable Systems Based on Opportunistic Replacement Policy," Reliability Engineering and System Safety, vol. 33, no. 2, pp. 277-288, 1991.

[23] V. Makis and A. K. S. Jadine, "Optimal Replacement Policy for a General Model with Imperfect Repair," The Journal of the Operational Research Society, vol. 43, no. 2, pp. 111-120, 1992.

[24] S. Taghipour, D. Banjevic and A. K. S. Jardine, "Periodic Inspection Optimization Model for a Complex Repairable System," Reliability Engineering and System Safety, vol. 95, no. 9, pp. 944-952, 2010.

[25] Y. H. Chien and S. H. Sheu, "Extended optimal age-replacement policy with minimal repair of a system subject to shocks," European Journal of Operational Research, vol. 174, no. 1, pp. 169-181, 2006.

[26] S.-H. Sheu, S.-H. Li and C.-C. Chang, "A generalised maintenance policy with agedependent minimal repair cost for a system subject to shocks under periodic overhaul," International Journal of Systems Science, vol. 43, no. 6, pp. 1007-1013, June 2012.

[27] S.-H. Sheu and W. S. Griffith, "Optimal age-replacement policy with age-dependent minimal-repair and random-leadtime," IEEE Transactions on Reliability, vol. 50, no. 3, pp. 302-309, 2001.

[28] T. Nowakowski and S. Werbinka, "On Problems of Multicomponent System Maintenance Modelling," International Journal of Automation and Computing, vol. 6, no. 4, pp. 364-378, November 2009.

[29] Y. L. Zhang and S. Wu, "Reliability analysis for a $\mathrm{k} / \mathrm{n}(\mathrm{F})$ system with repairable repairequipment," Applied Mathematical Modelling, vol. 33, pp. 3052-3067, 2009. 
[30] K. S. Park, "Optimal Number of Minimal Repairs before Replacement," IEEE Transactions on Reliability, Vols. R-28, no. 2, pp. 137-140, June 1979.

[31] S. Ozekici, "Optimal periodic replacement of multicomponent reliability systems," Operations Research, vol. 36, no. 4, pp. 542-552, 1988.

[32] X. Zhao, M. Fouladirad and C. Bérenguer, "Residual-based inspection/replacement policy for a deteriorating system with Markovian covariates," in Industrial Engineering and Engineering Management (IEEM), Macao, 2010.

[33] B. Su, "An optimal inspection and diagnosis policy for a multi-mode system," Reliability Engineering and System Safety, vol. 76, pp. 181-188, 2002.

[34] B. Castanier, A. Grall and C. Bérenguer, "A condition-based maintenance policy with non-periodic inspections for a two-unit series system," Reliability Engineering and System Safety, vol. 87, pp. 109-120, 2005.

[35] B. Castanier, C. Bérenguer and A. Grall, "A sequential condition-based repair/replacement policy with non-periodic inspections for a system subject to continuous wear," Appl. Stochastic Models Bus. Ind., vol. 19, p. 327-347, 2003.

[36] J. K. Vaurio, "Optimization of test and maintenance intervals based on risk and cost," Reliability Engineering and System Safety, vol. 49, no. 1, pp. 23-36, 1995.

[37] H. R. Golmakani and H. Moakedi, "Optimal non-periodic inspection scheme for a multicomponent repairable system using A* search algorithm," Computers \& Industrial Engineering, vol. 63, pp. 1038-1047, 2012.

[38] C. M. F. Lapa, C. M. N. A. Pereira and P. F. Frutuoso e Melo, "Surveillance test policy optimization through genetic algorithms using non-periodic intervention frequencies and considering seasonal constraints," Reliability Engineering and System Safety, vol. 81, pp. 103-109, 2003.

[39] J. S. Dagpunar, "A Maintenance Model with Opportunities and Interrupt Replacement Options," The Journal of the Operational Research Society, vol. 47, no. 11, pp. 14061409, 1996.

[40] W. Zhu, M. Fouladirad and C. Berenguer, "A reactive multi-component maintenance policy for offshore wind turbines," in European Safety and Reliability Conference (ESREL) 2013, Amsterdam, Netherlands, 2014.

[41] L. Cui and H. Li, "Opportunistic maintenance for multi-component shock models," Math. Meth. Oper. Res., vol. 63, pp. 493-511, 2006.

[42] T. Aven and R. Dekker, "A useful framework for optimal replacement models," Reliability Engineering and System Safety, vol. 58, no. 1, pp. 61-67, 1997.

[43] S. Taghipour and M. L. Kassaei, "Periodic Inspection Optimization of a k-out-of-n LoadSharing System," IEEE Transactions on Reliability, vol. 64, no. 3, pp. 1116-1127, 2015.

[44] T. K. Huynh, A. Barros and C. Bérenguer, "A Reliability-based Opportunistic Predictive Maintenance Model for k-out-of-n Deteriorating Systems," Chemical Engineering Transactions, vol. 33, pp. 493-498, 2013.

[45] K. T. Huynh, A. Barros and C. Bérenguer, "Multi-Level Decision-Making for the Predictive Maintenance of k-out-of-n:F Deteriorating Systems," IEEE Transactions on Reliability, vol. 64, no. 1, pp. 94-117, 2015. 
[46] R. E. Barlow and K. D. Heidtmann, "Computing k-out-of-n system reliability," IEEE Transactions on Reliability, Vols. R-33, no. 4, pp. 322-323, 1984.

[47] S. Taghipour, Reliability and Maintenance of Medical Devices, LAP Lambert Academic Publishing, 2012.

[48] S. Taghipour, D. Banjevic and A. K. S. Jardine, "Risk-based Inspection and Maintenance for Medical Equipment," Proceedings of the 2008 International Industrial Engineering Research Conference, pp. 104-109, 2008.

[49] R. E. Barlow and L. C. Hunter, "Optimum preventive maintenance policies," Operations Research, vol. 8, pp. 90-100, 1960.

[50] E. Gustavsson, M. Patriksson, A.-B. Strömberg, A. Wojciechowski and M. Önnheim, "Preventive maintenance scheduling of multi-component systems with interval costs," Computers \& Industrial Engineering, vol. 76, pp. 390-400, 2014.

[51] F. G. Badía, M. D. Berrade and C. A. Campos, "Optimal inspection and preventive maintenance of units with revealed and unrevealed failures," Reliability Engineering and System Safety, vol. 78, pp. 157-163, 2002.

[52] Y. Wang and H. Pham, "A Multi-Objective Optimization of Imperfect Preventive Maintenance Policy for Dependent Competing Risk Systems With Hidden Failure," IEEE Transactions on Reliability, vol. 60, no. 4, pp. 770-781, 2011.

[53] R. Laggoune, A. Chateauneuf and D. Aissani, "Opportunistic Policy for Optimal Preventive Maintenance of a Multi-Component System in Continuous Operating Units," Computers \& Chemical Engineering, vol. 33, pp. 1499-1510, 2009.

[54] P. P. Tambe, S. Mohite and M. S. Kulkarni, "Optimisation of opportunistic maintenance of a multi-component system considering the effect of failures on quality and production schedule: A case study," Int J Adv Manuf Technol, vol. 69, p. 1743-1756, 2013.

[55] K. S. Moghaddam, "Preventive maintenance and replacement optimization on CNC machine using multiobjective evolutionary algorithms," Int J Adv Manuf Technol, vol. 76, pp. 2131-2146, 2015.

[56] T. Lust, O. Roux and F. Riane, "Exact and heuristic methods for the selective maintenance problem," European Journal of Operational Research, vol. 197, pp. 11661177, 2009.

[57] R. Jamshidi and M. M. S. Esfahani, "Maintenance policy determination for a complex system consisting of series and cold standby system with multiple levels of maintenance action," Int J Adv Manuf Technol, vol. 78, p. 1337-1346, 2015.

[58] T. Dohi, A. Ashioka and S. Osaki, "Optimizing the repair-time limit replacement schedule with discounting and imperfect repair," Journal of Quality in Maintenance Engineering, vol. 7, no. 1, pp. 71-84, 2001.

[59] R. W. Drinkwater and N. A. J. Hastings, "An Economic Replacement Model," Operational Research Society, vol. 18, no. 2, pp. 121-138, 1967.

[60] P. K. Kapur and R. B. Garg, "Optimal Number of Minimal Repairs before Replacement with Repair Cost Limit," Reliability Engineering and System Safety, vol. 26, pp. 35-46, 1989. 
[61] Y. H. Chien, S. H. Sheu and C. C. Chang, "Optimal age-replacement time with minimal repair based on cumulative repair cost limit and random lead time," International Journal of Systems Science, vol. 40, no. 7, pp. 703-715, 2009.

[62] M. T. Lai, "A periodical replacement model based on cumulative repair-cost limit," Applied Stochastic Models in Business and Industry, vol. 23, no. 6, pp. 455-464, 2007.

[63] H. W. Block, W. S. Borges and T. H. Savits, "Age dependent minimal repair," Journal of Applied Probability, vol. 22, pp. 370-385, 1985.

[64] S. H. Sheu, Y. B. Lin and G. L. Liao, "Optimal policies with decreasing probability of imperfect maintenance," IEEE Transactions on Reliability, vol. 54, no. 2, pp. 347-357, 2005.

[65] S. H. Sheu, Y. B. Lin and G. L. Liao, "Optimum policies for a system with general imperfect maintenance," Reliability Engineering and System Safety, vol. 91, no. 3, pp. 362-369, 2006.

[66] S. H. Sheu and C. C. Chang, "An extended periodic imperfect preventive maintenance model with age-dependant failure type," IEEE Transactions on Reliability, vol. 58, no. 2, pp. 397-405, 2009.

[67] L. Cui, "Availability of a periodically inspected system with random repair or replacement times," Journal of Statistical Planning and Inference, vol. 131, no. 1, pp. 89-100, 2005.

[68] S. Okumura, "Determination of Inspection Schedules of Equipment by Variational Method," Mathematical Problems in Engineering, vol. 6, pp. 1-16, 2006.

[69] H. Pham and H. Wang, "Imperfect maintenance," European Journal of Operational Research, vol. 94, pp. 425-438, 1996.

[70] S. Taghipour and D. Banjevic, "Optimal inspection of a complex system subject to periodic and opportunistic inspections and preventive replacements," European Journal of Operational Research, vol. 220, pp. 649-660, 2012.

[71] V. Babishin and S. Taghipour, "Maintenance and Inspection Optimization of a System with Hidden Failures," Proceedings of the IIE Annual Conference 2015, pp. 1553-1562, 2015.

[72] H. Wang and H. Pham, Reliability and Optimal Maintenance, London: Springer, 2006.

[73] R. Dekker, R. E. Wildeman and F. A. van der Duyn Schouten, "A review of multicomponent maintenance models with economic dependence," Mathematical Methods of Operations Research, vol. 45, pp. 411-435, 1997.

[74] J. Shao and L. R. Lamberson, "Modeling a Shared-Load k-out-of-n: G System," IEEE Transactions on Reliability, vol. 40, no. 2, pp. 205-209, 1991.

[75] S. Taghipour, D. Banjevic and A. K. S. Jardine, "Reliability Analysis of Maintenance Data for Complex Medical Devices," Quality and Reliability Engineering International, vol. 27, no. 1, p. 71-84, 2011.

[76] P. A. Scarf, "On the Application of Mathematical Models in Maintenance," European Journal of Operational Research, vol. 99, pp. 493-506, 1997.

[77] W. Wang and A. H. Christer, "Solution algorithms for a nonhomogeneous multicomponent inspection model," Computers \& Operations Research, vol. 30, pp. 19-34, 2003. 
[78] V. Zille, C. Bérenguer, A. Grall and A. Despujols, "Modelling multicomponent systems to quantify reliability centred maintenance strategies," Proc. IMechE Part O: J. Risk and Reliability, vol. 225, pp. 141-160, 2011.

[79] J. C. Hartman and A. Murphy, "Finite-horizon equipment replacement analysis," IIE Transactions, vol. 38, pp. 409-419, 2006.

[80] W. Hyman, "The Theory and Practice of Preventive Maintenance," Journal of Clinical Engineering, vol. 28, no. 1, pp. 31-36, 2003.

[81] J. H. Seo and D. S. Bai, "An Optimal Maintenance Policy for a System under Periodic Overhaul," Mathematical and Computer Modelling, vol. 39, pp. 373-380, 2004.

[82] C.-C. Chang, "Optimum preventive maintenance policies for systems subject to random working times, replacement, and minimal repair," Computers \& Industrial Engineering, vol. 67, pp. 185-194, 2014.

[83] T. Nakagawa and S. Mizutani, "A Summary of maintenance policies for a finite interval," Reliability Engineering and System Safety, vol. 94, pp. 89-96, 2009.

[84] C. Sriram and A. Haghani, "An optimization model for aircraft maintenance scheduling and re-assignment," Transportation Research Part A, vol. 37, pp. 29-48, 2003.

[85] S. Taghipour, D. Banjevic and A. K. S. Jardine, "An Inspection Optimization Model for a System subject to Hidden Failures," in Proceedings of the 2010 ICOMS Asset Management Conference, 2010.

[86] V. Babishin and S. Taghipour, "Joint Maintenance and Inspection Optimization of a kout-of-n System," Proceedings of the Annual Reliability and Maintainability Symposium 2016, pp. 523-528, 2016.

[87] V. Babishin and S. Taghipour, "Joint Optimal Maintenance and Inspection for a k-outof-n System," Int. J. Adv. Manuf. Technol., vol. 87, no. 5, pp. 1739-1749, 2016.

[88] I. Gertsbakh, Reliability theory with applications to preventive maintenance, New York: Springer, 2000.

[89] I. Norros, P. Kuusela, J. Lapuyade-Lahorgue, M. Naldi and M. Sirviö, "Repairable Systems With Dependent Components: Stochastic Process Techniques and Models," IEEE Transactions on Reliability, vol. 63, no. 4, pp. 899-912, 2014.

[90] V. V. Anisimov and Ü. Gürler, "An Approximate Analytical Method of Analysis of a Threshold Maintenance Policy for a Multiphase Multicomponent Model," Cybernetics and Systems Analysis, vol. 39, no. 3, pp. 325-337, 2003.

[91] T. Sun, Q. Zhao and P. B. Luh, "Optimization of Joint Replacement Policies for Multipart Systems by a Rollout Framework," IEEE Transactions on Automation Science and Engineering, vol. 5, no. 4, pp. 609-619, 2008.

[92] G. Tu, P. B. Luh and Q. Zhao, "An optimization method for joint replacement decisions in maintenance," 43rd IEEE Conference on Decision and Control, vol. 4, pp. 3674-3679, 2004.

[93] H. Boudhar, M. Dahane and N. Rezg, "A new inspection heuristic for a condition-based maintenance policy in a spare part returns context," IEEE Transactions of International Conference on Systems, Man and Cybernetics, pp. 1300-1305, 2013. 
[94] D. Zhou, Z. Yu, H. Zhang and S. Weng, "A novel grey prognostic model based on Markov process and grey incidence analysis for energy conversion equipment degradation," Energy, vol. 109, pp. 420-429, 2016.

[95] Q.-S. Jia, "A Structural Property of Optimal Policies for Multi-Component Maintenance Problems," IEEE Transactions on Automation Science and Engineering, vol. 7, no. 3, pp. 677-680, 2010.

[96] S.-Z. Yu, "Hidden semi-Markov models," Artificial Intelligence, vol. 174, pp. 215-243, 2010.

[97] D. N. P. Murthy and D. G. Nguyen, "Optimal age-policy with imperfect preventive maintenance," IEEE Transactions on Reliability, vol. 30, pp. 80-81, 1981.

[98] M. Brown and F. Proschan, "Imperfect repair," Journal of Applied Probability, vol. 20, p. 851-859, 1983.

[99] T. Nakagawa and K. Yasui, "Optimal policies for a system with imperfect maintenance," IEEE Transactions on Reliability, vol. 36, no. 5, pp. 631-633, 1987.

[100] C.-C. Chang, S.-H. Sheu, Y.-L. Chen and Z. G. Zhang, "A multi-criteria optimal replacement policy for a system subject to shocks," Computers \& Industrial Engineering, vol. 61, pp. 1035-1043, 2011.

[101] X. Zhang and J. Zeng, "Deterioration state space partitioning method for opportunistic maintenance modelling of identical multi-unit systems," International Journal of Production Research, vol. 53, no. 7, pp. 2100-2118, 2015.

[102] Y. Lam, "A Geometric Process d-Shock Maintenance Model," IEEE Transactions on Reliability, vol. 58, no. 2, pp. 389-396, 2009.

[103] X. Zhang and J. Zeng, "A general modeling method for opportunistic maintenance modeling of multi-unit systems," Reliability Engineering and System Safety, vol. 140, pp. 176-190, 2015.

[104] L. Bian and N. Gebraeel, "Stochastic modeling and real-time prognostics for multicomponent systems with degradation rate interactions," IIE Transactions, vol. 46, no. 5, pp. 470-482, 2014.

[105] H. C. Vu, P. Do, A. Barros and C. Bérenguer, "Maintenance planning and dynamic grouping for multi-component systems with positive and negative economic dependencies," IMA Journal of Management Mathematics, vol. 26, pp. 145-170, 2015.

[106] S. Taghipour and D. Banjevic, "Inspection frequency," Industrial Engineer, vol. 44, no. $10,2012$.

[107] S.-H. Sheu, "A generalized model for determining optimal number of minimal repairs before replacement," European Journal of Operational Research, vol. 69, pp. 38-49, 1993.

[108] S.-H. Sheu and W. S. Griffith, "Optimal Number of Minimal Repairs before Replacement of a System Subject to Shocks," Naval Research Logistics, vol. 43, pp. 319333, 1996.

[109] S.-H. Sheu, H.-N. Tsai, T.-S. Hsu and F.-K. Wang, "Optimal number of minimal repairs before replacement of a deteriorating system with inspections," International Journal of Systems Science, vol. 46, no. 8, pp. 1367-1379, 2015. 
[110] S. Taghipour, Reliability and Maintenance of Medical Devices, LAP Lambert Academic Publishing, 2012.

[111] S. Taghipour, D. Banjevic and A. K. S. Jardine, "Risk-Based Inspection and Maintenance for Medical Equipment," Proceedings of the International Industrial Engineering Research Conference, pp. 104-109, 2008.

[112] L. Cui, M. Xie and H. Loh, "Inspection schemes for general systems," IIE Transactions, vol. 36, pp. 817-825, 2004.

[113] P. J. Boland, "Periodic replacement when minimal repair costs vary with time," Naval Research Logistics Quarterly, vol. 29, pp. 541-546, 1982.

[114] R. Ahmad and S. Kamaruddin, "An overview of time-based and condition-based maintenance in industrial application," Computers \& Industrial Engineering, vol. 63, p. 135-149, 2012.

[115] S.-H. Sheu, T.-H. Liu, Z.-G. Zhang and T.-M. Chang, "Optimal number of repairs before replacement for a two-unit system subject to non-homogeneous pure birth process," Computers \& Industrial Engineering, vol. 69, p. 71-76, 2014.

[116] C.-C. Chang, S.-H. Sheu and Y.-L. Chen, "Optimal number of minimal repairs before replacement based on a cumulative repair-cost limit policy," Computers \& Industrial Engineering, vol. 59, p. 603-610, 2010.

[117] K. T. Huynh, A. Barros, C. Bérenguer and I. T. Castro, "A periodic inspection and replacement policy for systems subject to competing failure modes due to degradation and traumatic events," Reliability Engineering and System Safety, vol. 96, p. 497-508, 2011.

[118] E. A. Gunn and C. Diallo, "Optimal opportunistic indirect grouping of preventive replacements in multicomponent systems," Computers \& Industrial Engineering, vol. 90, pp. 281-291, 2015.

[119] K. S. Moghaddam and J. S. Usher, "Sensitivity analysis and comparison of algorithms in preventive maintenance and replacement scheduling optimization models," Computers \& Industrial Engineering, vol. 61, pp. 64-75, 2011.

[120] A. Alrabghi and A. Tiwari, "State of the art in simulation-based optimisation for maintenance systems," Computers \& Industrial Engineering, vol. 82, pp. 167-182, 2015.

[121] M. Moura, L. D. Lins, E. L. D. Soares and R. Pascual, "A Multi-Objective Genetic algorithms for determining efficient Risk-Based Inspection programs," Reliability Engineering and System Safety, vol. 133, pp. 253-265, 2015.

[122] M. Gen and R. Cheng, Genetic Algorithms and Engineering Optimization, New York: Wiley, 1999.

[123] D. E. Goldberg, Genetic algorithms in search, optimization, and machine learning, Reading: Addison-Wesley, 1989.

[124] A. Grall, L. Dieulle, C. Bérenguer and M. Roussignol, "Continuous-Time PredictiveMaintenance Scheduling for a Deteriorating System," IEEE Transactions on Reliability, vol. 51, no. 2, pp. 141-150, 2002.

[125] Y. Hajipour and S. Taghipour, "Non-Periodic Inspection Optimization of MultiComponent and k-out-of-n Systems Using Genetic Algorithm," Reliability Engineering and System Safety, 2016. 
[126] V. Babishin and S. Taghipour, "Optimal Maintenance Policy for Multicomponent Systems with Periodic and Opportunistic Inspections and Preventive Replacements," Applied Mathematical Modelling, vol. 40, no. 23-24, pp. 10480 - 10505, 2016.

[127] M. K. Salameh and M. Y. Jaber, "Optimal lot sizing with regular maintenance interruptions," Applied Mathematical Modelling, vol. 21, no. 2, pp. 85-90, 1997.

[128] M. Y. Jaber, Ed., Learning Curves: Theory, Models, and Applications, Boca Raton, FL: CRC Press-Taylor \& Francis Group, 2011.

[129] R. Schnittker, M. Schmettow, F. Verhoeven and J. Schraagen, "Combining situated Cognitive Engineering with a novel testing method in a case study comparing two infusion pump interfaces," Applied Ergonomics, vol. 55, pp. 16-26, 2016.

[130] R. Fidler and M. Johnson, "Human Factors Approach to Comparative Usability of Hospital Manual Defibrillators," Resuscitation, vol. 101, pp. 71-76, 2016.

[131] R. Fairbanks, S. Caplan, P. Bishop, A. Marks and M. Shah, "Usability Study of Two Common Defibrillators Reveals Hazards," Annals of Emergency Medicine, vol. 50, no. 4, pp. 424-432, 2007.

[132] R. Fairbanks and S. Caplan, "Poor interface design and lack of usability testing facilitate medical error," Joint Commission Journal on Quality and Safety, vol. 30, no. 10, pp. 579-584, 2004.

[133] O. Krichevsky and G. Bonnet, "Fluorescence correlation spectroscopy: The technique and its applications," Report on Progress in Physics, vol. 65, p. 251-297, 2002.

[134] W. Gerstner and W. Kistler, Spiking Neuron Models: Single Neurons, Populations, Plasticity, Cambridge Univ. Press, 2002.

[135] J. D. Scargle, "Studies in astronomical time series analysis. V. Bayesian blocks, a new method to analyze structure in photon counting data," Astrophys. J., vol. 504, pp. 405418, 1998.

[136] T. Zhang and S. C. Kou, "Nonparametric Inference of Doubly Stochastic Poisson Process Data via the Kernel Method," The Annals of Applied Statistics, vol. 4, no. 4, pp. 19131941, 2010.

[137] C. P. Neuman and N. M. Bonhomme, "Optimal Inspection and Repair Schedules for Multicomponent Systems," IEEE Transactions on Systems, Man and Cybernetics, pp. 58-65, 1974.

[138] Y. Xiang, C. R. Cassady and E. A. Pohl, "Optimal maintenance policies for systems subject to a Markovian operating environment," Computers \& Industrial Engineering, vol. 62, pp. 190-197, 2012. 